\title{
Biomass Feedstock Supply System Design and Analysis
}

Jacob J. Jacobson, Mohammad S. Roni, Patrick Lamers, Kara G. Cafferty

September 2014

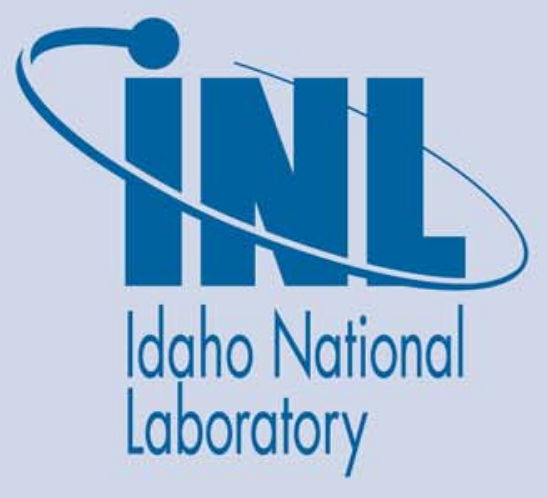

The INL is a U.S. Department of Energy National Laboratory operated by Battelle Energy Alliance 
INL/EXT-14-32377

\title{
Biomass Feedstock Supply System Design and Analysis
}

Jacob J. Jacobson, Mohammad S. Roni, Patrick Lamers, Kara G. Cafferty

September 2014

\author{
Idaho National Laboratory \\ Idaho Falls, Idaho 83415
}

http://www.inl.gov

Prepared for the

U.S. Department of Energy

Office of Biomass Program

Under DOE Idaho Operations Office

Contract DE-AC07-05ID14517 


\section{DISCLAIMER}

This information was prepared as an account of work sponsored by an agency of the U.S. Government. Neither the U.S. Government nor any agency thereof, nor any of their employees, makes any warranty, expressed or implied, or assumes any legal liability or responsibility for the accuracy, completeness, or usefulness, of any information, apparatus, product, or process disclosed, or represents that its use would not infringe privately owned rights. References herein to any specific commercial product, process, or service by trade name, trademark, manufacturer, or otherwise, does not necessarily constitute or imply its endorsement, recommendation, or favoring by the U.S. Government or any agency thereof. The views and opinions of authors expressed herein do not necessarily state or reflect those of the U.S. Government or any agency thereof. 


\title{
Biomass Feedstock Supply System Design and Analysis
}

\author{
September 2014
}

Idaho National Laboratory

Bioenergy Program

Idaho Falls, Idaho 83415

http://www.inl.gov

Prepared for the U.S. Department of Energy Office of Biomass Program Under DOE Idaho Operations Office Contract DE-AC07-05ID14517 


\section{Executive Summary}

The U.S. Department of Energy (DOE) aims to make cellulosic biofuels competitive with petroleum-based fuels at a modeled cost of mature bio-oil technology of $\$ 3 /$ gallon gasoline equivalent (gge) (\$2011) by the year 2022. The DOE Bioenergy Technologies Office (BETO) Terrestrial Feedstock Technology Area supports this goal by demonstrating a modeled delivered feedstock cost of $\$ 80 /$ dry T (dry U.S. short ton) by the year 2017. The purpose of this report is to document a feasible feedstock supply system for various conversion pathway designs capable of achieving this 2017 target. This design is referred to in this report as the "2017 Design Case." Idaho National Laboratory (INL) supports the U.S. Department of Energy's bioenergy research program. As part of the research program INL investigates the feedstock logistics economics and sustainability of these fuels. A series of reports were published between 2000 and 2013 to demonstrate the feedstock logistics cost. Those reports were tailored to specific feedstock and conversion process. Although those reports are different in terms of conversion, the strategies to improve logistics operations are similar at each conversion process. Therefore, this report is designed in such a way that it can capture different feedstock logistics cost while eliminating the need of writing a conversion specific design report.

This report provides feedstock design cost analysis for five conversion pathways: 1) Biological Conversion of Sugars to Hydrocarbons, 2) Lignocellulosic Biomass conversion to Hydrocarbon Fuels via Fast Pyrolysis and Hydrotreating Bio-Oil Pathway, 3) Catalytic Conversion of Sugars to Hydrocarbons ,4) Conversion of Lignocellulosic Biomass to Hydrocarbon Fuels via Thermochemical Pathways with In Situ and Ex Situ Upgrading of Fast Pyrolysis Vapors, and 5) Conversion of Lignocellulosic Biomass to High Octane Gasoline via Indirect Gasification and Methanol Intermediate. The delivered feedstock composition assumed at the process design for Biological Conversion of Sugars to Hydrocarbons and Catalytic Conversion of Sugars to Hydrocarbons is the same. Therefore, feedstock supply chain design cost analysis will be the same for these two conversions pathways. Similarly the delivered feedstock composition assumed at the process design for conversion of Lignocellulosic Biomass to Hydrocarbon Fuels via 1) Fast Pyrolysis and Hydrotreating Bio-Oil Pathway, 2) Thermochemical Pathways with In Situ and Ex Situ Upgrading of Fast Pyrolysis Vapors, and 3) Indirect Gasification and Methanol Intermediate is the same. As a result feedstock design cost analysis is the same for these three conversion pathways. As each of these conversion pathways mature and additional information is added to the feedstock in-feed specifications the chapters for each pathway will be updated based on the new information.

The goal of the 2017 Design Case is to enable expansion of biofuels production beyond highly productive resource areas by breaking the reliance of cost-competitive biofuel production on a single, abundant, low-cost feedstock. If this goal is not achieved, biofuel plants are destined to be small and/or clustered in select regions of the country that have a lock on low-cost feedstock. To put the 2017 cost target into perspective of past accomplishments of the cellulosic ethanol pathway, the $\$ 80 /$ dry ton target encompasses total delivered feedstock cost, including both grower payment and logistics costs, while meeting all conversion in-feed quality targets. The 2012 programmatic target of $\$ 35 /$ dry ton included only logistics costs with a limited focus on biomass quality. 
The 2017 Design Case explores two approaches to addressing the logistics challenge: one is an agronomic solution based on blending and integrated landscape management and the second is a logistics solution based on distributed biomass preprocessing depots. The concept behind blended feedstocks and integrated landscape management is to gain access to more regional feedstock at lower access fees (i.e., grower payment) and to reduce preprocessing costs by blending high quality feedstocks with marginal quality feedstocks. Blending has been used in the grain industry for a long time; however, the concept of blended feedstocks in the biofuel industry is a relatively new concept. The blended feedstock strategy relies on the availability of multiple feedstock sources that are blended using a least-cost formulation within an economical supply radius, which, in turn, decreases the grower payment by reducing the amount of any single biomass. This report will introduce the concepts of blending and integrated landscape management and justify their importance in meeting the 2017 programmatic goals.

The biomass feedstock supply system is a combination of multiple operations that include harvest and collection, storage, preprocessing, and transportation. Each operation within the supply system incurs a cost while influencing the biomass quality. This report summarizes the improvements that are being targeted, based on the research objectives in the following five research areas: (1) blending, (2) harvest and collection, (3) storage, (4) preprocessing, and (5) transportation and handling. Feedstock logistics research aims to reduce delivered cost, improve or preserve feedstock quality, and expand feedstock access. Strategies to improve logistics operations include (1) organizing logistics in innovative ways, (2) improving existing operations for efficiency and interaction with other operations, and (3) implementing new technologies to overcome quality issues. The result is a new advanced biomass supply system that meets the $\$ 80 /$ dry T. delivered cost. 
Table E-1. Summary of assumptions underpinning progressive design implementations for biological conversion of sugars to hydrocarbons and catalytic conversion of sugars to hydrocarbons.

\begin{tabular}{|c|c|c|c|}
\hline & 2012 Conventional Design & Baseline & 2017 Design Case \\
\hline Feedstock(s) & Corn stover & Corn stover & $\begin{array}{l}\text { Blended feedstock: corn } \\
\text { stover, switchgrass, and } \\
\text { select municipal solid waste } \\
\text { (MSW) }\end{array}$ \\
\hline Grower payment & Minimal & $\begin{array}{l}\text { Increases based on marginal } \\
\text { cost differential }\end{array}$ & $\begin{array}{l}\text { Calculated and modeled } \\
\text { according to specific } \\
\text { location and resource } \\
\text { blend/formulation }\end{array}$ \\
\hline Moisture & Field dried to $12 \%$ & $\begin{array}{l}\text { Arrives at } 30 \% \\
\text { Dried to } 20 \%\end{array}$ & $\begin{array}{l}\text { Arrives: corn stover } 30 \% \text {, } \\
\text { switchgrass } 20 \% \text {, and } \\
\text { MSW } 20 \% \text {; } \\
\text { All dried to } 9 \%\end{array}$ \\
\hline Ash & $\begin{array}{l}\text { No ash management } \\
\text { assumed }\end{array}$ & $\begin{array}{l}11 \% \text {, dockage accessed for } \\
\text { ash content Greater than } 5 \% \\
\text { spec }\end{array}$ & $\begin{array}{l}\text { Blended ash content of } 4.9 \% \\
\text { Corn stover: multi-pass } 7 \% \text {; } \\
\text { single-pass } 3.5 \% \\
\text { Switchgrass: } 4 \% \\
\text { MSW: } 10 \%\end{array}$ \\
\hline Logistics & Uses existing systems & Uses existing systems & $\begin{array}{l}\text { Fractional milling } \\
\text { High-moisture densification } \\
\text { Rail transportation for MSW }\end{array}$ \\
\hline $\begin{array}{l}\text { Quality controls } \\
\text { (passive) }\end{array}$ & $\begin{array}{l}\text { Field drying to meet } \\
\text { moisture spec } \\
\text { Ample available resource; } \\
\text { quality spec manually } \\
\text { selected }\end{array}$ & $\begin{array}{l}\text { Dockage fee assessed to } \\
\text { supplier for below-quality } \\
\text { material }\end{array}$ & $\begin{array}{l}\text { Multi versus single-pass } \\
\text { harvest/ collection } \\
\text { Harvest/collection and } \\
\text { storage best management } \\
\text { practices }\end{array}$ \\
\hline $\begin{array}{l}\text { Quality controls } \\
\text { (active) }\end{array}$ & None assumed & Rotary drying & $\begin{array}{l}\text { Multiple resource } \\
\text { blending/formulation } \\
\text { High-moisture densification } \\
\text { High-efficiency pellet drying }\end{array}$ \\
\hline $\begin{array}{l}\text { Meets quality } \\
\text { target }\end{array}$ & No & Yes & Yes \\
\hline Meets cost target & Yes & No & Yes \\
\hline $\begin{array}{l}\text { Accesses dispersed } \\
\text { resources }\end{array}$ & No & No & Yes \\
\hline
\end{tabular}


Table E-2. Summary of assumptions underpinning progressive design implementations for thermochemical conversion(Fast Pyrolysis and Hydro treating Bio-Oil Pathway, Thermochemical Pathways with In Situ and Ex Situ Upgrading of Fast Pyrolysis Vapors, Indirect Gasification) (INL 2017 Design Case).

\begin{tabular}{|c|c|c|c|}
\hline & 2012 Conventional Design & Baseline & 2017 Design Case \\
\hline Feedstock(s) & Pulpwood & Pulpwood & $\begin{array}{l}\text { Blended feedstock: } \\
\text { pulpwood, wood residues, } \\
\text { switchgrass, and select } \\
\text { construction and demolition } \\
\text { wastes (C \&D) }\end{array}$ \\
\hline Grower payment & $\begin{array}{l}\text { Breakeven cost of } \\
\text { production }\end{array}$ & $\begin{array}{l}\text { Increases based on marginal } \\
\text { cost differential }\end{array}$ & $\begin{array}{l}\text { Calculated and modeled } \\
\text { according to specific location } \\
\text { and resource } \\
\text { blend/formulation }\end{array}$ \\
\hline Moisture & Field dried to $40 \%$ & Field dried to $40 \%$ & $\begin{array}{l}\text { Arrives: Pulpwood chips } \\
30 \% \text { wood residue chips } \\
30 \% \text {, switchgrass } 20 \% \text {, and } \\
\text { C\& D ground } 20 \% \text {; } \\
\text { All dried to } 9 \% \text { pellets }\end{array}$ \\
\hline Ash & Debark/delimb & Debark/delimb & $\begin{array}{l}\text { Debark/delimb pulpwood } \\
\text { Trommel screen residues } \\
\text { Wash and sort C\& D waste } \\
\text { Blended ash content of }<1 \% \\
\text { Debarked pulpwood }<1 \% \text {, } \\
\text { screened wood residues } \\
1.4 \% \text {; washed and sorted } \\
\text { C\&D } 1.0 \%\end{array}$ \\
\hline Logistics & Uses existing systems & Uses existing systems & $\begin{array}{l}\text { Pneumatics attached to } \\
\text { hammermill } \\
\text { High-moisture densification }\end{array}$ \\
\hline $\begin{array}{l}\text { Quality controls } \\
\text { (passive) }\end{array}$ & $\begin{array}{l}\text { Field drying to reduce } \\
\text { moisture } \\
\text { Ample available resource; } \\
\text { quality spec manually } \\
\text { selected }\end{array}$ & $\begin{array}{l}\text { Field drying to meet } \\
\text { moisture spec }\end{array}$ & $\begin{array}{l}\text { Harvest/collection and } \\
\text { storage best management } \\
\text { practices for pulpwood and } \\
\text { switchgrass } \\
\text { More rigorous field drying of } \\
\text { pulpwood and residues }\end{array}$ \\
\hline $\begin{array}{l}\text { Quality controls } \\
\text { (active) }\end{array}$ & Waste heat dryer & Rotary drying & $\begin{array}{l}\text { Multiple resource } \\
\text { blending/formulation } \\
\text { High-moisture densification } \\
\text { High-efficiency pellet drying }\end{array}$ \\
\hline $\begin{array}{l}\text { Meets quality } \\
\text { target }\end{array}$ & Yes & Yes & Yes \\
\hline Meets cost target & Yes & No & Yes \\
\hline $\begin{array}{l}\text { Accesses dispersed } \\
\text { resources }\end{array}$ & No & No & Yes \\
\hline
\end{tabular}




\section{Authors and Contributors}

Jacob J. Jacobson

Mohammad S. Roni

Kara G. Cafferty

Kevin Kenney

Erin Searcy

Jason Hansen 


\section{Table of Contents}

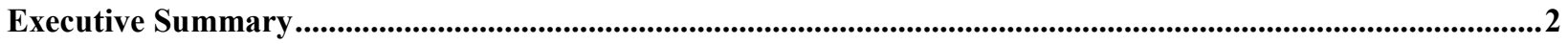

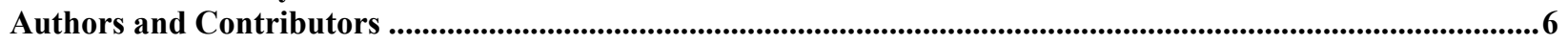

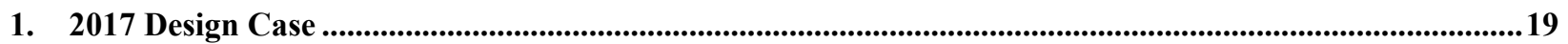

2. Limitations of Conventional Supply System Designs ...............................................................................................21

2.1 Expansion Beyond Highly Productive Regions .................................................................... 22

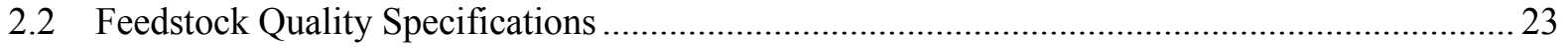

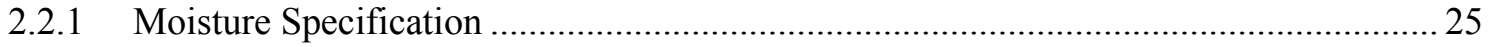

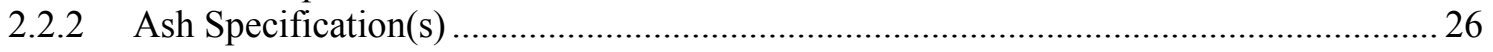

3. Moving beyond the 2012 Conventional Design ...............................................................................................29

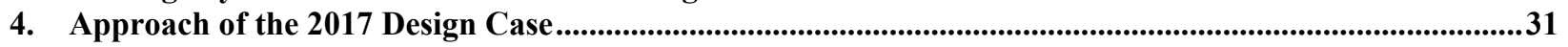

4.1 Addressing the Farm Gate Price Challenge …........................................................................ 31

4.2 Addressing the Feedstock Specification Challenge ….............................................................. 33

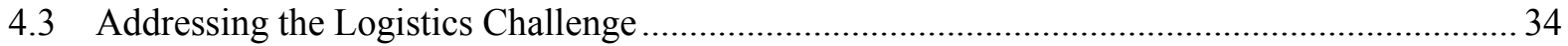

5. 2017 Feedstock Supply System Design .....................................................................................................36

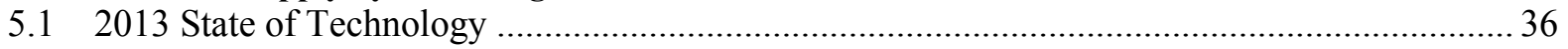

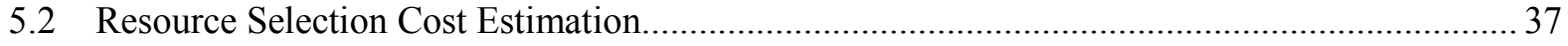

6. Feedstock Supply System Unit Operations ……....................................................................................................39

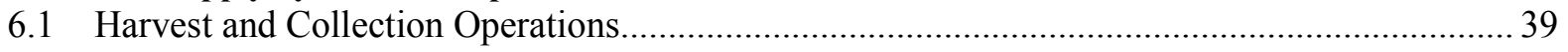

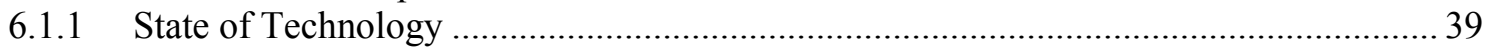

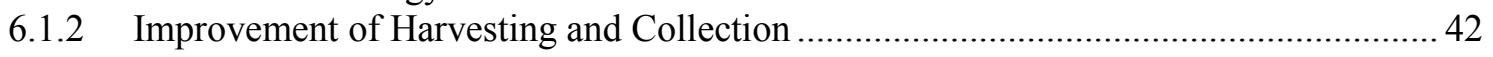

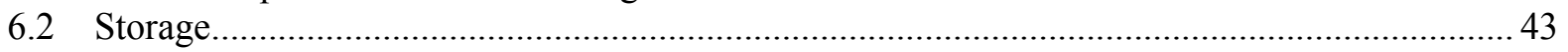

6.2.1 State of Technology Herbaceous Residues/Energy Crops .......................................... 43

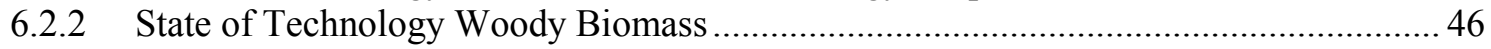

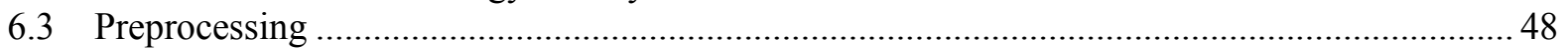

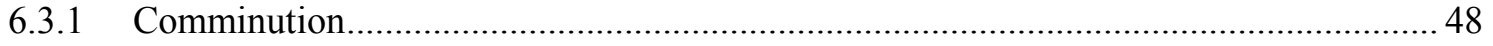

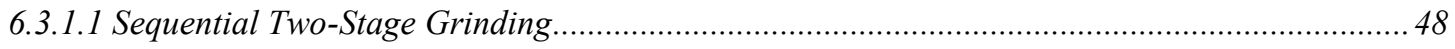

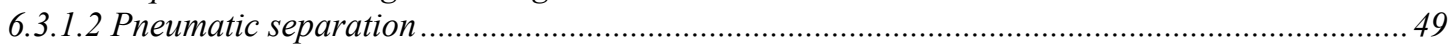

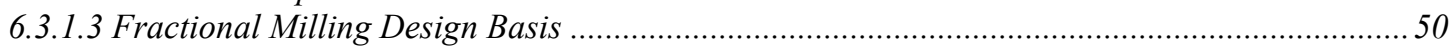

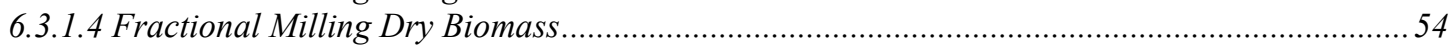

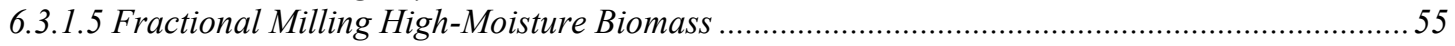

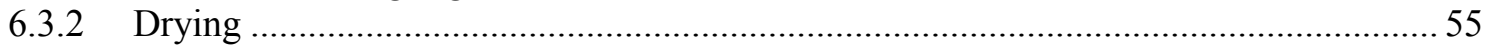

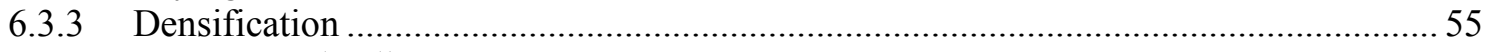

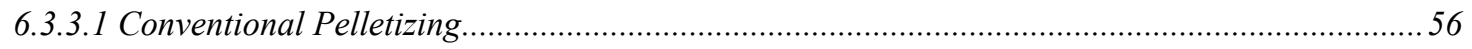

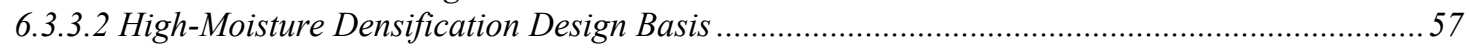

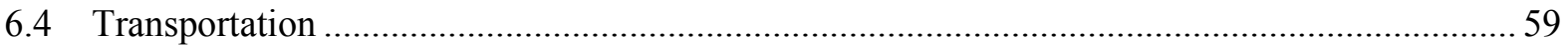

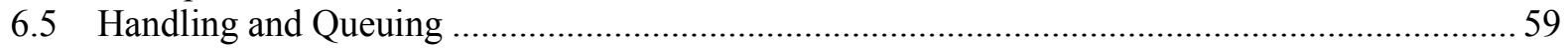

7. Supply System Design: Arrangement of Unit Operations ................................................................................60

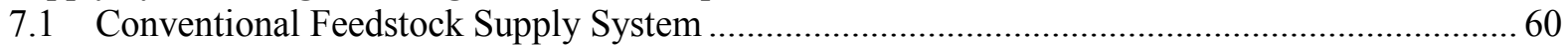

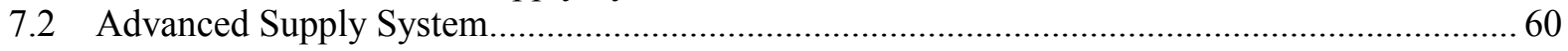

8. Biological Conversion of Sugars to Hydrocarbons .................................................................................................62

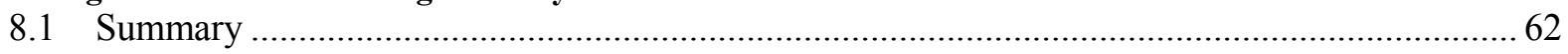

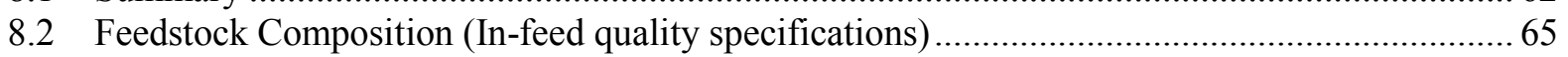

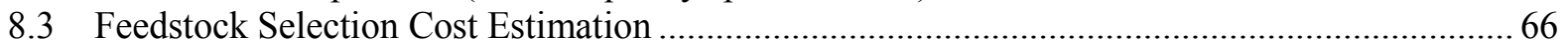

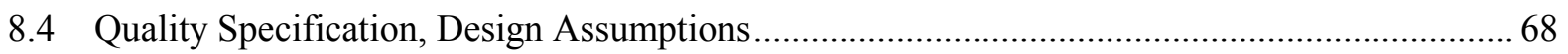

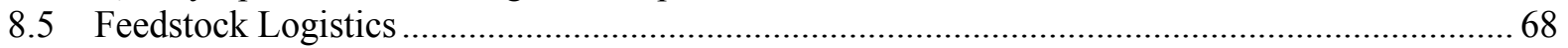

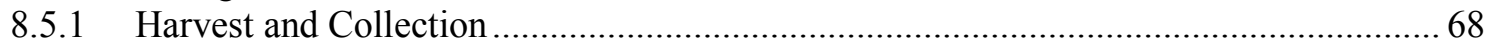

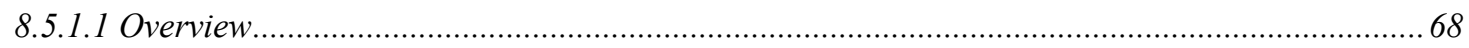

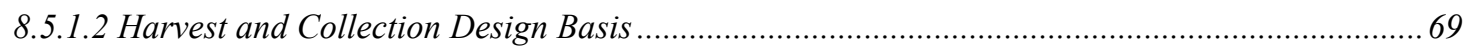




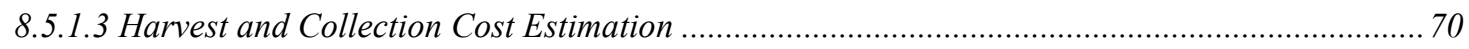

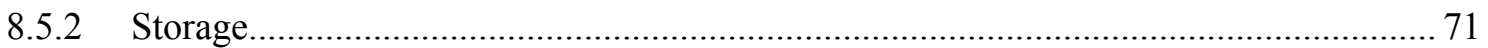

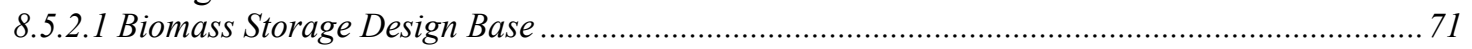

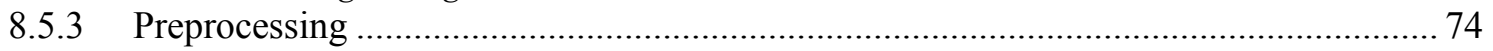

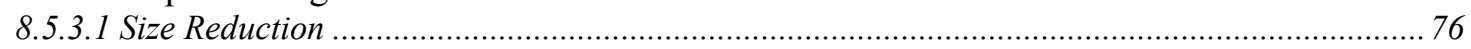

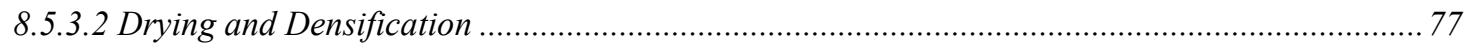

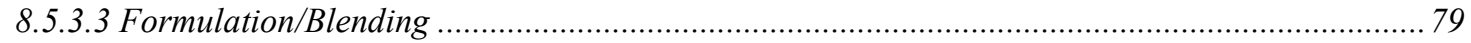

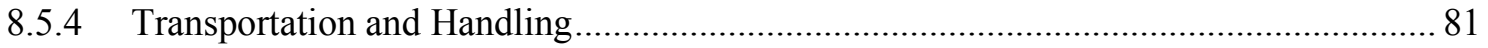

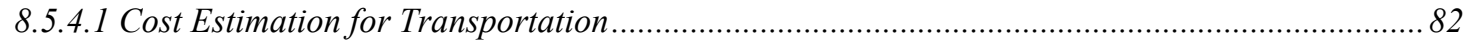

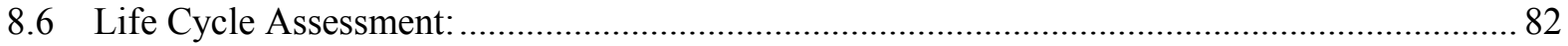

9. Conversion of Lignocellulosic Biomass to Hydrocarbon Fuels: Fast Pyrolysis and Hydrotreating Bio-Oil

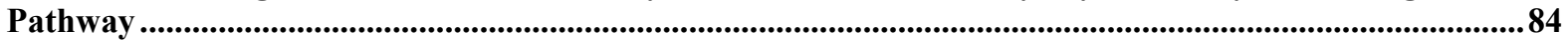

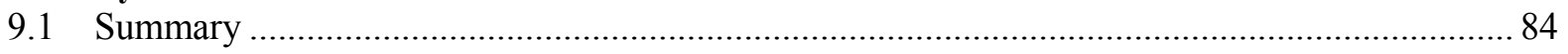

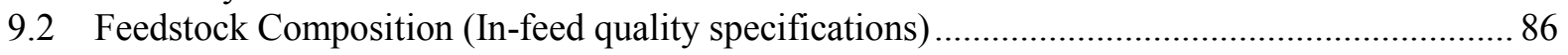

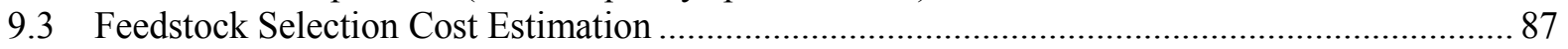

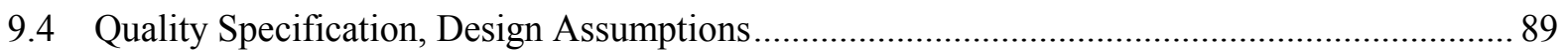

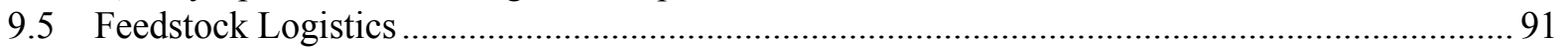

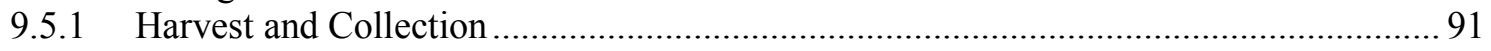

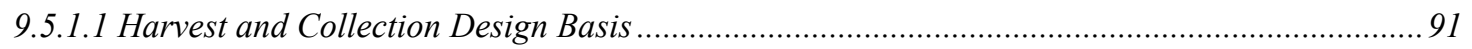

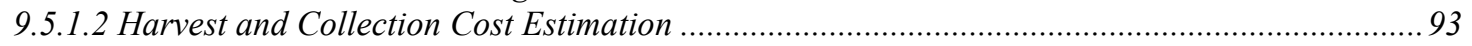

9.5.2 Storage

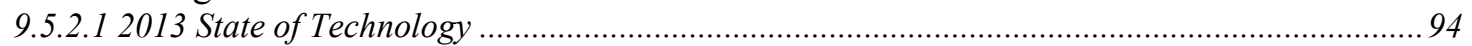

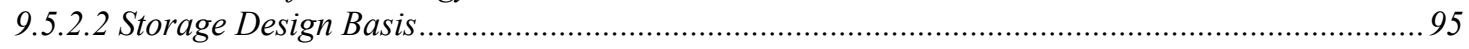

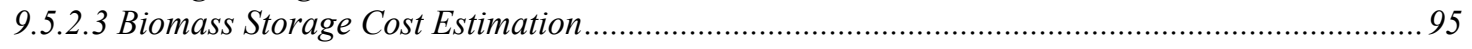

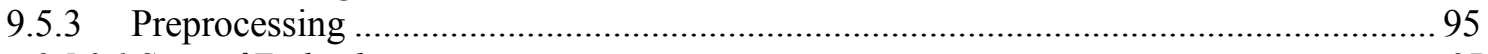

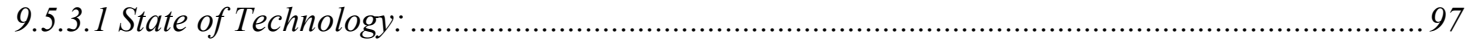

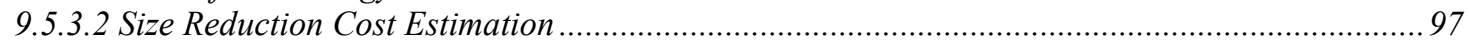

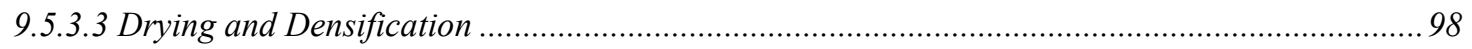

9.5.3.4 Cost Estimation for High-Moisture Densification .................................................................. 99

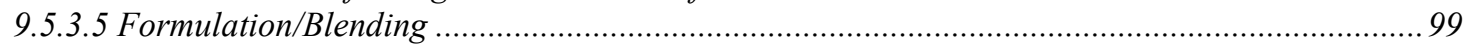

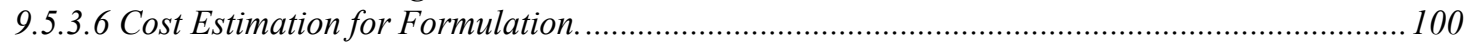

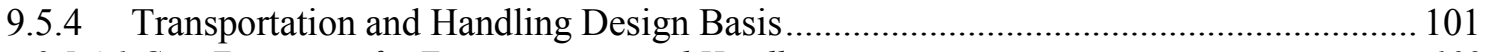

9.5.4.1 Cost Estimation for Transportation and Handling .............................................................. 102

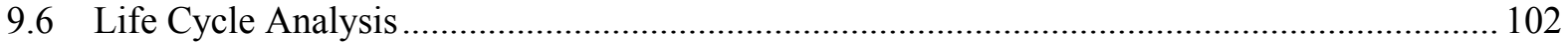

10. Dilute-Acid and Enzymatic Deconstruction of Biomass to Sugars and Catalytic Conversion of Sugars to

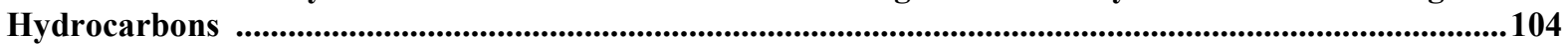

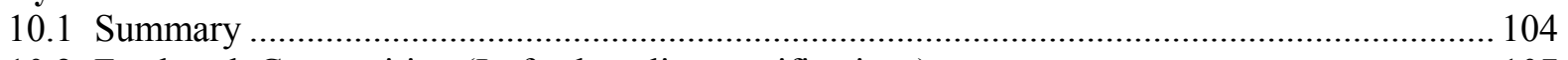

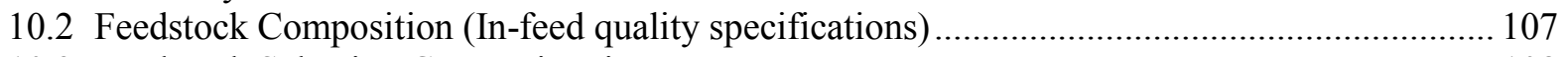

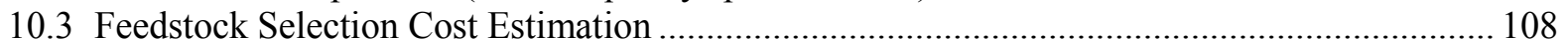

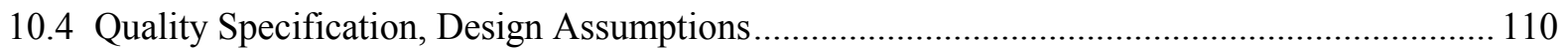

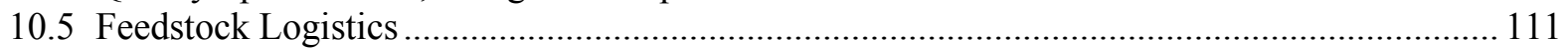

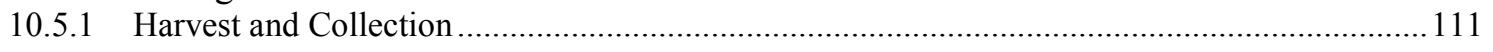

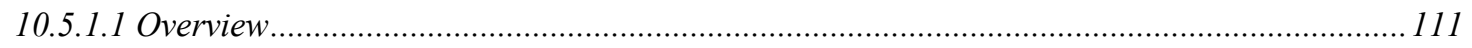

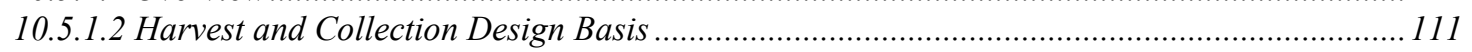

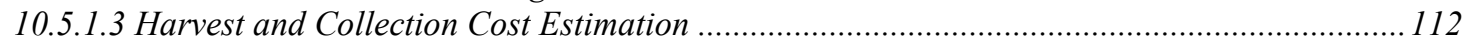

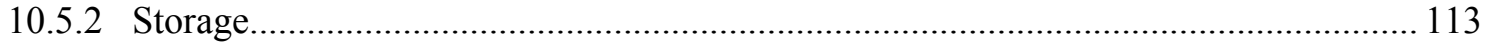

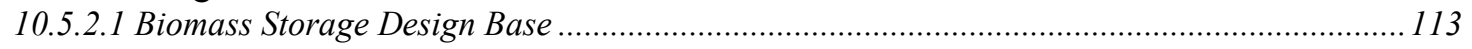

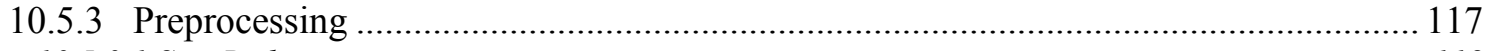

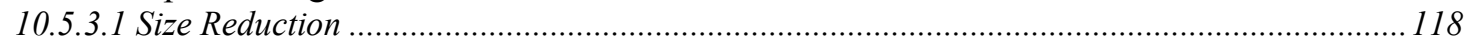

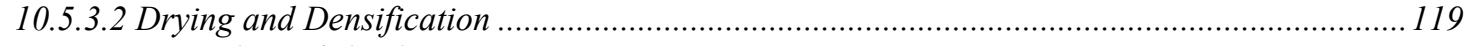

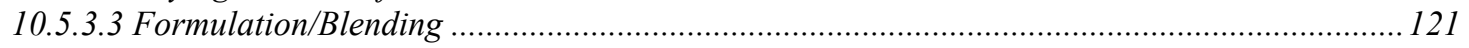

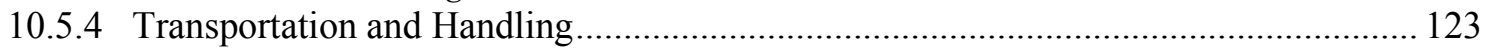




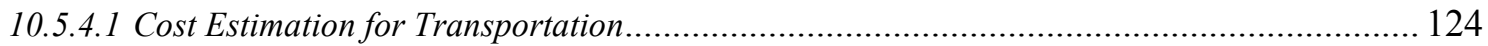

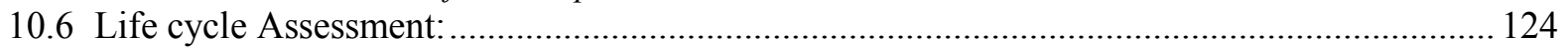

11. Conversion of Lignocellulosic Biomass to Hydrocarbon Fuels: Thermochemical Pathways with In Situ

and Ex Situ Upgrading of Fast Pyrolysis Vapors .....................................................................................126

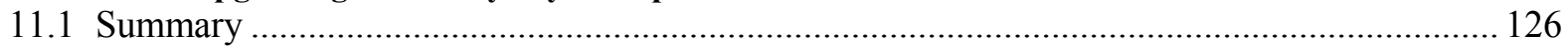

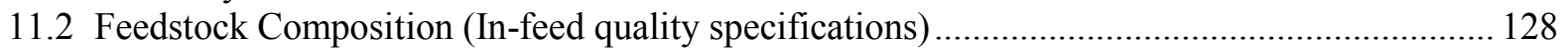

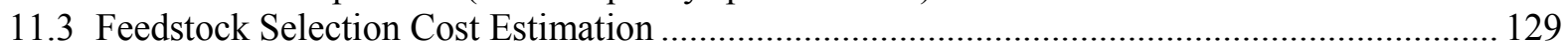

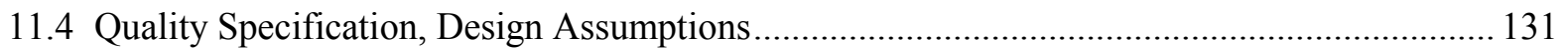

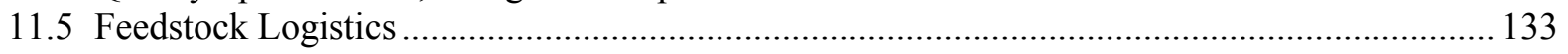

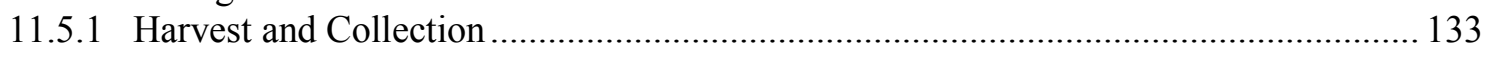

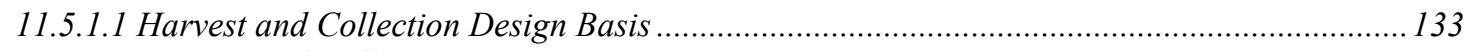

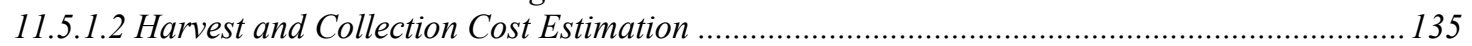

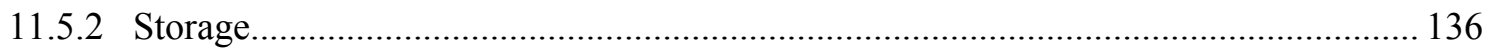

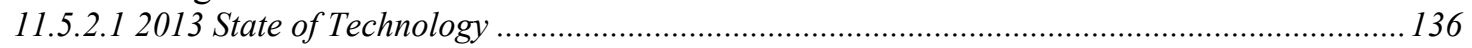

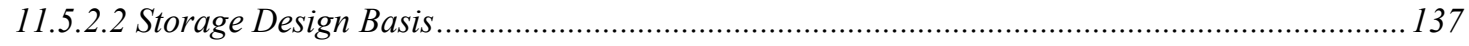

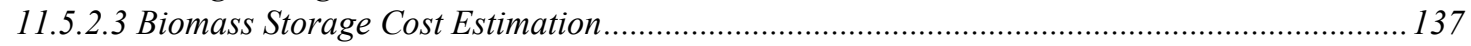

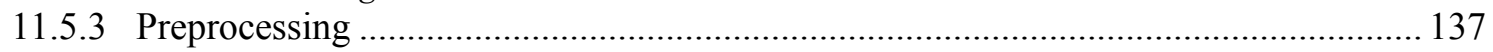

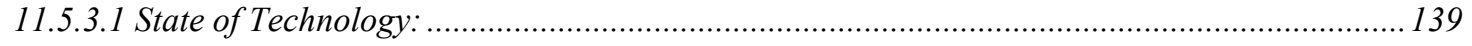

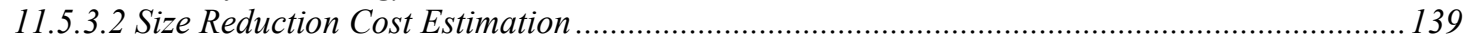

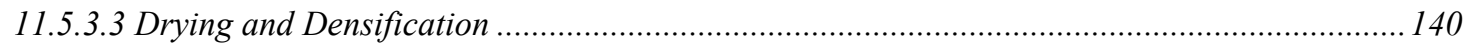

11.5.3.4 Cost Estimation for High-Moisture Densification ................................................................ 141

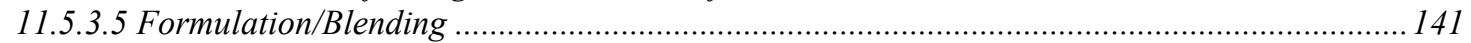

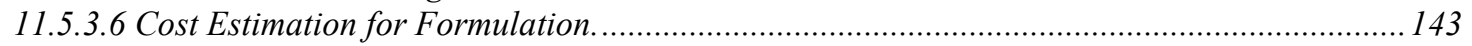

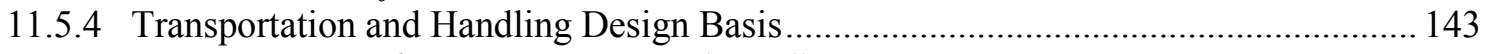

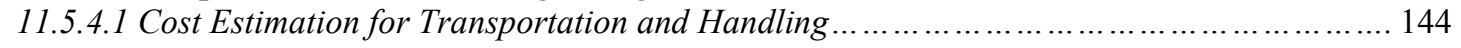

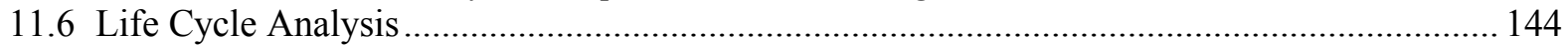

12. Dilute-Acid Conversion of Lignocellulosic Biomass to High Octane Gasoline via Indirect Gasification and

Methanol Intermediate .................................................................................................................................146

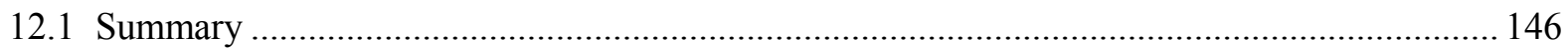

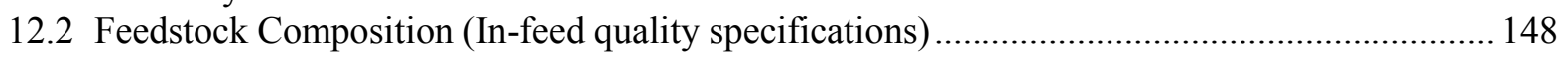

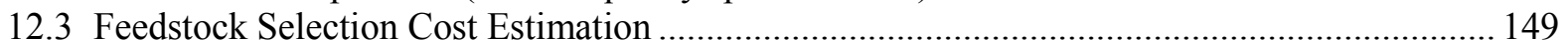

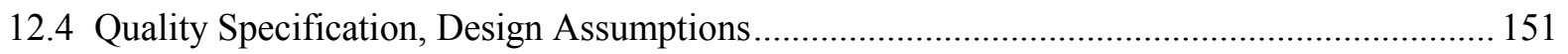

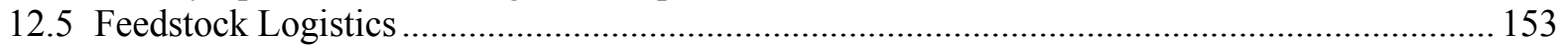

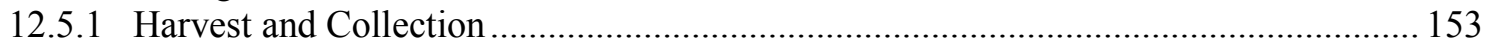

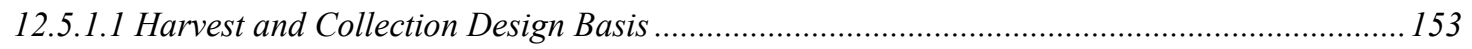

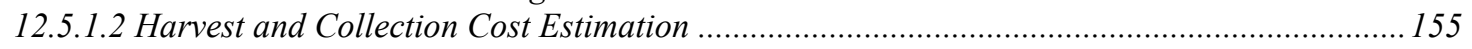

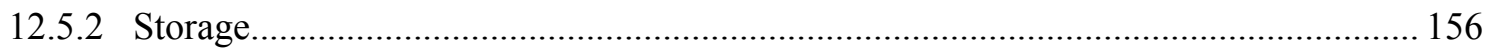

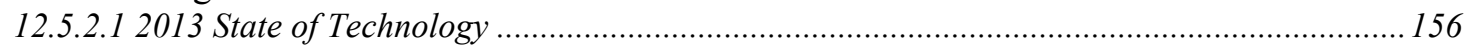

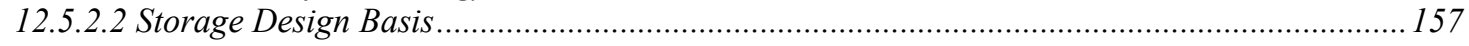

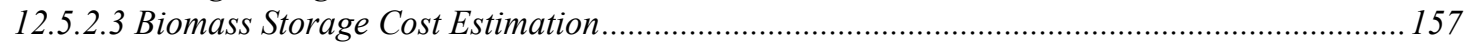

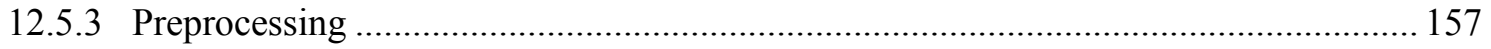

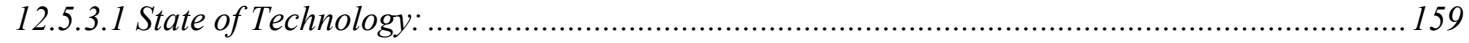

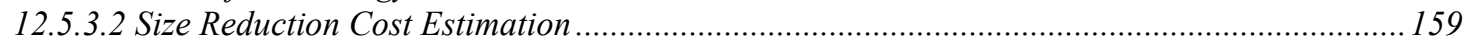

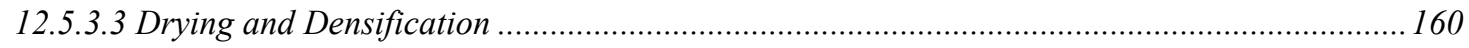

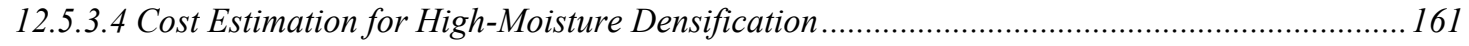

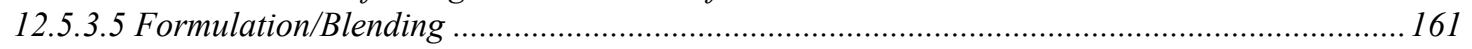

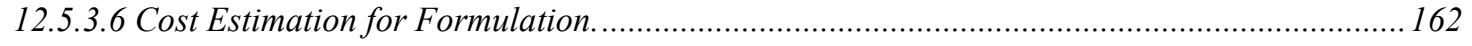

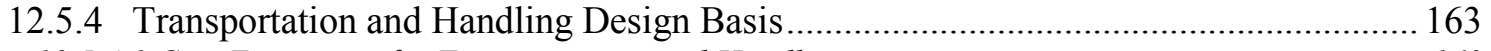

12.5.4.1 Cost Estimation for Transportation and Handling .............................................................. 163

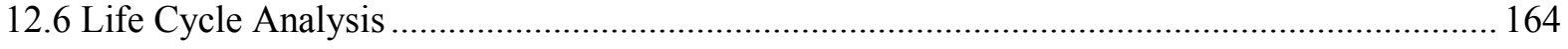

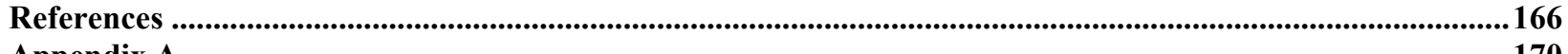

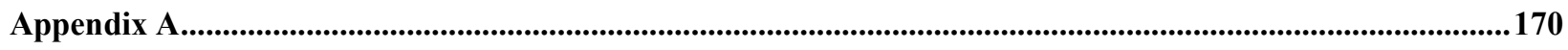




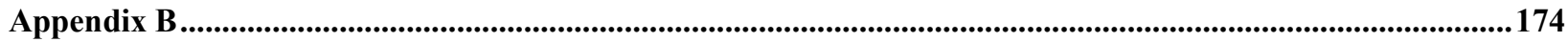

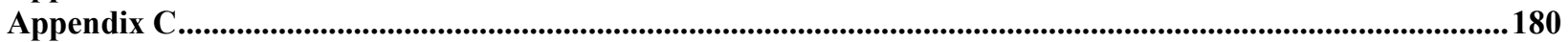

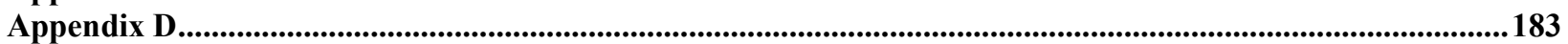

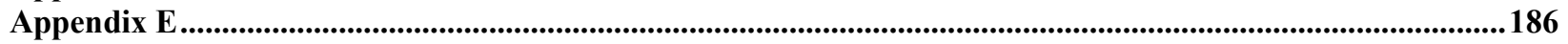




\section{Figures}

Figure 1. Total tons per county of available pulpwood at $\$ 60 /$ dry $\mathrm{T}$ farm gate price. Yellow circles show areas represented in the 2012 Conventional Design and the Relocated (2013) Design Case ${ }^{5}$.

Figure 2. Total tons per county of available corn stover at $\$ 40 /$ dry $\mathrm{T}$ farm gate price. Circles show areas represented in the 2012 Conventional Design and the Relocated (2013) Design Case.

Figure 3. Flow diagram of the 2012 Relocated (Baseline) Design Case to supply thermochemical conversion refineries ${ }^{12}$.....

Figure 4. Marginal and average farm gate costs versus supply quantities derived from BT2 data for the U.S. predicted out to $2022^{1}$. 32

Figure 5. U.S. Marginal farm gate prices projected for 2022 by POLYSYS ${ }^{1}$.

Figure 6. Historical pulpwood stumpage prices for the southern U.S. in $\$ /$ dry Ton (http://www.timbermart-south.com/prices.html).

Figure 7. National estimated pulpwood stumpage prices ${ }^{1}$

Figure 8. Ash content of corn stover bales from Stevens County, Kansas, that are collected using single pass baling and a variety of multi-pass methods, including two rakes, two balers, a mower, and a flail shredding windrower.

Figure 9. Ash content (bars) and yield (text) of corn stover bales from Stevens County, Kansas, show the impact of collection efficiency and windrowing equipment on yield and soil entrainment.

Figure 10. Dry matter loss of corn stover in laboratory storage conditions at fixed moisture contents 32 .

Figure 11. Change in moisture content of stacked corn stover bales in northern Iowa. Image depicts the variation in moisture content of a four-high column of bales stored outdoors for up to 9 months ${ }^{32}$.

Figure 12. Change in glucan and xylan over time as corn stover is stored in laboratory reactors ${ }^{32}$..........46

Figure 13. Dry matter loss and self-heating of 50\% initial moisture pine chips stored under aerobic conditions using laboratory scale reactors at INL.

Figure 14. Comparison of comminution capacities (tons of through put per operating hour) for woody biomass as a result of adding pneumatic transfer assist (PTS) ${ }^{38}$.

Figure 15. Change in moisture content during comminution using pneumatic transfer assist (PTS) 38

Figure 16. Ash content in various screen sizes for pinyon juniper biomass ${ }^{38} 41 \ldots \ldots \ldots \ldots \ldots \ldots \ldots \ldots \ldots \ldots \ldots \ldots . . . .50$

Figure 17. Particle-size distributions for five grinding scenarios ${ }^{41}$................................................... 51

Figure 18. Comparison of conventional, two-stage grinding and fractional milling ${ }^{41}$............................52

Figure 19. Grinding energy and throughput is highly dependent on screen size ${ }^{41}$...............................53

Figure 20. Hammer mill energy consumption is highly dependent on biomass moisture content

(INL PDU Data). 


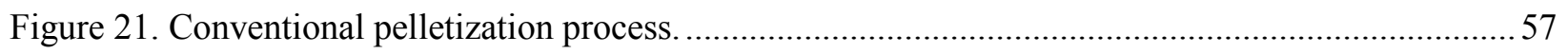

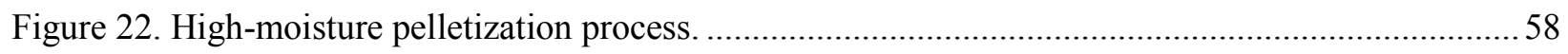

Figure 23. Conventional feedstock system for herbaceous lignocellulosic biomass. ...............................60

Figure 24. Advanced supply system designs (Advanced Uniform) follow the model of the current grain commodity supply system, which manages crop diversity at the point of harvest and/or depot, allowing all subsequent feedstock supply system infrastructure to be similar for all biomass resources ${ }^{4}$.

Figure 25. Comparison of individual and blended feedstock costs. A blend of $60 \%$ corn stover, $35 \%$ switchgrass, and 5\% municipal solid waste is needed to hit the $\$ 80$ feedstock cost target.

Figure 26. Resource selections for the 2017 Design Case to support biochemical conversion. Figure shows tonnages available at \$40/dry ton. Green represents higher amounts of tonnage available, red represents no available tonnage available at $\$ 40 /$ dry ton ${ }^{47}$.

Figure 27. The impact of dry matter loss on bale ash content and final conversion efficiency (based on a $30 \%$ initial moisture and $12 \%$ ash).

Figure 28. Dry matter loss of corn stover in the simulated storage conditions, with three air flows simulating three different oxygen availabilities. .73

Figure 29. Material flow in the 2017 Design Case that incorporates many improvements in preprocessing, including fractional milling, chemical preconversion, high-moisture densification, and formulation/blending.

Figure 30. Total tons per county of available pulpwood at $\$ 60 /$ dry $\mathrm{T}$ farm gate price. Yellow circles show areas represented in the 2012 Conventional Design and the Relocated (2013) Design Case ${ }^{5}$.

Figure 31. Conventional (left) and high-capacity grapple skidder (right) for transporting small diameter pulpwood from the forest to the landing. Photo credit: Auburn University High Tonnage Forest Biomass Project ${ }^{58}$.

Figure 32. Ash and moisture content of switchgrass harvested in Oklahoma, 2010 by Oklahoma State University. Error bars represent one standard deviation. Ash samples for October, December, and January are three samples comprised of six individual core samples composited.

Figure 33. Material flow in the 2017 Design Case that incorporates many improvements in preprocessing, including pneumatics, high-moisture densification, and formulation/blending.

Figure 34. Comparison of individual and blended feedstock costs. A blend of $60 \%$ corn stover, $35 \%$ switchgrass, and 5\% municipal solid waste is needed to hit the $\$ 80$ feedstock cost target.

Figure 35. Resource selections for the 2017 Design Case to support biochemical conversion. Figure shows tonnages available at $\$ 40 /$ dry ton. Green represents higher amounts of tonnage available, red represents no available tonnage available at $\$ 40 / \mathrm{dry}_{\text {ton }}{ }^{47}$.

Figure 36. The impact of dry matter loss on bale ash content and final conversion efficiency (based on a $30 \%$ initial moisture and $12 \%$ ash). 
Figure 37. Dry matter loss of corn stover in the simulated storage conditions, with three air flows simulating three different oxygen availabilities.

Figure 38. Material flow in the 2017 Design Case that incorporates many improvements in preprocessing, including fractional milling, chemical preconversion, high-moisture densification, and formulation/blending.

Figure 39. Total tons per county of available pulpwood at $\$ 60 /$ dry $\mathrm{T}$ farm gate price. Yellow circles show areas represented in the 2012 Conventional Design and the Relocated (2013) Design Case ${ }^{5}$.

Figure 40. Conventional (left) and high-capacity grapple skidder (right) for transporting small diameter pulpwood from the forest to the landing. Photo credit: Auburn University High Tonnage Forest Biomass Project ${ }^{58}$......

Figure 41. Ash and moisture content of switchgrass harvested in Oklahoma, 2010 by Oklahoma State University. Error bars represent one standard deviation. Ash samples for October, December, and January are three samples comprised of six individual core samples composited.

Figure 42. Material flow in the 2017 Design Case that incorporates many improvements in preprocessing, including pneumatics, high-moisture densification, and formulation/blending.

Figure 43 . Total tons per county of available pulpwood at $\$ 60 /$ dry $T$ farm gate price. Yellow circles show areas represented in the 2012 Conventional Design and the Relocated (2013) Design Case ${ }^{5}$.

Figure 44. Conventional (left) and high-capacity grapple skidder (right) for transporting small diameter pulpwood from the forest to the landing. Photo credit: Auburn University High Tonnage Forest Biomass Project ${ }^{58}$......

Figure 45. Ash and moisture content of switchgrass harvested in Oklahoma, 2010 by Oklahoma State University. Error bars represent one standard deviation. Ash samples for October, December, and January are three samples comprised of six individual core samples composited.

Figure 46. Material flow in the 2017 Design Case that incorporates many improvements in preprocessing, including pneumatics, high-moisture densification, and formulation/blending.

Figure B-1. The farm gate cost curves suggest that corn stover is the preferred feedstock because it is less expensive than switchgrass

Figure B-2. Accounting for quality (dockage) — shows that about 300,000 tons of switchgrass can be supplied at a lower cost than corn stover.

Figure B-3. A corn stover/switchgrass blend that will deliver at about $\$ 81 /$ dry T

Figure B-4. A minimum of 5\% MSW (at $\$ 1 /$ dry T) is needed to achieve the $\$ 80 /$ dry T cost target with a corn stover, switchgrass, and municipal solid waste blend...

Figure B-5. Comparison of individual and blended feedstock costs. A blend of $60 \%$ corn stover, $35 \%$ switchgrass, and 5\% municipal solid waste is needed to hit the $\$ 80$ feedstock cost target. 
Figure C-1. Sensitivity of dockage by altering feedstock ash content relative to the baseline ash content. of $4.9 \%{ }^{84}$.

Figure C-2. Sensitivity of the costs impacts to altering feedstock price relative to the base case of $58.50 \$$ dry $\mathrm{T}$ for a delivered feedstock with $10 \%$ ash ${ }^{84}$

Figure C-3. Total dockage based on shifting baseline ash content for a range of received materials at a feedstock price of $58.50 \$ /$ dry $\mathrm{T}^{84}$ 


\section{Tables}

Table E-1. Summary of assumptions underpinning progressive design implementations for biological conversion of sugars to hydrocarbons and catalytic conversion of sugars to hydrocarbons.

Table E-2. Summary of assumptions underpinning progressive design implementations for thermochemical conversion(Fast Pyrolysis and Hydro treating Bio-Oil Pathway, Thermochemical Pathways with In Situ and Ex Situ Upgrading of Fast Pyrolysis Vapors, Indirect Gasification) (INL 2017 Design Case). .........................................................

Table 1. Delivered woody feedstock composition and processing assumptions for the fast

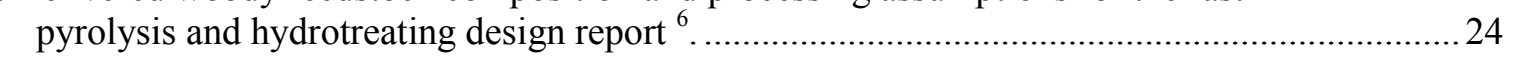

Table 2. Potential effect of higher biomass ash content..................................................................2

Table 3. Costs and specifications for woody feedstocks and blends (INL analysis). .............................28

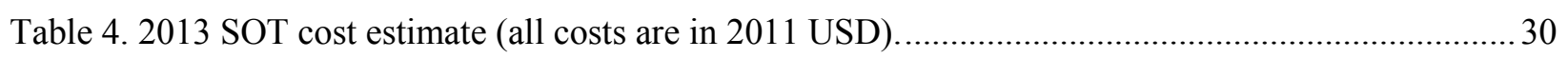

Table 5. Resource access cost estimate ${ }^{5}$ and INL Material Solid Waste (MSW Data) ............................3

Table 6. Mean total ash values and ranges for selected lignocellulosic biomass..................................... 40

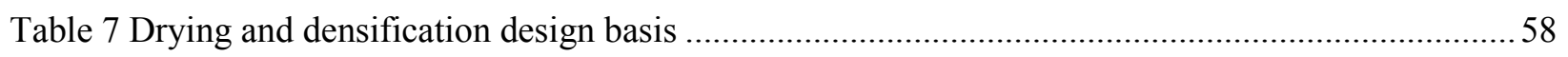

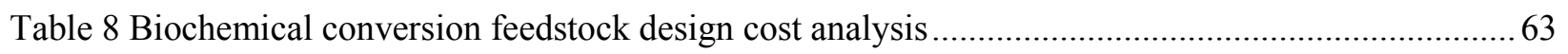

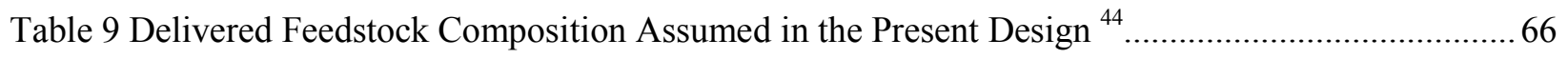

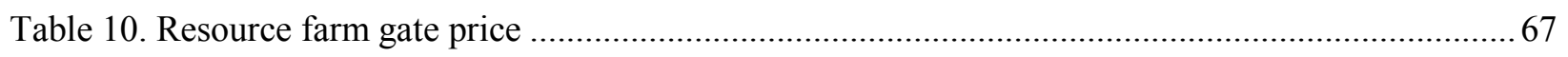

Table 11. Summary of assumptions underpinning progressive design implementations .........................68

Table 12.Technical targets for harvest and collection of herbaceous resources in the 2017 Design

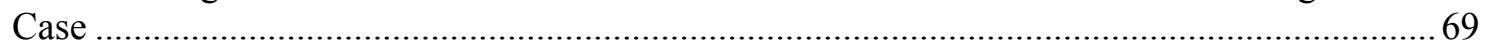

Table 13. Biomass harvest and collection cost estimates. .............................................................. 71

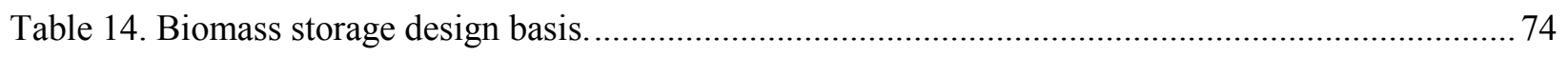

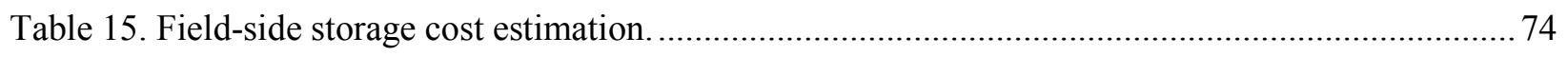

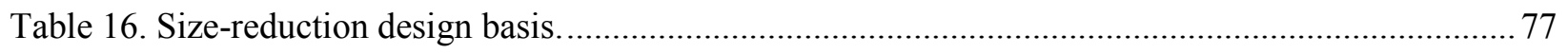

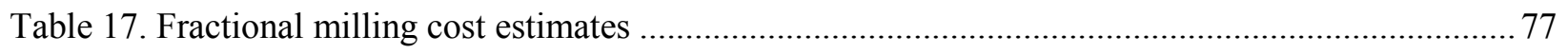

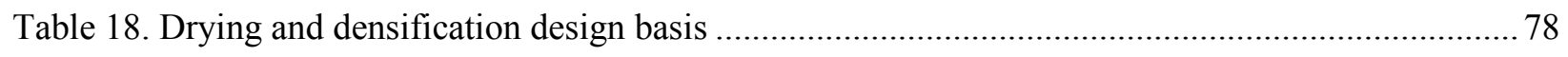

Table 19. Drying and densification cost estimates ............................................................................... 79

Table 20. Feedstock formulation/blending of ash and moisture contents * ............................................ 79

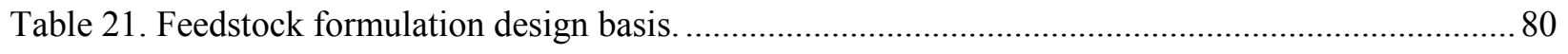

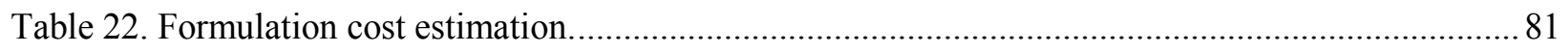

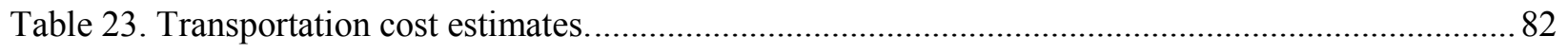

Table 24. Energy consumption for Biochemical conversion supply chain design. ................................. 83 


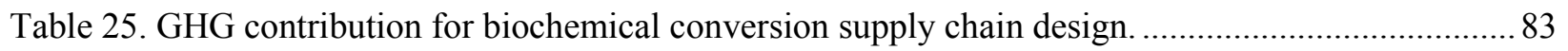

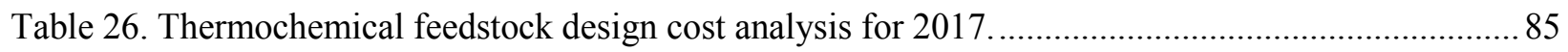

Table 27. Delivered woody feedstock composition and processing assumptions for the fast pyrolysis and hydrotreating design report ${ }^{6}$.

Table 28. Resource access cost estimate (U.S. DOE $2011^{5}$ and INL MSW Data).................................. 89

Table 29. Summary of assumptions underpinning progressive design implementations ${ }^{57}$....................... 90

Table 30. Biomass harvest and collection cost estimates derived from INL analysis ............................94

Table 31. Technical targets for biomass field storage of resources in the 2017 Design Case. ..................95

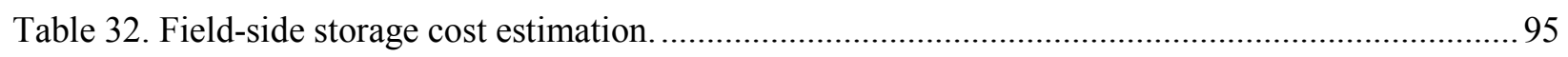

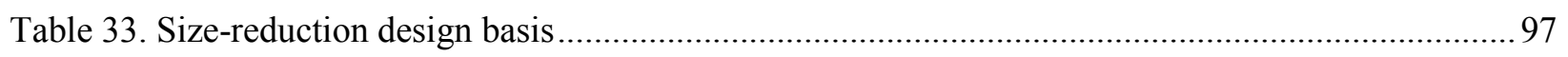

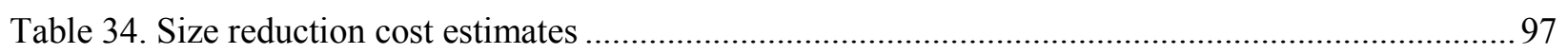

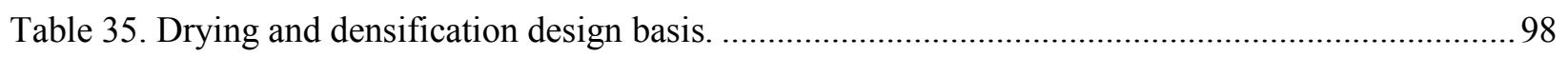

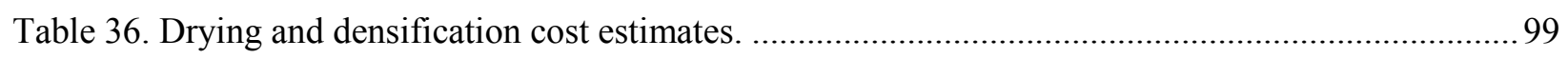

Table 37. Feedstock formulation/blending of ash and moisture contents* ............................................ 99

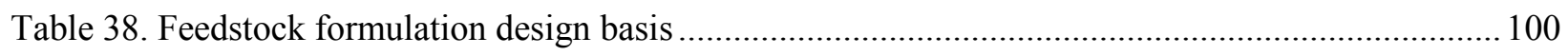

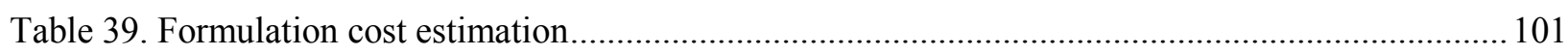

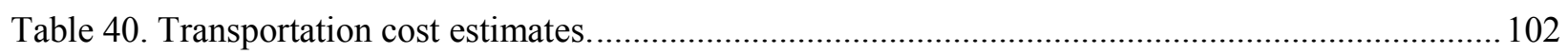

Table 41. Energy Consumption for Thermochemical conversion supply chain design.......................... 102

Table 42. GHG contribution for thermochemical conversion supply chain design............................... 103

Table 43. Biochemical conversion feedstock design cost analysis...................................................... 105

Table 44. Delivered Feedstock Composition Assumed in the Present Design ${ }^{44}$.................................. 108

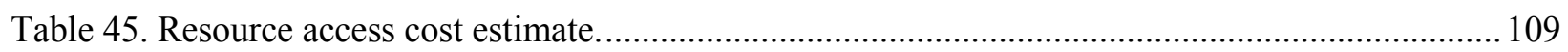

Table 46. Summary of assumptions underpinning progressive design implementations. ...................... 110

Table 47. Technical targets for harvest and collection of herbaceous resources in the 2017 Design

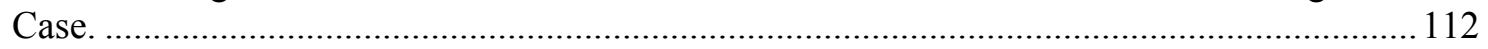

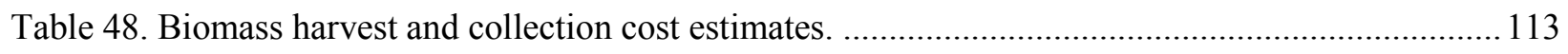

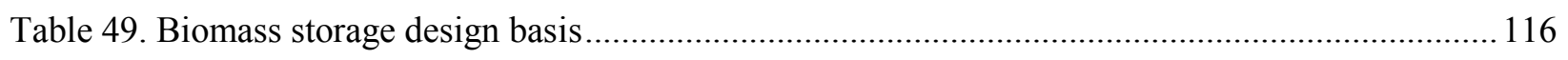

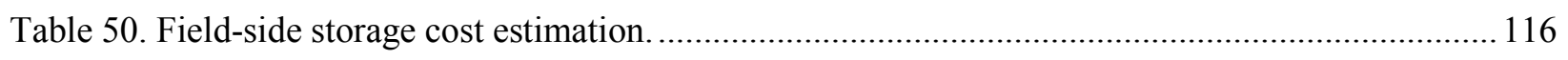

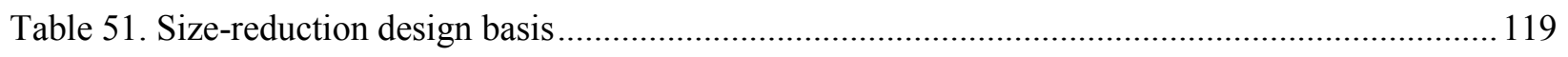

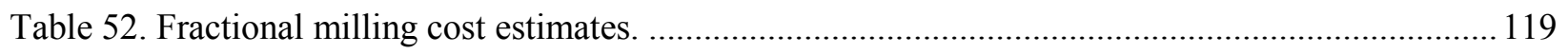

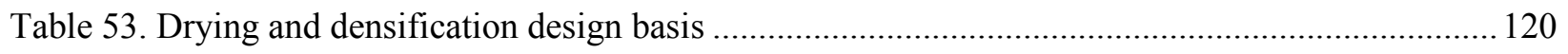

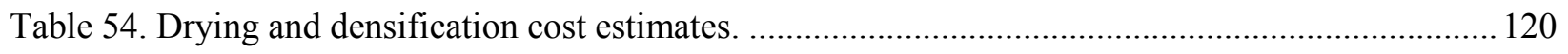

Table 55. Feedstock formulation/blending of ash and moisture contents*......................................... 121

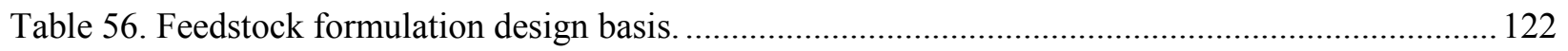




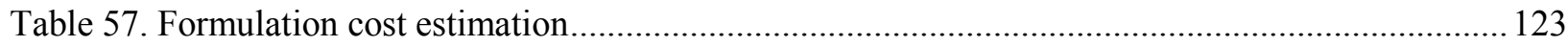

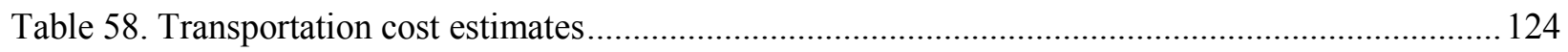

Table 59. Energy consumption for Biochemical conversion supply chain design. ............................... 124

Table 60. GHG contribution for biochemical conversion supply chain design. .................................... 125

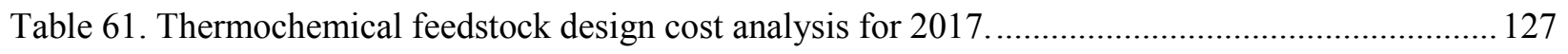

Table 62. Delivered woody feedstock composition and processing assumptions for the In Situ and

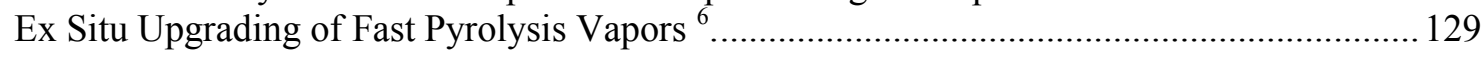

Table 63. Resource access cost estimate (U.S. DOE 2011 ${ }^{5}$, and INL MSW Data)............................. 131

Table 64. Summary of assumptions underpinning progressive design implementations ${ }^{57}$..................... 132

Table 65. Biomass harvest and collection cost estimates derived from INL analysis. ........................... 136

Table 66. Technical targets for biomass field storage of resources in the 2017 Design Case. ................ 137

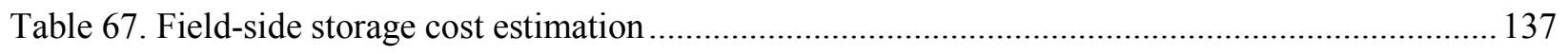

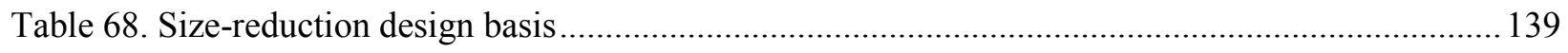

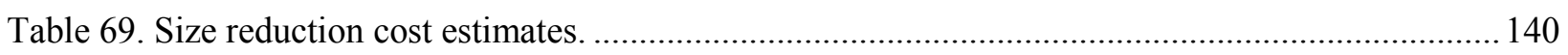

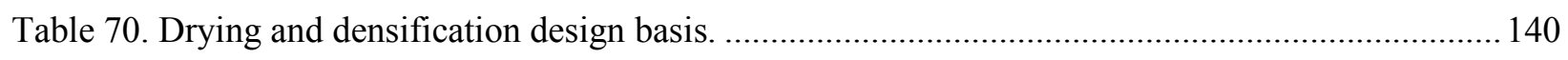

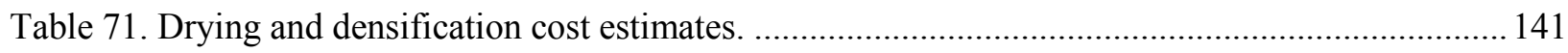

Table 72. Feedstock formulation/blending of ash and moisture contents* .......................................... 142

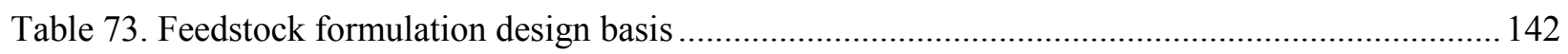

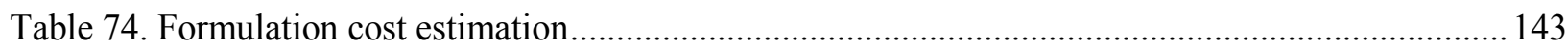

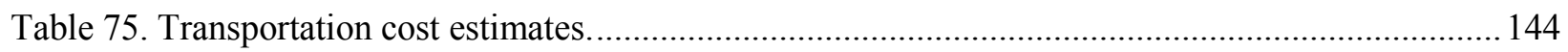

Table 76. Energy Consumption for Thermochemical conversion supply chain design.......................... 145

Table 77. GHG contribution for thermochemical conversion supply chain design............................... 145

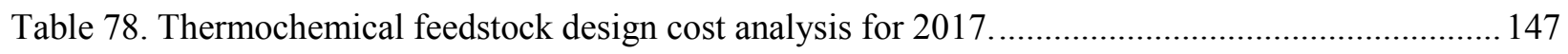

Table 79. Delivered woody feedstock composition and processing assumptions for the fast

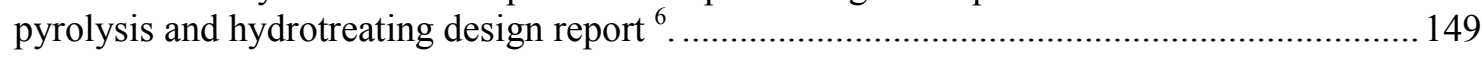

Table 80. Resource access cost estimate (U.S. DOE $2011^{5}$, and INL MSW Data).............................. 151

Table 81. Summary of assumptions underpinning progressive design implementations ${ }^{57}$................... 152

Table 82. Biomass harvest and collection cost estimates derived from INL analysis. .......................... 156

Table 83. Technical targets for biomass field storage of resources in the 2017 Design Case. ................ 157

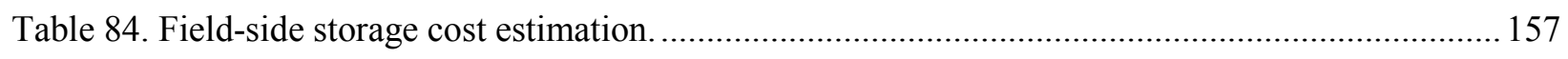

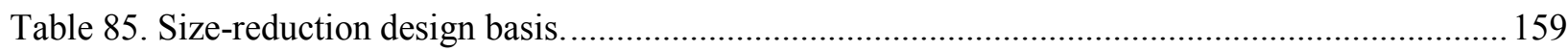

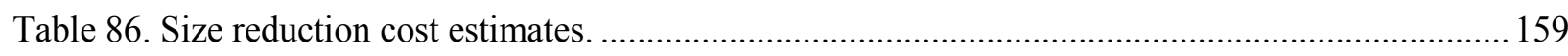

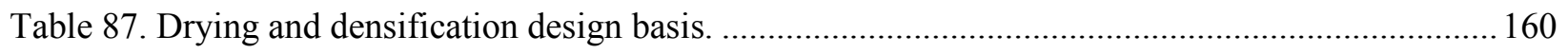

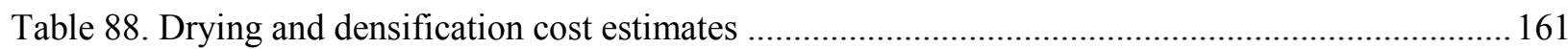


Table 89. Feedstock formulation/blending of ash and moisture contents*........................................ 161

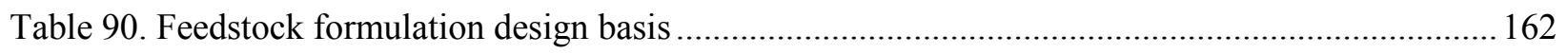

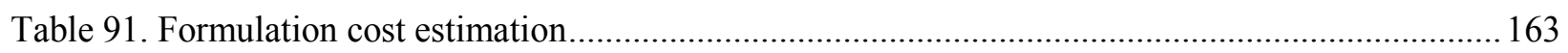

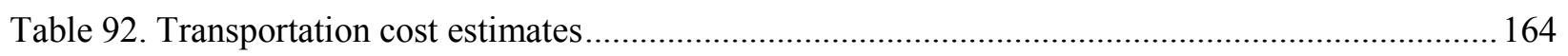

Table 93. Energy Consumption for Thermochemical conversion supply chain design.......................... 164

Table 94. GHG contribution for thermochemical conversion supply chain design................................ 165

Table A-1 Potential C\&D available in select counties in western South Carolina .................................. 170

Table A- 2 National average municipal solid waste composition......................................................... 171

Table A- 3. Per capita generation rates for various fractions of municipal solid waste and construction and demolition waste (lb/person/day)........................................................... 172

Table A- 4. Physical parameters of solid waste .............................................................................. 173

Table D-1. Expected unit operations and assumptions for the application of a drain and fill leaching system for the removal of soluble ash from biomass in a feedstock depot................ 184 


\section{List of Acronyms}

$\begin{array}{ll}\text { C\&D waste } & \text { Construction and demolition waste } \\ \text { Dry T } & \text { Dry Ton } \\ \text { MSW } & \text { Municipal Solid Waste } \\ \text { BETO } & \text { Bioenergy Technology Office } \\ \text { BT2 } & \text { Billion Ton Study Update } \\ \text { DMT } & \text { Dry Matter Ton (U.S. short ton) } \\ \text { DOE } & \text { U.S. Department of Energy } \\ \text { GGE } & \text { Gallon Gasoline Equivalent } \\ \text { I\&D } & \text { Interest and Depreciation (costs) } \\ \text { IH\&T } & \text { Insurance Housing and Taxes (costs) } \\ \text { INL } & \text { Idaho National Laboratory } \\ \text { MC } & \text { Moisture Content } \\ \text { MESP } & \text { Minimum Ethanol Selling Price } \\ \text { ORNL } & \text { Oakridge National Laboratory } \\ \text { PDU } & \text { Process Demonstration Unit (at INL) } \\ \text { R\&M } & \text { Repairs and Maintenance (costs) } \\ \text { TEA } & \text { Techno-Economic Analysis } \\ \text { LCF } & \text { Least Cost formulation } \\ \text { PNNL } & \text { Pacific Northwest National Laboratory } \\ \text { NREL } & \text { National Renewable Energy Laboratory } \\ & \end{array}$




\section{2017 Design Case}

The success of the earlier logistic pathway designs (Biochemical and Thermochemical) from a feedstock perspective was that it demonstrated that through proper equipment selection and best management practices, conventional supply systems (referred to in this report as "conventional designs," or specifically the 2012 Conventional Design) can be successfully implemented to address dry matter loss, quality issues, and enable feedstock cost reductions that help to reduce feedstock risk of variable supply and quality and enable industry to commercialize biomass feedstock supply chains. The caveat of this success is that conventional designs depend on high density, low-cost biomass with no disruption from incremental weather. In this respect, the success of conventional designs is tied to specific, highly productive regions such as the southeastern U.S. which has traditionally supported numerous pulp and paper industries or the Midwest U.S for corn stover.

The goal of the 2017 Logistics Design Case is to increase availability of affordable biomass beyond only highly productive resource areas. The 2017 programmatic target is to supply the conversion facility with a feedstock that meets the conversion feedstock specifications at a total delivered cost of $\$ 80 /$ dry $T$. This design document describes a feedstock logistics design capable of achieving this goal, discusses the limitations of the conventional supply systems when applied outside of highly productive resource areas and shows how these limitations can be resolved through integration of multiple types of feedstocks, clear definition of biomass quality specifications, and technology advancement in logistics and preprocessing.

The $\$ 80 /$ dry T target encompasses a total delivered feedstock cost, including both grower payment and logistics, and meeting all conversion in-feed quantity and quality targets. The 2012 $\$ 55 /$ dry $T$ target for the thermochemical conversion pathway and the $\$ 35 /$ dry $\mathrm{T}$ biochemical conversion pathway target included only logistics costs and only included passive quality control methods. An estimated grower payment associated with the 2012 Conventional Design was $\$ 15.20$ /dry T based on the break-even cost of production ${ }^{1}$. Adding grower payment and logistics, the total delivered feedstock cost was $\$ 70.20 /$ dry $\mathrm{T}$ for thermochemical conversion and $\$ 50.20$ /dry T for biochemical conversion in 2007 dollars. Translating the $\$ 70.20 /$ dry T to 2011 dollars, the total delivered feedstock cost of the 2012 Conventional Design scales to about $\$ 80 /$ dry T. This demonstrates that for a conventional supply system it is possible to deliver feedstock to various conversion pathways for $\$ 80 /$ dry $T$ or less, but only for of tightly coupled set of designs that require high quality, low moisture harvested material. First, the 2012 Conventional Design assumed field drying the material. Next, it was assumed that waste heat from the conversion facility would be available for further drying requirements. And finally, the design assumed that biomass was available in high yield regions, minimizing transportation distances. Each of these assumptions limits the size and location of conversion facilities and assumes that weather will not impact biomass quality. Achieving the goals of the 2017 Design Case will require innovative solutions and significant technological advancements as pivotal assumptions within the 2012 Conventional Design are removed.

This report is intended to describe the feedstock logistic pathway for supplying biomass to a conversion facility. The logistic designs are not conversion pathway specific, but discuss in general the methods and processes necessary to stabilize and densify large volumes of biomass 
that can then be delivered to the specific conversion pathways at a specified quality level. Sections 8-11 will then outline the costs and quality impacts for the current conversion pathways. Feedstock design reports associated with alternate hydrocarbon pathways of BETO will be published subsequent to this report.

\section{Limitations of Conventional Supply System Designs}

Conventional design is based on the supply system where corn stover or other herbaceous residues are procured through contracts with local growers, harvested, stored at field side, and delivered in bale format to the conversion facility. A detailed description of conventional supply system is provided in section 7.1. Conventional designs are the backbone of an emerging biofuels industry. In fact, conventional designs have been successfully implemented by pioneer (1st of a kind, small capacity) biorefineries currently in operation. However, conventional supply systems have limitations ${ }^{2}$, that prohibit them from being broadly implemented to access the diverse set of resources needed to support a national biorefinery capability. These limitations, including biomass availability, feedstock quality and high costs, are discussed in this section.

The viability of the 2012 Conventional Design is rooted in areas that have a concentrated supply of easily accessible, and low-cost biomass resources (i.e., termed highly productive resource areas in this 2017 Design Case). Moving outside of these select regions, the feedstock supply system must be adapted to accommodate a different supply-demand dynamic brought about by changing cost, quality, and conversion facility size constraints. When located outside highly productive areas, biorefineries that rely on conventional designs are likely to be small due to the high cost of transportation of low density biomass, limiting their ability to achieve economies of scale, because feedstock costs and risks are likely to be prohibitive ${ }^{4}$.

Biomass is highly variable in quality (e.g., ash, moisture, and particle size). Conventional systems can only address feedstock quality indirectly through passive controls such as resource selection or best management practices, and harvest technique. When positioned in a highly productive area, biorefineries can be selective in contracting only those feedstocks that meet their specifications. Best management practices also can be used to reduce issues of moisture and ash, but they will not eliminate them. Additional work needs to be done both on the conversion side as well as the feedstock side to identify additional quality specifications and to align them with the various feedstocks which match up with the conversion process including woody, herbaceous residues, energy crops and various municipal solid wastes (MSW). The new quality specifications could include breaking down total ash by species (e.g. potassium and other alkali), cellulose, hemicellulose, lignin, and other extractives. Each conversion pathway will have a select set of quality specifications that may very well dictate regions of the country that are better aligned to supply feedstocks.

Two requirements that distinguish the 2017 Design Case from the 2012 Conventional Design are first expansion beyond highly productive resource areas and second adherence to feedstock specifications. These requirements are discussed in detail in the following subsections. 


\subsection{Expansion Beyond Highly Productive Regions}

Expansion beyond highly productive resource areas has significant implications to the feedstock supply chain. Sparse areas, whether due to reduced yields and/or higher dispersion, typically increase feedstock logistics costs. Higher harvest and collection costs are incurred due to the need to spread machinery ownership costs over fewer tons of biomass, or the need to cover more acres for the same quantity of biomass. Additionally, lower resource yields increase the supply radius and biomass transportation distances. Under the 2012 Conventional Design, higher yield areas allow refinery to be selective on the resource that they access.

Consider, for example, pulpwood farm gate (farm gate prices include harvest, collection and revenue to the owner) prices depicted in Figure 1. This resource map illustrates a county-level resource assessment of pulpwood farm gate at $\$ 60$ /dry T prices (this includes grower payment, harvest, collection, and chipping costs). Farm gate price data were extracted from The Billion Ton Update (BT2) ${ }^{5}$ data supplied from Oak Ridge National Laboratory.

The cost competitiveness of the 2012 Conventional Design was demonstrated ${ }^{3}$ in the scenario located in southern Alabama, a high biomass yielding area (2012 Design in Figure 1). We further suggest, based on the consistency of farm gate (i.e., landing) prices shown in this map, that the 2012 Conventional Design can be deployed cost effectively in South Carolina (2013 Design in Figure 1).

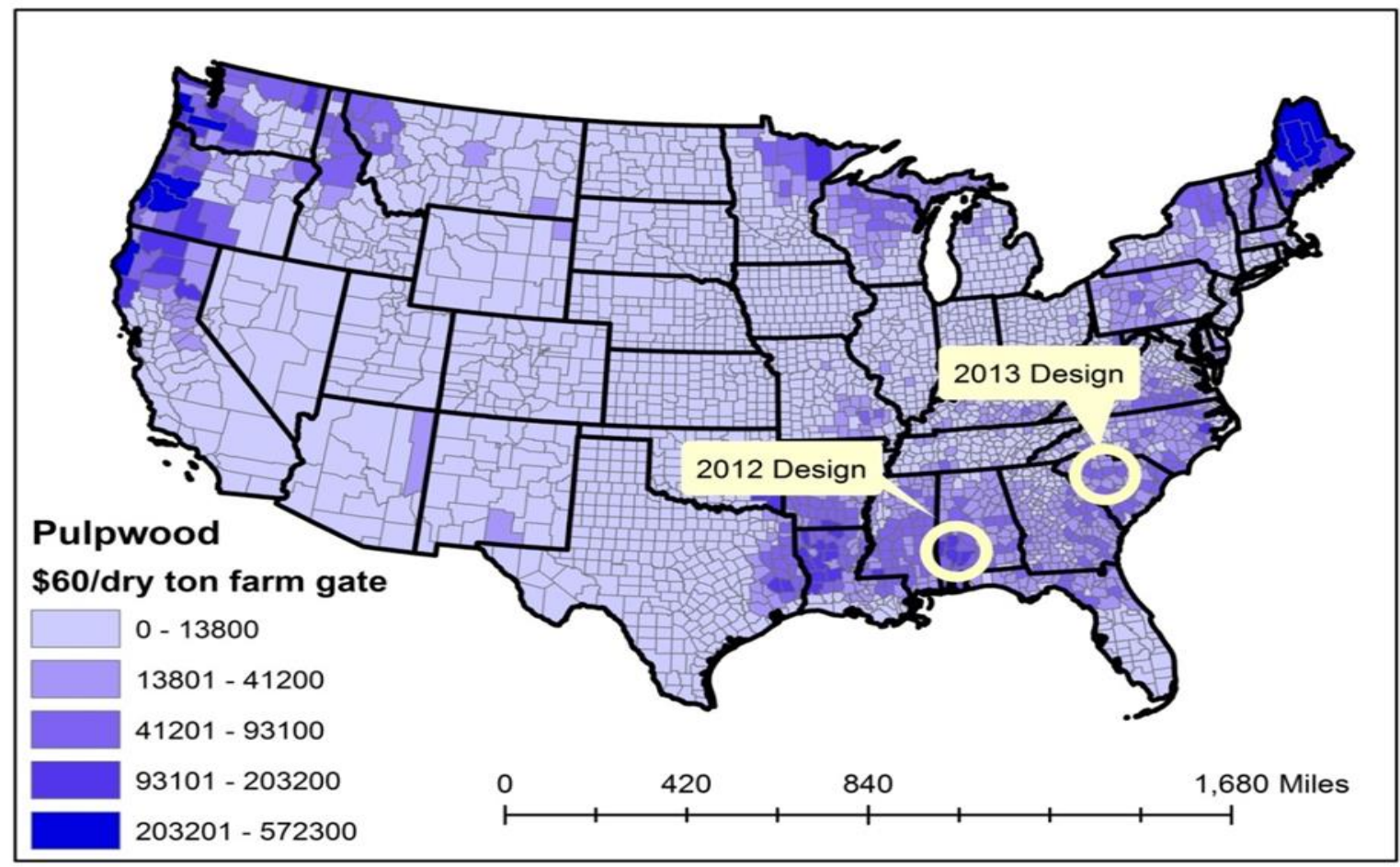

Figure 1. Total tons per county of available pulpwood at $\$ 60 /$ dry $\mathrm{T}$ farm gate price. Yellow circles show areas represented in the 2012 Conventional Design and the Relocated (2013) Design Case 5 . 
The map depicts a fairly steep gradient where resource available at $\$ 60 /$ dry $\mathrm{T}$ farm gate price rapidly decreases (lighter colors) for areas outside the southern area that traditionally supported a thriving pulp and paper industry. Significant county-to-county fluctuations in available resources are seen as well. In these areas, as in the scenario depicted in western South Carolina, sufficient pulpwood exists to support an 800,000 ton per year biorefinery; however resource selectivity to passively mitigate quality will be constrained due to the limited amount of biomass (i.e., selecting only the higher quality material and leaving the low quality material behind). In this scenario, a more dispersed pulpwood resource, due to lower yields in these regions, also results in increased harvest, collection, and transportation costs compared to the lower-cost scenario.

To reinforce this concept and to expand the analysis to other feedstocks, Figure 2 depicts farm gate price for corn stover in the Midwest for two areas. The first is in Iowa where corn yields are high and the second area moves to western Kansas with lower yields. The map also depicts (as in the pulpwood case) a fairly steep gradient where at $\$ 40 /$ dry $T$ farm gate price resources rapidly decrease toward the fringes of the Corn Belt. Significant county-to-county fluctuations in resources are seen within this fringe zone as well. In these areas, as in the scenario depicted in western Kansas, ample corn stover exists to support large-scale biorefineries however; feedstock access costs alone may be more than double the Corn Belt prices. A more dispersed corn stover resource, due to lower yields in these regions, also results in increased harvest, collection, and transportation costs compared to the lower-cost scenario.

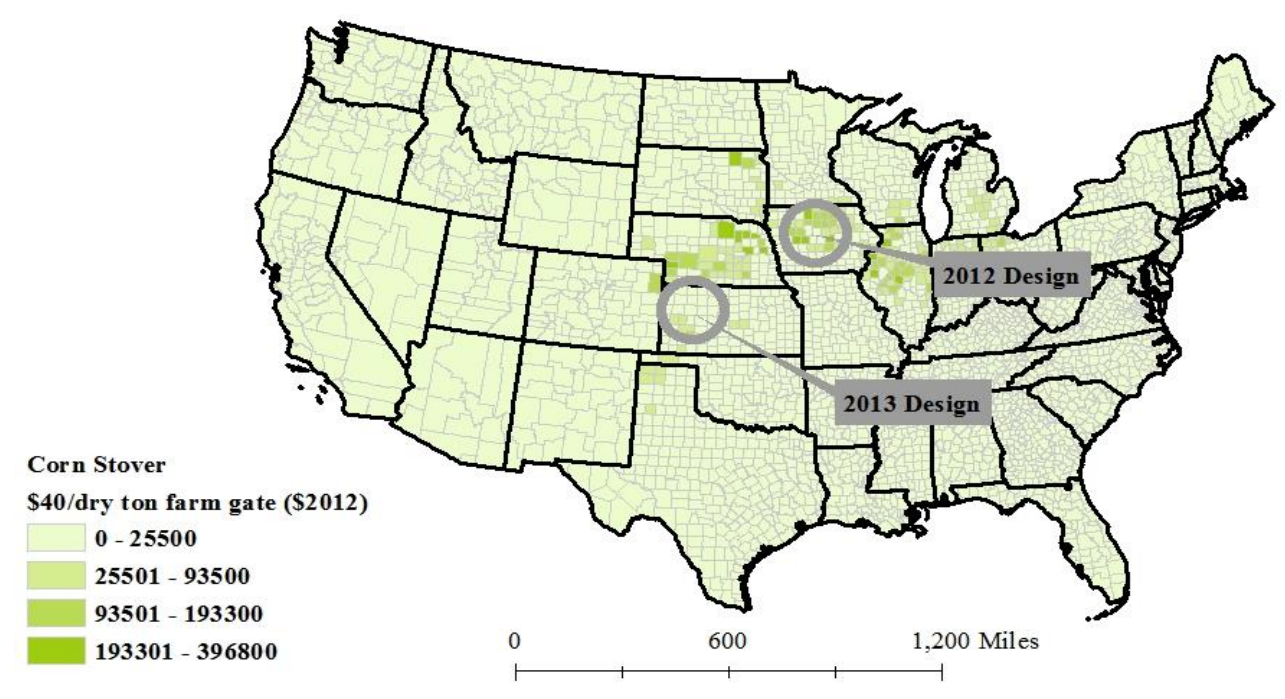

Figure 2. Total tons per county of available corn stover at $\$ 40 /$ dry $T$ farm gate price. Circles show areas represented in the 2012 Conventional Design and the Relocated (2013) Design Case.

\subsection{Feedstock Quality Specifications}

Techno-economic models often involve process simulations wherein mass and energy balances converged across a number of unit operations to assess the entire system as a whole. Feedstock compositions and heating values are required inputs to these balances. The fast pyrolysis and hydrotreating conversion pathway (Section 9) feedstock specifications are shown in Table 1. 
These assumptions were based on the composition of low ash, woody biomass delivered at $30 \% \mathrm{wt}$ moisture ${ }^{6}$. Currently within the feedstock logistic system the only quality specifications that are actively being managed are ash, particle size and moisture. Other components, such as lignin, low and high heating values, may be included in future revisions as their quality impacts are identified. A combination of passive methods such as, selective biomass selection, applying best management practices that preserve material, and harvest practices that minimize introduced ash (soil) were implemented in the 2012 Conventional Design. However, quality "specifications" were not actively enforced in the 2012 Conventional Design. In other words, the specifications were acknowledged and attempts were made to match up the biomass with the specifications, but no active management was installed to enforce expectations and it was assumed that the passive controls were enough to insure the quality specifications were met.

Table 1. Delivered woody feedstock composition and processing assumptions for the fast pyrolysis and hydrotreating design report ${ }^{6}$.

\begin{tabular}{|lc|}
\hline Component & $\begin{array}{c}\text { Composition } \\
\text { (dry wt. \%) }\end{array}$ \\
\hline Carbon & 50.94 \\
\hline Hydrogen & 6.04 \\
\hline Nitrogen & 0.17 \\
Sulfur & 0.03 \\
Oxygen & 41.90 \\
\hline Ash & $0.90-1.0$ \\
\hline Heating Value (Btu/lb) & $8,601 \mathrm{HHV}$ \\
\hline Moisture (Bulk Wt. \%) & $7,996 \mathrm{LHV}$ \\
\hline Particle Size (inch) & 10.0 \\
\hline
\end{tabular}

Table 1 illustrates the feedstock conversion specifications for one particular conversion pathway, fast pyrolysis and hydrotreating. Each conversion pathway under consideration has its own set of feedstock conversion specifications. The impacts of the in-feed specifications for each conversion pathway are addressed later in this report in chapters specific to each pathway. The 2017 Design Case introduces the expectation that the feedstock supply system will be held accountable to deliver feedstocks that meet these quality assumptions.

The passive approaches (i.e., biomass selection and best management practices) implemented in the 2012 Conventional Design Case are not sufficient to guarantee feedstock specifications. Further, passive approaches to feedstock quality assurance restrict feedstock availability and producer participation, and ultimately increase feedstock costs and supply risk to biorefineries by making them dependent on limited specific feedstocks. Case in point, there may be years where due to rainy weather biomass does not undergo any field drying, thereby increasing biomass 
moisture which results in decreased harvest yields, increased dry matter loss, transportation costs, grinding costs and drying costs each of which increase the cost to the biorefinery.

The solution to be implemented in the 2017 Design Case still includes biomass selection and best management practices; however, this design also introduces active quality controls into the feedstock supply system. The 2017 Design Case approach enables access to the vast and diverse biomass resources available to support a national biofuels production capacity, while assuring strict adherence to biorefinery quality specifications.

A significant challenge for implementing active quality controls is that adding in additional processes adds cost to an already cost-constrained system. Therefore, the insertion of active controls into the 2017 Design Case must balance the cost/benefit of mitigation in the feedstock supply system and the cost of further biorefinery processing of off-spec feedstock.

In commercial practice, this normalization function is implemented through a dockage fee. A dockage fee is the penalty a feedstock supplier incurs from payment by the biorefinery for delivery of off-specification feedstock. The dockage fee is established based on the additional cost the biorefinery incurs to process off-specification feedstock; the dockage fee is subtracted from the contracted feedstock payment the biorefinery agreed to pay a supplier. If the predelivery cost of mitigation by the feedstock supplier exceeds the dockage fee, the dockage fee is the lowest cost option; otherwise, the feedstock supplier could implement corrective strategies to avoid the dockage penalty and remain economically competitive. For example, if ash removal is required to meet the biorefinery feedstock quality specification and mitigation within the feedstock supply system costs the supplier $\$ 15 /$ dry T, but the biorefinery is able to mitigate the ash for $\$ 10 /$ dry $T$, the feedstock supplier may choose to accept the $\$ 10 /$ dry $T$ dockage fee rather than implement ash reduction, for a net $\$ 5 /$ dry $T$ savings. For further discussion on dockage see Appendix C

Implementation of a dockage-based quality assurance approach, much like in the grain industry, requires accurate assessment of the cost/specification relationship(s), the practicality and cost effectiveness of the mitigation approach, and the availability of rapid and accurate analytical methods for measurement of the specifications at the point of sale. The following list describes an initial approach to establishing dockage for moisture and ash content.

\subsubsection{Moisture Specification}

For some conversion processes, i.e. thermochemical processing, feedstock moisture content has a strong influence on the process economics. High quality heat in the reactor will be required to evaporate the extra moisture which can be a significant penalty on the process depending on how it is configured ${ }^{7}$.The reality is that the moisture has a high impact on both the feedstock supply system as well as the conversion process. In addition to its implications on storage stability, biomass moisture content can significantly affect transportation, preprocessing, and feedstock handling ${ }^{8}$. These logistics-related costs are discussed in Section 3. Since the logistics supply system is designed to meet the moisture target there will not be a dockage assessed by the biorefinery for excessive feedstock moisture content. 


\subsubsection{Ash Specification(s)}

Feedstock ash content has a big impact on liquid yields for most conversion processes. Ash in this context is a combination of inorganic material that has two parts: (1) the mineral matter taken up from the soil and retained as part of the plant or tree; (aka physiological ash), and (2) inorganic material that was collected with the biomass during harvesting/collection (e.g. soil matter, dirt, etc.). Both parts are not converted during conversion and represent solid input that goes through the process and requires disposal. This extra inert mass causes a decrease in conversion efficiency and creates a waste stream that needs additional removal costs. Current predicted disposal costs are $\$ 18 /$ T for pyrolysis $^{9}$. The cost for other conversion processes may be different.

The goal of implementing improved feedstock supply system design is to minimize the inorganic material collected during harvest/collection and not address the physiological ash in the plants. In future supply systems, advanced preprocessing may be required to address physiological ash. Another option worth pursuing is that feedstock with higher ash is purchased at a low enough price to compensate for the losses in fast pyrolysis liquid yield or an added cleanup process to bring it in on specification. INL, in collaboration with NREL and PNNL, are researching the benefits/costs of both methods. Table 2 illustrates the effect of ash content on fast pyrolysis liquid yield. Additionally, feedstock ash content represents an additional variable operational cost to the biorefinery, because it reduces pretreatment efficacy, increases wear in handling and feeding systems, accumulates as a waste stream that requires disposal, and increases water treatment costs ${ }^{10}$. While ash entrained in the liquid could impact downstream catalysis, it is unknown at this time if the ash is soluble or attached to the char. There is a filtration step prior to condensed-phase upgrading that can help reduce the impacts. If the ash is soluble, it may impact the catalyst life.

Limited understanding is known about the efficiency of upgrading high-ash feedstock pyrolysis oils. Preliminary efforts indicate that oil produced from high-ash feedstocks actually performs better during hydrotreating. This would indicate that the loss of efficiency during pyrolysis is balanced by improved efficiency during hydrotreating. This might actually be an improved scenario because lost efficiency during hydrotreating is typically related to small molecular weight acids that consume hydrogen before being lost to the fuel gas ${ }^{11}$. 
Table 2. Potential effect of higher biomass ash content ${ }^{6}$.

\begin{tabular}{|ccc|}
\hline Cost and Consumption & $\begin{array}{c}\text { Base Case } \\
\text { with } 0.9 \% \text { wt Ash }\end{array}$ & $\begin{array}{c}\text { Base Case } \\
\text { with } 1.9 \% \text { wt Ash }\end{array}$ \\
\hline $\begin{array}{c}\text { Fuel yield, } \\
\text { gal/dry T. biomass }\end{array}$ & 84 & 75 \\
$\begin{array}{c}\text { Natural gas } \\
\text { usage, scf/gal }\end{array}$ & 19.3 & 5.9 \\
\hline $\begin{array}{c}\text { H2 demand, } \\
\text { MMscfd }\end{array}$ & 44.5 & 40 \\
TCI, million \$ & 700 & 672 \\
\hline MESP, \$/gge & 3.39 & 3.55 \\
\hline
\end{tabular}

Note: TCI (Total Cost Indicator), scf $=$ standard cubic feet

Crop residues forest thinnings, logging residues, and construction and demolition (C\&D) wastes are low cost resources to procure, but also have unfavorable quality specifications, specifically ash content. It should be noted that the ash type and quantity may have an effect on the yield of fast pyrolysis oil as certain ash constituents can cause an increase in the gas production at the expense of condensable liquids; however more research is needed to understand the impacts of ash on conversion.

Because these biomass resources are low cost, supply chains that include active ash management preprocessing unit operations can be purchased. Prior to preprocessing, certain resources can be blended to reduce the overall percentage of ash and moisture, thereby reducing the costs and severity needed to reduce the ash to in-feed specifications. Table 3 shows an example formulation. 
Table 3. Costs and specifications for woody feedstocks and blends (INL analysis).

\begin{tabular}{|c|c|c|c|}
\hline Feedstock & $\begin{array}{c}\text { Reactor Throat } \\
\text { Feedstock Cost } \\
(\$ / \text { dry } T)^{2}\end{array}$ & $\begin{array}{l}\text { Formulation } \\
\text { Fraction }(\%)\end{array}$ & $\begin{array}{c}\% \text { Ash Delivered } \\
\text { to Throat of } \\
\text { Conversion Reactor }\end{array}$ \\
\hline Pulp & 99.49 & 45 & 0.5 \\
\hline Wood Residues ${ }^{1}$ & 67.51 & 32 & 1 \\
\hline Switchgrass & 66.68 & 3 & 4.0 \\
\hline C\&D Wastes & 58.12 & 20 & 1.0 \\
\hline $\begin{array}{l}\text { Formulation } \\
\text { Totals }\end{array}$ & 80.00 & 100 & $<1$ \\
\hline
\end{tabular}

${ }^{1}$ residues do not include costs for harvest and collection; they are moved to landing while attached to the merchantable portion of the tree (for example, timber or pulpwood)

2 includes ash mitigation

The C\&D wastes are incorporated because of its low access fee cost and assumed low ash content. C\&D wastes were limited in quantity due to the uncertainty of available supplies. Current research shows significant quantities of C\&D, but there are uncertainties with EPA qualifying C\&D waste for credit for RIN's and competition from other markets. This is only an example; the actual blends will be regionally based designs that take advantage of local feedstocks and their biomass characteristics. Additionally the ability to blend feedstocks to a specification has the potential to reduce some of the risks associated with the seasonality of feedstocks. 


\section{Moving beyond the 2012 Conventional Design}

With the 2012 Conventional Design Case (e.g. thermochemical conversion pathway) located in a highly productive pulpwood production area, the main constraint of the design was that the biorefinery could be selective in contracting pulpwood that was $<1 \%$ ash content (Table 1 ). In the 2013 State of Technology (SOT), the assumption that the biorefinery can be selective is unlikely because there will most likely not be enough resource available to maintain the volumes required.

The feedstock supply system unit operations modeled in the 2013 SOT case for the thermochemical conversion path are shown in Figure 3. These unit operations are identical to those in the 2012 Conventional Design ${ }^{12}$, with the exception of the removal of the waste heat drying. The new assumption is that waste heat drying will not be available to support feedstock drying but will be used elsewhere inside the conversion facility. The details of these unit operations are discussed in the design basis sections of this report.

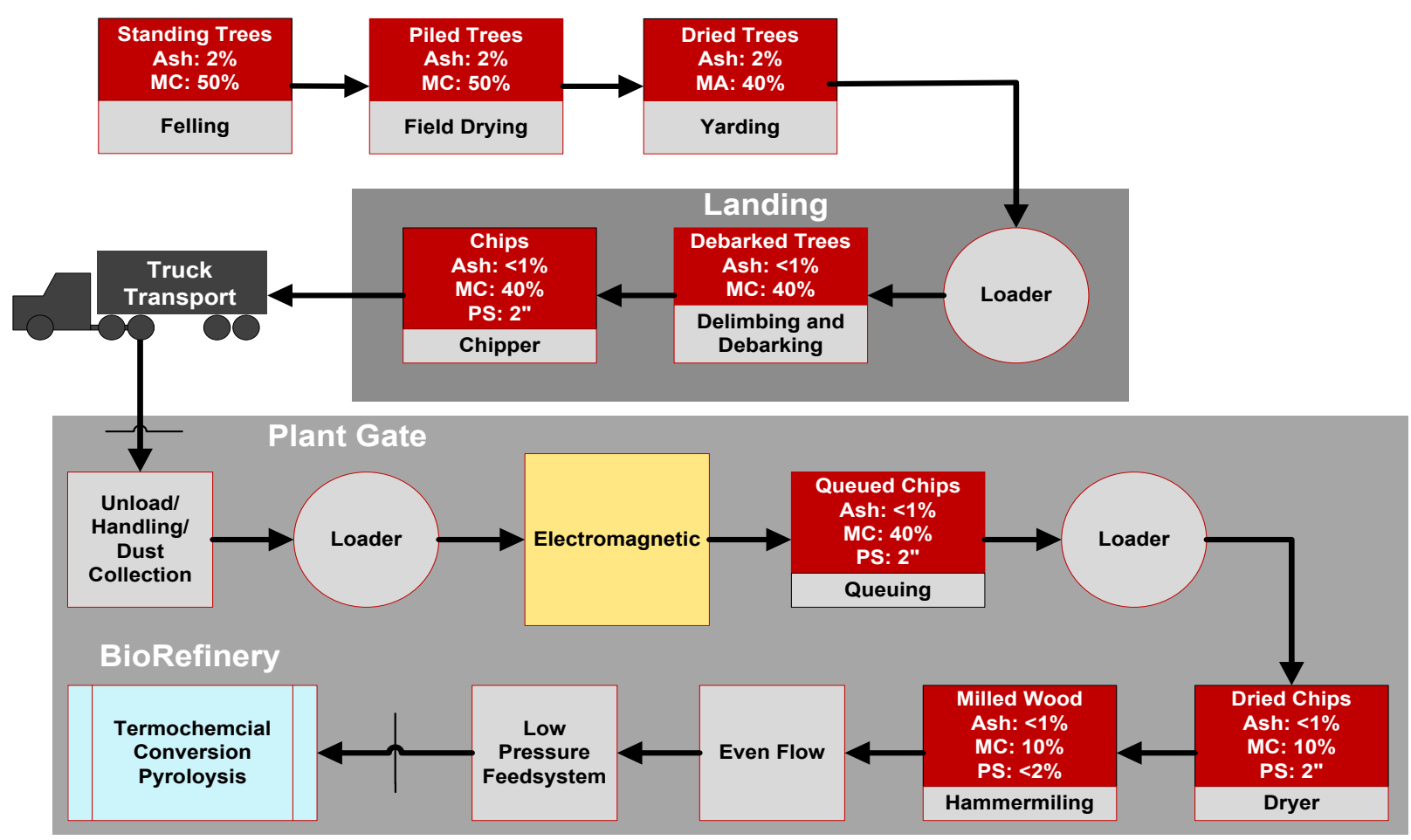

Figure 3. Flow diagram of the 2012 Relocated (Baseline) Design Case to supply thermochemical conversion refineries ${ }^{12}$.

The cost estimate of the 2013 SOT supply system (Table 4) shows a logistics costs total of $\$ 77.90 /$ dry T, compared to $\$ 55 /$ dry T for 2012 Conventional Design. Increased costs of the baseline system are attributed to the following:

- Cost escalation from $2007 \$$ to $2011 \$$ cost year 
- Lower resource yields ( $90 \%$ to $67 \%$ ), which increases harvest, collection and transportation costs

- Switch from waste heat dryer to natural gas dryer due to the separation of the depot from the conversion facility which eliminates the ability to make use of waste heat.

- Increased grower payment from the $\$ 15.70 /$ dry $\mathrm{T}$ to $\$ 25.00 /$ dry T based on the BT2 data ${ }^{5}$.

Escalation was estimated using the Chemical Engineering Plant Cost Index and Producer Price Index from 2007 to 2011. The Chemical Engineering Index is published in each issue of Chemical Engineering (www.che.com/pci). The Producer Price Index includes commercial and industrial machinery and equipment repair and maintenance costs (www.bls.gov/ppi/ppinaics811310.htm).

Table 4. 2013 SOT cost estimate (all costs are in 2011 USD).

\begin{tabular}{|c|c|c|c|}
\hline Cost Element & $\begin{array}{r}\text { Cost by Operation } \\
(\$ / \text { dry } T)\end{array}$ & $\begin{array}{r}\text { Cumulative Cost } \\
(\$ / \text { dry T) }\end{array}$ & Report Section \\
\hline Farm Gate Price & 25.00 & 25.00 & Section 4.1 \\
\hline Harvest and Collection & 22.20 & 47.20 & Section 6.1 \\
\hline Landing Preprocessing & & & Section 6.3 \\
\hline Debark/Delimb & 6.10 & 53.30 & \\
\hline Size Reduction & 6.10 & 59.40 & \\
\hline Storage & 3.80 & 63.20 & Section 6.2 \\
\hline Preprocessing & & & Section 6.3 \\
\hline Size Reduction & 5.40 & 68.60 & Section 6.3 .1 \\
\hline Drying & 17.20 & 85.80 & Section 6.3 .2 \\
\hline $\begin{array}{c}\text { Dust collection and } \\
\text { miscellaneous equipment }\end{array}$ & 0.80 & 86.60 & \\
\hline Handling & 1.50 & 88.10 & Section 6.5 \\
\hline Transportation & 14.80 & 102.90 & Section 6.4 \\
\hline Ash Dockage & 0.00 & 0.00 & $\begin{array}{c}\text { Section } \\
\text { Appendix C }\end{array}$ \\
\hline Total Delivered Feedstock Cost & & 102.90 & \\
\hline \multicolumn{4}{|c|}{ Delivered Feedstock Specifications } \\
\hline Ash Content & $<1 \%$ & & \\
\hline Moisture Content & $9 \%$ & & \\
\hline
\end{tabular}




\section{Approach of the 2017 Design Case}

The 2013 SOT Design presented above illustrates the barriers of the conventional biomass supply system that will limit expansion of a national biorefinery industry. This section will address three specific challenges for reducing the current estimated feedstock costs to achieve the $\$ 80$ /dry $\mathrm{T}$ cost target. These challenges include price of biomass resources (grower payment), feedstock quality, and the ability of the logistics system to handle increased volumes at reduced costs. First, cumulative grower payment (access costs) must be reduced. This does not suggest that the per producer payment will decrease; rather it will be shown that the grower payments can be reduced by selecting multiple feedstocks, thereby decreasing the total amount of any single feedstock, thus working lower on the supply curve and reducing the access cost. Second, the conventional biomass supply system has no mechanisms for preserving or addressing feedstock quality. Biorefinery conversion efficiency is tightly coupled to the quality of the feedstock ${ }^{6}$. There are operations that can be included in the feedstock logistic supply system that address these quality issues and deliver a feedstock that meets the in-feed quality specifications. Third, technological improvements in all supply chain unit operations must occur to reduce logistics costs and handle larger more dispersed volumes of biomass material. This section discusses the general approach of the 2017 Design Case for addressing these challenges.

\subsection{Addressing the Farm Gate Price Challenge}

The Billion Ton Update (BT2) ${ }^{5}$, which is the definitive source of national biomass supply/cost data, represents biomass access costs in terms of "farm gate" price, which includes the cost of production, harvest, collection and chipping at the landing, compensation for soil nutrient removal, and grower profit. However, feedstock logistics designs also consider harvest, collection, and chipping operations within logistics costs, therefore it is necessary to subtract harvest, collection, and landing preprocessing costs from the reported farm gate price and refer to difference as the grower payment. The grower payment would then consist of feedstock production, soil nutrient replacement, and profit margin.

Neither grower payment nor farm gate prices are constant; rather they are functions of the marginal cost of procuring the next additional quantity of biomass from that area. BT2 scenarios provide projected farm gate prices for each county in the United States for all available feedstocks for the years 2012 through 2030. When examines the BT2 results, very little biomass is accessible until farm gate prices reach $\$ 40 /$ dry $\mathrm{T}$. This means that producers are not willing to allow access to their biomass until they are offered at least $\$ 40 /$ dry $\mathrm{T}$ farm gate price.

Figure 4 below shows the step-wise supply curve for marginal and average feedstock costs versus supply quantities accessible from the BT2 scenario. These cost curves are used to determine the quantity of biomass that can be accessed at the different farm gate prices. The BT2 data is generated in increments of $\$ 10 / T$ cost targets, hence the step functions. It is important to note the difference in marginal versus average costs. The width of the boxes displays the increased amount in total tons that shows up for each type of biomass as the price increases, these incremental costs represent the marginal costs. Looking at Figure 4, at \$50/dry T around 40 million dry $\mathrm{T}$ of woody residues could be procured and a small amount of dedicated feedstocks. At $\$ 60 /$ dry T, an additional 160 million dry T of stover could be procured and an additional 15 
million dry $\mathrm{T}$ of dedicated feedstocks. The average price is the weighted average of the amount of biomass at $\$ 50 /$ dry $T$ with the amount of biomass at $\$ 60 /$ dry $T$. Note that the biorefinery would not pay the marginal cost of feedstock but the average cost.

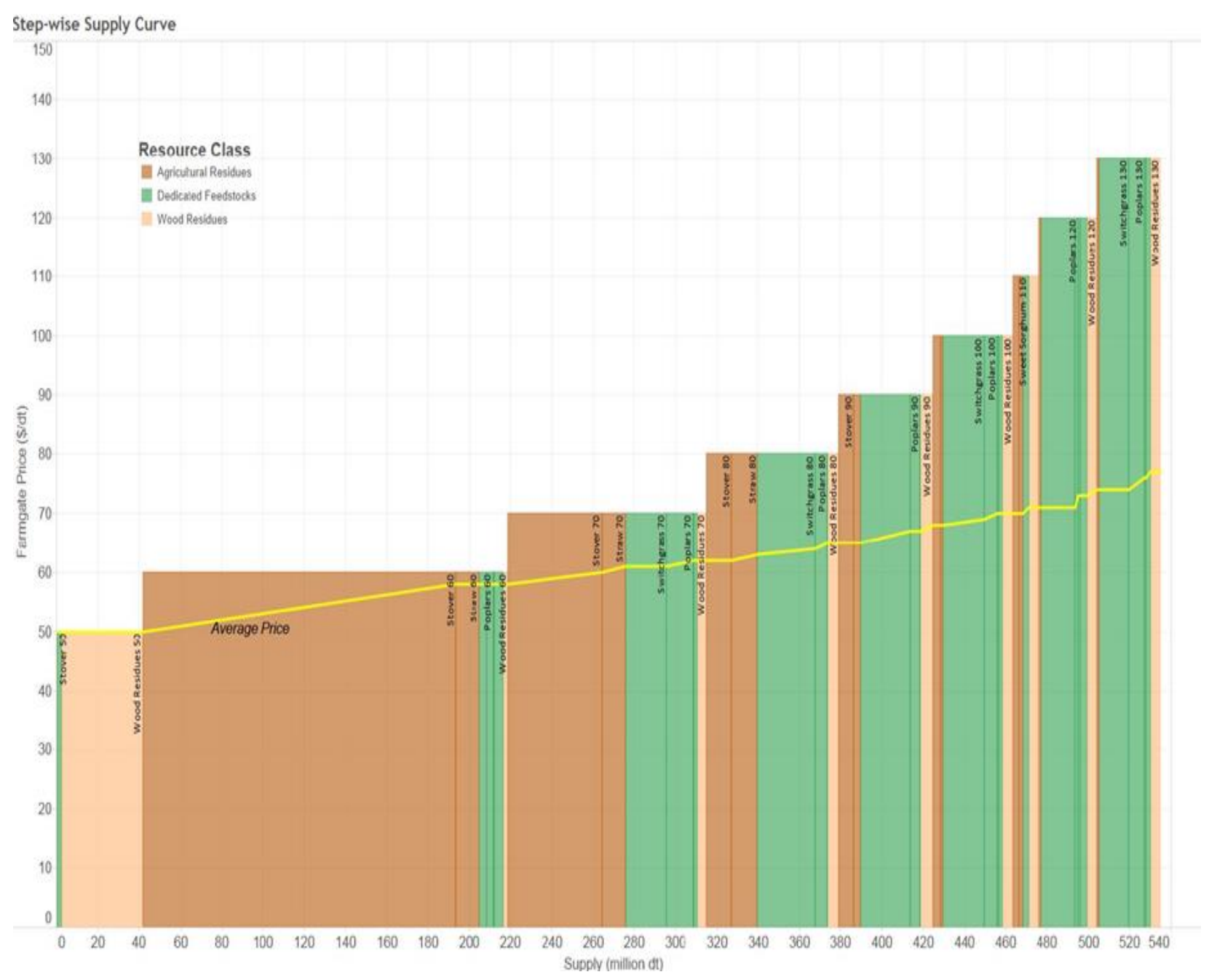

Figure 4. Marginal and average farm gate costs versus supply quantities derived from BT2 data for the U.S. predicted out to $2022^{1}$.

Figure 5 below shows a national set of farm gate prices for the U.S. At $\$ 55 /$ dry T over $100 \mathrm{MM}$ dry T of woody biomass can be secured, but only about $15 \mathrm{MM}$ dry T of poplar. If $100 \mathrm{MM}$ dry $\mathrm{T}$ of biomass was required to supply a biofuel industry, one path would be to pay an average of $\$ 55 /$ dry T for $100 \mathrm{MM}$ dry T of woody biomass. A blended strategy (i.e. blended feedstocks) could select $85 \mathrm{MM}$ dry $\mathrm{T}$ of woody biomass at $\$ 50 /$ dry $\mathrm{T}$ and supplement the rest with a combination of wheatstraw, sweet sorghum, and poplar at $\$ 50 /$ dry $\mathrm{T}$ for an average price $\$ 50 /$ dry $\mathrm{T}$. This would decrease the overall cost of biomass by $\$ 5 / \mathrm{dry} \mathrm{T}$. What still needs to be determined is if blended feedstocks will behave like a single feedstock in a conversion facility. The testing of blends is currently underway for several conversion technologies to determine feedstock behavior from front-end through finished blendstock. 


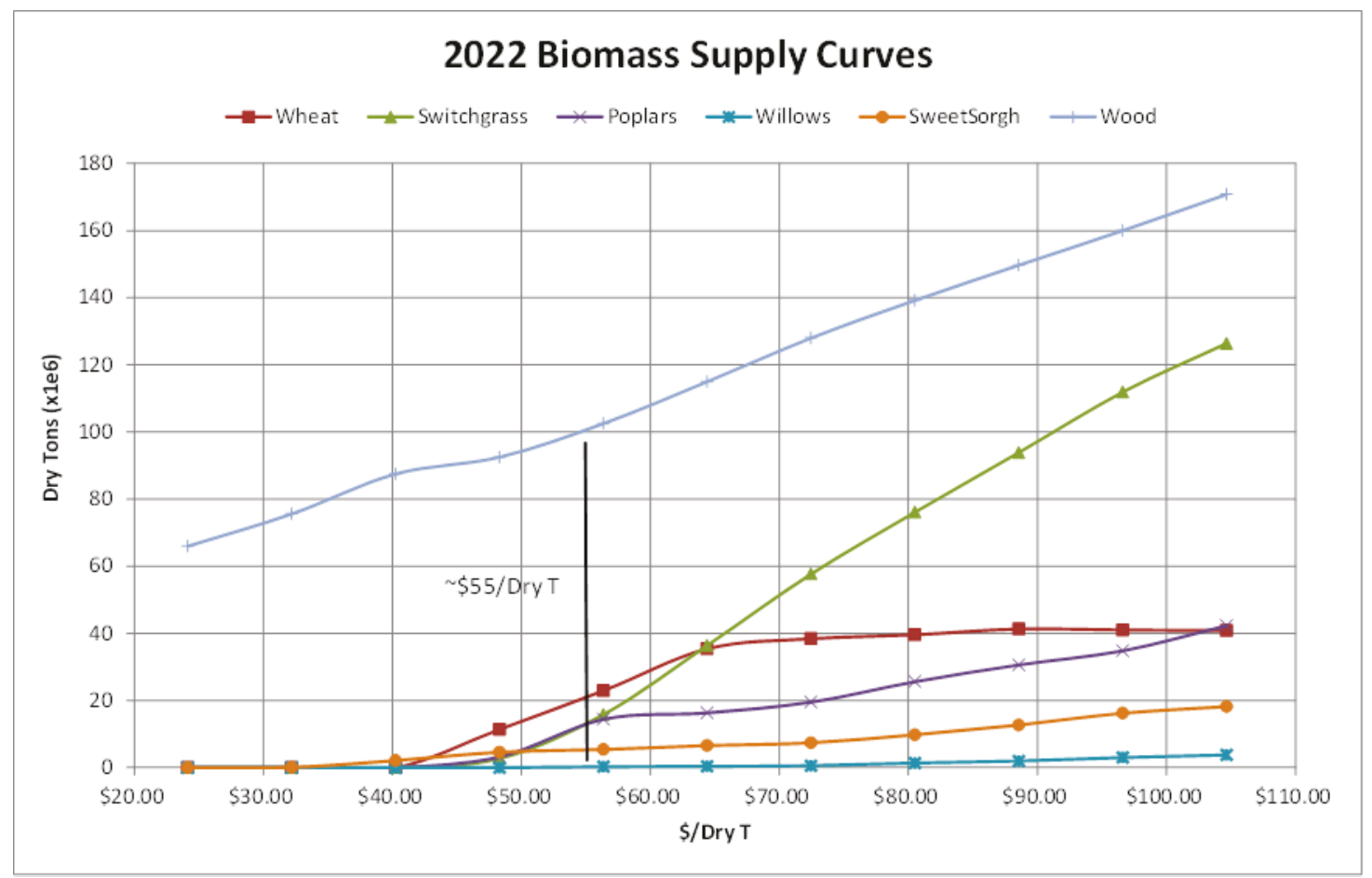

Figure 5. U.S. Marginal farm gate prices projected for 2022 by POLYSYS ${ }^{1}$.

Note: In this supply, Poplars is considered pulpwood, Wood is considered woody residues.

The 2017 Design Case will implement the multi-feedstock approach via a blended feedstock strategy. In this strategy, referred to at the least cost formulation (LCF), multiple feedstocks are blended together in specific ratios determined by availability, access costs (grower payment), and composition. The specific blendstocks chosen are conversion pathway specific based on in-feed specifications and are discussed in the conversion chapters later in this report. For further discussion on Least Cost Formulation see Appendix B.

\subsection{Addressing the Feedstock Specification Challenge}

As previously stated, compliance with biorefinery in-feed quality specification has a significant impact on bio-oil yield. Using multiple types of feedstock provides an opportunity to adjust feedstock quality; given the right blendstocks, it may be possible to blend to meet a specific biorefinery in-feed specification; thereby, reducing the need to implement a more aggressive quality control system or purchasing only the biomass that can meet the quality specifications. Blending for such purposes is a common practice in many industries. For example, blending grain to adjust quality is standard practice in the U.S. grain industry ${ }^{13}$. Similarly, different grades of coal are blended to achieve compliance with regulations regarding sulfur and nitrogen emissions in the power generation industry ${ }^{14}{ }^{15}$. Furthermore, the animal feed industry uses a range of feedstocks blended together to meet the specific nutrient requirements of the target animal ${ }^{16}$. Finally, relatively high-ash content biomass sources are mixed with low-ash coal to allow their economical use in co-fired biopower generation ${ }^{17}$. By using the blended feedstock strategy, it may be possible to develop feedstocks that conform to a desired moisture, and/or ash 
specification without using expensive preprocessing technologies. More research is required to understand the behavior blended feedstocks will have on overall fuel conversion. Even though it may be possible to blend to specification as measured by composition and physical properties, an additional challenge of the blended feedstock approach is to have the blended feedstock actually perform as well as or better than a singular feedstock in the conversion process. Better understanding of the interactions of blendstocks in the conversion process will require additional research and development focus to better inform blended feedstock development.

\subsection{Addressing the Logistics Challenge}

Moving beyond high yield areas reduces the quantity of feedstock that can be passively eliminated if not compliant with feedstock specifications. In other words, in Iowa, with high yields, only dry, low ash material can be secured while leaving the remainder of the residues for other uses. In Kansas or other lower yield areas, there may not be enough extra biomass to allow such down selection of material. The high logistics costs of the baseline case, compared to the 2012 Conventional Design Case, are mostly attributed to resource dispersion and moisture mitigation.

Additionally, the elimination of waste heat in the baseline case more realistically reflects the costs incurred by the system, since it is unreasonable to assume that the conversion facility and the depot will be co-located where they can take advantage of the waste heat. Therefore, improvements in supply systems are needed to reduce the sensitivity of grinding and transportation to biomass moisture and ash content. Additional quality specifications currently not addressed in these designs such as, high heating value (HHV) and low heating value (LHV), could also become important in future design cases. Solutions to these barriers could include fractional milling and high moisture densification with a cross flow pellet drying system. Finally, as multiple feedstocks are incorporated into the system, logistic solutions also are needed to reduce the cost that would occur due to the complexity of handling multiple types of biomass.

The blended feedstock strategy, which relies on the availability of multiple feedstock sources within an economical supply radius, adds an additional logistics challenge to the 2017 Design Case. The complex nature of this approach could bring in more business management overhead to simultaneously manage multiple feedstocks. However, this approach could increase the total amount of biomass in an area that would traditionally not have enough of a single resource to economically supply a biorefinery. Overcoming the logistics challenge of a blended feedstock design will require system-level solutions. The 2017 Design Case explores two approaches: (1) an agronomic solution based on integrated landscape management, and (2) a logistics solution based on biomass depots.

Compared to traditional cropping systems that manage productivity and environmental sustainability on an overall average field scale, integrated landscape management considers subfield-scale variability to substitute low productive crops with annual or perennial biomass crops (herbaceous or wood) for improved environmental and productive performance. For example, with the integrated landscape management approach, perennial energy crops may be planted in a pulpwood stand to improve biodiversity or protect sensitive waterways prone to erosion. Similarly, areas of a field that typically under-produce and result in lost revenue for the producer may be planted in a biomass crop (such as switchgrass) that is better suited to the 
productive potential of the soil. This approach would result in a landscape mosaic where a stand is interspersed with areas of switchgrass and willow. Successful integrated landscape management will produce both economic and environmental benefits to growers, thereby improving the biomass supply-demand dynamic and making more biomass available at lower access costs. Further, such a system alleviates the logistics challenge of dispersed resources by co-locating crops and making more biomass available within smaller supply radii than even the single feedstock scenario.

Biomass depots also may provide logistics solutions for sourcing multiple biomass resources to a biorefinery, whether these resources are largely dispersed or co-located. In this scenario, regional biomass depots may emerge as business elements to lessen the complexity of a blended feedstock supply system. The economic advantage of a depot, in this scenario, may be its specialization to supply and preprocess a single blendstock. This specialization eliminates the need for a single entity to make the capital investment and establish the expertise to contract, preprocess, and supply a diversity of resources that may have different preprocessing requirements. 


\section{2017 Feedstock Supply System Design}

The least-cost formulation (blending) approach to resource selection was introduced in Section 4.1 as a solution to the Farm Gate Price challenge (i.e., to reduce feedstock access costs). This section builds on the 2013 SOT thermochemical conversion scenario located in eastern South Carolina to illustrate the least-cost formulation approach to resource selection for the 2017 Design Case. Note: A similar approach is done for the biochemical conversion pathways based on herbaceous residues and energy crops. This approach challenges the single-feedstock paradigm by allowing available resources to compete based on cost, quantity, and quality considerations. It is also shown that such an approach can contribute significant cost reductions to biomass feedstock supply.

\subsection{State of Technology}

Most cellulosic biomass supply systems are designed around a single feedstock, typically clean woodchips for thermochemical conversion processes like fast pyrolysis. Note that these supply curves represent the projected cost (i.e., farm gate) and quantity available in 2017 based on data from the BT2 analysis. Figure 7 shows the estimated supply curve for pulpwood based on the stumpage price the buyer is willing to pay. Note that if the biofuel industry starts to require large amounts of pulpwood then the price of pulpwood will respond according to an increased demand. The price of pulpwood will increase substantially for large quantities to move into the system. The 2013 SOT Design was developed around the concept of a single biorefinery and the supply system was designed accordingly. The 2017 Design Case is designed around supplying biomass to a developing industry and not for a single biorefinery.

The woody biomass scenarios/information in the BT2 database was developed using a different set of algorithms and models than the herbaceous biomass. The usage of the data needs to be adjusted accordingly. The woody scenarios were generated from estimates from the U.S. Forest Service Forest Inventory Model and represents estimates of large areas and not county level estimates as is the case for herbaceous biomass. Due to this difference, for estimates of grower payments for small demands ( $<1$ Million dry tons), it is assumed that the current market price would be more appropriate to reflect local supply costs. Figure 6 shows the historical stumpage pulpwood prices for the southern U.S. The prices reflect approximately 57 million tons of pulpwood purchased each year. The stumpage price is for green biomass (as opposed to dry). If you assume around 50\% moisture for pulpwood, the grower payment would be double the stumpage price and average around $\$ 20 /$ dry T. A single biorefinery would require less than a million tons of biomass each year and hence would not significantly influence the stumpage price currently being paid. A single biorefinery could expect to pay around $\$ 20-\$ 25 /$ dry T when competition for the biomass is low. For the 2017 Design Case we assumed the high end for a single refinery of $\$ 25 /$ dry $\mathrm{T}$, assuming that they will be competing for the biomass and thus driving the prices towards the high end. 


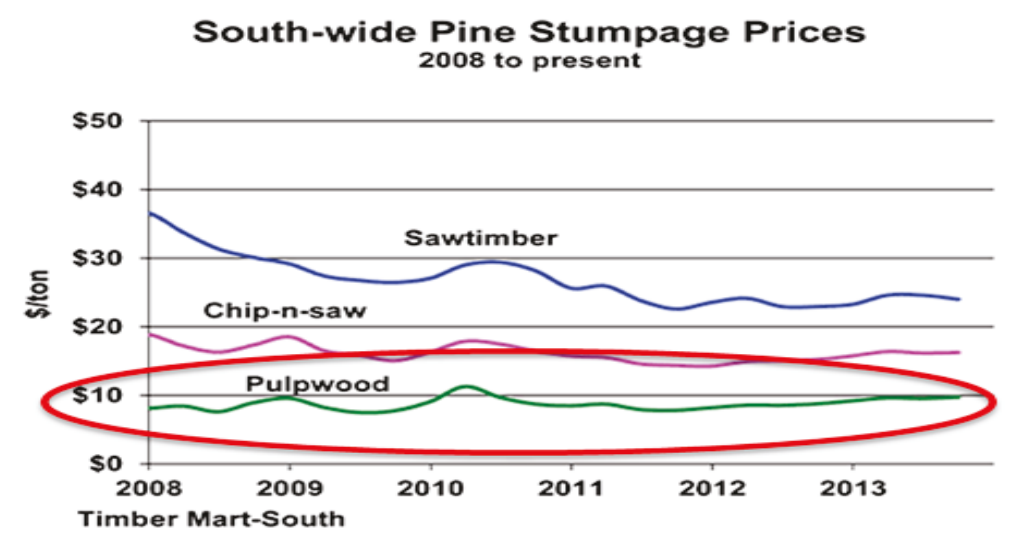

Figure 6. Historical pulpwood stumpage prices for the southern U.S. in \$/dry Ton (http://www.timbermart-south.com/prices.html).

There is a significant difference in the requested amount of biomass that will be required to fuel an industry versus a single biorefinery. Oak Ridge National Laboratory in their Billion Ton Update $^{5}$ estimated that upwards of 35 million dry $\mathrm{T}$ of woody biomass will be required for the thermochemical biofuel conversion industry as it matures toward the 2022 Design Cases. Based on Figure 7, the farm gate price to access 35 million tons would be around $\$ 50 /$ dry T. It should be noted that this is based on 2022 estimates, there may in fact not be a demand as high as 35 million tons, but it is important to analyze the system based on projected industry demand and identify potential barriers that will surface when analyzing a single biorefinery supply system. What is important is to identify that as the biofuel industry grows it will begin to require large volumes of biomass that will in fact influence the supply dynamics and increase the cost to access the biomass. If the industry does grow significantly in the future then the farm gate price will increase and could be barrier that needs to be addressed.

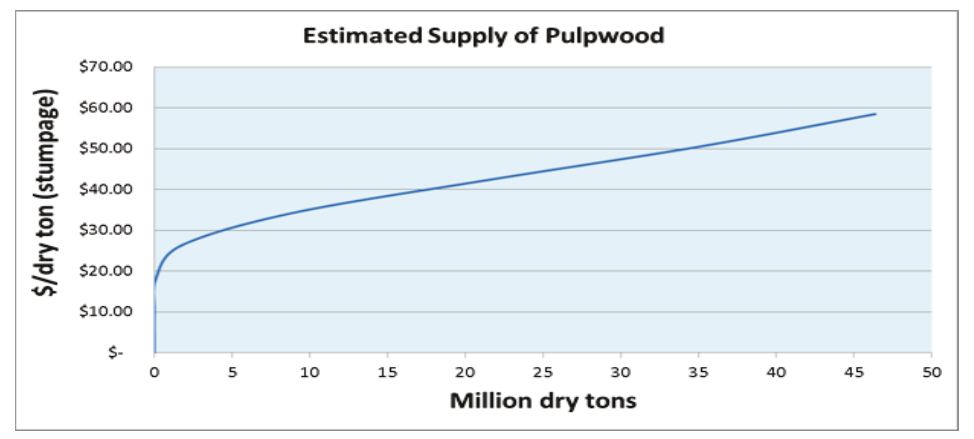

Figure 7. National estimated pulpwood stumpage prices ${ }^{1}$.

\subsection{Resource Selection Cost Estimation}

In order to represent the cost impact of the least-cost formulation approach for resource selection, it is necessary to discuss resource costs in terms of access cost, often referred to as 
grower payment. However, in order to avoid the misperception that with least-cost formulation in the 2017 Design Case the reduction of access cost means the growers get less, we use the term access cost.

Access costs are calculated from the grower payment cost curves shown in Figure 6, which are derived from historical prices. The 2017 Design Case basis discussion presented above provided the least-cost formulation approach for reducing access costs by accessing multiple feedstocks. With this approach, reduced quantities of each blendstock allows us to stay lower on the supply curve than if we had to supply the entire refinery with any single biomass feedstock. The impact of this approach is shown in Table 5. The 2013 State of Technology assumes a $100 \%$ supply of pulpwood of 909,100 dry $\mathrm{T}$ at an estimated $\$ 60 /$ dry $\mathrm{T}$ farm gate or a $\$ 25 /$ dry $\mathrm{T}$ access cost. In comparison, the 2017 Design Case blend of 45\% pulpwood, 32\% wood residues, 20\% C \& D waste, and 3\% switchgrass results in a weighted average feedstock cost that is nearly $15 \%$ lower than the access cost of pulpwood alone.

Table 5. Resource access cost estimate ${ }^{5}$ and INL Material Solid Waste (MSW Data)

\begin{tabular}{|c|c|c|c|c|}
\hline & \multicolumn{2}{|c|}{$\underline{2013 \mathrm{SOT}}$} & \multicolumn{2}{|c|}{2017 Target } \\
\hline & $\begin{array}{l}\text { Access Cost } \\
(2011 \text { \$/dry T) }\end{array}$ & Tons & $\begin{array}{l}\text { Access Cost } \\
(2011 \text { \$/dry T) }\end{array}$ & Tons \\
\hline Pulpwood & 25.00 & $909,100 *$ & 25.00 & $425,700^{*}$ \\
\hline Wood Residues & NA & NA & 26.35 & $412,800 * *$ \\
\hline Switchgrass & NA & NA & 19.67 & 25,800 \\
\hline C\&D & NA & NA & 8.15 & 172,000 \\
\hline Totals & 25.00 & NA & 21.90 & $1,036,300$ \\
\hline
\end{tabular}




\section{Feedstock Supply System Unit Operations}

A biomass feedstock supply system also called supply chain or logistics system is a complex organization of operations necessary to transform biomass into usable feedstock that can feed into a conversion process. The feedstock supply system encompasses all operations necessary to format and move biomass from standing at the location of production (field or forest) to the conversion reactor infeed system at the biorefinery ${ }^{20}$. The logistics of biomass harvest and collection, storage, preprocessing, handling, and transportation represent a large challenge to this industry. In this section we will discuss the different components (unit operations) of a feedstock supply system.

\subsection{Harvest and Collection Operations}

Harvest and collection operations encompass all activities required to cut and remove feedstock from the place of production to field side or landing. These operations also condition material for downstream use and can perform preliminary quality mitigation (moisture content, ash content etc.). The specific method of harvest is highly dependent on feedstock type. For example herbaceous feedstock (i.e., corn stover, switchgrass, wheatstraw, etc.) harvest systems could include a combine, rake, baler, windrower, and or forage chopper while a woody feedstock (i.e., thinnings, slash, pulpwood, etc.) harvest system could include a feller buncher, skidder, and chipper. Due to growing seasons, variations biomass harvests can occur due to environmental and crop conditions.

Collection involves moving harvested biomass to a centralized location, such as a field side stack or a landing deck. Potential collection equipment includes roadsiders, loaders, skidders, and cable systems. Like harvest, collection also only occurs during a specified window where optimal conditions can be achieved to maximize biomass quality and reduce material loss. For a herbaceous supply system, baling and collection typically occurs after some field drying. For a woody system collection typically happens at the time of harvest. However, for a woody system, there may be some cost advantages to delay collection until harvest is complete to improve collection efficiency and take advantage of field drying while an herbaceous system there may be advantages for using a single pass supply system where the biomass is baled as it is harvested.

\subsubsection{State of Technology}

Conventional harvest and collection employ multi-pass systems to process biomass. Existing multi pass collection systems for agricultural residues typically involve cutting the feedstock, raking the material into a windrow, and baling the windrowed material. For corn stover, cutting may or may not be done at the time of corn harvest, which impacts material quality and removal yields. In multi-pass operations, raking is performed to facilitate baling and improve yield. No consideration is given to the impact of raking on soil entrainment in the final baled feedstock. In single-pass corn stover baling, the stover is fed directly into a baler towed by the combine. This harvesting method eliminates soil contact and results in lower stover ash content and higher removal yields. However, it also eliminates field drying, which results in a higher initial bale moisture content. This elevated moisture presents a challenge to feedstock stability and increases dry matter loss in storage. 
The total ash content of research-grade corn stover samples has been reported to range from 0.8 to $6.6 \%$ across the Corn Belt of the Midwestern U.S. States ${ }^{21}$. Studies comparing single-pass to multi-pass harvest systems demonstrate total ash contents in the range of $5 \%$ to $10 \%$, respectively ${ }^{22}$, which shows that single-pass harvest systems have the potential to minimize soil contamination in production harvest operations. Table 6 shows the mean and range of ash contents for selected feedstocks and includes the effects of feedstock ash and soil contamination from harvest and collection operations. Harvest methods for these feedstocks were not specified; however, average corn stover ash content in Table 6 feedstocks suggests that the majority of the reported values were obtained from research-grade samples. Switchgrass ash contents range from 2.7 to $10.6 \%$, with an average of $5.8 \%{ }^{23}$. Minimum values correspond to physiological ash; therefore, for the purpose of this design, they are assumed to be the absolute minimum, practically obtainable values prior to further mechanical or chemical ash-reduction steps.

Table 6. Mean total ash values and ranges for selected lignocellulosic biomass

\begin{tabular}{|c|c|c|c|}
\hline & Feedstock & Average Ash (\%)* & Reported Range (\%) \\
\hline \multirow[t]{9}{*}{ Herbaceous } & Corn Cob & $2.9(13)$ & 1.0 to 8.8 \\
\hline & Corn Stover & $6.6(28)$ & 2.9 to 11.4 \\
\hline & Miscanthus Straw & $3.3(13)$ & 1.1 to 9.3 \\
\hline & Reed Canary Grass & $6.7(11)$ & 3.0 to 9.2 \\
\hline & Rice Straw & $17.5(22)$ & 7.6 to 25.5 \\
\hline & Sorghum Straw & $6.6(5)$ & 4.7 to 8.7 \\
\hline & Sugarcane Bagasse & $5.6(27)$ & 1.0 to 15.2 \\
\hline & Switchgrass Straw & $5.8(21)$ & 2.7 to 10.6 \\
\hline & Wheat Straw & $8.0(50)$ & 3.5 to 22.8 \\
\hline \multirow[t]{9}{*}{ Woody } & Oak Residue & $2.5(5)$ & 1.5 to 4.1 \\
\hline & Oak Wood & $0.6(11)$ & 0.2 to 1.3 \\
\hline & Pine Residue & $2.6(4)$ & 0.3 to 6.0 \\
\hline & Pine Wood & $1.0(40)$ & 0.1 to 6.0 \\
\hline & Poplar Wood & $2.1(14)$ & 0.5 to 4.3 \\
\hline & Spruce Residue & $4.3(2)$ & 2.2 to 6.4 \\
\hline & Spruce Wood & $0.8(5)$ & 0.3 to 1.5 \\
\hline & Willow Residue & $2.0(1)$ & 2.0 to 2.0 \\
\hline & Willow Wood & $1.5(18)$ & 1.0 to 2.3 \\
\hline
\end{tabular}

Research to-date has shown herbaceous feedstock ash content as being highly dependent on harvest equipment ${ }^{23}$. Traditional, multi-pass corn stover bales from Stevens County, Kansas, were found to range from 10 to $25 \%$ ash by mass (Figure 8), which represent increases in feedstock cost of $\$ 4.88$ to $\$ 20.23 /$ dry ton compared to the baseline level of 5\% ash. Feedstock replacement and ash disposal costs account for the change in value, which is on the order of $\$ 2.25 /$ dry ton for each $1 \%$ ash above the baseline of $5 \%$. 


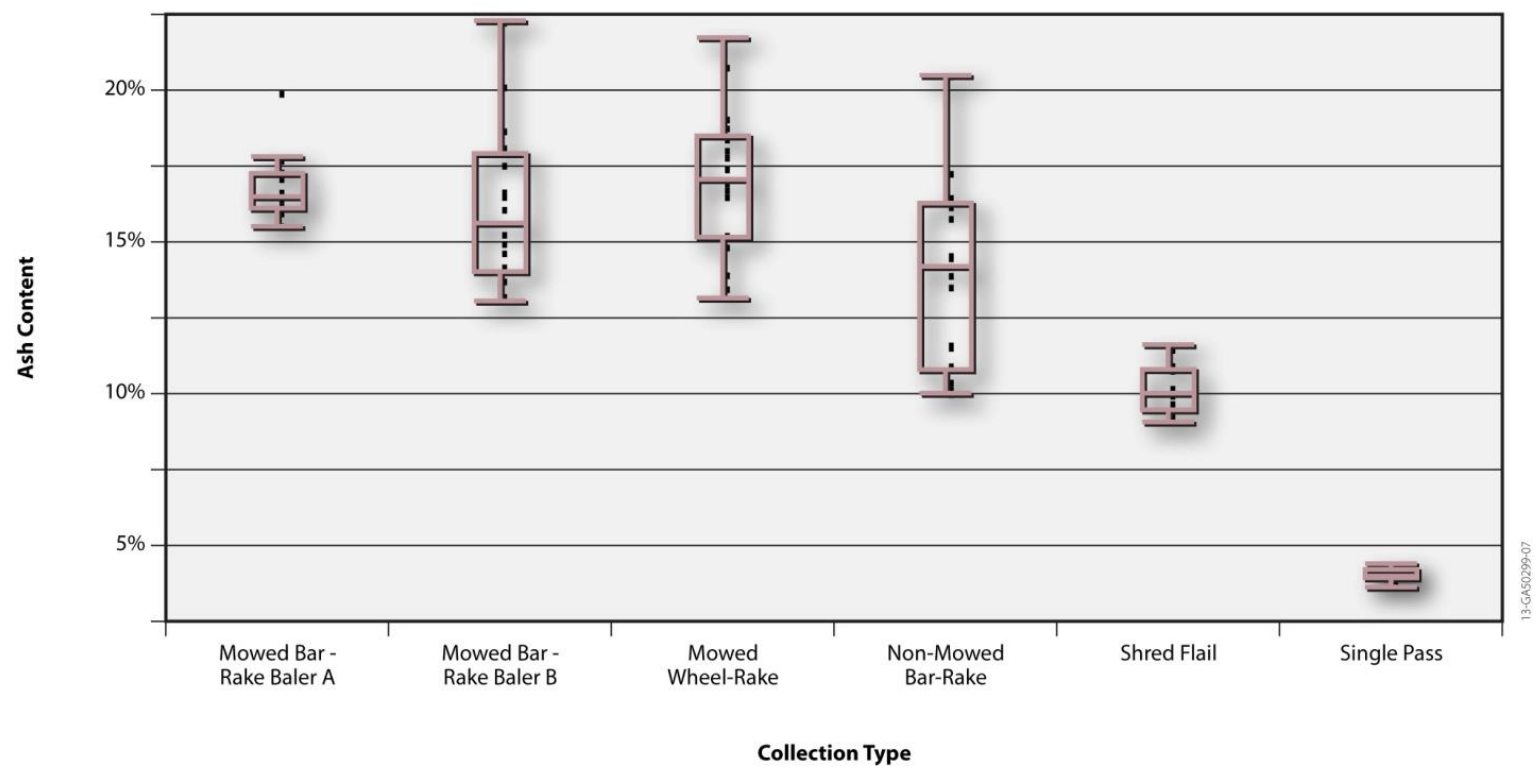

Figure 8. Ash content of corn stover bales from Stevens County, Kansas, that are collected using single pass baling and a variety of multi-pass methods, including two rakes, two balers, a mower, and a flail shredding windrower.

Single-pass bales collected from the southwest region of Kansas contained only 4\% ash (Figure 8), presenting a clear advantage to operational costs and biomass quality. Single-pass harvesting maximizes ash avoidance by preventing the biomass from contacting the soil; however, it results in increased moisture content because no in-field drying occurs. This collection method also can increase harvest yield compared to multi-pass systems, thereby decreasing the amount of acres harvested, but increasing the risk of erosion and soil carbon loss if stover removal exceeds the sustainability limits ${ }^{24}$.

In a separate study, field conditions and harvest efficiency were shown to impact corn stover bale ash content. Figure 9 shows the range of ash content measured in bales made within the same field using three different harvest methods with collection efficiencies in the range of 1 to 4 tons per acre. In this study, soil contamination was reduced through use of a flail shredder. However, for each equipment combination, ash content decreased at the expense of yield. The economic impact of yield, with the resultant increase in harvest, collection, and transportation costs, must be balanced with the need to deliver high-quality/low-ash feedstock. 


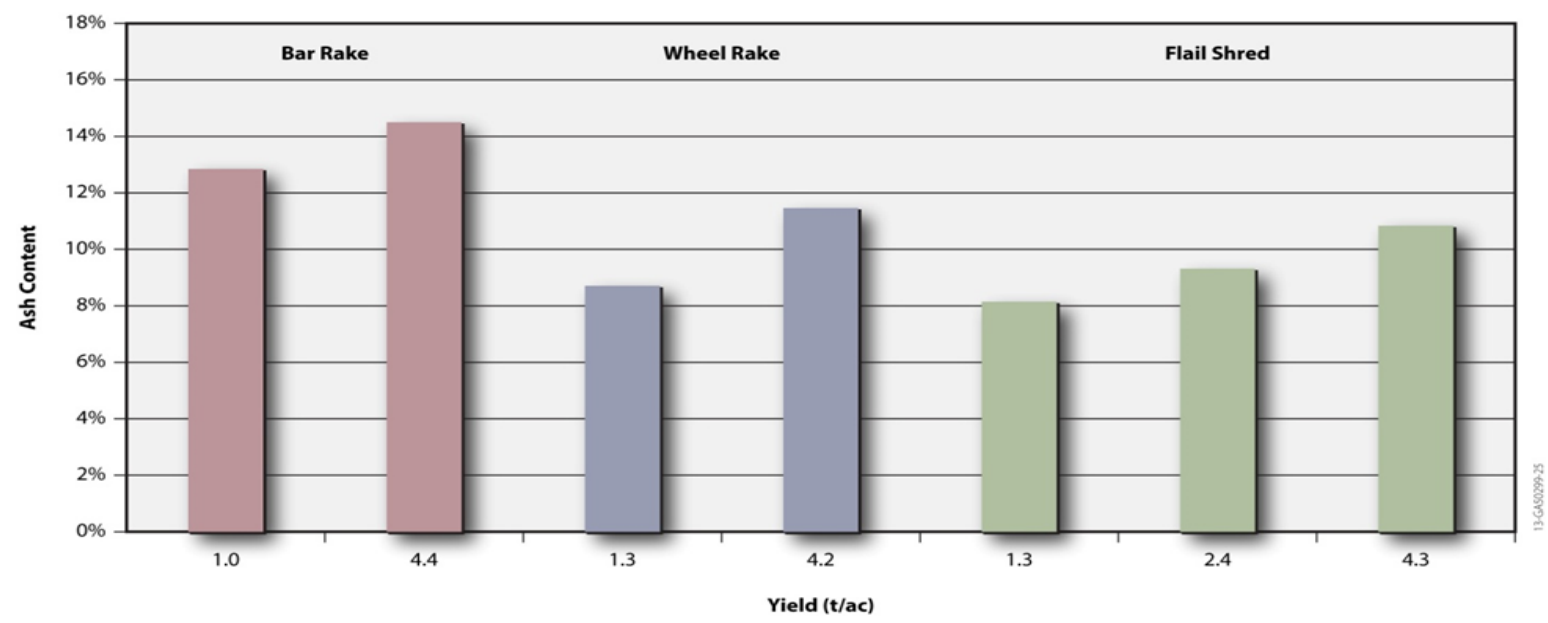

Figure 9. Ash content (bars) and yield (text) of corn stover bales from Stevens County, Kansas, show the impact of collection efficiency and windrowing equipment on yield and soil entrainment.

\subsubsection{Improvement of Harvesting and Collection}

Ongoing research addresses issues in harvesting. For example single pass harvesting systems are being developed to improve the harvesting and collecting operational efficiencies while improving the quality of material harvested by reducing induced ash from raking. A single pass system has fewer operations, less soil compaction, reduced cost and less soil contamination. Moreover, a single pass harvest and collection system targets harvesting the top portion of the plant leaving the lower plant material on the field, the harvested material has less nutrient value and more stable moisture content than the lower sections of the plant.

Harvest and collection improvement strategies for ash reduction focus on reducing soil disturbance during harvest such as reliance on mechanically driven rather than ground-driven rakes, using flail-shredding windrowers, and increasing cut height. These less aggressive collection methods may sacrifice yield for reduced soil contamination. Research is necessary to find balanced solutions that minimize cost, optimize yield, ash content, and sustainability. Further ash reduction may come from delaying harvest until after the first freeze, or by overwintering ${ }^{25}$. However, overwintering comes with the penalty of reduced yield because of leaf loss. To reduce moisture and ash contents, one option may be to focus on the upper stalk, but at a decrease in yield ${ }^{2627}$, which will have an increase transportation and handling costs ${ }^{28}$.

Harvest and collection operations typically focus on optimization of conventional equipment and processes. Optimization activities include improved fuel economy, faster harvesting, higher density balers and better collection efficiency. New research is now looking at new equipment and processes. Not all areas will reap the benefits initially from this research. For example single-pass and advanced, multi-pass harvesting systems (i.e., specialized combine operation or windrowing equipment) that reduce costs and improve ash content will likely emerge first in the highly productive regions. In less productive areas, conventional multi-pass systems will be 
operated with greater focus on reducing feedstock moisture content and improving storage stability to avoid ash enrichment throughout storage ${ }^{8}$.

\subsection{Storage}

Storage involves stockpiling material to either provide an adequate lead time to more expensive processes downstream, accumulate appropriate quantities making movement more economical, or minimize the footprint and storage infrastructure at the refinery. Storage is mainly comprised of infrastructure, which can include cement, gravel or asphalt pads, silos, storage bins, and tarps.

Additionally, storage allows feedstock preservation to enable year-round biorefinery operation from seasonally available feedstocks. Harvesting of herbaceous feedstocks, specifically agricultural residues such as corn stover and cereal straws, occurs within limited operational windows that may span weeks or months, yet ideally, conversion operations occur year-round. The goal of storage is to preserve the valuable qualities of the feedstock until they can be fully utilized within the conversion process.

Biomass is subject to degradation by fungi, yeast, and bacteria that alter the feedstock's composition through selective removal of valuable components (such as structural sugars). Consumption of these components results in dry matter loss and enrichment of other components (such as lignin and ash) within the remaining feedstock. These other components have low or no value within a sugar-based conversion process. Existing storage practices for feed and forage rely on drying (e.g., baled forage) or oxygen limitation (e.g., ensiling) to impart long-term stability. However, these operations have the potential to exceed the allowable storage and handling costs for biomass feedstocks. A more practical solution to storage losses is to control biological degradation and to maintain acceptable feedstock characteristics such as; specifications of component concentration or product yield. The relationship between feedstock properties, storage conditions, and dry matter loss forms the basis of a product shelf life, which allows perishable feedstocks to be used while they still retain their value.

Appropriate storage sites provide adequate drainage away from the stack to prevent the accumulation of moisture around the stack, provide year-round access, and preferably allow the stack to be positioned in a north-south orientation. Stacks are constructed with a bale wagon or loader and covered with high-quality hay tarps which are periodically tightened to prolong tarp life.

\subsubsection{State of Technology Herbaceous Residues/Energy Crops}

The current industry standard for assessing storage performance entirely depends on the measure of dry matter loss. While losses do occur from physical handling, such sources of shrinkage are minimized by proper practice and are not considered a major factor for improvement. On the other hand, dry matter loss from biological degradation is highly variable, difficult to measure, and difficult to control. The major factors that drive biological dry matter loss are moisture content of the material entering storage and the habitability of the biomass for microbial organisms, which includes factors such as oxygen availability, $\mathrm{pH}$, and inhibitory substances.

Conventional aerobic storage of biomass does little to limit any of these factors, because moisture contents often can be well within the range suitable for microbial growth (i.e., greater 
than 20\%) and raw biomass in a baled format presents a near-ideal environment for microbial growth and resulting degradation (e.g., ample oxygen and digestible substrate). Laboratory-scale storage experiments conducted at INL have shown significant contribution of moisture content to dry matter loss of aerobically stored corn stover, with losses ranging from as low as $6 \%$ to as high as almost $40 \%$ as moisture increases from 20 to $55 \%$, respectively (Figure 10). Although the extent of dry matter loss in these experiments matches the field-run storage trials, the loss rates are increased by a factor of approximately three because of the temperature and moisture control in the laboratory system.

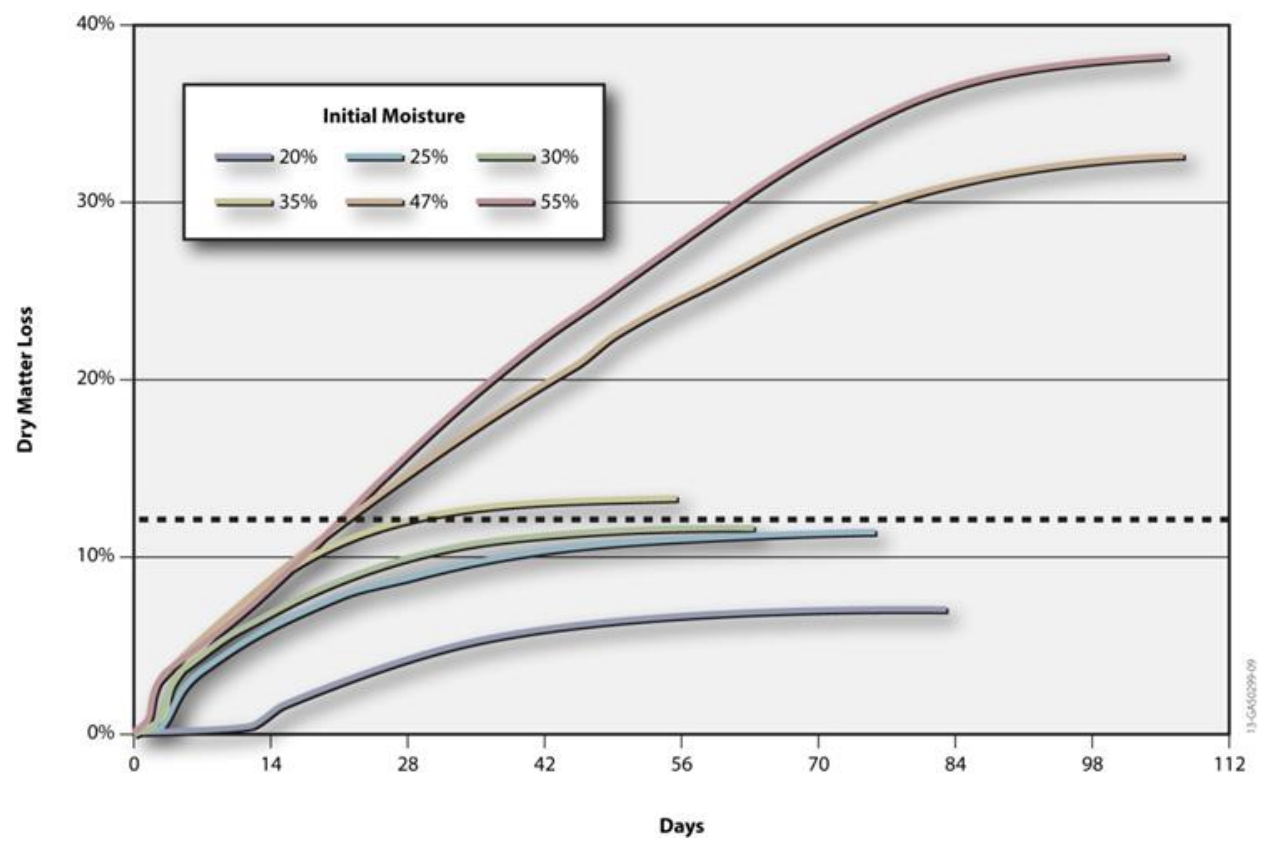

Figure 10. Dry matter loss of corn stover in laboratory storage conditions at fixed moisture contents 32.

Bale moisture tends to redistribute and even escape the stack during storage, ultimately contributing to significant moisture reduction throughout many of the bales within the stack (Figure 10). However, the end state of a stack of bales is no guarantee that its component bales did not suffer significant dry matter loss in storage or exit in a homogeneous state. The stack shown in Figure 11was placed on a gravel pad, covered with a tarp, and ultimately dried to from 30 to $19 \%$ moisture; yet it still suffered $15 \%$ dry matter loss and portions of the stack remain at high moisture. An explanation for this is supported by the data in Figure 10, which indicate that the rate of dry matter loss is highest early in storage and decreases with time and stabilizing late in storage. Therefore, unless drying occurs rather rapidly (unlike the stack in Figure 11), moisture loss during storage is not likely to reduce storage losses significantly. The ultimate conclusion is that while field drying of stacked bales does occur, the rate at which drying occurs, the extent to which material may dry, and the extent of degradation that occurred along the way is largely uncontrollable using current practices.

In addition to management of moisture and the associated dry matter loss, design considerations for biomass storage systems must include the quality of the final material. Two main 
considerations for biomass quality include the convertibility of the remaining dry matter (e.g., sugar yield from pretreatment and enzymatic hydrolysis) and the enrichment of non-convertible components (e.g., lignin, ash, and formation of inhibitors). Figure 12 shows the change in the glucan and xylan contents of the corn stover that suffered $35 \%$ dry matter loss during storage. Results show that xylan content decreased and glucan content increased in the remaining feedstock. The final compositions differ from the initial compositions, but are within the range reported for corn stover (26.5 to $37.6 \%$ glucan and 14.8 to $22.7 \%$ xylan; ${ }^{29}$ ). Notably, no clear compositional signature of dry matter loss is seen, even with significant dry matter loss. However, composition alone is not a sufficient measure of convertibility.

Field Stored Bales Harvested at 30\% Moisture \& Tarp Covered

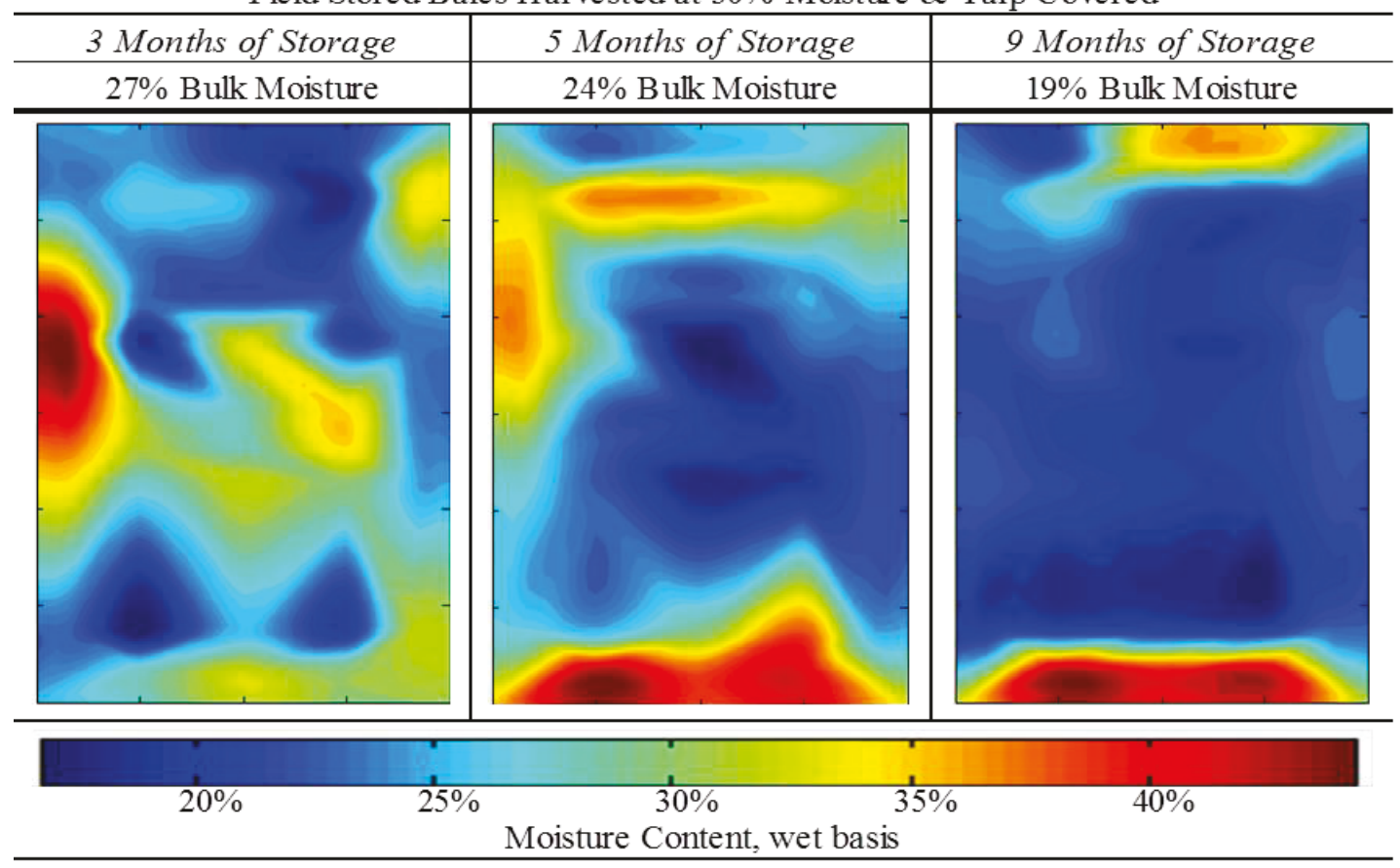

Figure 11. Change in moisture content of stacked corn stover bales in northern Iowa. Image depicts the variation in moisture content of a four-high column of bales stored outdoors for up to 9 months ${ }^{32}$. 


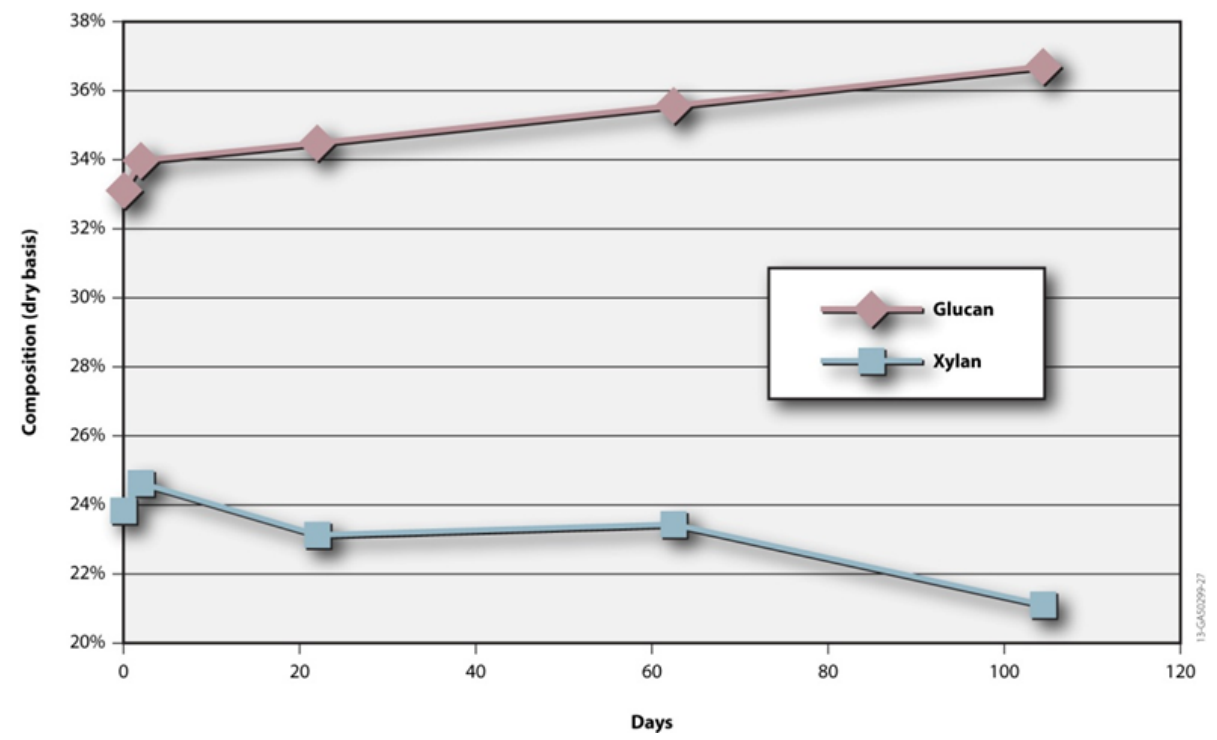

Figure 12. Change in glucan and xylan over time as corn stover is stored in laboratory reactors ${ }^{32}$.

Preliminary results suggest that losses that occur in storage result in a decreased sugar yield following pretreatment, despite this minor composition change. Storage-induced losses may occur as a result of resistance to pretreatment, over-pretreatment (conversion to furfuraldehyde and HMF in a dilute-acid pretreatment), and/or reduced enzymatic hydrolysis. Decreased sugar yields in these processes may result from the selective removal of more easily converted forms of xylan and glucan during dry matter loss. Over-pretreatment may result from the partial hydrolysis of structural sugars and formation of lower molecular weight polymers and oligomers, which are susceptible to oxidation during pretreatment. In each instance, replacement feedstock is necessary to offset the loss of available sugar in order to maintain production. Ongoing research is evaluating the impact of dry matter loss relative to the intermittent and final product yields of the remaining dry matter. Research in Fiscal Year 2015 will quantify the impacts of storage on the xylose yields during pretreatment and the glucose yields during enzymatic hydrolysis. This assumption of decreased convertibility due to degradation in storage has been applied to the 2013 SOT and is explained in more detail in the next section.

\subsubsection{State of Technology Woody Biomass}

Unlike crop residues, woody feedstocks may be harvested as needed in most southern US regions and therefore have less need for long-term storage. However, in-field storage may be used to reduce the moisture content of whole tree or delimbed piles ${ }^{30}$ and stacks ${ }^{31}$ depending upon pile configuration and local climate. The 2013 Design Case assumes 10\% reduction in moisture content in pulpwood from $50 \%$ to $40 \%$ as a result of field drying prior to preprocessing at the landing. This is a very conservative assumption and therefore the 2017 Design Case assumes a $20 \%$ reduction in moisture from $50 \%$ to $30 \%$ as a result of field drying prior to landing preprocessing. Although not used in the 2013 Design Case baseline, wood residues (limbs and tops) are assumed to have a moisture content of $40 \%$ both before and $30 \%$ after storage. 
Current SOT and 2017 Design Case rely upon available whitewood chips from pulpwood and residues (tops and limbs) preprocessed at the landing. Dry matter losses during in-field drying of residues is expected to be low; measured losses under laboratory controlled conditions at temperatures ranging from $15^{\circ}$ to $35^{\circ} \mathrm{C}$ ranged from $1 \%$ to $2 \%$ over one month ${ }^{32}$. Storage of logging residues in windrows and bundles was reported ${ }^{33}$ to result in a $<1 \%$ dry matter loss per month in storage; most losses were attributed to the loss of foliage. While the mechanical loss of small tops and limbs during chain flail debarking is reported to be $15 \%$ by mass ${ }^{34}$, all costs incurred to get the material into the debarked chip format will be attributed to the mass of the chipped biomass. In this design, the flail and trommel remove material that is out of specification for ash content and is thus not a part of the marketable portion of the biomass.

Following landing preprocessing the current design case relies upon limited on-site chip storage sufficient to supply three days of feedstock. Chip storage piles present favorable conditions for microbial growth, biological self-heating, and chip deterioration, which results in feedstock loss, quality changes, and risks to worker health and safety ${ }^{353637}$. On-site chip quantities are limited to reduce these risks. Design assumptions call for chips to be stored outdoors and handled using a front-end loader. In the 2013 baseline short-term chip storage of $40 \%$ moisture biomass is assumed to suffer $5 \%$ dry matter loss. This assumption is considered to be conservative for the timeframe in question. INL research using intermediate scale pine chip piles shows temperature increases in the range of 60 to $65^{\circ} \mathrm{C}$ within three to seven days of storage depending on location within the pile ${ }^{38}$. Extended exposure to these high-temperature conditions results in acetic acid formation and changes to color and texture of the chips ${ }^{39}$. In laboratory studies conducted by INL using fresh pine chips at 50\% initial moisture, dry matter loss in storage simulation reactors reached $1.5 \%$ by three days in storage during the initiation of self-heating, $2.5 \%$ by one week when a maximum temperature of $60^{\circ} \mathrm{C}$ was reached, and $6 \%$ by one month as shown in Figure 13. Based on these research samples, the dry matter loss assumption of $5 \%$ in the 2012 Design Case baseline does not account for longer storage lengths on-site beyond the three day window, potential pile wetting due to precipitation and/or moisture migration, and mechanical handling losses.

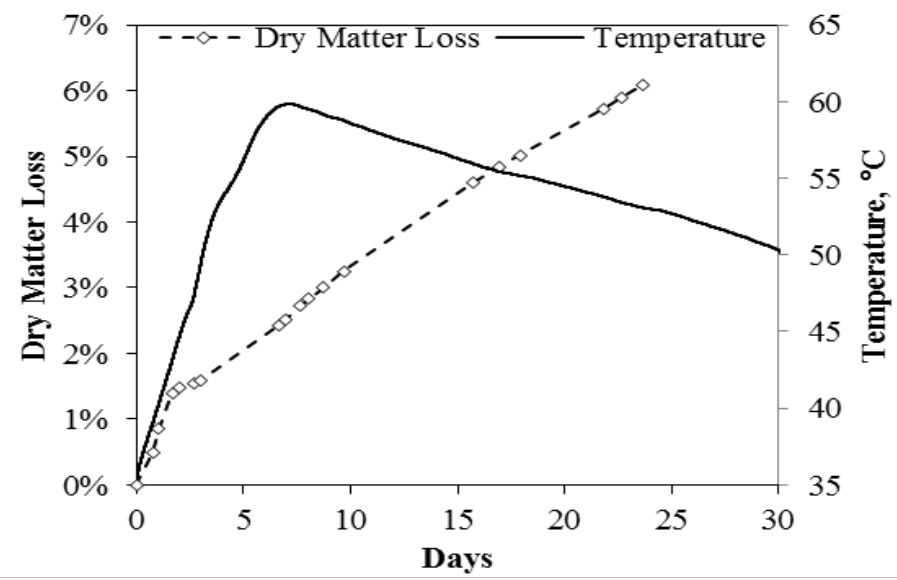

Figure 13. Dry matter loss and self-heating of 50\% initial moisture pine chips stored under aerobic conditions using laboratory scale reactors at INL. 


\subsection{Preprocessing}

Preprocessing includes any physical or chemical activity that changes the material such as: chipping, grinding, drying, and densification. Preprocessing may also include necessary auxiliary operations such as: dust collection and conveyors. In general, the goal of preprocessing is to increase the quality and uniformity of biomass in order to decrease transportation and handling costs further along the supply chain. Different types of preprocessing operations described below:

\subsubsection{Comminution}

Biomass size reduction, broadly referred to as comminution takes biomass from its as received condition (i.e., baled, log, or coarse shredded) to the final particle size specification required by the end user. Energy-intensive mechanical preprocessing operations like comminution tend to be expensive; therefore optimization of this stage of preprocessing allows opportunities to reduce the amount of equipment required and costs. Several aspects of size reduction are considered in order to optimize the system to reduce cost. Design and performance considerations include the size distribution of the final milled feedstock and the energy required to process the material. Each size reduction process encounters biomass loss which has a double cost associated with the process. First, the lost material must be accounted for by accruing additional biomass to make up for the loss. Second, all cost prior to the loss are lost so any losses late in the supply chain can have substantial economic impacts. For instance, encountering a 5\% loss of material at the biorefinery will mean that the harvest, collection, chipping and hauling costs will be lost for that portion of the material.

Size reduction, or comminution, is an essential component of biomass logistics as downstream conversion prefers a specific in-feed particle size. Additionally, size reduction aids in downstream handling and transportation by increased load density and flowability. Comminution can be conducted with either chippers or grinders but the cutting mechanisms are quite different. Chippers use knives to cut or shear material while grinders use hammers to smash or crush material. In general, the type of material dictates the type of comminution equipment to use. In particular, grinders are used for contaminated material like C\&D waste or wood residue due to their reduced sensitivity to wear compared to chippers. Size and configuration also play a role in equipment selection as chippers tend to perform better with uniform orientation of in-feed while grinders do not have an orientation preference.

Hammer mills generally are considered the current SOT for biomass comminution due to their high throughputs and versatility in processing a wide range of materials. Grinding and separation process is described below.

\subsubsection{Sequential Two-Stage Grinding}

Conventional milling operations involve two sequential size-reduction steps to arrive at the final particle size specification. The first stage of the size reduction process takes the as-received biomass and converts it (through grinding or chipping) into a product that can be further preprocessed. In the 2013 SOT scenario, the first stage size reduction is followed by drying and second-stage size reduction. The 2013 SOT configuration of the first-stage grinding/chipping process uses a 2 to 3 -in. screen for coarse size reduction. This size and type of screen provides enough size reduction for subsequent drying and final grinding. 
The role of the second-stage grinder is to reduce the particle size further in order to meet particle size distribution requirements. A typical second-stage size reduction process will use a 19 to $25-$ $\mathrm{mm}$ screen to produce a mean particle size of 2.75 to $3.25 \mathrm{~mm}$. While conventional milling processes achieve the desired mean particle size, they often have wide particle size distributions, with a large percentage of undersized particles referred to as fines.

\subsubsection{Pneumatic separation}

Pneumatic separation has been found to be effective at increasing throughput capacities of grinders by separating out the finer particles quickly so that they do not remain in the grinder where they reduce grinding efficiency particularly with low density feedstocks like biomass. Pneumatic discharge systems can increase capacity by 3 to 4 times. Without pneumatic discharge, processed material is thrown in every direction including up the infeed. The pneumatic system helps to force the processed material quickly in the right direction - through the screen ${ }^{40}$. Figure 14 shows the improvement in comminution capacity due to the addition of a pneumatic transfer system. Additionally, pneumatics can affect the quality of feedstock by removing moisture and potentially ash illustrated in Figure 15, Figure 16.

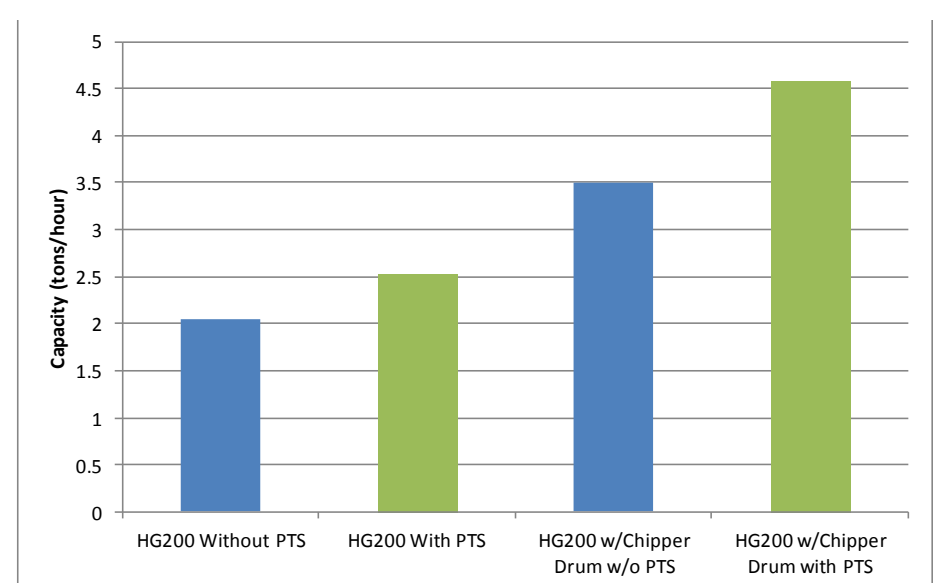

Figure 14. Comparison of comminution capacities (tons of through put per operating hour) for woody biomass as a result of adding pneumatic transfer assist (PTS) ${ }^{38}$.

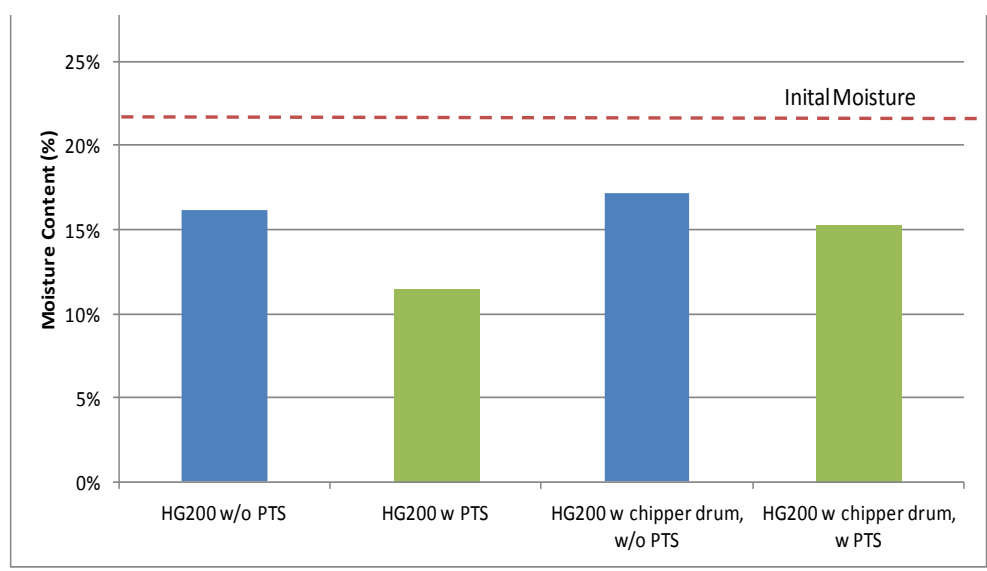

Figure 15. Change in moisture content during comminution using pneumatic transfer assist (PTS) ${ }^{38}$. 


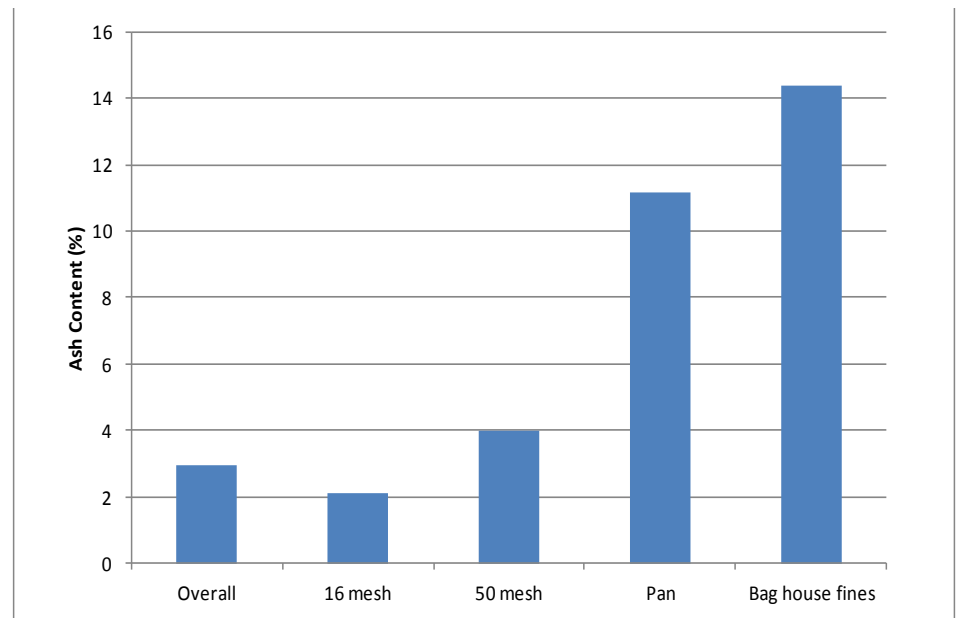

Figure 16. Ash content in various screen sizes for pinyon-juniper biomass ${ }^{38}{ }^{41}$.

\subsubsection{Fractional Milling Design Basis}

An analysis of the particle size distributions of the milled biomass after first-stage grinding, shows that much of the material already meets particle size specifications. With the conventional, two-stage grinding approach, all the material is further processed through the second-stage grinder, which results in over processing, generation of more fines, and needless consumption of additional grinding energy.

Fractional milling design solves this problem by introducing a separations step between the first and second-stage grinding operations to remove the material that already meets the size specification, thereby passing on only the remaining oversized material for further size reduction. As an example, consider the sieve analysis of the corn stover grind fractions shown in Figure 17. This chart shows the sieve fractions that result from hammer mill screen sizes ranging from 1 to 6 in. Assuming a particle size specification of $1 / 4$-in. minus (i.e., all material passing a through a $1 / 4$-in. screen), the data show that over $75 \%$ of the material processed through a 1 -in. screen and about $45 \%$ of the material processed through a 6 -in. screen can bypass the second-stage grinder. The result of this approach is a tighter particle size distribution, reduced fines, and reduced grinding energy consumption. 


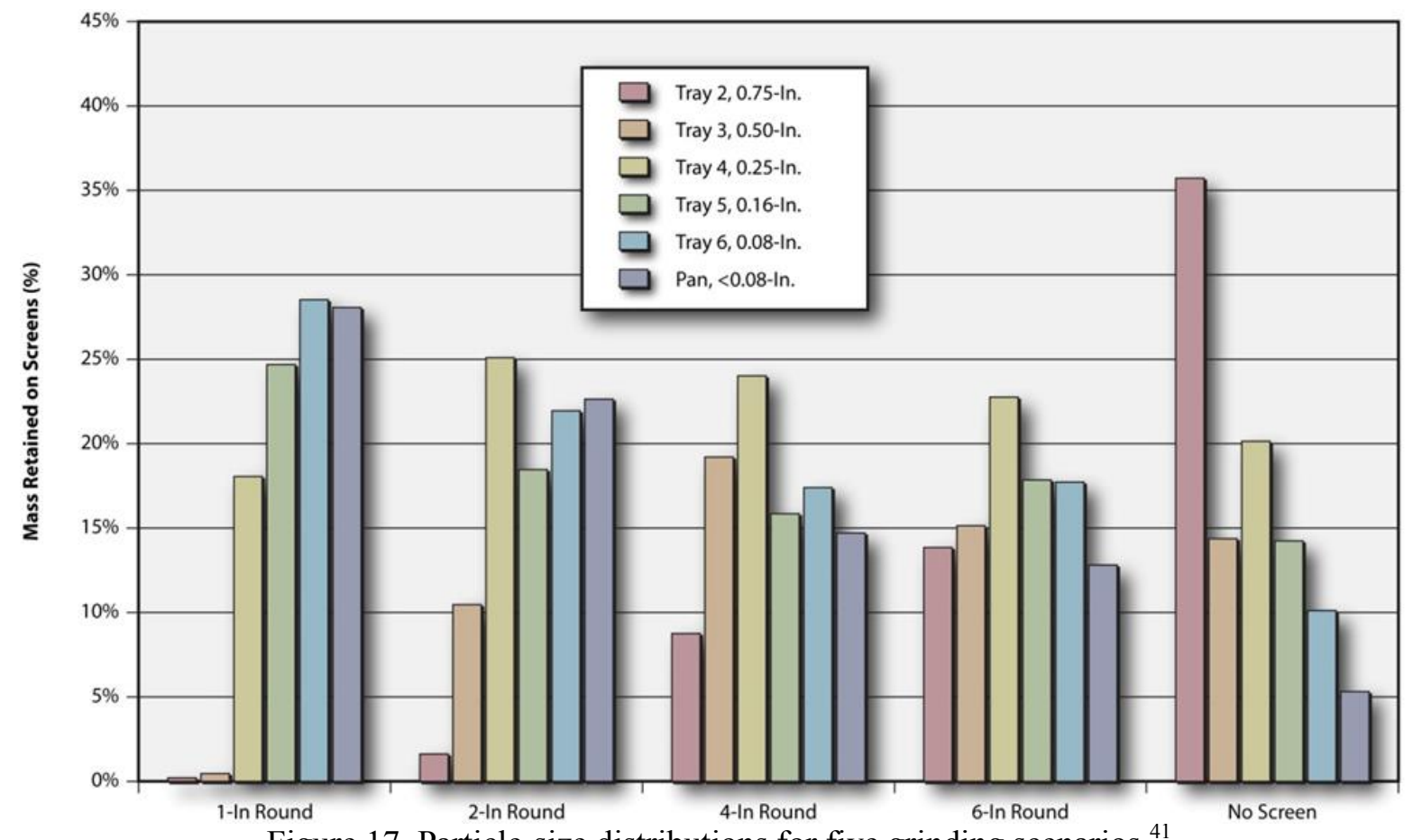

Figure 17. Particle-size distributions for five grinding scenarios ${ }^{41}$.

With conventional, two-stage milling, the choice of the screen size in the first-stage mill is based on balancing energy consumption and mass flow rates through the two operations. Figure 18 shows the specific energy consumption (i.e., total consumed power) data for milling corn stover through a combined two-stage process. In these tests, the screen size of the first-stage hammer mill grinder was varied from 3/16 to 6 in. as shown on the chart. The second-stage hammer mill grinder was configured with a 3/16-in. screen for all tests. The highest energy consumption is observed when size reducing in a single-pass through the first-stage grinder. These tests reveal that it often is very difficult to optimize a coupled, two-stage-size reduction process, because the second-stage mill often regulates the capacity of the first-stage mill. For a specific material and moisture content, the system, whose results are shown in Figure 18, was operating in "a sweet spot" (where capacities are evenly matched and grinding efficiencies are the greatest) when either a 1 or 2 -in. screen in the first-stage grinder was used. The data show that with larger firststage screen sizes, the second-stage grinder has to work harder, reducing the capacity of both itself and the upstream grinder feeding. 


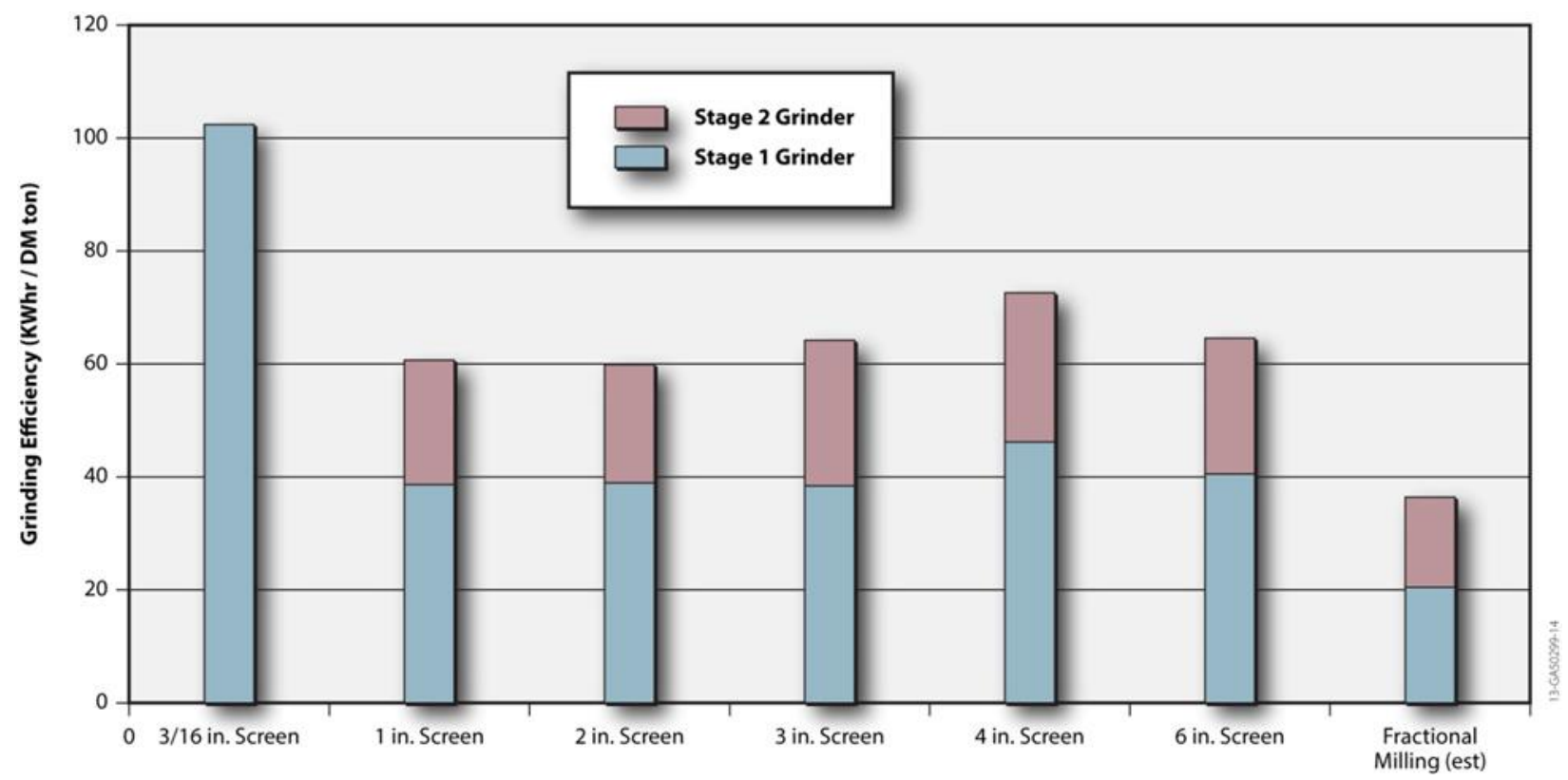

Figure 18. Comparison of conventional, two-stage grinding and fractional milling ${ }^{41}$.

Decoupling the two sequential grinding operations provides an opportunity to optimize the two systems independently. For example, when the first-stage grinder is operated alone and the throughput is not constrained by the throughput of the second-stage grinder, the specific energy consumption of the first-stage grinder is reduced substantially (Figure 18). Optimization of the first-stage grinder for the fractional milling design is accomplished by using a 6-in. screen to maximize throughput and to minimize the amount of fines produced. Extrapolation of the specific energy data shown in Figure 19 to estimate a design basis for operating with a 6-in. screen provides an estimated specific energy of $12 \mathrm{~kW}-\mathrm{hr} / \mathrm{ton}$. This is about a $70 \%$ reduction in energy compared to the current 2013 SOT. 


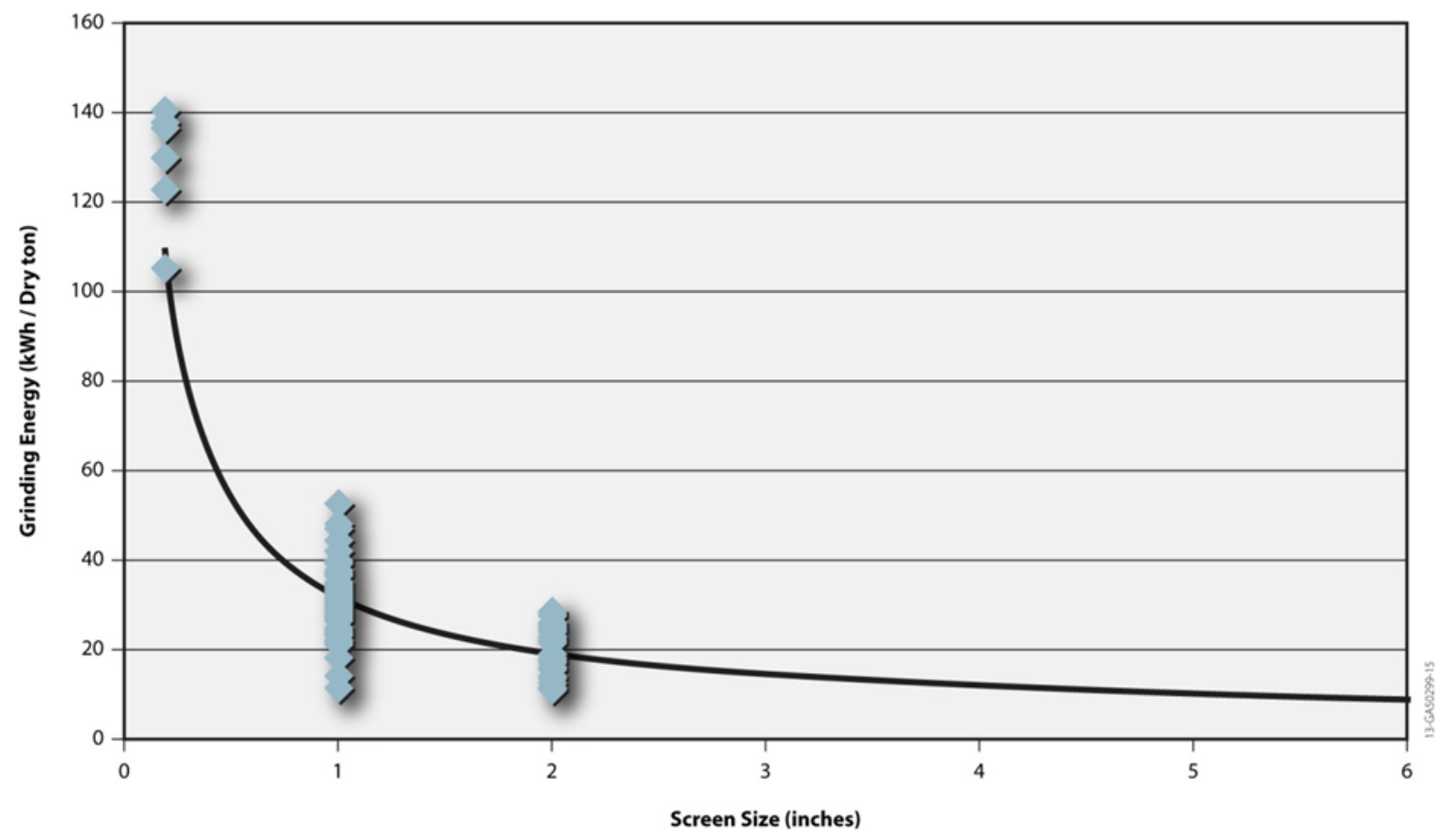

Figure 19. Grinding energy and throughput is highly dependent on screen size ${ }^{41}$.

Hammer mill systems tend to be highly sensitive to biomass moisture content, with energy consumption increasing dramatically as moisture content increases. This is illustrated in Figure 20 , which shows that the sensitivity to moisture also varies with screen size. The majority of the data used in this design to support technical targets for fractional milling was derived from hammer milling of dry (i.e., approximately 15\% moisture) biomass. Therefore, when establishing the fractional milling design basis, it is necessary to first develop the targets based on a dry biomass scenario and extrapolate using more limited data sets under higher moisture scenario. 


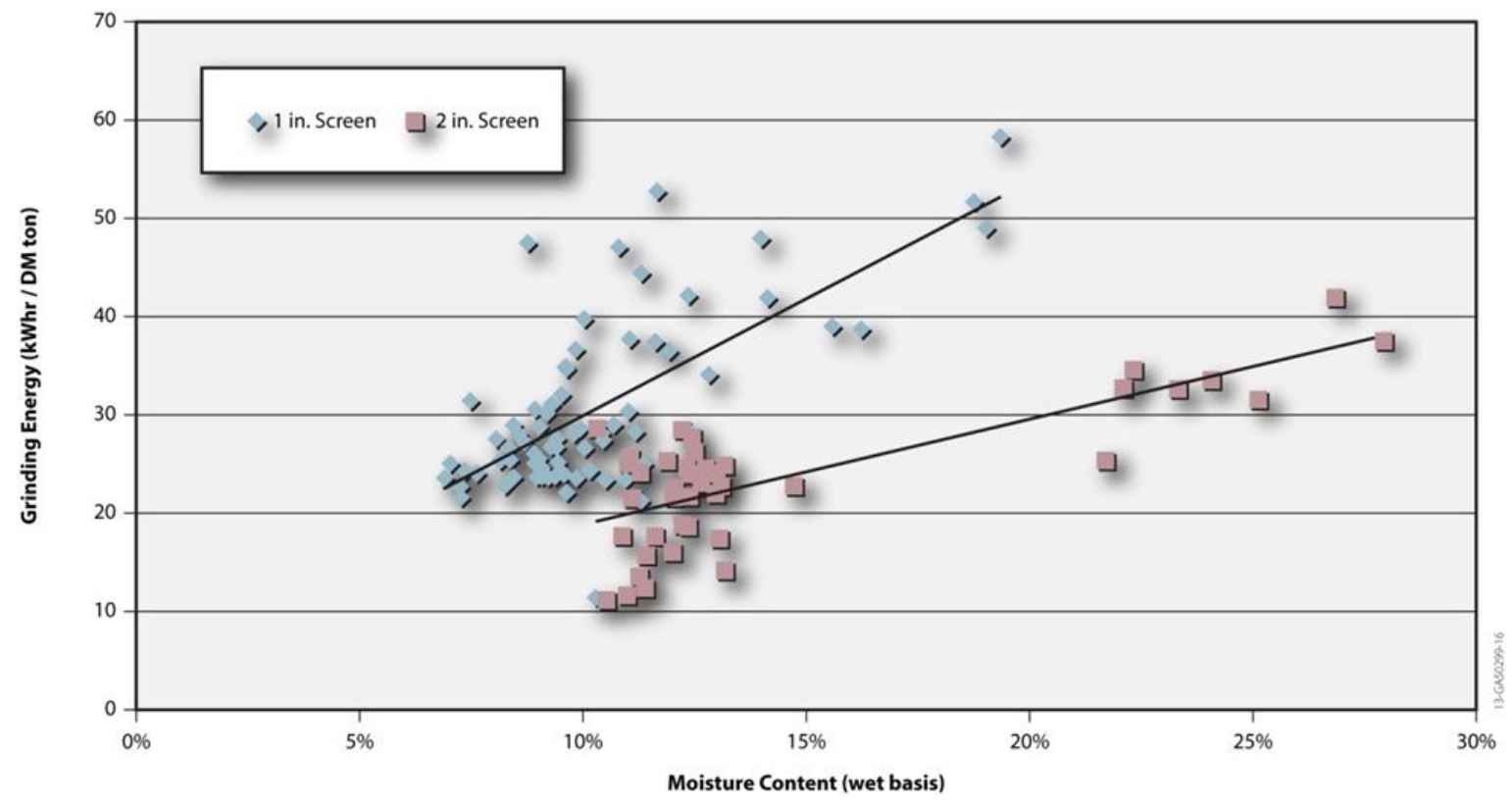

Figure 20. Hammer mill energy consumption is highly dependent on biomass moisture content (INL PDU Data).

\subsubsection{Fractional Milling Dry Biomass}

In this scenario, the data presented in Figure 18, Figure 19, and Figure 20 are used to estimate the expected performance of a fractional milling design for processing dry (i.e., approximately $15 \%$ moisture) herbaceous biomass.

First-stage size reduction: As explained above, decoupling the first and second-stage size reduction processes allows us to independently optimize the two systems. This is accomplished using a first-stage hammer mill with a 6-in. screen to maximize throughput and to reduce the amount of fines that are typically generated when using smaller screens. According to Figure 19, we estimate that the energy consumption for first-stage milling to be about $10 \mathrm{kWhr} / \mathrm{ton}$.

Second-stage size reduction: The design basis of the second-stage grinding process in this scenario assumes that the decoupled fractional milling process will allow the second-stage grinder to operate at the minimum energy requirements $(21 \mathrm{kWhr} / \mathrm{ton})$.

Separation: The fractional milling design inserts a separator between the first and second-stage comminution processes to separate the material from the first-stage comminution process that meets the size specification from those that are oversized and require further processing through second-stage comminution. Based on a 1/4-in. particle size specification, the separator will be configured with a $1 / 4$ - in. screen; therefore, only the material that is retained on the screen will be conveyed to the second-stage mill. Using the particle-size distribution data shown in Figure 17, we assume that following the first-stage hammer milling through a 6 -in. screen, approximately $45 \%$ of the material will pass through the $1 / 4$-in. separator screen, and the remaining $55 \%$ will be passed to the next mill. With only $55 \%$ of the material requiring further processing through the 
second-stage grinder, the estimated effective specific energy consumption for second-stage fractional milling is $12 \mathrm{kWhr} /$ ton $(21 \mathrm{kWhr} /$ ton multiplied by 0.55$)$.

\subsubsection{Fractional Milling High-Moisture Biomass}

Considering that the 2017 Design Case includes preprocessing of higher-moisture biomass, the design basis for fractional milling of dry biomass requires an additional adjustment of the specific energy assumptions for both the first-stage and the second-stage to account for the increased energy requirements due to moisture.

First-stage size reduction: Data for single-stage grinding (Figure 20) shows that the sensitivity of energy consumption to moisture content decreases with an increasing screen size. According to Figure 20, as moisture increases from 15 to $30 \%$, grinding-specific energy increases by 85 and $65 \%$ for 1 -in. and 2 in. screen sizes, respectively. Assuming this trend continues, a 6 -in. screen will be much less sensitive to moisture content than the 1 and 2-in. screens shown. We estimate that energy consumption with a 6 -in. screen will increase by about $50 \%$ as moisture content increases from 15 to $30 \%$. Applying this to the first-stage energy consumption assumed in the dry scenario above, the estimated specific energy consumption for first-stage hammer milling increases from $10 \mathrm{kWhr} /$ ton at $15 \%$ moisture to $15 \mathrm{kWhr} /$ ton at $30 \%$ moisture.

Second-stage size reduction: A limited INL data set indicates that the 21-kWhr/ton energy consumption measured for hammer milling corn stover at 15\% moisture (Figure 18) increases to about $60 \mathrm{kWhr} /$ ton at 30\% moisture. For the 2017 Design Case, we assert that improvements to comminution systems are achievable to reduce the sensitivity of these systems to biomass moisture content. While improvements to hammer mill systems may be achieved, shear milling technology generally is considered a better option for higher-moisture materials. A preliminary data set obtained from testing at INL of a prototype shear mill from an industry collaborator suggests that shear mill technology may be capable of reducing comminution energy requirements at higher moisture contents to the level achieved with hammer milling at the lower moisture levels. Accordingly, a technical target of $21 \mathrm{kWhr} /$ ton is established for the 2017 Design Case second-stage size reduction process (taken from the 2-in. screen data shown in Figure 20).

Separation: The separations target for the high-moisture scenario is the same as the low-moisture scenario discussed above. Achieving this target may be more difficult at higher moisture levels, because the higher-moisture material will likely be tougher and less prone to shattering than the low-moisture material. Nonetheless, $45 \%$ of the material passing through the $1 / 4$-in. separator screen is established as the target for the 2017 Design Case separation design basis. As was described for the dry fractional milling scenario, this separations target results in effective energy consumption for second-stage comminution of $15 \mathrm{kWhr} / \mathrm{dry} \mathrm{T}$ (calculated as $21 \mathrm{kWhr} / \mathrm{dry} \mathrm{T}$ times 0.55 ), the total effective energy consumption target for fractional milling is $35 \mathrm{kWhr} / \mathrm{ton}$.

\subsubsection{Drying}

Drying is important from various standpoints. Drying permits long-term storage without deterioration and aids in the production of a better quality product. Drying could occur at the 
field, biorefinery, depot, or during unit operations. Agricultural materials and therefore biomass materials are dried using a variety of procedures due to differences in material properties and desired outcomes. Factors that influence drying technologies include tolerance to temperature (i.e. materials may cook or undergo adverse physical changes), response to humidity (i.e. material undergo physiological may require specific air humidity), compression strength of material (i.e. materials may crush or deform and must be dried in thin layers) and fluidity (i.e. poor flowing material must be adjusted for proper angle of repose). Additionally quantity of materials as well as desired rate, and environmental conditions (weather) influence the technology applied.

The simplest form of drying is passive drying or field drying. This type of drying takes advantage of existing environmental conditions to reduce biomass moisture in the field or stand, but it is not consistently reliable across all spatial and temporal variations. In contrast, active drying utilizes engineering controls to stabilize material and reduce moisture. Active drying includes rotary dryers, cross flow dyers, and batch or bin dryers. Rotary dryers reduce moisture by rotating a material through a heated air in a drum at a set speed so that material is uniformly delivered at the desired moisture content. Rotary dryers work well for material that has limited fluidity. Continuous gravity flow dryers, or cross flow dryers, reduce moisture by blowing air across a column of material. Cross flow dryers work well for materials that flow easily and permit airflow between individual components (i.e. pellets).Batch or bin driers force air through a static material in a bin. Material to be bin dried must sufficiently resistant to compression to allow proper void space for air flow. Finally, biomass like algae may require unique dryers to remove water from a suspension (e.g. Like in a spray dryer) which uses the difference in air and solution moisture equilibrium.

In all drying, the rate of water removal depends on the conditions of the air, the properties of the biomass, and the design of the dryer. Moisture in the biomass can be held in varying degrees of bonding; easily-removed water is referred to as free water and more tightly-retained water referred to as bound water.

\subsubsection{Densification}

Densification process converts the feedstock into a pellet to achieve consistent physical properties such as size and shape, bulk and unit density, and durability which significantly influence storage, transportation and handling characteristics, resulting in reduced feedstock cost and increased quality. A variety of densification systems are considered for producing a uniform format feedstock commodity for bioenergy applications, such as pellet mill, cuber, screw extruder, briquette press, roller press, tablet press, and agglomerator. Each of these systems has varying impacts on feedstock chemical composition physical properties, and energy consumption.

\subsubsection{Conventional Pelletizing}

Conventional biomass pellet production (Figure 21) includes initial size reduction to a 2- in. particle size, followed by drying to 10 to $12 \%$ moisture content (wet basis) using a rotary dryer. The dried biomass is then passed through a second stage grinding process to reduce the particle size to less than 3/16-in. (typically to $2 \mathrm{~mm}$ ), steam conditioned, and pelletized ${ }^{42}$. Drying is the 
major energy consumption unit operation in this process, accounting for about $70 \%$ of the total pelletization energy.

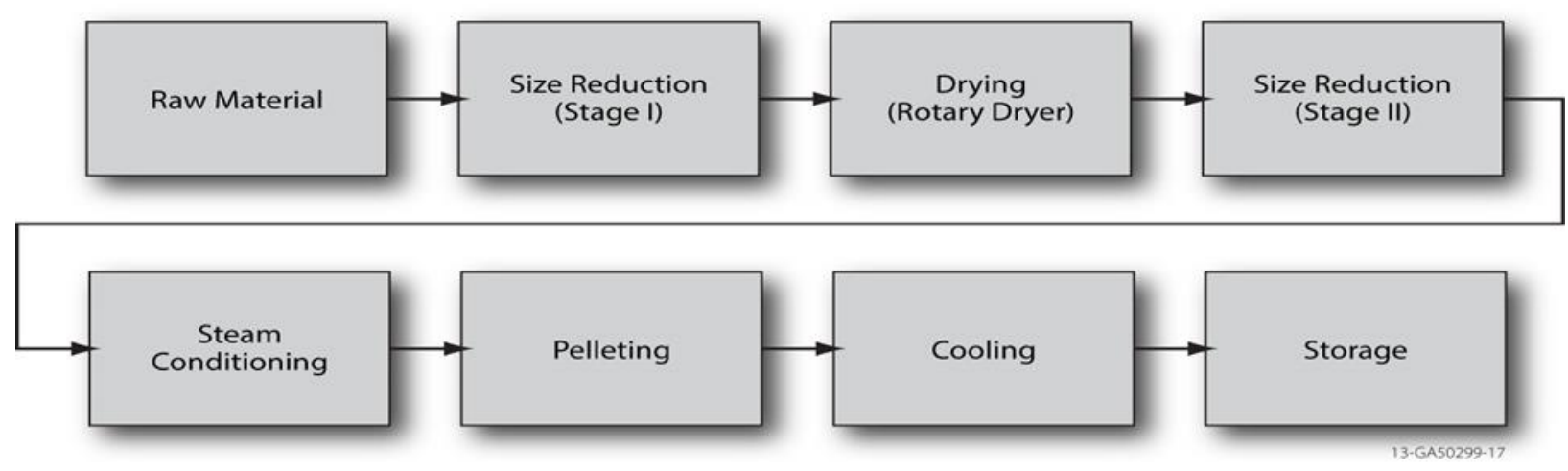

Figure 21. Conventional pelletization process.

\subsubsection{High-Moisture Densification Design Basis}

Significant cost reductions to the conventional drying and pelleting processes are possible with a process of high-moisture densification (that is under development at INL) that eliminates the energy intensive rotary drying process prior to pelleting. In this process, the high-temperature (typically 160 to $180^{\circ} \mathrm{C}$ ) drying step is replaced with a low-temperature (approximately $110^{\circ} \mathrm{C}$ ), short duration (typically several minutes) preheating step. The combination of preheating with the additional frictional heat generated in the pellet die results in a reduction of feedstock moisture content by about 5 to 10 points (e.g., from $30 \%$ down to 25 to $20 \%$ ). The pellets produced still have high moisture and require further drying to about $7 \%$ for safe storage and transportation (Figure 22). It should also be noted that higher moisture densification does not include the addition of a binder.

This process has been demonstrated at INL where corn stover, ranging in moisture from 28 to $38 \%$, was preheated at $110^{\circ} \mathrm{C}$ for 3 to 4 minutes prior to pelleting in a laboratory flat-die pellet mill using both 8 and $6 \mathrm{~mm}$ dies. The pellets exited the mill at 20 to $30 \%$ moisture content and, after drying, exhibited densities greater than $30 \mathrm{lb} / \mathrm{ft}^{3}$ and disabilities greater than $95 \%$. The specific energy consumption was found to be in the range of 40 to $100 \mathrm{kWhr} / \mathrm{ton}^{43}$.

The reduction in drying energy is the key advantage of this approach. First, the process uses the heat generated in the pellet die to partially dry the material. Second, drying the pellets offers cost and energy advantages over drying loose, bulk material. Loose biomass typically is dried in a concurrent flow rotary dryer. Rotary biomass dryers typically operate at temperatures of about 150 to $160^{\circ} \mathrm{C}$, have greater particulate emissions, greater volatile organic compound emissions, greater fire hazard, a large footprint, and often have difficulty in controlling the material moisture. With the increased density, the reduced tendency for material to become entrained in the air flow, and the increased heat transfer coefficients compared to loose biomass, more efficient drying technologies options are available for drying pellets. A cross-flow dryer (common in grain drying) operates at temperatures less than $100^{\circ} \mathrm{C}$, reduces the particulate and 
volatile organic compound emissions, and will have better temperature distribution. A comparison of pellet properties and energy balances for conventional and high-moisture pelletization processes is given in Error! Reference source not found.. The table shows 2017 Design Case targets to achieve a 40 to $50 \%$ reduction in the total pelletization and drying energy.

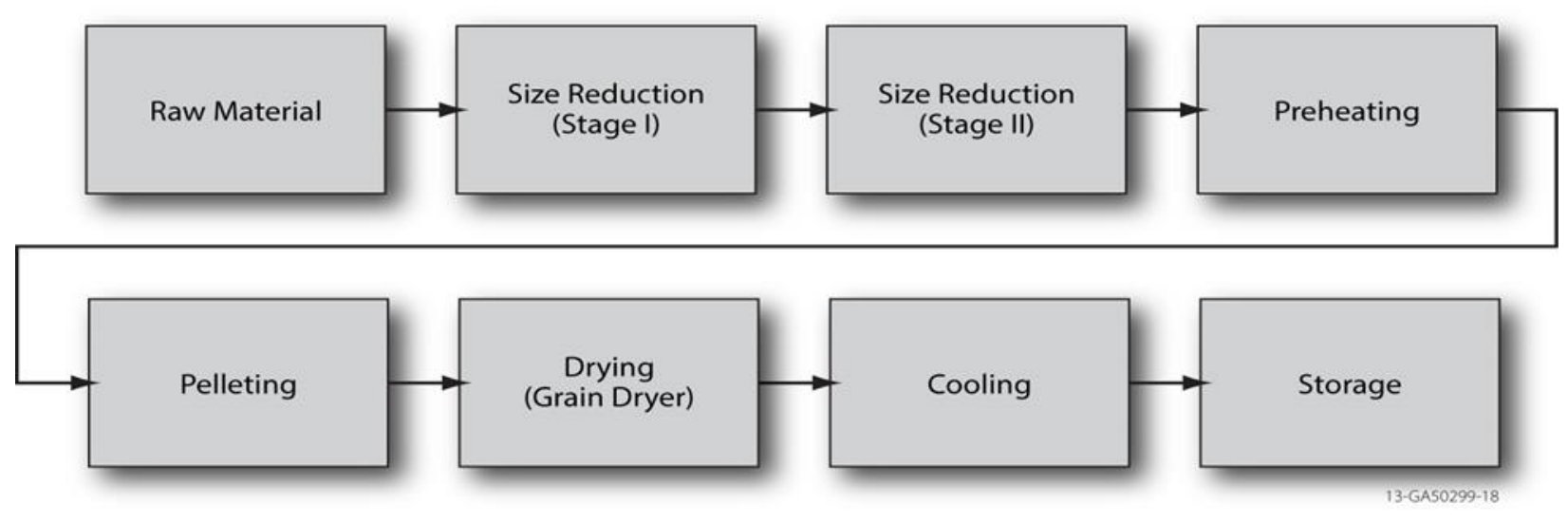

Figure 22. High-moisture pelletization process.

Table 7 Drying and densification design basis

\begin{tabular}{|lll|}
\hline Moisture Properties & 2013 SOT & 2017 Target \\
\hline Infeed Moisture & $30 \%$ & $30 \%$ \\
\hline Dryer Moisture Reduction & $18 \%$ & $11 \%$ \\
\hline Densification Moisture Reduction & $3 \%$ & $10 \%$ \\
\hline Final Pellet Moisture & $9 \%$ & $9 \%$ \\
\hline Densification Energy & $75 \mathrm{kWhr} / \mathrm{dry} \mathrm{T}$ & $50 \mathrm{kWhr} / \mathrm{dry} \mathrm{T}$ \\
\hline Drying Energy & $350 \mathrm{kWhr} / \mathrm{ton}$ & $100 \mathrm{kWhr} / \mathrm{ton}$ \\
\hline Pellet Properties & & \\
\hline Unit Density & $70 \mathrm{lb} / \mathrm{ft}^{3}$ & $65 \mathrm{lb} / \mathrm{ft}^{3}$ \\
\hline $\begin{array}{l}\text { Bulk Density } \\
\text { Durability }\end{array}$ & $40 \mathrm{lb} / \mathrm{ft}^{3}$ & $35 \mathrm{lb} / \mathrm{ft}^{3}$ \\
\hline
\end{tabular}

The high-moisture densification design basis assumptions are as follows:

Our preliminary studies indicated that it is possible to produce high-quality pellets using corn stover; however, for our 2017 Design Case, we are assuming that the process works for other woody and herbaceous feedstocks to produce durable, high-density pellets.

- Technical and cost targets are estimated with the assumption that a grain dryer will be used to dry high-moisture pellets. 
- Drying of pellets using energy-efficient driers like grain and belt driers is more economical compared to conventional rotary driers.

- Slow drying at low temperatures of less than $60^{\circ} \mathrm{C}$ can result in more uniform moisture distribution in pellets.

\subsection{Transportation}

Transportation includes all processes involved in the movement of material from multiple locations to a centralized location (such as a preprocessing facility or depot or biorefinery). Processes include loading, trucking, rail transport, and unloading. Whereas transportation relies on existing roadways, railways, and waterways to move biomass, collection requires the use of specialized machinery to navigate off-road, gather dispersed biomass from a field or stand, and move it to the nearby staging location. In terms of biomass, the goal of transportation is to strategically utilize existing systems efficiency and cost effectively. A national scale biorefinery industry will require movement of great amounts of biomass and therefore understanding capacity and time constraints are important issues to consider. Long distance transportation like rail and barge allow biomass to be transported greater distances but this incurs additional costs. Densification of material both in particle size and energy content (i.e. torrefied material) allow for some of these costs to be offset. Unit trains or dedicated transportation of a set amount of material are additional strategies to be considered to reduce cost but the use of these options are constrained by location, timing and capacity. Differing transportation modes such as rail and barge can be cost efficient for long distance and pellet transportation. Understanding rail movement of freight rail is inherently more complex than estimating costs for trucking, as rail has to consider many factors such as distance, rail car type, car ownership, switching activities, shipment volume type, etc.

\subsection{Handling and Queuing}

Handling and queuing involves processes required to move biomass material to inside the gates at the biorefinery to the throat of the conversion. Handling equipment can include circular pile reclaimers, loaders, surge bins, conveyors, dust collection, pellet crumblers, and miscellaneous equipment required to move, queue and consistently deliver material. Handling operations are influenced by type of feedstock and depend on many factors, including moisture content, bulk density, particle size and shape distribution. Feedstocks, like corn stover, inherently possess characteristics that inhibit handling (such as high cohesivity, low density, high compressibility, and high variability in particle size and shape uniformity) ${ }^{8}$. For this reason, feedstock handling operations are typically designed at $150 \%$ of expected capacity. Handling issues can be improved by either engineering new equipment or engineering the feedstock (uniform particles or densified materials). Strategies to mix or blend multiple feedstock for cost and quality mitigation will require additional challenges handling multiple feedstock. For example formulated feedstocks will require more complex feed operations, similar to the food industry, that are capable of consistently delivering a homogeneous and precise ratio of desired material. 


\section{Supply System Design: Arrangement of Unit Operations}

Organizing feedstock logistics in a way that maintains economic and environmental sustainability, while providing necessary resource quantities, is a principal challenge that needs to be addressed before a self-sustaining industry can evolve. Strategies can be taken in three ways to organize the earlier mentioned supply operations to reduce transportation distance, to reduce supply risk, and to increase the biomass resource. Supply chain unit operations of feedstock logistics can be configured depending on existing biomass and future commodity scale biomass markets. In this section we present conventional and advance supply system.

\subsection{Conventional Feedstock Supply System}

The conventional feedstock supply system design is based on practices currently developed for both the conventional forestry and agriculture industries, and is economical in high-yielding areas (for example, a corn stover production system in Iowa). The defining characteristic of the conventional feedstock system is the arrangement of the unit operations which puts preprocessing behind the biorefinery receiving gate where each biorefinery is designed to accept a specific local feedstock (Figure 23).In this organization of operations, the burden of adapting to feedstock resources is assumed primarily by the biorefinery.

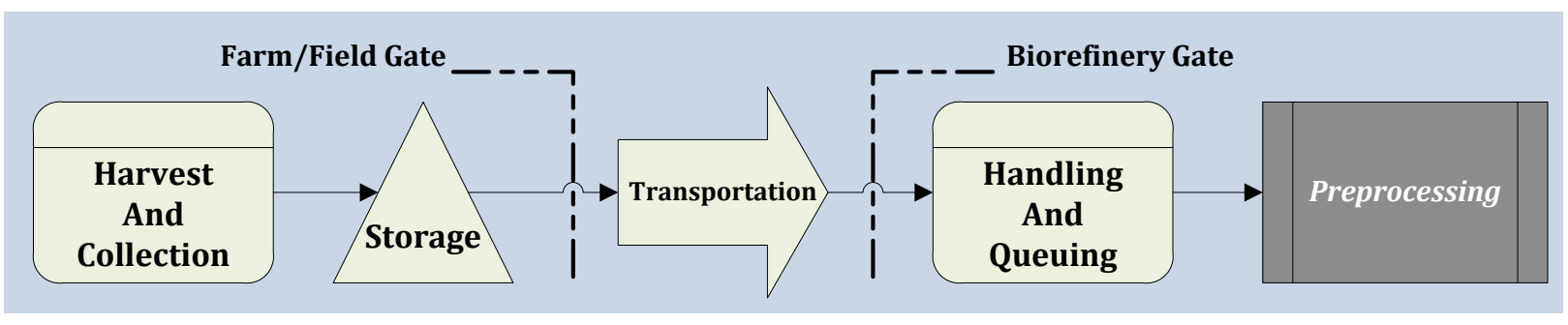

Figure 23. Conventional feedstock system for herbaceous lignocellulosic biomass.

In a conventional design, materials are stored on the field without further processing before transportation and handling operations can move material from long-term storage to shorterterm, storage at the biorefinery. Preprocessing operations are located at the biorefinery which include all milling, conveyance, dust collection, and biomass material surge systems is necessary to reduce biomass size before insertion in the conversion process (e.g., biochemical or thermochemical). With this arrangement of unit operations, biorefineries will be designed to accept a specific local feedstock or feedstocks and will need to undertake the burden of modifying the resource to a usable form.

\subsection{Advanced Supply System}

The advanced supply system concept arranges preprocessing technologies to mitigate the density and stability issues that prevent biomass from being handled in high-efficiency bulk dry solid or liquid distribution systems. The advanced supply system produces a feedstock supply system in which the diversity of biomass formats is minimized as early in the supply system as practically possible through a series of preprocessing steps. The organization of the unit operations in an advanced supply system allows preprocessing to occur during harvest and collection and/or at centralized preprocessing sites (depots), which are envisioned to resemble existing systems like 
the grain elevator or beet dump. Advanced supply system relies on densifying biomass at local preprocessing facilities before delivering to a biorefinery and before long distance transportation. From the depot, the downstream feedstock supply systems and infrastructure will become uniform commodity-scale equipment and handling systems (Figure 24).

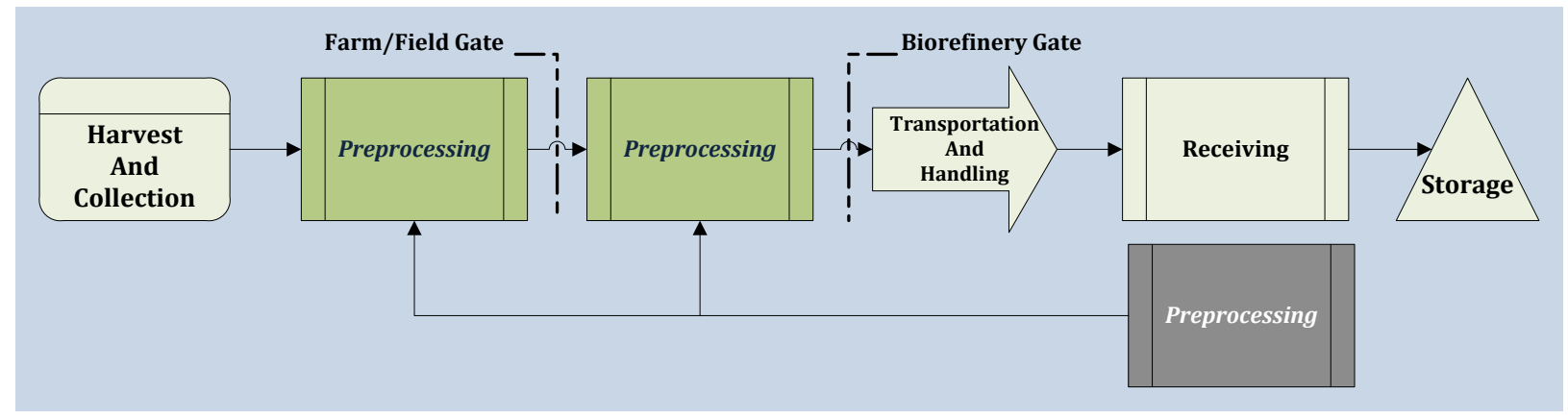

Figure 24. Advanced supply system designs (Advanced Uniform) follow the model of the current grain commodity supply system, which manages crop diversity at the point of harvest and/or depot, allowing all subsequent feedstock supply system infrastructure to be similar for all biomass resources ${ }^{4}$.

The purpose of the drying and densification at the advanced supply system is to achieve consistent properties, such as: size and shape, bulk and unit density, and durability. These properties significantly influence storage, transportation, and handling characteristics and, by extension, feedstock cost and quality. The drying and densification conducted under an advanced supply system converts the raw biomass into a biomass pellet.

In the advanced supply system, it is expected that biomass will be trucked from a local draw radius to a depot where preprocessing addresses quality (moisture content, ash content) and density issues to allow more efficient high capacity transportation and handling. The advanced supply system assumes that there will be insignificant material loss once preprocessing is completed, and transportation will not be intensively constrained by volume (as a result of low bulk densities). Additionally, densification increases material uniformity and flow ability, which is advantageous in transportation activities allowing for long distance transportation mode such as rail and barge. The fundamental premise of the advanced supply system design is that highcapacity, high-efficiency supply systems already exist (e.g., grain and petroleum crude) and handling low-density/aerobically unstable material is inherently inefficient, which can be mitigated through innovative organization of the unit operations within the supply system. 


\section{Biological Conversion of Sugars to Hydrocarbons}

The following sections outline the tailored logistic designs including costs and performance specifications designed to meet the $\$ 80$ per dry ton costs for Biological Conversion of Sugars to Hydrocarbons pathway. This conversion path is coupled with the National Renewable Energy Laboratory's (NREL's) hydrocarbon design report, "Dilute-Acid Prehydrolysis and Enzymatic Hydrolysis Deconstruction of Biomass to Sugars and Biological Conversion of Sugars to Hydrocarbons," ${ }^{44}$ that describes a viable route from biomass to hydrocarbon fuels. Because of this coupling, the assumptions of scale and feedstock quality requirements are consistent with the design case assumptions used by NREL in their report and techno economic assessments. In addition, this design does not consider the different requirements and nuances of other biological conversion processes or other hydrocarbon pathways.

\subsection{Summary}

Two requirements for the 2017 Design Case that were established early in this report are: achieving the $\$ 80$ per dry ton cost target when located outside the Midwest Corn Belt and achieving biorefinery quality specifications within the $\$ 80$ per dry ton cost target. Feedstock curves were developed for the 2017 Design Case scenario located in western Kansas (Figure 25). These curves included access costs (i.e., grower payment), logistics costs, and dockage costs (e.g., ash and carbohydrate dockage). Using these curves, it was determined that a feedstock blend of $60 \%$ corn stover, 35\% switchgrass, and 5\% MSW would meet the $\$ 80$ per dry ton delivered feedstock cost target, thus satisfying the cost criterion of the 2017 Design Case (see Error! Reference source not found.). 
Table 8 Biochemical conversion feedstock design cost analysis

\begin{tabular}{|c|c|c|c|c|c|}
\hline Cost Element & $\begin{array}{l}\text { Single-pass } \\
\text { Corn Stover }\end{array}$ & $\begin{array}{l}\text { Multi-pass } \\
\text { Corn Stover }\end{array}$ & Switchgrass & MSW & Blend \\
\hline Formulation Contribution & $35 \%$ & $25 \%$ & $35 \%$ & $5 \%$ & - \\
\hline Grower payment/Access Cost & 27.20 & 27.20 & 29.80 & 18.00 & 27.70 \\
\hline Harvest and collection ( $\$ /$ dry $T)$ & 10.50 & 19.20 & 15.40 & - & 13.90 \\
\hline Transportation (\$/dry T) & 8.70 & 8.30 & 7.20 & 18.00 & 8.60 \\
\hline Preprocessing (\$/dry T) & 23.40 & 23.40 & 19.70 & 19.70 & 21.90 \\
\hline Storage $(\$ /$ dry $T)$ & 6.50 & 6.50 & 5.50 & 4.50 & 6.10 \\
\hline Handling (\$/dry T) & 1.80 & 1.80 & 1.80 & 1.80 & 1.80 \\
\hline $\begin{array}{l}\text { Total Delivered Feedstock Cost } \\
(\$ / \text { dry } T)\end{array}$ & 78.10 & 86.40 & 79.40 & 62.00 & $\sim 80.00$ \\
\hline \multicolumn{6}{|l|}{ Delivered Feedstock Specifications* } \\
\hline Ash content (wt. \%) & 3.5 & 7 & 4 & 10 & 4.9 \\
\hline Moisture content (\%, wet basis) & 9 & 9 & 9 & 9 & 9 \\
\hline Carbohydrate content (wt. \%) & 64 & 57 & 57 & 57 & 59 \\
\hline
\end{tabular}




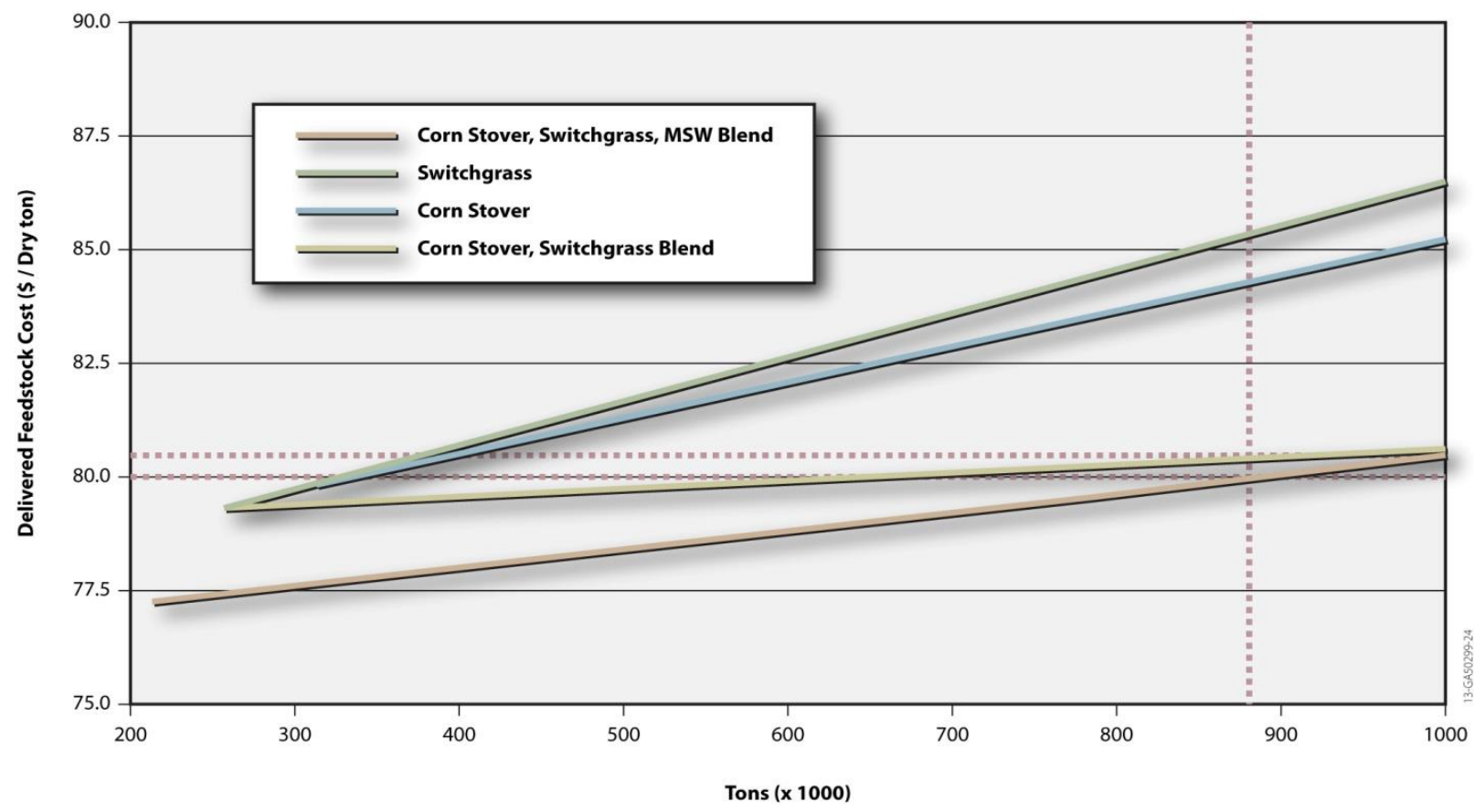

Figure 25. Comparison of individual and blended feedstock costs. A blend of $60 \%$ corn stover, $35 \%$ switchgrass, and 5\% municipal solid waste is needed to hit the $\$ 80$ feedstock cost target.

Even though feedstock quality is represented in the cost curves with a dockage fee (in this case, ash dockage for multi-pass corn stover and MSW ash content in excess of the 5\% ash specification), the least-cost formulation approach does not guarantee that the lowest-cost feedstock meets spec. In fact, the $60 \%$ corn stover, $35 \%$ switchgrass, and 5\% MSW blend actually exceeded the ash specification with blended ash content of $6.1 \%$. As a result it was necessary to replace some of the higher-ash, multi-pass stover with lower-ash, single-pass corn stover in order to meet the ash specification (Table 9). The rationale for including both single and multi-pass stover is that because single pass technology is a new technology requiring additional investment by farmers, it is unlikely it will fully replace multi-pass harvest by 2017 . Sourcing $35 \%$ single-pass and $25 \%$ multi pass corn stover assumes that about $60 \%$ of the stover will be single-pass and $40 \%$ will be multi-pass. This seems to be a reasonable assumption considering that the $60 \%$ may be harvested by a custom harvester and $40 \%$ by local farmers.

For the 2017 Design Case scenario located in western Kansas, both the cost and quality criteria could be achieved through blending. However, there may be other scenarios where reaching the $5 \%$ ash specification for biochemical conversion will require the removal of silica. Methods for accomplishing silica removal include both fine grinding followed by triboelectrostatic separation and alkali-based processes that dissolve silica ${ }^{45}$. A recent analysis for non woody feedstocks estimated a net cost of $\$ 39.93$ to $\$ 60.80$ /dry T for removal of alkali metals (up to $95 \%$ ) by leaching, followed by removal of silica (up to $75 \%$ ) by triboelectrostatic separation ${ }^{45}$. With an $\$ 80 /$ dry T feedstock cost target, these costs are too high to allow the use of chemical preconversion as an added unit operation in the current design; the existing feedstock supply 
chain operations and the grower payment leave little room for added cost. A detailed discussion of chemical preconversion for ash removal is included in Appendix D. Therefore, for this report, we have selected feedstocks that can meet the ash specification in a blend with MSW.

The moisture and carbohydrate content of the blended feedstock also meet the specification for moisture content (i.e., less than 20\%) and carbohydrate content (i.e., at least 59\%). Because each feedstock is pelletized prior to blending, the pellets are dried to about 9 to $10 \%$ during pellet production, thereby fixing the moisture content of the blend. Similar to ash content, the carbohydrate specification is met by blending. The carbohydrate content of MSW varies depending on the particular fraction, ranging from $46 \%$ for yard waste to $64 \%$ for food waste. The MSW carbohydrate content shown in Table 8 is the average of yard waste $(46 \%)$, food waste $(64 \%)$, non-recyclable paper (55\%), and C\&D waste (61\%). Because MSW is such a small fraction of the overall blend, even food waste blends out to a carbohydrate content of $59 \%$.

\subsection{Feedstock Composition (In-feed quality specifications)}

The conversion target for feedstock composition remains consistent with what was assumed in the 2011 and 2013 design cases; however, the biomass supply composition is updated relative to the original 2011 case. Previous requirements for feedstocks assumed in the 2011-era ethanol models included cost targets only for a single biorefinery. There were no quality requirements; further analysis indicated a mismatch on biomass characteristics and conversion in-feed specifications. Furthermore, this design case focused more on supplying industry level biomass supply versus a single biorefinery. The update applied in the 2013 design case and maintained here improves on the 2011 design basis and the associated original feedstock price of $\$ 58.50 /$ dry ton $(2007 \$)$, which included a more uncertain grower payment, to a more reasonable target at an increased price of $\$ 80 /$ dry ton including grower payment (2011\$). This new price is more appropriate for a large commodity scale going beyond a "niche market" price.

As has been described in prior conversion design reports, the feedstock composition (Table 9) plays a critical role on overall process design and economics, primarily with respect to carbohydrate components (cellulose and hemicellulose), lignin, and increasingly acetate and ash, given modifications being made to the pretreatment strategy such as the use of deacetylation, as well as high sensitivity to impurity components such as ash and metals in the catalytic reactor section of this design. The blended uniform-format feedstock composition assumed here for purposes of future design case targets is shown below, with supporting details (in the context of corn stover compositional variability) described in the 2011 ethanol report ${ }^{46}$. Also consistent with prior design cases, the moisture content for the delivered feedstock is $20 \%$ or less. 
Table 9 Delivered Feedstock Composition Assumed in the Present Design ${ }^{44}$.

\begin{tabular}{|lc|}
\hline Component & $\begin{array}{c}\text { Composition } \\
\text { (dry wt \%) }\end{array}$ \\
\hline Glucan & 35.1 \\
\hline Xylan & 19.5 \\
\hline Lignin & 15.8 \\
\hline Ash & 4.9 \\
\hline Acetate & 1.8 \\
\hline Protein & 3.1 \\
Extractives & 14.7 \\
\hline Arabinan & 2.4 \\
Galactan & 1.4 \\
\hline Mannan & 0.6 \\
Sucrose & 0.8 \\
Total structural carbohydrate & 59.0 \\
Total structural carbohydrate + sucrose & 59.8 \\
\hline Moisture (bulk wt \%) & 20.0 \\
\hline
\end{tabular}

${ }^{a}$ Represents acetyl groups present in the hemicellulose polymer; converted to acetic acid in pretreatment.

The current design is assuming an ash target of $4.9 \%$, structural carbohydrate of $59 \%$ and a moisture of $<20 \%$.

\subsection{Feedstock Selection Cost Estimation}

Access costs are calculated from the farm gate cost based on county level supply costs as shown in Figure 26, which are derived from The Billion Ton Update ${ }^{5}$ data and are available from the Bioenergy Knowledge Discovery Framework ${ }^{25}$. Farm gate costs include nutrient replacement costs, harvest and collection costs, and an additional "profit" equal to $15 \%$ of nutrient replacement costs ${ }^{47}$. Access cost (grower payment) is calculated by subtracting the biomass harvest and collection costs from the farm gate costs. While the results are produced from ORNL's analysis using POLYSYS, the harvest and collection costs are supplied by the INL's Biomass Logistics Model. 


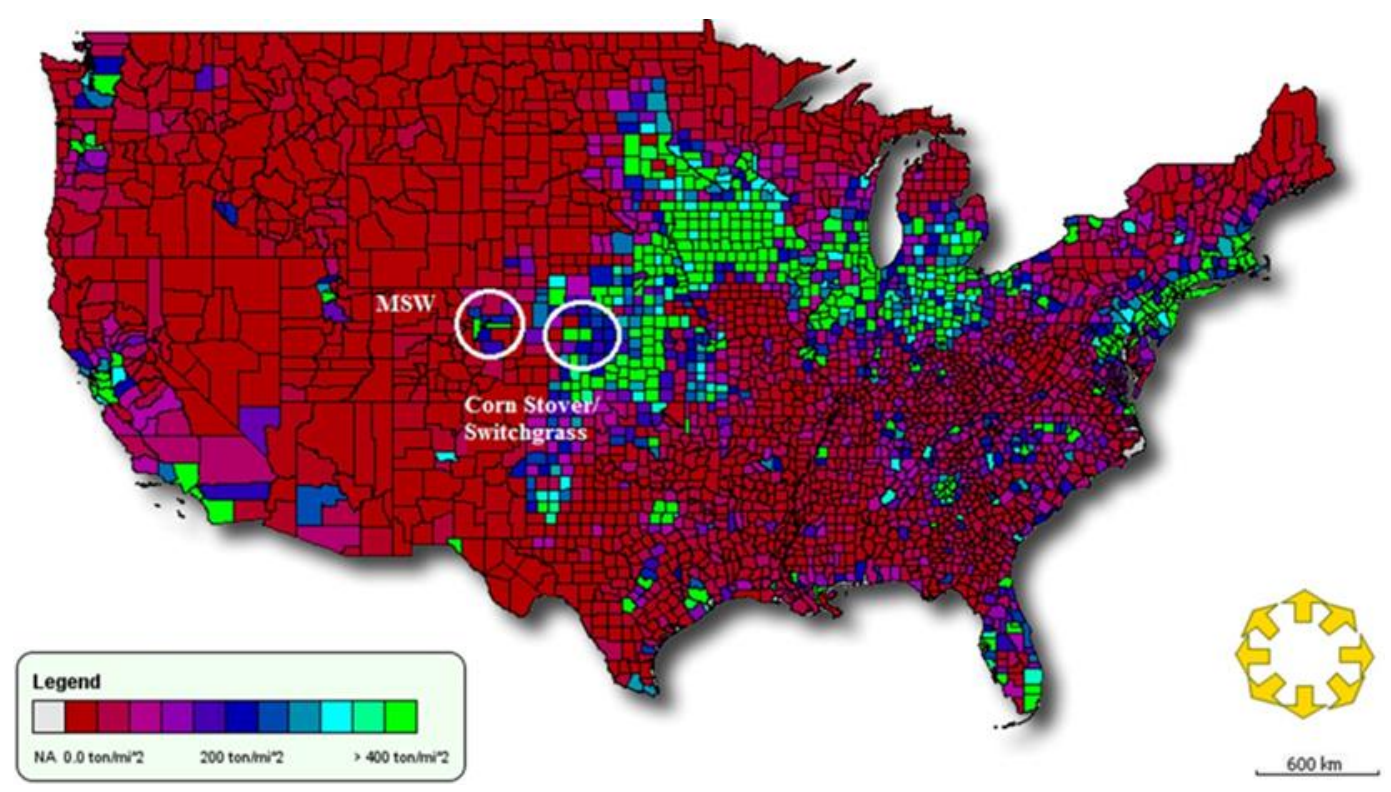

Figure 26. Resource selections for the 2017 Design Case to support biochemical conversion. Figure shows tonnages available at $\$ 40 /$ dry ton. Green represents higher amounts of tonnage available, red represents no available tonnage available at $\$ 40 /$ dry ton ${ }^{47}$.

The 2017 Design Case basis discussion presented above provided the least-cost formulation approach for reducing access costs by accessing multiple feedstocks. With this approach, reduced quantities of each feedstock that make up the total blendstock allows us to stay lower on the supply curve than if we had to supply the entire supply demand with any single feedstock. The impact of this approach is shown in Table 10. The 2013 SOT assumes a 100\% supply of corn stover and an access cost to supply 870,000 dry $\mathrm{T}$ estimated at $\$ 40 /$ ton. In comparison, the 2017 Design Case blend of 60\% corn stover, 35\% switchgrass, and 5\% MSW results in a weighted average feedstock cost of $\$ 27 /$ dry ton that is nearly $30 \%$ lower than the access cost of stover alone.

Table 10. Resource farm gate price

\begin{tabular}{|c|c|c|c|c|}
\hline & \multicolumn{2}{|l|}{2013 SOT } & \multicolumn{2}{|l|}{2017 Target } \\
\hline & $\begin{array}{l}\text { Access Cost (2011 } \\
\text { \$/dry T) }\end{array}$ & Tons & $\begin{array}{l}\text { Access Cost (2011 } \\
\text { \$/dry T) }\end{array}$ & Tons \\
\hline $\begin{array}{l}\text { Corn stover } \\
\text { farmgate price }\end{array}$ & 40.00 & 870,000 & 27.20 & 522,000 \\
\hline Switchgrass & NA & 0 & 29.80 & 304,500 \\
\hline $\begin{array}{l}\text { MSW cost to } \\
\text { purchase and } \\
\text { segregate }\end{array}$ & NA & 0 & 18.00 & 43,500 \\
\hline Total & 40.00 & 870,000 & 27.65 (wt avg) & 870,000 \\
\hline
\end{tabular}




\subsection{Quality Specification and Design Assumptions}

The major assumptions of the 2017 Design Case, compared to the 2012 Conventional Design and the 2013 SOT are shown in Table 11. The implications of these assumptions on feedstock supply systems designs are discussed in following sections of the report.

Table 11. Summary of assumptions underpinning progressive design implementations

\begin{tabular}{|c|c|c|c|}
\hline & 2012 Conventional Design & 2013 SOT & 2017 Design Case \\
\hline Feedstock(s) & Corn stover & Corn stover & $\begin{array}{l}\text { Blended feedstock: corn } \\
\text { stover, switchgrass, and select } \\
\text { municipal solid waste (MSW) }\end{array}$ \\
\hline Grower payment & Minimal & $\begin{array}{l}\text { Increases based on marginal } \\
\text { cost differential }\end{array}$ & $\begin{array}{l}\text { Calculated and modeled } \\
\text { according to specific location } \\
\text { and resource } \\
\text { blend/formulation }\end{array}$ \\
\hline Moisture & Field dried to $12 \%$ & $\begin{array}{l}\text { Arrives at } 30 \% \\
\text { Dried to } 20 \%\end{array}$ & $\begin{array}{l}\text { Arrives: corn stover } 30 \% \text {, } \\
\text { switchgrass } 20 \% \text {, and } \\
\text { MSW } 20 \% \text {; } \\
\text { All dried to } 7 \%\end{array}$ \\
\hline Ash & No ash management assumed & $\begin{array}{l}11 \% \text {, dockage accessed for ash } \\
\text { content Greater than } 5 \% \text { spec }\end{array}$ & $\begin{array}{l}\text { Corn stover: multi-pass } 7 \% \text {; } \\
\text { single-pass } 3.5 \% \\
\text { Switchgrass: } 4 \% \\
\text { MSW: } 10 \% \\
\text { Blended ash content of } 4.9 \%\end{array}$ \\
\hline Logistics & Uses existing systems & Uses existing systems & $\begin{array}{l}\text { Fractional milling } \\
\text { High-moisture densification } \\
\text { Rail transportation for MSW }\end{array}$ \\
\hline $\begin{array}{l}\text { Quality controls } \\
\text { (passive) }\end{array}$ & $\begin{array}{l}\text { Field drying to meet moisture } \\
\text { spec } \\
\text { Ample available resource; } \\
\text { quality spec manually selected }\end{array}$ & $\begin{array}{l}\text { Dockage fee assessed to } \\
\text { supplier for below-quality } \\
\text { material }\end{array}$ & $\begin{array}{l}\text { Multi versus single-pass } \\
\text { harvest/ collection } \\
\text { Harvest/collection and storage } \\
\text { best management practices }\end{array}$ \\
\hline $\begin{array}{l}\text { Quality controls } \\
\text { (active) }\end{array}$ & None assumed & Rotary drying & $\begin{array}{l}\text { Multiple resource } \\
\text { blending/formulation } \\
\text { High-moisture densification } \\
\text { High-efficiency pellet drying }\end{array}$ \\
\hline Meets quality target & No & Yes & Yes \\
\hline Meets cost target & Yes & No & Yes \\
\hline $\begin{array}{l}\text { Accesses dispersed } \\
\text { resources }\end{array}$ & No & No & Yes \\
\hline
\end{tabular}

\subsection{Feedstock Logistics}

\subsubsection{Harvest and Collection}

\subsubsection{Overview}

The 2012 Conventional Design focused on conventional multi-pass harvest methods (i.e., the mowing and/or windrowing operations are separate from the baling operation). Single-pass harvesting systems (such as those developed through the DOE-funded, high-tonnage, logistics projects) offer efficiency and quality improvements over conventional, multi-pass systems. The 2017 Design Case assumes that the immaturity of the biomass market will limit the farmer investment in advanced equipment options. Therefore, with the exception of a few proactive, 
early adopters, conventional, multi-pass systems will dominate the marketplace in the regions defined by the 2017 Design Case.

In the 2017 Design Case, corn stover is harvested using a flail shredder, which is commonly referred to as a stalk chopper. The ability of a stalk chopper to minimize soil pickup and contamination compared to alternate methods drives this decision ${ }^{48}$. Corn stover harvest occurs within a 6-week window that coincides with grain harvest. In this operation, stalk chopping and baling (i.e., $3 \times 4 \times 8$-ft large, square bales) immediately follow grain harvest. The 2017 Design Case assumes a stalk chopper collection efficiency (i.e., removal rate) of about $40 \%$, with a corn stover moisture content up to $30 \%$ (wet basis). It also assumes that field drying to a preferred moisture content (i.e., less than $20 \%$ ) for long-term storage may not always be possible, resulting in corn stover bales with up to $30 \%$ moisture content that must be appropriately managed during storage. While drying in storage may occur, high-moisture biomass undergoes dry matter loss early in storage, resulting in both feedstock loss and compositional changes ${ }^{22}$.

Switchgrass harvest in the 2017 Design Case also follows conventional practices. Following plant senescence in the fall, when plant nutrients retreat into the root system and the plant naturally dries down, switchgrass is cut and windrowed using a self-propelled mowerconditioner; then it is subsequently baled using a large-square (i.e., $3 \times 4 \times 8$ - $\mathrm{ft}$ ) baler. A collection efficiency of $90 \%$ and bale moisture content of less than $20 \%$ is assumed in cost estimation for switchgrass harvest and collection.

\subsubsection{Harvest and Collection Design Basis}

The 2017 Design Case uses traditional harvest and collection equipment (i.e., both multi-pass and single-pass methods); however, it depends heavily on advancing operational strategy. Maintaining the design specifications shown in Table 12 for ash and moisture content requires a balance between harvested biomass quality, storage behavior, and final delivered feedstock specification. The blending strategy used by the 2017 Design Case merges the benefits of multipass and single-pass systems to enforce ash avoidance during harvest and reduce ash enrichment throughout storage. To meet the delivered feedstock specifications, the harvest and collection task relies on the adjustment of pre-storage goals for ash content, moisture content, and biomass yield.

Table 12.Technical targets for harvest and collection of herbaceous resources in the 2017 Design Case

\begin{tabular}{lllllll}
\hline & Ash Content & \multicolumn{2}{c}{ Baled Moisture } & \multicolumn{2}{c}{ Bulk Density } \\
Process & 2013 SOT & 2017 Target & 2013 SOT & 2017 Target & 2013 SOT & 2017 Target \\
\hline Multi-pass & $10 \%$ & $7 \%$ & $30 \%$ & $30 \%$ & $12 \mathrm{lb} / \mathrm{ft}^{3}$ & $12 \mathrm{lb} / \mathrm{ft}^{3}$ \\
Single-pass & $6 \%$ & $3.5 \%$ & $30 \%$ & $30 \%$ & $12 \mathrm{lb} / \mathrm{ft}^{3}$ & $12 \mathrm{lb} / \mathrm{ft}^{3}$ \\
Switchgrass & $6 \%$ & $4 \%$ & $20 \%$ & $20 \%$ & $12 \mathrm{lb} / \mathrm{ft}^{3}$ & $12 \mathrm{lb} / \mathrm{ft}^{3}$ \\
\hline
\end{tabular}


The 2017 Design Case focuses on improvements to and optimization of conventional equipment. Single-pass and advanced, multi-pass harvesting systems (i.e., specialized combine operation or windrowing equipment) that provide the lowest ash content feedstock will emerge first in the highly productive regions, where the economics of a single-feedstock market allow farmers to spread their investment across more acres and tons of biomass. In less productive areas, conventional multi-use systems will be operated with greater focus on reducing feedstock moisture content and improving storage stability to avoid ash enrichment throughout storage. Idaho National Laboratory (INL) research shows that stover ash content from conventional multi-pass collection equipment can approach the 2017 goal of $7.5 \%$ ash. However, additional improvements are required to minimize the uncertainty of soil entrainment while maximizing biomass yield and sustainability.

\subsubsection{Harvest and Collection Cost Estimation}

Harvest and collection costs assume a sustainable removal rate of 1.2 dry T/acre for corn stover (both single and multi-pass) and 5-dry T/acre for switchgrass. These assumptions are consistent with those used in the Billion Ton Update ${ }^{5}$. Cost reductions from the 2013 SOT to the 2017 Design Case are largely attributed to the transition from multi-pass corn stover harvest in the 2013 SOT to single-pass harvest in the 2017 Design Case (Table 13). These cost reductions are attributed to both a reduction in ash, and an improvement in the overall efficiency of the harvest operations that result from single-pass harvesting. The cost of ash is estimated from the ash dockage $\$ 2.25 /$ dry T per percent ash presented in Section 2.2.2. Ash dockage contributed $\$ 14 /$ dry T to the 2013 SOT costs. Cost of each machine consists of ownership cost and operations cost. Details are listed in Appendix E. However, the 2017 Design Case assumes that, with improvements to multi-pass harvest systems and through increased adoption of single pass harvesting equipment, the blended feedstock ash content is within specifications, thereby eliminating an ash dockage. Additional cost savings are realized through improved bale densities that result from anticipated improvements in the high-density baling technology. 
Table 13. Biomass harvest and collection cost estimates.

\begin{tabular}{|c|c|c|}
\hline Machine & $\begin{array}{c}2013 \text { SOT } \\
(2011 \text { \$/dry T) }\end{array}$ & $\begin{array}{c}2017 \text { Target (2011 } \\
\text { \$/dry T) }\end{array}$ \\
\hline & Total & Total \\
\hline \multicolumn{3}{|l|}{ Multi-pass corn stover } \\
\hline Combine* & 0.00 & 0.00 \\
\hline Shredder & 5.30 & 5.30 \\
\hline Baler & 10.60 & 10.60 \\
\hline Bale collection/stacking & 3.30 & 3.30 \\
\hline Ash dockage & 14.00 & 0.00 \\
\hline Totals & 33.20 & 19.20 \\
\hline \multicolumn{3}{|l|}{ Single-pass corn stover } \\
\hline Combine* & 0.00 & \\
\hline Baler & 7.20 & 7.20 \\
\hline Bale collection/stacking & 3.30 & 3.30 \\
\hline Totals & 10.50 & 10.50 \\
\hline \multicolumn{3}{|l|}{ Switchgrass } \\
\hline Mower-conditioner & 4.80 & 4.80 \\
\hline Baler & 7.30 & 7.30 \\
\hline Bale collection/stacking & 3.30 & 3.30 \\
\hline Totals & 15.40 & 15.40 \\
\hline
\end{tabular}

\subsubsection{Storage}

The 2017 Design Case assumes that storage of corn stover and switchgrass will occur field side or at a similar unimproved storage site. Biomass storage systems in the 2017 Design Case seek to provide a low-cost, low-maintenance, moisture-tolerant solution that focuses on the predictability of dry matter losses and compositional changes to inform an active inventory management approach to large-scale, long-term storage.

\subsubsection{Biomass Storage Design Base}

The 2017 Design Case is based on material entering storage with $30 \%$ moisture. While it is recognized that this condition is not the norm for many areas and that storage performance will vary accordingly, use of this approach ensures the supply system will be capable of dealing with unstable, non-ideal feedstock. According to INL data shown in Figure 27, we assume that corn stover at 30\% moisture accumulates, at-worst, 12\% dry matter loss after about 150 days in storage (adjusted for time scale) if additional moisture is not inserted. This upper limit for dry matter loss was assumed for the entire year's lot of feedstock. 

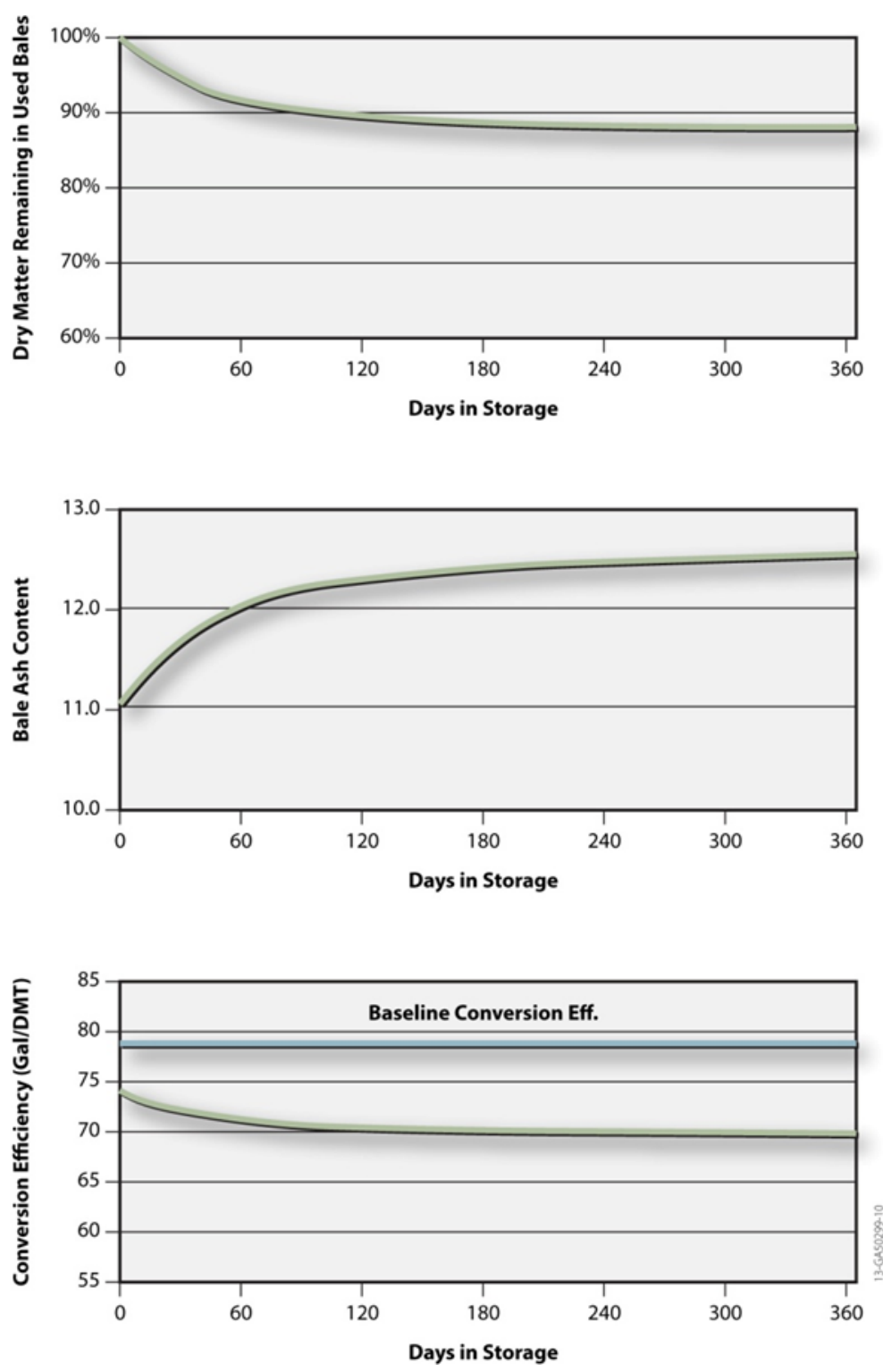

Figure 27. The impact of dry matter loss on bale ash content and final conversion efficiency (based on a $30 \%$ initial moisture and $12 \%$ ash).

As discussed in terms of the 2013 SOT, the passive loss of moisture during storage using conventional practice cannot be depended on as means to safely store wet feedstock. Therefore, storage practices developed by 2017 must be capable of limiting dry matter loss and its associated impact on convertibility, even when moisture contents entering storage are not favorable. To this end, the reduction of dry matter loss will be achieved through actively controlled improvements to storage in a way that moisture loss can be reliably achieved and/or oxygen availability can be limited in baled storage; both of which effectively limit microbial growth. Laboratory testing at INL has demonstrated that the availability of oxygen (while maintaining an aerobic storage environment) can effectively reduce the rates of dry matter loss in storage (Figure 28). These high-moisture corn stover samples (i.e., 50\% wet basis) demonstrate 
how oxygen limitation can extend the shelf life in aerobic storage. Ongoing research will determine how practical measures, such as increasing bale density, high-density stacking configurations, and tarping, can be used to limit oxygen availability and improve storage stability in high-moisture, baled, and bulk stored feedstocks.

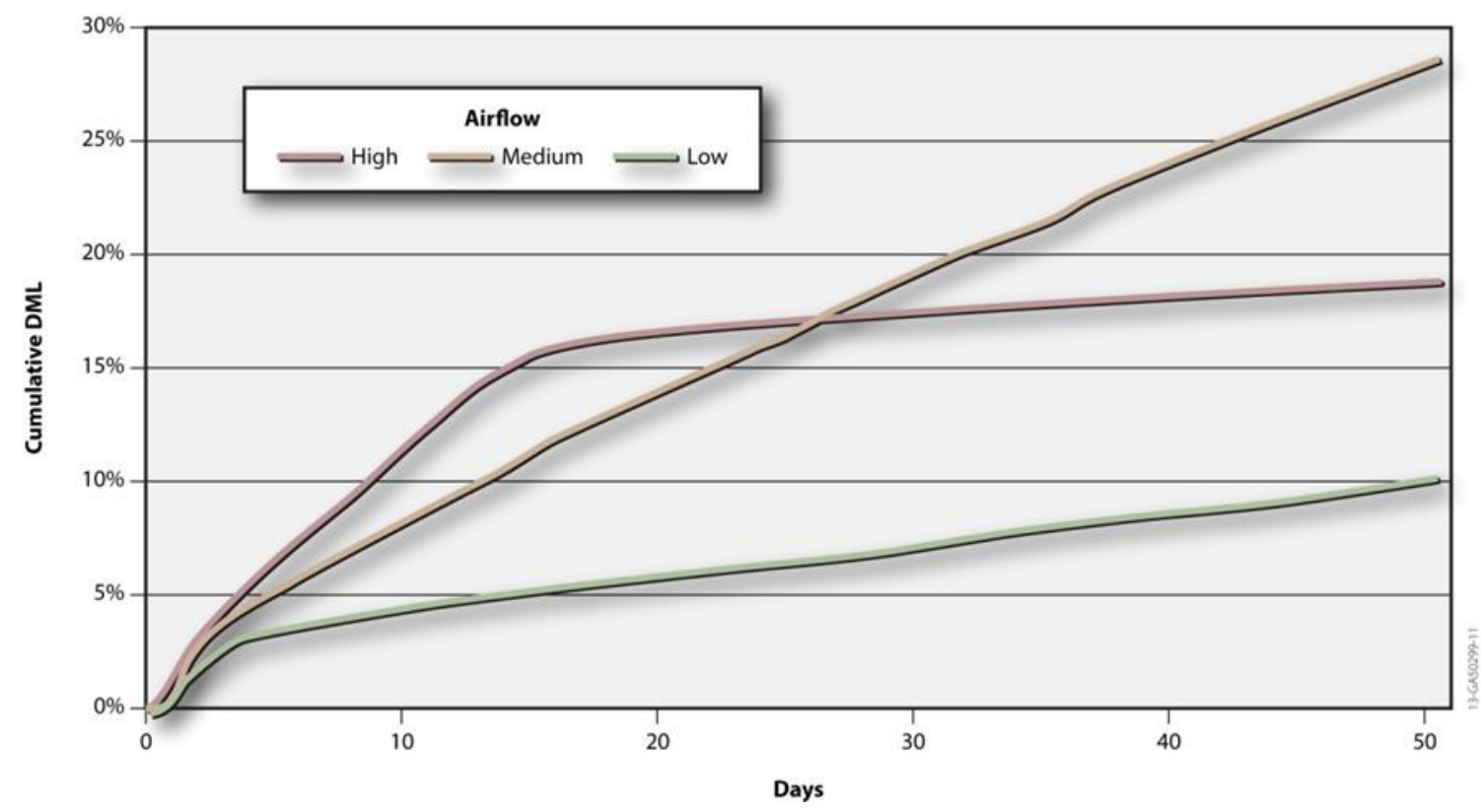

Figure 28. Dry matter loss of corn stover in the simulated storage conditions, with three air flows simulating three different oxygen availabilities.

The 2017 Design Case shifts the traditional focus of storage management away from a singular goal of minimizing dry matter loss to a more informed focus on the final material's convertibility. This approach allows the conversion yield, reasonably derived from stored biomass, to be assessed in addition to the mass loss incurred. The 2017 Design Case assumes that structural carbohydrates consumed during storage leave the remaining dry matter less convertible than the starting material. As an example of this effect, a hypothetical analysis of a storage scenario using the 2013 Base Case feedstock (30\% moisture and 12\% ash) was cast in terms of the existing biochemical ethanol conversion pathway ${ }^{46}$. Regardless of final product class (e.g., ethanol versus bio-based hydrocarbon fuels), it is assumed the decreased conversion performance due to degradation in storage will have comparable impacts on the feedstock supply system, with actual impacts dependent on product-specific conversion specifications. For the purpose of this analysis, calculation in terms of ethanol presents the opportunity for a direct comparison of feedstock performance and should not be inferred as yield goals for 2017 . The analysis shows a conversion efficiency drop to $70 \mathrm{gal} /$ dry $\mathrm{T}$, which is an $11 \%$ reduction compared to the baseline of $79 \mathrm{gal} /$ dry T (Figure 29). The analysis assumes that dry matter losses are confined to the nonash biomass fraction, dry matter loss occurs proportionally across all non-ash components, and for each $1 \%$ dry matter lost, there is a $0.25 \%$ decrease in conversion efficiency, which is defined as a reduction in final product yield. As a result, when dry matter loss is accumulated over time in storage (Figure 27, top), several important behaviors and interactions are occurring, primarily the relative ash content of the material is becoming enriched (Figure 27, middle), causing the 
carbohydrate fraction of the biomass to respectively diminish (deviance from carbohydrate quantity spec), and the conversion performance of the remaining biomass is being reduced (deviance from the carbohydrate quality spec; Figure 27, bottom). These actions impact replacement costs, operational costs, and disposal costs for the refinery because more biomass must to be procured (replacement costs), more biomass must be handled and treated throughout the conversion process (operational costs), and more waste is being generated (disposal costs). In the 2013 Base Case, where feedstock price is $\$ 121.60$ /dry T, these costs result in a total feedstock dockage of $\$ 18.93 /$ dry T, comprised of $\$ 12.48$ /dry T from feedstock replacement, $\$ 4.16 /$ dry $\mathrm{T}$ from operational costs, and $\$ 2.28 /$ dry $\mathrm{T}$ from disposal costs. Of these costs, dry matter loss is responsible for $\$ 6.10 /$ dry $\mathrm{T}$.

The technical targets for 2017 reduce this cost through decreases in dry matter loss (i.e., structural sugar quantity and quality preservation) and the ash entering storage. When the above simulation is applied to the 2017 Design Case specifications (i.e., 30\% moisture, $4.9 \%$ ash, annual dry matter loss of $7 \%$, and a $\$ 81.60 /$ dry $\mathrm{T}$ feedstock price), the dry matter loss results in a total convertibility dockage of $\$ 3 /$ dry $\mathrm{T}$ (Table 14). These reductions in storage-related losses will be achieved by 2017 through the minimization of microbial activity in storage; principally, through controlled limitation of moisture content and/or oxygen in stored herbaceous feedstock.

Table 14. Biomass storage design basis.

\begin{tabular}{|c|c|c|c|c|}
\hline & SOT & Target & SOT & Targets \\
\hline & \multicolumn{2}{|c|}{ Dry Matter Loss } & \multicolumn{2}{|c|}{ Convertibility $^{a}$} \\
\hline Corn Stover & $12 \%$ & $8 \%$ & $59 \mathrm{gal} / \mathrm{dry} \mathrm{T}$ & $79 \mathrm{gal} / \mathrm{dry} \mathrm{T}$ \\
\hline Switchgrass & $12 \%$ & $6 \%$ & $59 \mathrm{gal} / \mathrm{dry} \mathrm{T}$ & 79 gal/dry T \\
\hline
\end{tabular}

\section{Biomass Storage Cost Estimation}

Cost estimations for biomass storage were calculated based on the storage cover vendor's information and laboratory and field level experiments (Table 15).

Table 15. Field-side storage cost estimation.

\begin{tabular}{cccccc|}
\hline \multicolumn{2}{c}{ SOT $(2011$ \$/dry T) } & \multicolumn{3}{c|}{ Design Target $(2011$ \$/dry T) } \\
\hline Storage & Dockage & Total & Storage & Dockage & Total \\
$\mathbf{4 . 3 0}$ & 6.10 & 10.40 & 3.50 & 3.00 & 6.50
\end{tabular}

\subsubsection{Preprocessing}

Biomass preprocessing operations of the 2017 Design Case (Figure 29) differ substantially from the current state of technology, including improvements to size reduction (milling) and drying processes and the inclusion of new preprocessing operations (e.g., chemical preconversion and formulation) for ash reduction and feedstock blending. Biomass preprocessing begins with a 
coarse (i.e., Stage 1) size reduction to break the bale and facilitate the subsequent separations process. The next step is to separate the fractional material into two streams, one stream needing further grinding and the other stream that is at final size. The objective of biomass separations is to reduce the quantity of material that requires further preprocessing, differentiating among anatomical or size fractions based on size, material properties (e.g., moisture and density), and/or composition. In the 2017 Design Case, substantial cost savings in size reduction are realized by separating the fraction of the biomass that meets the particle size specification as it exits the Stage 1 size-reduction process, passing only the remaining over-sized materials on to the Stage 2 size-reduction process.

Separation/sorting of MSW is required to remove recyclables (e.g., metal, paper, and cardboard), contaminants (e.g., plastics and concrete), and other unusable fractions to isolate only those fractions that meet the cost and quality requirements for biofuel feedstocks. In the 2017 Design Case, MSW is sorted to supply only yard and construction/demolition waste, which consists mainly of wood waste (e.g., tree trimmings and lumber), as a feedstock to be blended with corn stover and switchgrass. The ash content of these select MSW fractions is estimated to be about $10 \%$. Chemical preconversion will be necessary for additional ash reduction (see Appendix B). Following final milling of over-sized materials to the particle-size specification (i.e., 1/4-in. minus), feedstocks are pelletized.

The 2017 Design Case incorporates many improvements in preprocessing, including fractional milling, chemical preconversion, high-moisture densification, and formulation/blending. Figure 29 outlines the material flow given for these improvements.

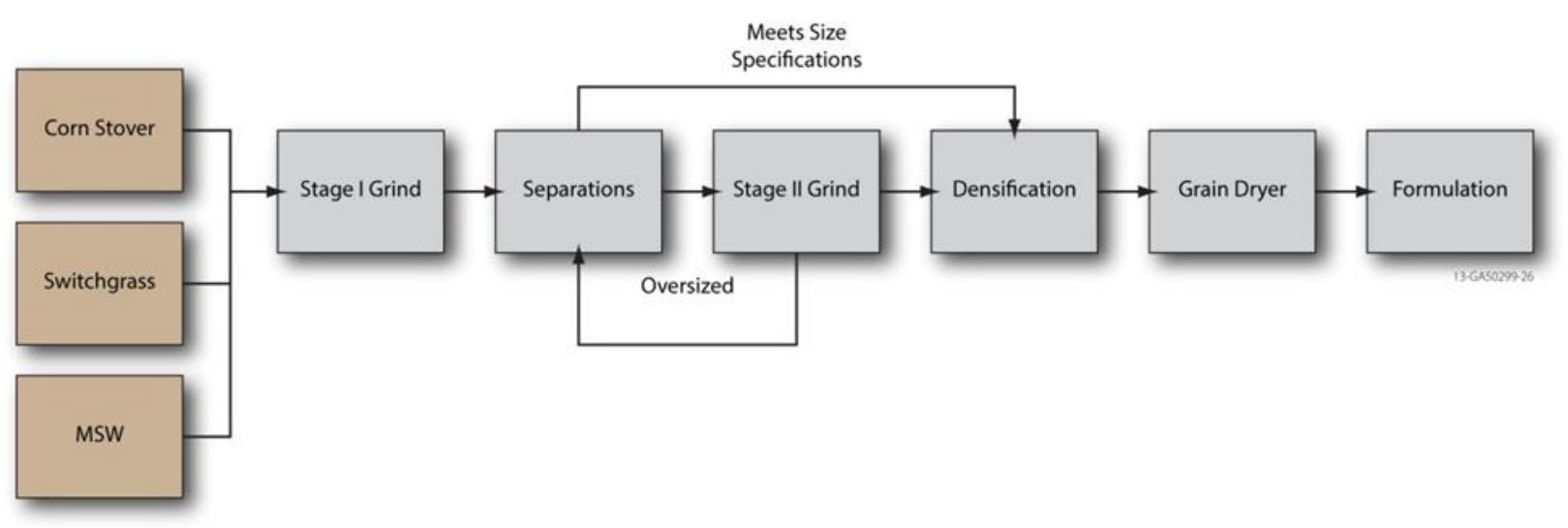

Figure 29. Material flow in the 2017 Design Case that incorporates many improvements in preprocessing, including fractional milling, chemical preconversion, high-moisture densification, and formulation/blending.

The logistics of a blended feedstock scenario are certainly more complex than a single-feedstock scenario. The 2017 Design Case assumes that preprocessing of MSW will occur at a preprocessing depot located at the source landfill or refuse transfer station, and MSW pellets will be shipped from the depot to the blending depot located within proximity of the biorefinery. Corn stover and switchgrass that is formatted in large square bales will be delivered to the blending depot, where they will be processed into pellets. Corn stover, switchgrass, and MSW 
pellets will be queued up in blending bunkers or silos. The pellets of the three blendstocks (i.e., corn stover, switchgrass, and MSW) are then metered from the blending bunkers in the ratios required of the blended feedstock and are conveyed from the preprocessing facility/depot to the conversion facility.

\subsubsection{Size Reduction}

For the 2017 Design Case, a geometric mean particle size of $1 / 4-$ in. is the target size specification optimal for densification. Particle size after grinding is dictated by a number of factors, including biomass physical and material properties, process variable of the comminution system, shear and impact forces imparted by the comminution system, and the size opening of the screen used to retain material in the system until the material is sufficiently processed to pass through the screen.

Hammer mills generally are considered the current state of technology for biomass comminution due to their high throughputs and versatility in processing a wide range of materials. As a general rule of thumb, the geometric mean particle size achieved by hammer milling typically is an order of magnitude smaller than the screen size opening

The fractional milling design basis is summarized in Table 16. Preprocessing starts with an initial (Stage 1) coarse size reduction using a 400-hp horizontal grinder configured with a 6-in. screen. Upon exiting the first-stage grinder, the coarse-ground material passes through a separator that is configured with a 1/4-in. screen. The fraction that meets the size specification will pass through the screen and move onto densification, while the fraction that is retained on the screen will be conveyed into the second-stage size-reduction process for final milling to the particle size specification. The fractional milling process will reduce the total effective energy consumption for biomass size reduction by about 60 and $70 \%$ for dry $(15 \%)$ and wet $(30 \%)$ biomass, respectively. Note that this calculation is based on the effective energy consumption for second-stage comminution (see footnote to Table 16). 
Table 16. Size-reduction design basis.

\begin{tabular}{|c|c|c|c|c|}
\hline & \multicolumn{2}{|c|}{2013 SOT } & \multicolumn{2}{|c|}{2017 Target } \\
\hline & Stage 1 & Stage 2 & Stage 1 & Stage 2 \\
\hline Screen size & 2 in. & 1 in. & 6 in. & 1 in. \\
\hline $\begin{array}{l}\text { Comminution energy at } 15 \% \text { moisture } \\
(\mathrm{kWhr} / \mathrm{dry} \mathrm{T})\end{array}$ & 39 & 21 & 10 & $21 *$ \\
\hline $\begin{array}{l}\text { Comminution energy at } 30 \% \\
\text { moisture(kWhr/dry } \mathrm{T})\end{array}$ & 40 & 60 & 15 & $21 *$ \\
\hline $\begin{array}{l}\text { Separations at } 15 \% \text { moisture (percent } \\
\text { passing } 1 / 4 \text {-in. screen) }\end{array}$ & 100 & 100 & 100 & 55 \\
\hline $\begin{array}{l}\text { Separations at } 15 \% \text { moisture (percent } \\
\text { passing } 1 / 4 \text {-in. screen) }\end{array}$ & 100 & 100 & 100 & 55 \\
\hline
\end{tabular}

\section{Fractional Milling Cost Estimation}

Fractional milling cost estimation is based on vendor-supplied information and equipment performance from typical machine performance and process demonstration unit data (Table 17).

Table 17. Fractional milling cost estimates

\begin{tabular}{|lcc|}
\hline & $\begin{array}{c}\text { 2013 SOT } \\
(2011 \$ / \text { dry T })\end{array}$ & $\begin{array}{c}\text { 2017 Target } \\
(2011 \text { \$/dry T })\end{array}$ \\
\hline Grinder 1 & Total & Total \\
\hline Separations & 16.80 & 5.10 \\
\hline Grinder 2 & NA & 5.00 \\
\hline Total & 11.60 & 2.40 \\
\hline
\end{tabular}

\subsubsection{Drying and Densification}

\section{Cost Estimation for High-Moisture Densification}

The reduction in drying energy is the key advantage of this approach. First, the process uses the heat generated in the pellet die to partially dry the material. Second, drying the pellets offer cost and energy advantages over drying loose, bulk biomass. Loose biomass typically is dried in a concurrent flow rotary dryer. Rotary biomass dryers typically operate at temperatures of about 150 to $160^{\circ} \mathrm{C}$, have greater particulate emissions, greater volatile organic compound emissions, greater fire hazard, a large footprint, and often have difficulty in controlling the material moisture. With the increased density, the reduced tendency for material to become entrained in the air flow, and the increased heat transfer coefficients compared to loose biomass, more efficient drying technologies options are available for drying pellets. A cross-flow dryer (common in grain drying) operates at temperatures less than $100^{\circ} \mathrm{C}$, reduces the particulate and volatile organic compound emissions, and will have better temperature distribution. A 
comparison of pellet properties and energy balances for conventional and high-moisture pelletization processes is given in Table 18. The table shows 2017 Design Case targets to achieve a 40 to $50 \%$ reduction in the total pelletization and drying energy.

Table 18. Drying and densification design basis

\begin{tabular}{lcc} 
Moisture Properties & $2013 \mathrm{SOT}$ & 2017 Target \\
\hline Infeed Moisture & $30 \%$ & $30 \%$ \\
Dryer Moisture Reduction & $18 \%$ & $11 \%$ \\
Densification Moisture Reduction & $3 \%$ & $10 \%$ \\
\hline Final Pellet Moisture & $9 \%$ & $9 \%$ \\
Densification Energy & $75 \mathrm{kWhr} / \mathrm{dry} \mathrm{T}$ & $50 \mathrm{kWhr} / \mathrm{dry} \mathrm{T}$ \\
\hline $\begin{array}{l}\text { Drying Energy } \\
\text { Pellet Properties }\end{array}$ & $350 \mathrm{kWhr} / \mathrm{ton}$ & $100 \mathrm{kWhr} / \mathrm{ton}$ \\
\hline $\begin{array}{l}\text { Unit Density } \\
\text { Bulk Density }\end{array}$ & $70 \mathrm{lb} / \mathrm{ft}^{3}$ & \\
Durability & $40 \mathrm{lb} / \mathrm{ft}^{3}$ & $65 \mathrm{lb} / \mathrm{ft}^{3}$ \\
\hline
\end{tabular}

The cost of densification was estimated using vendor-supplied information and the capacity and energy assumptions shown in Table 19. Rotary drying costs associated with the 2013 SOT were based on data supplied by Anco-Eaglin, Inc. As described above, because of the similarity of pellets and grain, grain drying technology is the basis of the 2017 Design Case. Accordingly, grain drying costs also the source of the pellet drying cost estimate. Using a grain drying calculator found at Iowa State ${ }^{49}$, we estimate the cost of drying grain of a similar moisture content to be $\$ 10$ to $\$ 14 /$ ton. Estimated pellet drying costs were reduced from these values because we assume that the porous nature of pellets and less structural heterogeneities in pellets will promote more rapid and uniform drying compared to grain that has the outer pericarp layer that limits moisture transfer. 
Table 19. Drying and densification cost estimates

\begin{tabular}{|ccl|} 
& $\begin{array}{l}\text { 2013 SOT } \\
(2011 \text { \$/dry T) }\end{array}$ & $\begin{array}{l}\text { 2017 Target }(2011 \\
\text { \$/dry T) }\end{array}$ \\
\hline Drying & Total & Total \\
\hline Densification & 15.20 & 5.60 \\
Totals & 7.70 & 4.40 \\
\hline
\end{tabular}

\subsubsection{Formulation/Blending}

\section{Overview}

Feedstock formulation is not a new concept in many market sectors. For example, different grades of coal are blended to reduce sulfur and nitrogen contents for power generation ${ }^{14}$, grain is blended at elevators to adjust moisture content ${ }^{13}$, animal feeds are blended to balance nutrient content ${ }^{16}$, and high-ash biomass sources are mixed with low-ash coal to allow their use in biopower $^{17}$. However, blending/formulation is not part of the baseline design.

\section{Formulation Design Basis}

To meet feedstock specifications required for various conversion pathways, formulation of specific mixtures of feedstocks will likely be required. Examples include mixing high and lowcost feedstocks to meet cost targets, mixing high and low-ash feedstocks to meet an ash target, mixing of high and low-carbohydrate feedstocks to meet a yield target, and mixing easily and poorly reactive feedstocks to meet a convertibility target. An example of blending to meet an ash and moisture specification is shown in Table 20.

Table 20. Feedstock formulation/blending of ash and moisture contents*.

\begin{tabular}{|c|c|c|c|c|c|}
\hline $\begin{array}{l}\text { Content Delivered to } \\
\text { Biorefinery Infeed }\end{array}$ & $\begin{array}{l}\text { Single-pass } \\
\text { cornstover( }(35 \%)\end{array}$ & $\begin{array}{l}\text { Multi-pass } \\
\text { cornstover }(25 \%)\end{array}$ & $\begin{array}{l}\text { Switchgrass } \\
(35 \%)\end{array}$ & $\begin{array}{c}\text { MSW } \\
(5 \%)\end{array}$ & Final Blend \\
\hline Ash content (wt. \%) & 3.5 & 7 & 4 & 10 & 4.9 \\
\hline $\begin{array}{l}\text { Moisture content (\%, wet } \\
\text { basis) }\end{array}$ & 9 & 9 & 9 & 9 & 9 \\
\hline $\begin{array}{l}\text { Carbohydrate content (wt. } \\
\% \text { ) }\end{array}$ & 64 & 57 & 57 & 57 & 59 \\
\hline
\end{tabular}

Assumptions for the formulation design basis are as follows:

- Blended feedstocks will be selected and developed to achieve conversion yield specifications. It is currently unknown how blended feedstocks will perform in the conversion pathways. The simplest assumption is that the performance of the blended feedstocks would be the sum of performances of each individual component. However, two, small-scale studies demonstrated that the performance of blended feedstocks ranged from under to over performance, depending on the conditions assessed. In the first study ${ }^{50}$, a blend of wheat straw, barley straw, hardwood, and softwood subjected to three different types of 
pretreatments: dilute acid, lime, and soaking in aqueous ammonia was examined. After pretreatment, the feedstocks were hydrolyzed using commercial cellulose enzymes ${ }^{51}$ and sugar yields were measured. Ethanol yields also were determined using simultaneous saccharification and fermentation ${ }^{52}$.

- For the dilute acid and soaking in aqueous ammonia treatments, the yields of C6 sugars were lower than would be predicted by simple summation, while the C6 sugar yield was slightly higher than predicted for the lime treatment. However, the opposite trends were observed for ethanol production, with higher ethanol production for dilute acid and soaking in aqueous ammonia and lower production for lime treatment. It is not clear from the report whether or not these differences were statistically significant. It also was shown that yields of both C6 sugars and ethanol were lower than predicted for non-optimized pretreatments. This may indicate that the pretreatment has to be optimized for the most recalcitrant component, which may lead to formation of sugar degradation products and fermentation inhibitors. In the second study, ${ }^{53}$ examined a mixture of corn stover, switchgrass, eucalyptus, and lodgepole pine. This mixture was pretreated with an ionic liquid (i.e., 1-ethyl-3-methylimidazolium acetate) and the resulting sugars measured. The mixed feedstock released more glucose than would be expected from the sum of the individual feedstocks.

- Individual feedstocks will be pelleted at depots for shipment to biorefineries. At the biorefinery, the pelleted feedstocks will be unloaded and conveyed into individual bunkers for storage. Pellets of the different blendstocks will be metered out into the bunkers in the ratios required of the blends, crushed (using a pellet crusher), and then mixed prior to insertion into the conversion process.

- Material will be metered from individual bunkers onto a conveyer and will be thoroughly homogenized during this process with no segregation. Mixing of solids occurs in many industries and is often problematic when solids of varying density, shape, and size are blended. This often leads to segregation, either during the mixing or while being transported to its destination. Mixing of solids is considered a trial-and-error process due to these issues.

- The expected unit operations for formulation are shown in Table 21.

Table 21. Feedstock formulation design basis.

\begin{tabular}{lcc|}
\hline 2013 SOT $(2011 \$)$ & \multicolumn{2}{c}{ Operating Parameters } \\
\hline Pellet Pulverizer & 100 dry T/hour & Horsepower \\
\hline Bulk Storage with Hopper & 30 dry T/hour & $200 \mathrm{HP}$ \\
\hline Conveying/Mixing System & 30 dry T/hour & $30 \mathrm{HP}$ \\
\hline
\end{tabular}

Research currently is ongoing at INL to examine the compatibility of various feedstocks in formulated blends, with an initial focus on the reactivity of blends versus the individual feedstocks. Blends will be developed for several regions of the United States using the least-cost formulation model as a starting point and will incorporate feedstocks with varying levels of reactivity (e.g., herbaceous, woody, and MSW). Reactivity for the fermentation pathway will be investigated first, with expansion into the other DOE conversion pathways in later fiscal years. 
Reactivity for the fermentation conversion pathway will be measured as production of sugars using dilute acid pretreatment followed by enzymatic hydrolysis. Production of sugar decomposition products and other inhibitors also will be monitored. Hydrolysis conditions will be optimized for each feedstock and then each of the optimum conditions used on the formulated feedstock. Research is planned to examine mixing issues associated with blended feedstocks. A survey of current state-of-the-art mixing technologies will be conducted, and those technologies relevant to feedstocks will be further examined to determine the best technology to ensure thorough homogenization without segregation.

While the costs for preprocessing of herbaceous feedstocks (e.g., grinding, chemical preconversion, pelleting, and drying) are addressed in other parts of the 2017 Design Case, MSW will require a different set of preprocessing options to produce a stable, high-quality feedstock.

\section{Cost Estimation for Formulation}

Formulation cost estimation was based on existing technology, vendor-supplied information and equipment performance (Table 22).

Table 22. Formulation cost estimation.

\begin{tabular}{|lc|}
\hline & 2017 Target $(2011 \$ /$ dry $\mathrm{T})$ \\
\hline Pellet pulverizer & Total \\
\hline Bulk storage with hopper & 0.10 \\
\hline Conveying/Mixing system & 0.60 \\
Totals & 1.90 \\
\hline
\end{tabular}

\subsubsection{Transportation and Handling}

The 2017 Design Case includes formulation and densification to meet feedstock specifications and costs targets. Both of these active processes will improve feedstock handling operations through active controls. Given formulation and the specific quantities of individual feedstocks required, the average transportation distance (and even mechanism) will change based on feedstock type. In the 2017 Design Case, corn stover will be trucked from a local draw radius of about 25 miles (compared to 35 miles) while switchgrass will be trucked 15 miles. MSW will need to be transported from a larger metropolitan area to obtain the required quantities; therefore, it will be transported by rail (either by unit train or single car) from as far as 200 miles away. Corn stover and switchgrass will be loaded and unloaded at each location using a telehandler capable of moving $12-\mathrm{lb} / \mathrm{ft}^{3}$ bales at 30 and $20 \%$ moisture contents respectively. A $53-\mathrm{ft}$ trailer and 800,000-GVW limits were assumed in all trucking operations. Transportation for corn stover and switchgrass will occur from a field side stack to a densification facility completely separate from the conversion location, but is within a minimal conveyor distance. MSW transportation will occur from the waste transfer station to a densification facility. Further transportation and handling assumptions are given as follows: 
- At 30\% moisture, transportation of cornstover continues to be volume limited due to low densities $\left(12 \mathrm{lb} / \mathrm{ft}^{3}\right)$

- At $20 \%$ moisture, transportation of switchgrass continues to be volume limited due to low densities $\left(12 \mathrm{lb} / \mathrm{ft}^{3}\right)$

- There will be insignificant material losses throughout transportation and handling.

- Densification will increase material uniformity and flowability.

\subsubsection{Cost Estimation for Transportation}

The cost estimation for transportation and handling was based on vendor-supplied information and equipment performance from typical machines (Table 23).Rail transportation costs were based on work from Searcy using a jumbo hopper car $^{54}$ adjusted for U.S. conditions.

Table 23. Transportation cost estimates.

\begin{tabular}{|ccc|} 
& $\begin{array}{c}2013 \text { SOT } \\
(2011 \text { \$/dry T })\end{array}$ & $\begin{array}{c}2017 \text { Target } \\
(2011 \text { \$/dry T })\end{array}$ \\
\hline Truck & 11.50 & Total \\
\hline Rail* & 0.00 & 8.30 \\
\hline $\begin{array}{l}\text { *For specific } \\
\text { quantity. }\end{array}$ & feedstocks only to obtain & required \\
\hline
\end{tabular}

\subsection{Life cycle Assessment:}

This section uses a life-cycle assessment (LCA) to evaluate the energy input and GHG emissions for Biological Conversion of Sugars to Hydrocarbons. LCA considered the unit processes involved in the biomass logistics. Table 24 shows the energy consumption and Table 25 shows GHG contribution for Biochemical conversion supply chain design. 
Table 24. Energy consumption for Biochemical conversion supply chain design.

\begin{tabular}{|c|c|c|c|c|c|}
\hline Process Element & $\begin{array}{l}\text { Single-pass } \\
\text { Corn Stover }\end{array}$ & $\begin{array}{l}\text { Multi-pass } \\
\text { Corn Stover }\end{array}$ & Switchgrass & MSW & Blend \\
\hline Formulation Contribution & $35 \%$ & $25 \%$ & $35 \%$ & $5 \%$ & - \\
\hline $\begin{array}{l}\text { Harvest and collection } \\
\text { (MBTU/dry T) }\end{array}$ & 108.69 & 204.18 & 122.85 & - & 132.08 \\
\hline Transportation (MBTU/ dry T) & 142.08 & 142.08 & 99.35 & 549.43 & 147.49 \\
\hline Preprocessing (MBTU/dry T) & 315.82 & 315.82 & 285.83 & 421.25 & 310.60 \\
\hline Storage (MBTU/dry T) & 21.83 & 21.83 & 21.83 & 21.83 & 21.83 \\
\hline Handling (MBTU/dry T) & 41.90 & 41.90 & 41.90 & 41.90 & 41.90 \\
\hline $\begin{array}{l}\text { Total Energy Consumption Cost } \\
\text { (MBTU/dry T) }\end{array}$ & 630.32 & 725.81 & $\mathbf{5 7 1 . 7 6}$ & 1034.41 & 653.90 \\
\hline
\end{tabular}

Table 25. GHG contribution for biochemical conversion supply chain design.

\begin{tabular}{|c|c|}
\hline Process Element & \\
\hline Formulation Contribution & - \\
\hline $\begin{array}{l}\text { Harvest and collection } \\
\text { GHGs }\left(\mathrm{Kg} \mathrm{CO}_{2} \mathrm{e} / \text { dry T) }\right.\end{array}$ & 10.90 \\
\hline Transportation GHGs ( $\mathrm{Kg} \mathrm{CO}_{2} \mathrm{e} /$ dry T) & 8.62 \\
\hline Preprocessing GHGs $\left(\mathrm{Kg} \mathrm{CO}_{2} \mathrm{e} /\right.$ dry $\left.\mathrm{T}\right)$ & 27.02 \\
\hline Storage GHGs $\left(\mathrm{Kg} \mathrm{CO}_{2} \mathrm{e} /\right.$ dry $\left.\mathrm{T}\right)$ & 6.42 \\
\hline Handling GHGs ( $\left.\mathrm{Kg} \mathrm{CO}_{2} \mathrm{e} / \operatorname{dry} \mathrm{T}\right)$ & 6.41 \\
\hline Total GHGs $\left(\mathbf{K g ~ C O} \mathbf{C O}_{2} \mathbf{e} / \mathbf{d r y} \mathbf{T}\right)$ & 59.40 \\
\hline
\end{tabular}




\section{Conversion of Lignocellulosic Biomass to Hydrocarbon Fuels: Fast Pyrolysis and Hydrotreating Bio-Oil Pathway}

This section is intended to couple with the Pacific Northwest National Laboratory's (PNNL's) hydrocarbon design report, "Process Design and Economics for the Conversion of Lignocellulosic Biomass to Hydrocarbon Fuels: Fast Pyrolysis and Hydrotreating Bio-Oil Pathway" 55 that describes a viable route from biomass to hydrocarbon fuels. The assumptions of scale and feedstock quality requirements are consistent with the design case assumptions used by PNNL in their report and techno economic assessments. This design does not consider the different requirements and nuances of other thermochemical conversion processes or other hydrocarbon pathways.

\subsection{Summary}

This report establishes a plausible case for achieving the 2017 Design Case for Fast Pyrolysis conversion to bio-oils cost goals of delivering a biomass feedstock to the conversion facility at a cost of $\$ 80 /$ dry T (Table 26). The least-cost formulation approach (Appendix B) illustrates the importance of cost estimates for determining the total cost of feedstock to a biorefinery, including grower payment (access costs), logistics costs, and quality/dockage cost. It also illustrates the importance of refining and updating these costs as analyses and data improve to better inform the estimates. The following conclusions are presented to document the specific areas that require additional attention to further strengthen and support the feedstock design detailed in this report. 
Table 26. Thermochemical feedstock design cost analysis for 2017.

\begin{tabular}{|c|c|c|c|c|c|}
\hline Cost Element & Pulpwood & Wood Residues & Switchgrass & $\begin{array}{c}\text { Construction and } \\
\text { Demolition Waste } \\
\text { (C\&D) }\end{array}$ & Blend \\
\hline Formulation Contribution & $45 \%$ & $32 \%$ & $3 \%$ & $20 \%$ & - \\
\hline $\begin{array}{l}\text { Grower payment/access } \\
\text { cost }\end{array}$ & 25.00 & 26.35 & 19.67 & 8.15 & 21.90 \\
\hline $\begin{array}{l}\text { Harvest and collection } \\
(\$ / \text { dry } T)\end{array}$ & 22.24 & 0 & 15.41 & - & 10.47 \\
\hline $\begin{array}{l}\text { Landing Preprocessing/ } \\
\text { Sorting (\$/dry T) }\end{array}$ & 12.17 & 8.73 & 0 & 9.85 & 10.24 \\
\hline Transportation (\$/dry T) & 10.89 & 3.33 & 4.59 & 6.87 & 7.48 \\
\hline Preprocessing (\$/dry T) & 23.97 & 23.97 & 19.7 & 28.12 & 24.67 \\
\hline Storage (\$/dry T) & 3.23 & 3.23 & 5.59 & 3.23 & 3.30 \\
\hline Handling (\$/dry T) & 1.90 & 1.90 & 1.90 & 1.90 & 1.90 \\
\hline $\begin{array}{l}\text { Total Delivered Feedstock } \\
\text { Cost }(\$ / \text { dry T) }\end{array}$ & 99.49 & 67.51 & 66.86 & 58.12 & 80.00 \\
\hline \multicolumn{6}{|c|}{ Delivered Feedstock Specifications } \\
\hline Ash content (wt. \%) & 0.5 & 1.0 & 4.0 & 1.0 & $<1 \%$ \\
\hline $\begin{array}{l}\text { Moisture content (\%, wet } \\
\text { basis) }\end{array}$ & 9 & 9 & 9 & 9 & 9 \\
\hline HHV (BTU/lb) & 8824 & 9444 & 7557 & 8824 & 8984 \\
\hline LHV (BTU/lb) & 7255 & 7616 & 6155 & 7255 & 7337 \\
\hline
\end{tabular}

Continued refinements of the biomass supply curves to represent the latest estimates for biomass grower payment are needed to support the least-cost formulation approach. Ultimately, translating The Billion Ton Update ${ }^{5}$ data from farm gate price to grower payment is necessary to establish better grower payment estimates. The grower payment estimates included in this report were calculated by subtracting our harvest and collection costs from the farm gate price.

Logistics costs are based on actual field trial data but do not include the cost of various business elements, such as profit margins for transportation, depots and field agents that would be involved throughout a biomass feedstock supply chain. This would increase the overall cost of the supply system than is demonstrated in this report. This was of little consequence to the 2012 Conventional Design Case target that intentionally focused only on logistics costs. The 2017 Design Case, on the other hand, is meant to encompass total delivered feedstocks costs. Further, the complexity of a blended feedstock approach may introduce multiple business elements into the supply chain; therefore, it is important that logistics costs be updated to include the true cost of these business elements, including a return on investment.

As the biomass logistic systems become more complex, especially with the introduction of new technologies (e.g., chemical preconversion), it may be prudent to differentiate between the current state-of-technology costs and the projected costs of mature technology ( ${ }^{\text {th }}$ plant costs) to 
be consistent with conversion platform terminology. This was not an issue with conventional feedstock designs that were intrinsically tied to current SOT; however, for technology maturation, cost reductions may be worth considering for advanced feedstock designs.

Admittedly, it also is necessary to tighten the design and cost estimates around formulation and the engineering systems for crushing the pellets and blending prior to insertion into the conversion process. A better understanding of C\&D availability, cost, and conversion performance is needed to solidify its position in the 2017 Design Case. Likewise, the viability of blended feedstocks as a whole depends on their conversion performance. DOE Bioenergy Technology Office funded research is investigating the conversion performance of blends (including C\&D blends) and evaluating the compatibilities and incompatibilities of blendstocks. The results of this research are critical to further development of blended feedstocks.

\subsection{Feedstock Composition (In-feed quality specifications)}

The dry basis elemental composition of the feedstock, shown in Error! Reference source not found., is identical to previous NREL and PNNL design reports ${ }^{6}$. The composition was originally assumed to come from pulpwood. Recent feedstock logistics work suggests the use of blended material may be required to meet a cost target of $\$ 80 /$ dry US ton while still meeting these specifications. For the purpose of this report, it is assumed that any blended material provided to meet this feedstock elemental composition will not adversely affect fast pyrolysis conversion efficiencies. Ongoing studies being conducted jointly by INL, NREL and PNNL will provide experimental evidence of the impact of blended feedstocks on fast pyrolysis and gasification processes. Future TEA will be modified to reflect conversion impacts inferred from such studies. As has been described in prior conversion design reports, the feedstock composition plays a critical role on overall process design and economics, primarily with respect to high and low heating values, carbon as well as high sensitivity to impurity components such as ash and metals in this design. The blended uniform-format feedstock composition assumed here for purposes of future design case targets is shown below, with supporting details described in the 2011 ethanol report ${ }^{46}$. Also consistent with prior design cases, the moisture content for the delivered feedstock is $10 \%$ or less. 
Table 27. Delivered woody feedstock composition and processing assumptions for the fast pyrolysis and hydrotreating design report ${ }^{6}$.

\begin{tabular}{|lc|}
\hline Component & $\begin{array}{c}\text { Composition } \\
\text { (dry wt. \%) }\end{array}$ \\
\hline Carbon & 50.94 \\
\hline Hydrogen & 6.04 \\
\hline Nitrogen & 0.17 \\
\hline Sulfur & 0.03 \\
\hline Oxygen & 41.90 \\
\hline Ash & $0.90-1.0$ \\
\hline Heating Value (Btu/lb) & $8,601 \mathrm{HHV}$ \\
\hline Moisture (Bulk Wt. \%) & $7,996 \mathrm{LHV}$ \\
\hline Particle Size (inch) & 10.0 \\
\hline
\end{tabular}

\subsection{Feedstock Selection Cost Estimation}

Expansion beyond highly productive resource areas has significant implications to the feedstock supply chain. Sparse areas, whether due to reduced yields and/or higher dispersion, typically increase feedstock logistics costs. Higher harvest and collection costs are incurred due to the need to spread machinery ownership costs over fewer tons of biomass or the need to cover more acres for the same quantity of biomass. Additionally, lower resource yields increase the supply radius and biomass transportation distances. Under the 2012 Conventional Design, higher yield areas allow refinery to be selective on the resource that they access.

Consider, for example, the scenarios depicted in Figure 30. This resource map illustrates a county-level resource assessment of pulpwood farm gate at $\$ 60 /$ dry T prices (this includes grower payment, harvest, collection, and chipping costs). Farm gate price data were extracted from The Billion Ton Update (BT2) ${ }^{5}$ data supplied from Oak Ridge National Laboratory. It should be noted that while the data is reported at a county-level, the data should be applied at the wood shed (typically much larger area than a county) level because it was derived from the U.S. Forest Service Forest Inventory and Assessment Data (FIA) ${ }^{56}$, and does not equate to county levels accurately. The FIA is a woodshed level assessment and therefore to use the data correctly it is necessary to combine multiple counties.

The cost competitiveness of the 2012 Conventional Design was demonstrated in the scenario located in southern Alabama, a high biomass yielding area ${ }^{3}$. We further suggest, based on the consistency of farm gate (i.e., landing) prices shown in this map, that the 2012 Conventional Design can be deployed cost effectively in South Carolina. Commercial readiness of conventional supply systems ultimately will be demonstrated by commercial-scale cellulosic ethanol plants opening in these areas in the near future. 


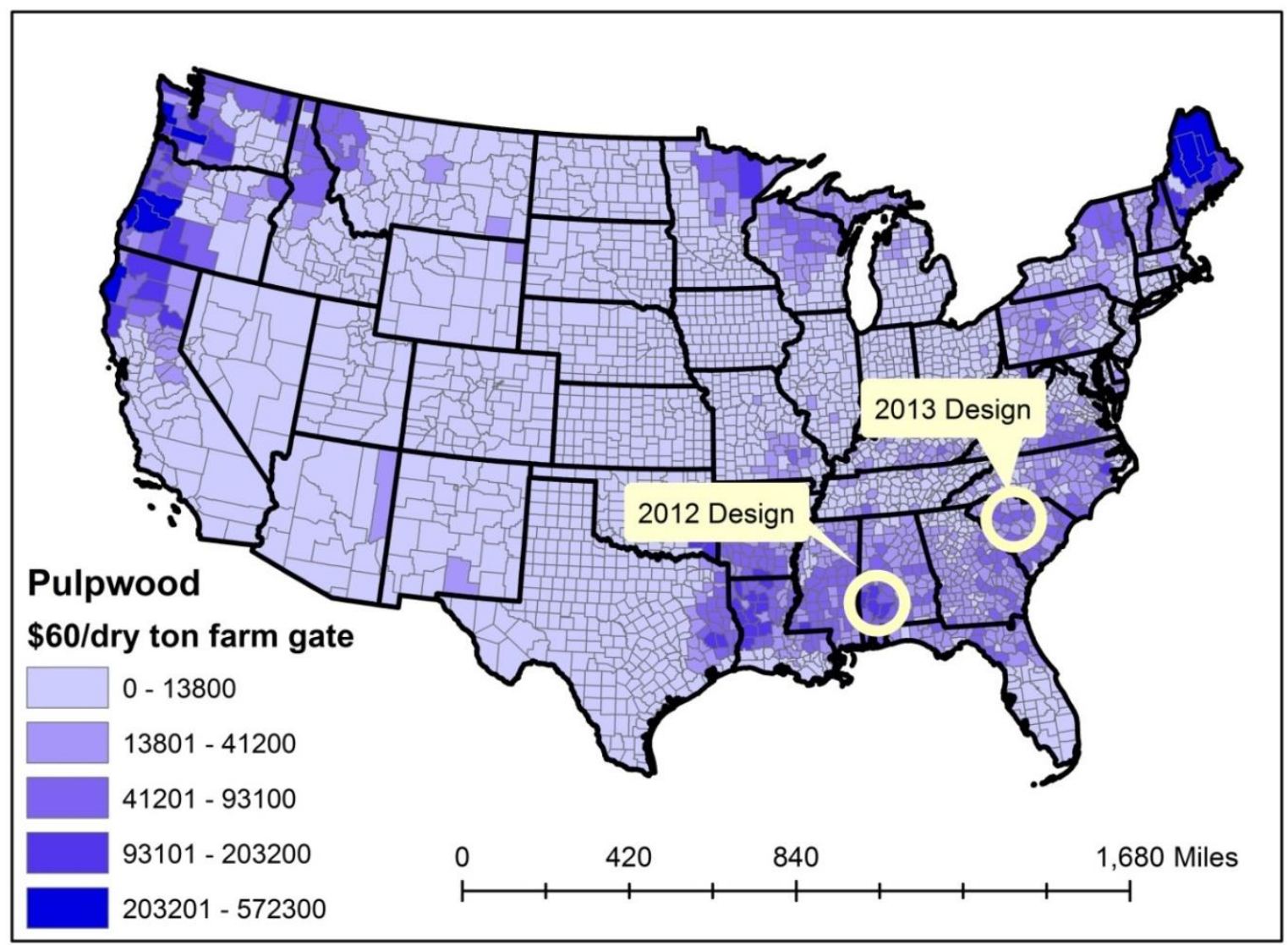

Figure 30. Total tons per county of available pulpwood at $\$ 60 /$ dry $\mathrm{T}$ farm gate price. Yellow circles show areas represented in the 2012 Conventional Design and the Relocated (2013) Design Case ${ }^{5}$.

Access costs are calculated from the grower payment cost curves shown in Figure 6, which are derived from historical prices. The 2017 Design Case basis discussion presented above provided the least-cost formulation approach for reducing access costs by accessing multiple feedstocks. With this approach, reduced quantities of each feedstock allows us to stay lower on the supply curve than if we had to supply the entire refinery with any single feedstock. The impact of this approach is shown in Table 28. The 2013 State of Technology assumes a 100\% supply of pulpwood of 909,100 dry $\mathrm{T}$ at an estimated $\$ 60 /$ dry $\mathrm{T}$ farm gate or a $\$ 25 /$ dry $\mathrm{T}$ access cost. In comparison, the 2017 Design Case blend of 45\% pulpwood, 32\% wood residues, 20\% C \& D waste, and 3\% switchgrass results in a weighted average feedstock cost that is nearly $15 \%$ lower than the access cost of pulpwood alone. 
Table 28. Resource access cost estimate (U.S. DOE $2011^{5}$ and INL MSW Data).

\begin{tabular}{lcccc} 
& \multicolumn{2}{c}{ 2013 SOT } & \multicolumn{2}{c}{ 2017 Target } \\
& $\begin{array}{c}\text { Access Cost } \\
(2011 \text { \$/dry T) }\end{array}$ & Tons & $\begin{array}{c}\text { Access Cost } \\
(2011 \text { \$/dry T })\end{array}$ & Tons \\
\hline Pulpwood & 25.00 & $909,100^{*}$ & 25.00 & $425,700^{*}$ \\
Wood Residues & NA & NA & 26.35 & $412,800^{* *}$ \\
Switchgrass & NA & NA & 19.67 & 25,800 \\
C\&D & NA & NA & 8.15 & 172,000 \\
Totals & 25.00 & NA & 21.90 & $1,036,300$
\end{tabular}

*assumes $10 \%$ loss of material to debark/delimb ${ }^{18}$.

**assumes $40 \%$ loss of material to clean up residues ${ }^{19}$.

\subsection{Quality Specification and Design Assumptions}

The 2012 Conventional Design focused on conventional woody harvest operations (i.e., felling and/ or skidding operations are separate from landing preprocessing operations). In addition to including switchgrass into the feedstock blend, the 2017 Design Case assumes that the immaturity of the biomass market will limit the forest and farm owner's investment in advanced equipment options for both woody and herbaceous feedstocks. Therefore, with the exception of a few proactive, early adopters, conventional forestry and farming operations will dominate the market in the regions defined by the 2017 Design Case. Table 29 below summarizes the assumptions and differences between the 2012 and 2017 logistic designs. 
Table 29. Summary of assumptions underpinning progressive design implementations ${ }^{57}$.

\begin{tabular}{|c|c|c|c|}
\hline & 2012Conventional Design & Baseline & 2017 Design Case \\
\hline Feedstock(s) & Pulpwood & & $\begin{array}{l}\text { Blended feedstock: pulpwood, wood } \\
\text { residues, switchgrass, and select } \\
\text { construction and demolition wastes (C } \\
\text { \&D) }\end{array}$ \\
\hline $\begin{array}{l}\text { Grower } \\
\text { payment }\end{array}$ & $\begin{array}{l}\text { Breakeven cost of } \\
\text { production }\end{array}$ & Pulpwood & $\begin{array}{l}\text { Calculated and modeled according to } \\
\text { specific location and resource } \\
\text { blend/formulation }\end{array}$ \\
\hline Moisture & Field dried to $40 \%$ & $\begin{array}{l}\text { Field dried to } \\
40 \%\end{array}$ & $\begin{array}{l}\text { Arrives: Pulpwood chips } 30 \% \text { wood } \\
\text { residue chips } 30 \% \text {, switchgrass } 20 \% \text {, } \\
\text { and C\& D ground } 20 \% \text {; } \\
\text { All dried to } 9 \% \text { pellets }\end{array}$ \\
\hline Ash & Debark/delimb & Debark/delimb & $\begin{array}{l}\text { Debark/delimb pulpwood } \\
\text { Trommel screen residues } \\
\text { Wash and sort C\& D waste } \\
\text { Blended ash content of }<1 \% \\
\text { Debarked pulpwood }<1 \% \text {, screened } \\
\text { wood residues } 1.4 \% \text {; washed and } \\
\text { sorted C\&D } 1.0 \%\end{array}$ \\
\hline Logistics & Uses existing systems & $\begin{array}{l}\text { Uses existing } \\
\text { systems }\end{array}$ & $\begin{array}{l}\text { Pneumatics attached to hammermill } \\
\text { High-moisture densification }\end{array}$ \\
\hline $\begin{array}{l}\text { Quality controls } \\
\text { (passive) }\end{array}$ & $\begin{array}{l}\text { Field drying to reduce } \\
\text { moisture } \\
\text { Ample available resource; } \\
\text { quality spec manually } \\
\text { selected }\end{array}$ & $\begin{array}{l}\text { Field drying to } \\
\text { meet moisture } \\
\text { spec }\end{array}$ & $\begin{array}{l}\text { Harvest/collection and storage best } \\
\text { management practices for pulpwood } \\
\text { and switchgrass } \\
\text { More rigorous field drying of } \\
\text { pulpwood and residues }\end{array}$ \\
\hline $\begin{array}{l}\text { Quality controls } \\
\text { (active) }\end{array}$ & Waste heat dryer & Rotary drying & $\begin{array}{l}\text { Multiple resource } \\
\text { blending/formulation } \\
\text { High-moisture densification } \\
\text { High-efficiency pellet drying }\end{array}$ \\
\hline $\begin{array}{l}\text { Meets quality } \\
\text { target }\end{array}$ & Yes & Yes & Yes \\
\hline $\begin{array}{l}\text { Meets cost } \\
\text { target }\end{array}$ & Yes & No & Yes \\
\hline $\begin{array}{l}\text { Accesses } \\
\text { dispersed } \\
\text { resources }\end{array}$ & No & No & Yes \\
\hline
\end{tabular}

Relative to the woody feedstocks used in the Thermochemical Design Case, the 2013 baseline and 2017 Design Case are similar in many ways for harvest and collection, but the latter has two key changes to improve quality and production of woody materials. While each system is discussed later, the key differences of the 2017 Design Case are first inclusion of woody residues 
sourced from pulpwood operations, and second in-forest drying of whole tree piles at the landing to achieve a more aggressive moisture content of 30\%. In this design debarking and delimbing are conducted to improve biomass quality. Construction and demolition wastes are considered to enter the feedstock logistics system at the preprocessing stage and are therefore not discussed here.

\subsection{Feedstock Logistics}

\subsubsection{Harvest and Collection}

Conventional wood harvest and collection relies on existing forestry technologies designed for timber and pulp and paper production. Collection systems for woody material involve cutting the feedstock with a tracked feller buncher and transporting the material to the landing with a grapple skidder immediately after felling. Felling and skidding operations increase the overall ash content of harvested whole-trees by introducing soil as it is moved in contact with the ground from one location to the next ${ }^{58}$. Thermochemical conversion processes via fast pyrolysis are highly sensitive to silica, alkali, and transition metals found in most soils, impacting process performance $^{596061}$. In both the 2013 and 2017 Design Cases active management strategies are employed during the landing preprocessing to reduce biomass ash content .Current forestry production of pine pulpwood is reported to yield roundwood with a moisture content of 45-55\% and a whole-tree ash content ranging from $1 \%$ to $3 \%{ }^{62} 6364$. The 2013 baseline assumes the moisture content of pulpwood to be $50 \%$ for whole-trees entering storage.

Woody residues are generated through typical commercial forestry operations on southern pine plantations where trees are harvested for pulpwood, chip-and-saw, and saw timber. Similar to the above described collection of pulpwood, these operations bring whole trees to the landing where they are delimbed and topped using a pull-through delimber. The roundwood is then loaded onto trucks for delivery to the mill while the residues are piled at the landing. While not collected in the 2013 baseline, the 2017 Design Case utilizes these materials as a fraction of the feedstock blend. The baseline for residue moisture content is reported at $40 \%$, while ash content has been reported to range from $2 \%$ to $4 \% 47,62,6566$. Switchgrass, which is part of the blend, harvest and collection systems use a conventional windrowing harvester and rectangular baler $(3 \times 4 \times 8-\mathrm{ft})$.

\subsubsection{Harvest and Collection Design Basis}

The 2017 Design Case incorporates a chain flail debarker during preprocessing at the landing to increase the quality of the final chipped pulpwood product. Therefore moisture and ash contents of material entering storage are not different between the 2013 and 2017 Designs. However, there is still impetus to increase the operational efficiency of roundwood collection for reducing costs ${ }^{6768}$ This can be achieved through forest management shifts to short-rotation pine plantations aimed at supplying bioenergy production, increased efficiency of harvesting machinery, and increased efficiency of grapple skidder transportation. Research conducted by Auburn University for the DOE High Tonnage Forest Biomass Project has demonstrated high capacity grapplers to increase productivity by 80 tons per productive machine hour compared to traditional systems ${ }^{58}$ Figure 31 depicts a conventional skidder and a high capacity skidder. Further development of such operational improvements will play a key role in reducing costs of clean pulp chips for thermochemical conversion. In addition, transition of forest management to 
short rotation pine plantations focused on energy use is a promising option for increasing yields; should the economics of establishment be overcome ${ }^{69}$.

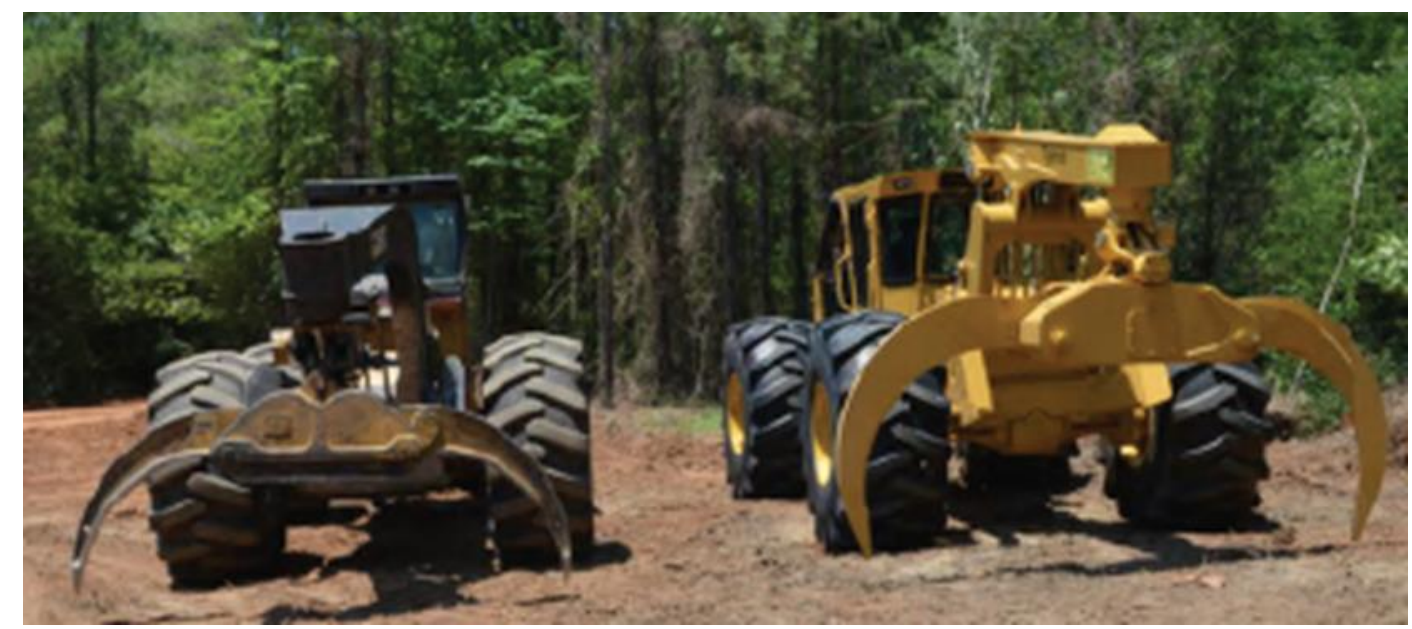

Figure 31. Conventional (left) and high-capacity grapple skidder (right) for transporting small diameter pulpwood from the forest to the landing. Photo credit: Auburn University High Tonnage Forest Biomass Project ${ }^{58}$.

Wood residues (tree tops and limbs) originate from other commercial logging operations and are located in piles at the landing, eliminating the costs for harvest and collection (e.g., felling and skidding). Similar to pulpwood, the 2017 Design Case incorporates active quality controls to reduce the ash content during preprocessing at the landing. These active controls applied after storage may contain ash contents in excess of the desired specification of $0.9 \%$ for wood residues and less for pulpwood.

Switchgrass harvest in the 2017 Design Case follows conventional practices for feed and forage in terms of the equipment used, but incorporates more rigorous passive quality controls to reduce ash content. Delayed harvest of switchgrass provides the benefits of reducing moisture and ash content, but even with the practice of delayed-harvest, it is clear that the raw feedstock will not meet the final quality specification for ash. Blending of switchgrass with a low-ash feedstock is necessary to achieve ash specification of $<1 \%$. Nevertheless, it is important that best management practices for switchgrass harvest are used to reduce soil contamination during the processes of cutting and baling while respecting the relationship between delayed harvest date and collection efficiency. Research conducted by Oklahoma State University in collaboration with INL shows that switchgrass can achieve moisture contents at or below the 2017 Design Case specification (10\% to 5\%), though climatic variance can still introduce moisture variability in delayed harvests (Figure 32). In this same research the ash content of switchgrass was found to be low even at an early harvest (5\% in August), though a decreasing trend was observed as harvest was delayed ( $4 \%$ by December). This work stands as an example of the effectiveness of proper harvesting techniques, and stresses the importance of establishing best management practices to cope with variability in weather conditions. Goals for the 2017 Design Case include reducing ash content to $4 \%$ through harvest timing and advanced harvesting techniques. 


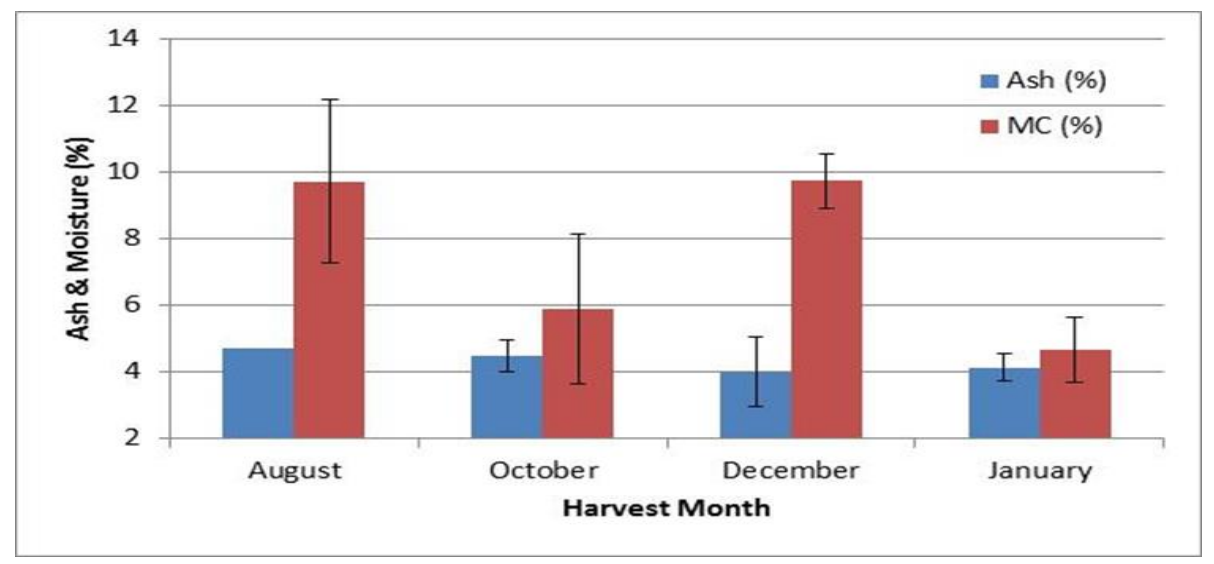

Figure 32. Ash and moisture content of switchgrass harvested in Oklahoma, 2010 by Oklahoma State University. Error bars represent one standard deviation. Ash samples for October, December, and January are three samples comprised of six individual core samples composited.

\subsubsection{Harvest and Collection Cost Estimation}

Harvest and collection costs assume a removal rate of 15 dry T./acre for pulpwood ${ }^{70}, 4$ dry T./acre for residues ${ }^{66}$, and 5-dry T./acre for switchgrass ${ }^{71}$. These assumptions are consistent with those used in the Billion Ton Update ${ }^{5}$. Cost of harvest and collection is shown in Table 30. The cost of ash at this point within the feedstock logistics system described in section 2.2 is not yet applicable to pulpwood or wood residue, as the material will undergo active quality controls during landing preprocessing. Switchgrass may be subjected to an ash dockage at this point in the process if ash contents are greater than those needed by the feedstock blending process. 
Table 30. Biomass harvest and collection cost estimates derived from INL analysis

\begin{tabular}{|lcc|}
\hline Machine & $\begin{array}{c}2013 \text { SOT } \\
\text { (2011 \$/dry T) }\end{array}$ & $\begin{array}{c}2017 \text { Target }(2011 \\
\$ / \text { dry T) }\end{array}$ \\
\hline Pulpwood & Total & Total \\
\hline Felling & & \\
Yarding & 15.00 & 15.00 \\
Totals & 7.24 & 7.24 \\
Wood Residues* & 22.24 & 22.24 \\
Felling & & \\
Yarding & 0.00 & 0.00 \\
Totals & 0.00 & 0.00 \\
\hline Switchgrass & 0.00 & 0.00 \\
Mower-conditioner & & \\
Baler & 4.80 & 4.80 \\
Bale collection/stacking & 7.30 & 7.30 \\
Totals & 3.30 & 3.30 \\
* Costed to harvest and collection of other process. & \\
\hline
\end{tabular}

\subsubsection{Storage}

\subsubsection{2013 State of Technology}

Because the 2017 Design Case utilizes a blended feedstock, switchgrass storage must be addressed. The storage of switchgrass occurs field side or at a similar on-farm unimproved storage site. As for any baled feedstock, appropriate storage sites provide adequate drainage away from the stack to prevent the accumulation of moisture around the stack, provide yearround access, and preferably allow stack to be positioned in a North-South orientation to reduce moisture accumulation on the north side of the stack ${ }^{72}$. Tarped stacks are chosen as a balance between bale protection against moisture infiltration, which leads to dry matter loss, and storage configuration costs ${ }^{73} 22$. Stacks are constructed with a self-propelled stacking bale wagon and are six bales high and covered with a high-quality hay tarp. In order to prolong tarp life, it is also important that adequate year-round maintenance be provided to periodically tighten the tarps ${ }^{74}$. Biomass storage systems in the current Design Case seek to provide a low-cost, lowmaintenance, moisture-tolerant solution that focus on maintaining moisture content $<20 \%$, minimizing dry matter loss and preserving feedstock composition. Table 31 shows the assumed changes in moisture content between the 2013 SOT and the 217 Design Case. 
Table 31. Technical targets for biomass field storage of resources in the 2017 Design Case.

\begin{tabular}{|lll|}
\hline Process & $\begin{array}{l}\text { After Field Drying } \\
\text { Moisture Content }\end{array}$ \\
\hline Pulpwood & 2013 SOT & 2017 Target \\
\hline Wood Residues & $40 \%$ & $30 \%$ \\
Switchgrass & $40 \%$ & $30 \%$ \\
\hline
\end{tabular}

\subsubsection{Storage Design Basis}

The 2017 Design Case is based on field drying for pulpwood and forest residues, both to 30\% moisture at the time the material enters landing preprocessing. Field studies on field drying of short rotation southern pine pulpwood and residues have shown final moisture contents of $30 \%$ to be achievable given adequate time ${ }^{655864}$.

Since chips are expected to enter storage at 30\% moisture in the 2017 Design Case, it is reasonable to assume that dry matter losses will be much less (nearly negligible) within the three day holding window. The concerns of unplanned storage extensions, moisture addition, or mechanical losses could increase this number, and therefore the 2017 Design Case assumes a target chip-storage dry matter loss of 5\%. Protection of chip piles with tarps could help to prevent these losses, if the additional material and labor costs are merited, and their presence does not interfere with regular loading and unloading of the piles. Storage of switchgrass is not expected to deviate from the 2013 Design Case baseline. Due to the low moisture content entering storage, the use of a tarp to protect from moisture addition through precipitation has been shown to be sufficient and cost effective when properly applied.

\subsubsection{Biomass Storage Cost Estimation}

Cost estimations for biomass storage were calculated based on literature values from recent reviews ${ }^{74}$, the storage cover vendor's information, and laboratory and field level experiments ${ }^{72}$ (Table 32).

Table 32. Field-side storage cost estimation.

\begin{tabular}{llcclll|} 
& \multicolumn{3}{c}{ SOT $(2011$ \$/dry T) } & \multicolumn{3}{c}{ Design Target $(2011$ \$/dry T) } \\
\hline \multirow{2}{*}{ Switchgrass } & Storage & Dockage & Total & Storage & Dockage & Total \\
& N/A & N/A & N/A & 5.50 & 0.00 & 5.50
\end{tabular}

\subsubsection{Preprocessing}

The 2017 Design Case that incorporates many improvements in preprocessing, including pneumatics, high-moisture densification, and formulation/blending. Figure 33 outlines the material flow given for these improvements. In the 2017 Design Case, substantial cost savings in size reduction are realized by tailoring the preprocessing stages to the individual feedstock and not applying a one size fits all approach. For example, pulpwood is debarked and delimbed and then processed through a chipper to optimize retention of usable material; wood residues are processed through a first stage grinder then separated by passing through a trommel screen; 
switchgrass is processed through a grinder while Construction and Demolition (C\&D) waste undergoes sorting and a wash step.

Separation/sorting of C\&D waste is required to remove recyclables (e.g., metal, paper, and cardboard), contaminants (e.g., plastics and concrete), and other unusable fractions to isolate only those fractions that meet the cost and quality requirements for biofuel feedstocks. In the 2017 Design Case, C\&D is sorted to supply usable material for thermochemical conversion. Prior to any preprocessing the ash content of untreated C\&D waste fractions is estimated to be about $6 \%$. The application of a wash stage further reduces the ash content down to pure wood levels of about $1 \%$.( Reference Appendix A).

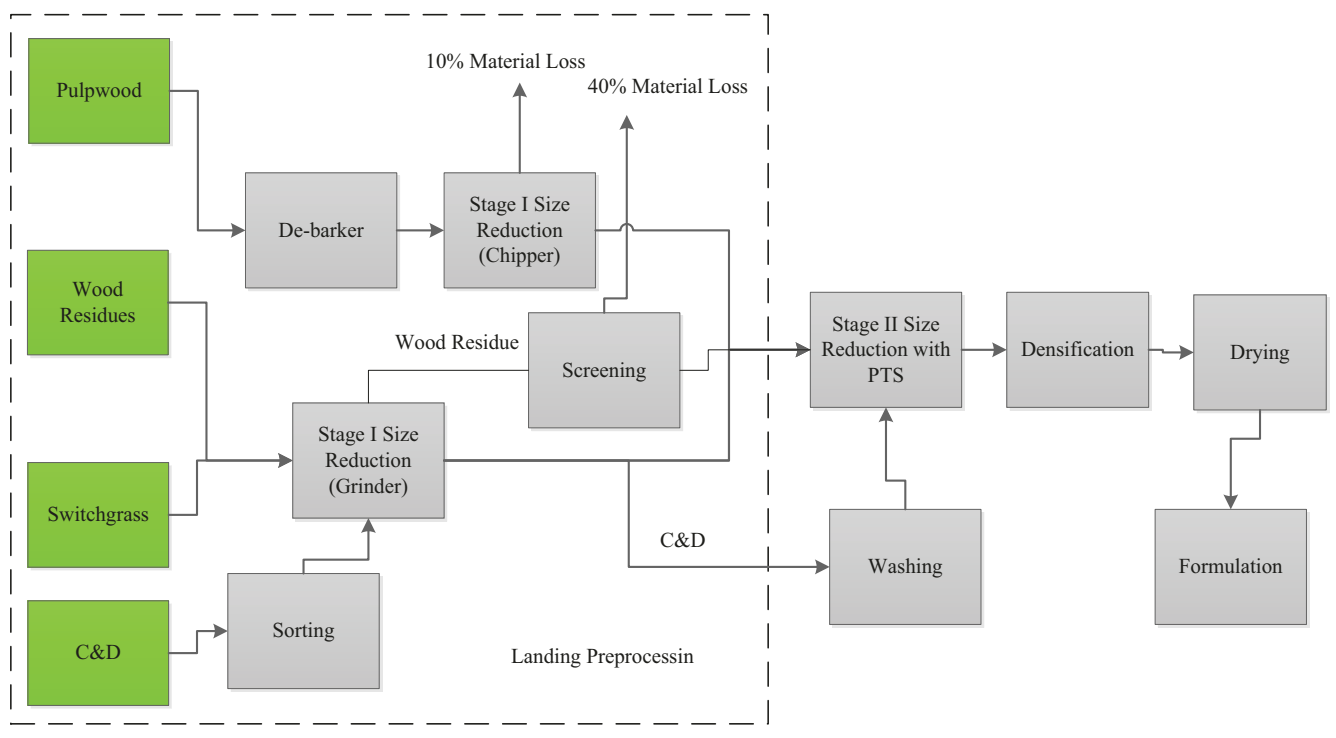

Figure 33. Material flow in the 2017 Design Case that incorporates many improvements in preprocessing, including pneumatics, high-moisture densification, and formulation/blending.

The logistics of a blended feedstock scenario are certainly more complex than a single-feedstock scenario. The 2017 Design Case assumes that preprocessing of C\&D will occur at a preprocessing depot located at the source landfill or refuse transfer station, and C\&D pellets will be shipped from the depot to the blending depot located within proximity of the biorefinery. Switchgrass that is formatted in large square bales will be delivered to the blending depot, where they will be processed into pellets. Pulpwood and wood residues will be initially processed at the landing for initial size reduction and ash mitigation then transported to a processing facility for pelletization. The pulpwood, wood residues, switchgrass, and C\&D pellets will be queued up in blending bunkers or silos and blended to specification prior to being fed into the conversion process. The pellets of the four blendstocks (i.e., pulpwood, wood residues, switchgrass, and C\&D) are then metered from the blending bunkers in the ratios required of the blended feedstock and are conveyed from the preprocessing facility/depot to the conversion facility. 


\subsubsection{State of Technology:}

For the 2017 Design Case, a geometric mean particle size of $1 / 4-$ in. is the target size specification for the thermochemical conversion process design under development by PNNL. As the target size specification is the same as biochemical conversion, size reduction system to the final particle size specification required by the end user will be the same as biochemical conversion. 2013 state of technology follows sequential two stage size reduction described in section

\subsubsection{1(Table 33).}

Table 33. Size-reduction design basis

\begin{tabular}{|lcccc|}
\hline & Equipment Used & $\begin{array}{c}\text { Screen } \\
\text { Size }\end{array}$ & $\begin{array}{c}\text { Capacity } \\
\text { Ton/hr }\end{array}$ & $\begin{array}{c}\text { Capacity } \\
\text { Ton/hr }\end{array}$ \\
\hline $\begin{array}{l}\text { First Stage Size Reduction } \\
\text { Pulpwood }\end{array}$ & Chipper & 2 in. & 17 & 17 \\
$\begin{array}{l}\text { Wood Residue } \\
\text { Second Stage Size Reduction } \\
\text { Pulpwood }\end{array}$ & Grinder & 2 in. & & \\
\hline Wood Residue & Hammer mill & $1 / 4$ in. & 5 & 6.5 \\
\hline Herget & Hammer mill & $1 / 4$ in. & 5 & 6.5 \\
\hline
\end{tabular}

\subsubsection{Size Reduction Cost Estimation}

Milling cost estimation is based on vendor-supplied information and equipment performance from typical machine performance and process demonstration unit data and is shown in Table 34.

Table 34. Size reduction cost estimates

\begin{tabular}{|ccc|}
\hline & $\begin{array}{c}2013 \text { SOT } \\
(2011 \text { \$dry T })\end{array}$ & $\begin{array}{c}2017 \text { Target } \\
(2011 \text { \$/dry T })\end{array}$ \\
\hline & Total & Total \\
\hline Pulpwood & & \\
\hline Chipper & 6.10 & 6.10 \\
\hline Debark/delimb & 6.10 & 6.10 \\
\hline Hammer Mill & 17.09 & 13.97 \\
\hline Total & $\mathbf{2 9 . 2 9}$ & $\mathbf{2 6 . 1 7}$ \\
\hline Wood Residue & & \\
\hline Grinder & 5.39 & 5.39 \\
\hline Trommel Screen & 3.32 & 3.32 \\
\hline Hammer Mill & 17.09 & 13.97 \\
\hline Total & $\mathbf{2 5 . 0 8}$ & $\mathbf{2 2 . 7 0}$ \\
\hline
\end{tabular}




\subsubsection{Drying and Densification}

Conversion of lignocellulosic biomass to hydrocarbon fuels: Fast Pyrolysis and Hydrotreating Bio-Oil Pathway use the same technology as Biological Conversion of Sugars to Hydrocarbons described in section 8.5.3.2. Therefore, state of technology and design basis for high moisture technology are the same as Biological Conversion of Sugars to Hydrocarbons described in section 8.5.3.2.

A comparison of pellet properties and energy balances for conventional and high-moisture pelletization processes is given in Table 35. The table shows 2017 Design Case targets to achieve a 40 to $50 \%$ reduction in the total pelletization and drying energy.

Table 35. Drying and densification design basis.

\begin{tabular}{|lll|}
\hline & $2013 \mathrm{SOT}$ & 2017 Target \\
\hline Infeed Moisture & $40 \%$ & $30 \%$ \\
\hline Dryer Moisture Reduction & $28 \%$ & $11 \%$ \\
\hline Densification Moisture Reduction & $3 \%$ & $10 \%$ \\
\hline Final Pellet Moisture & $9 \%$ & $9 \%$ \\
\hline Densification Energy & $75 \mathrm{kWhr} / \mathrm{dry} \mathrm{T}$ & $50 \mathrm{kWhr} / \mathrm{dry} \mathrm{T}$ \\
\hline Drying Energy & $350 \mathrm{kWhr} / \mathrm{ton}$ & $100 \mathrm{kWhr} / \mathrm{ton}$ \\
\hline Pellet Properties & & \\
\hline Unit Density & $65 \mathrm{lb} / \mathrm{ft}^{3}$ & $70 \mathrm{lb} / \mathrm{ft}^{3}$ \\
\hline Bulk Density & $35 \mathrm{lb} / \mathrm{ft}^{3}$ & $40 \mathrm{lb} / \mathrm{ft}^{3}$ \\
\hline Durability & Greater than $97.5 \%$ & $\mathrm{Greater}$ than $97.5 \%$ \\
\hline
\end{tabular}

The high-moisture densification design basis assumptions are as follows:

Our preliminary studies indicated that it is possible to produce high-quality pellets woody material; however, for our 2017 Design Case, we are assuming that the process works for other woody and herbaceous feedstocks to produce durable, high-density pellets.

Technical and cost targets are estimated with the assumption that a grain dryer will be used to dry high-moisture pellets.

Drying of pellets using energy-efficient driers like grain and belt driers is more economical compared to conventional rotary driers.

Slow drying at low temperatures of less than $60^{\circ} \mathrm{C}$ can result in more uniform moisture distribution in pellets. 


\subsubsection{Cost Estimation for High-Moisture Densification}

The cost of densification was estimated using vendor-supplied information and the capacity and energy assumptions shown in Table 36.

Table 36. Drying and densification cost estimates.

\begin{tabular}{|ccc|} 
& $\begin{array}{c}2013 \text { SOT } \\
(2011 \text { \$/dry T })\end{array}$ & $\begin{array}{c}2017 \text { Target } \\
(2011 \text { \$/dry T })\end{array}$ \\
\hline Drying & Total & Total \\
\hline Densification & 17.20 & 5.60 \\
\hline Totals & 7.70 & 4.40 \\
\hline
\end{tabular}

\subsubsection{Formulation/Blending}

To meet feedstock specifications required for various conversion pathways, formulation of specific mixtures of feedstocks will likely be required. Examples include mixing high and lowcost feedstocks to meet cost targets, mixing high and low-ash feedstocks to meet an ash target, mixing of high and low-carbohydrate feedstocks to meet a yield target, and mixing easily and poorly reactive feedstocks to meet a convertibility target. An example of blending to meet an ash and moisture specification is shown in Table 37.

Table 37. Feedstock formulation/blending of ash and moisture contents*.

\begin{tabular}{lccccc|}
$\begin{array}{l}\text { Content Delivered to } \\
\text { Biorefinery Infeed }\end{array}$ & Pulpwood & Wood Residues & Switchgrass & C\&D waste & Final Blend \\
\hline $\begin{array}{l}\text { Ash content (wt. \%) } \\
\text { Moisture content }\end{array}$ & 0.5 & 1.0 & 4.0 & 1.0 & $<\mathbf{1 \%}$ \\
(\%, wet basis) & 9 & 9 & 9 & 9 & $\mathbf{9}$ \\
HHV (lb/BTU) & 8824 & 9444 & 7557 & 8824 & $\mathbf{8 9 8 4}$ \\
\hline LHV (lb/BTU) & 7255 & 7616 & 6155 & 7255 & $\mathbf{7 3 3 7}$ \\
\hline
\end{tabular}

*Pulpwood, wood resides, and switchgrass composition data were obtained from the INL Biomass Library ${ }^{75}$. see References

1. Lanholtz, M., In Interview, Idaho National Labratory, I., Ed. Oak Ridge National Laboratory (ORNL), 2013.

2. Hess, J. R.; Kenney, K.; Ovard, L.; Searcy, E.; Wright, C. Uniform-Format Solid Feedstock Supply System: A Commodity-Scale Design to Produce an Infrastructure-Compatible Bulk Solid from Lignocellulosic Biomass; Idaho National Laboratory: 2009.

3. Searcy, E.; Hess, J. R. Uniform-Format Feedstock Supply System Design for Lignocellulosic Biomass: A Commodity-Scale Design to Produce an Infrastructure-Compatible Biocrude from Lignocellulosic Biomass; INL/EXT-09-17527; 2009.

4. Argo, A. M.; Tan, E. C. D.; Inman, D.; Langholtz, M. H.; Eaton, L. M.; Jacobson, J. J.; Wright, C. T.; Muth, D. J.; Wu, M. M.; Chiu, Y. W.; Graham, R. L., Investigation of biochemical biorefinery sizing and 
environmental sustainability impacts for conventional bale system and advanced uniform biomass logistics designs. Biofuels Bioproducts \& Biorefining-Biofpr 2013, 7 (3), 282-302.

5. DOE, U. S.; Perlack, R. D.; Stokes, B. J.; U.S. Billion-Ton Update: Biomass Supply for a Bioenergy and Bioproducts Industry; ORNL/TM-2011/224; 2011.

6. Jones, S.; Meyer, P.; Snowden-Swan, L.; Padmaperuma, A.; Tan, E.; Dutta, A.; Jacobson, J.; Cafferty, K. Process design and economics for the conversion of lignocellulosic biomass to hydrocarbon fuels fast pyrolysis and hydrotreating bio-oil pathway; PNNL-23053; Pacific Northwest National Laboratory (PNNL): 2013.

7. Carpenter, D.; Westover, T. L.; Czernik, S.; Jablonski, W., Biomass feedstocks for renewable fuel production: a review of the impacts of feedstock and pretreatment on the yield and product distribution of fast pyrolysis bio-oils and vapors. Green Chemistry 2014, 16 (2), 384-406.

8. Kenney, K. L.; Smith, W. A.; Gresham, G. L.; Westover, T. L., Understanding biomass feedstock variability; special focus issue. Advanced Feedstocks for Advanced Biofuels 2012, 4 (1), 111-127.

9. Phillips, S. D.; Aden, A.; Jechura, J.; Dayton, D.; Eggeman, T. Thermochemical ethanol via indirect gasification and mixed alcohol synthesis of lignocellulosic biomass; 2007.

10. Raveendran, K.; Ganesh, A.; Khilar, K. C., Influence of mineral matter on biomass pyrolysis characteristics. Fuel 1995, 74 (12), 1812-1822.

11. Drennen, C., Interview. Idaho National Lab, I., Ed. 2013.

12. Searcy, E. M.; Blackwelder, D. B.; Delwiche, M. E.; Ray, A. E.; Kenney, K. L. Validate the cost of feedstock at \$61.57/dry US ton for the production of ethanol via thermochemical conversion; INL/LTD11-24278; Idaho National Laboratory (INL), 2011.

13. Rothbard, M. N., Grain grades and standards - historical issue shaping the future - Hill, L.D. Journal of Economic History 1991, 51 (2), 513-514.

14. Boavida, D.; P. Abelha; I. Gulyurtlu; Valentim, B.; Sousa, M. J. L. D., A study on coal blending for reducing NOx and N2O levels during fluidized bed combustion. Clean Air 2004, 5, 175-191.

15. Jhih-Shyang, S.; Frey, H. C., Coal blending optimization under uncertainty. European Journal of Operational Research 1995, 83 (3), 452-65.

16. Reddy, D. V.; Krishna, N., Precision animal nutrition: A tool for economic and eco-friendly animal production in ruminants. Livestock Research for Rural Development 2009, 21 (3).

17. Sami, M.; Annamalai, K.; Wooldridge, M., Co-firing of coal and biomass fuel blends. Progress in Energy and Combustion Science 2001, 27 (2), 171-214.

18. Walker, J. C. F., Primary wood processing: principles and practice 2nd Edition. Springer: 2006.

19. Phanphanich, M.; Mani, S., Drying characteristics of pine forest residues. Bioresources 2010, 5 (1), 108-120.

20. Hess, J. R.; Foust, T. D.; Wright, L. L.; Sokhansanj, S.; Cushman, J. H.; Easterly, J. L.; Erbach, D. C.; Hettenhaus, J. R.; Hoskinson, R. L.; Sheehan, J. J.; Tagore, S.; Thompson, D. N.; Turhollow, A. Roadmap for agriculture biomass feedstock supply in the United States; U.S. Department of Energy, Energy Efficiency and Renewable Energy: 2003.

21. Templeton, D.; Sluiter, A.; Hayward, T.; Hames, B.; Thomas, S., Assessing corn stover composition and sources of variability via NIRS. Cellulose 2009, 16 (4), 621-639.

22. Shinners, K. J.; Boettcher, G. C.; Muck, R. E.; Weimer, P. J.; Casler, M. D., Harvest and storage of two perennial grasses as biomass feedstocks. Transactions of the ASABE 2010, 53 (2), 359-370.

23. Turn, S. Q.; Kinoshita, C. M.; Ishimura, D. M., Removal of inorganic constituents of biomass feedstocks by mechanical dewatering and leaching. Biomass Bioenerg. 1997, 12 (4), 241-252.

24. Karlen, D. L.; Muth, D. J., Landscape management for sustainable supplies of bioenergy feedstock and enhanced soil quality. Agrociencia Uruguay, Special Issue 2012.

25. Adler, P. R.; Sanderson, M. A.; Boateng, A. A.; Weimer, P. I.; Jung, H. J. G., Biomass yield and biofuel quality of switchgrass harvested in fall or spring. Agronomy Journal 2006, 98 (6), 1518-1525. 
26. Hoskinson, R. L.; Karlen, D. L.; Birrell, S. J.; Radtke, C. W.; Wilhelm, W. W., Engineering, nutrient removal, and feedstock conversion evaluations of four corn stover harvest scenarios. ScienceDirect 2007, (31), 126-136.

27. Wilhelm, W. W.; Johnson, J. M. F.; Lightle, D. T.; Karlen, D. L.; Novak, J. M.; Barbour, N. W.; Laird, D. A.; Baker, J.; Ochsner, T. E.; Halvorson, A. D.; Archer, D. W.; Arriaga, F., Vertical distribution of corn stover dry mass grown at several US locations. BioEnergy Research 2011, 4 (1), 11-21.

28. Hess, J. R.; Wright, C. T.; Kenney, K. L., Cellulosic biomass feedstocks and logistics for ethanol production. Biofuels Bioproducts \& Biorefining-Biofpr 2007, I (3), 181-190.

29. NREL Process Design and Economics for Biochemical Conversion of Lignocellulosic Biomass to Ethanol; NREL/TP-5100-47764; September, 2011.

30. Roser, D.; Mola-Yudego, B.; Sikanen, L.; Prinz, R.; Gritten, D.; Emer, B.; Vaatainen, K.; Erkkila, A., Natural drying treatments during seasonal storage of wood for bioenergy in different European locations.

Biomass Bioenerg. 2011, 35 (10), 4238-4247.

31. Kim, D.-W.; Murphy, G., Forecasting air-drying rates of small douglas-fir and hybrid poplar stacks in oregon, USA. International Journal of Forest Engineering 2013, 24 (2), 137-147.

32. He, X.; Lau, A. K.; Sokhansanj, S.; Lim, C. J.; Bi, X. T.; Melin, S., Dry matter losses in combination with gaseous emissions during the storage of forest residues. Fuel 2012, 95 (1), 662-664.

33. Jirjis, R., Effects of particle size and pile height on storage and fuel quality of comminuted Salix viminalis. Biomass Bioenerg. 2005, 28 (2), 193-201.

34. Watson, W. F.; Twaddle, A.; Hudson, J. B., Review of chain flail delimbing-debarking. International Journal of Forest Engineering 1993, 4 (2), 37-51.

35. Jirjis, R., Storage and drying of wood fuel. Biomass Bioenerg. 1995, 9 (1-5), 181-190.

36. Ferrero, F.; Malow, M.; Noll, M., Temperature and gas evolution during large scale outside storage of wood chips. European Journal of Wood and Wood Products 2011, 69 (4), 587-595.

37. Noll, M.; Jirjis, R., Microbial communities in large-scale wood piles and their effects on wood quality and the environment. Applied Microbiology and Biotechnology 2012, 95 (3), 551-563.

38. Searcy, E. M.; Blackwelder, D. B.; Delwiche, M. E.; Ray, A. E.; Kenney, K. L., Impact of Screening on Behavior during Storage and Cost of Ground Small-Diameter Pine Trees: A Case Study. Forest Products Journal 2011, 61 (7), 570-578.

39. Fuller, W. S., Chip pile storage - a review of practices to avoid deterioration and economic - losses. Tappi Journal 1985, 68 (8), 48-52.

40. Schutte-Buffalo, H. M. http://www.hammermills.com/size-reduction-product-categories-schuttebuffalo-hammermill.

41. Yancey, N. A.; Tumuluru, J. S.; Wright, C. T., Drying, Grinding and Pelletization Studies on Raw and Formulated Biomass Feedstock's for Bioenergy Applications. Journal of Biobased Materials and Bioenergy 2013, 7 (5), 549-558.

42. Tumuluru, J. S.; Sokhansanj, S.; Hess, J. R.; Wright, C. T.; Boardman, R. D., A review on biomass torrefaction process and product properties for energy applications. Industrial Biotechnology 2011, 7 (5), 384-401.

43. DOE, U. S. Bioenergy Technologies Office Peer Review 2013.

44. Davis, D.; Tao, L.; Tan, E.; Biddy, M.; Beckham, G.; Scarlata, C.; Jacobson, J.; Cafferty, K.; Ross, J.; Lukas, J.; Knorr, D.; Schoen, P. Process design and economics for the conversion of lignocellulosic biomass to hydrocarbons: dilute-acid and enzymatic deconstruction of biomass to sugars and biological conversion of sugars to hydrocarbons; Natioanl Renewable Energy Laboratory: Golden, CO, 2013. 45. CENNATEK Feasibility of improving biomass combustion through extraction of nutrients; $2011 ; \mathrm{p}$ 107.

46. Humbird, D.; Davis, R.; Tao, L.; Kinchin, C.; Hsu, D.; Aden, A.; Schoen, P.; Lukas, J.; Olthof, B.; Worley, M.; Sexton, D.; Dudgeon, D. Process design and economics for biochemical conversion of lignocellulosic biomass to ethanol; 2011. 
47. Langholtz, M.; Graham, R.; Eaton, L.; Perlack, R.; Hellwinkel, C.; Ugarte, D. G. D. 1. T., Price projections of feedstocks for biofuels and biopower in the U.S. Energy Policy 2012, 41, 484-493. 48. Bonner, I. J.; Kenney, K. L., Moisture sorption characteristics and modeling of energy sorghum (Sorghum bicolor (L.) Moench). Journal of Stored Products Research 2013, 52, 128-136.

49. Iowa-State-University, Agriculture and Natural Resources. 2013.

50. Yu, M.; Zeng, G.; Chen, Y.; Yu, H.; Huang, D.; Tang, L., Influence of Phanerochaete chrysosporium on microbial communities and lignocellulose degradation during solid-state fermentation of rice straw.

Process Biochemistry 2009, 44 (1), 17-22.

51. Selig, M.; Weiss, N.; Ji, Y. Enzymatic saccharification of lignocellulosic biomass: LAP NREL/TP510-42629 2008.

52. Dowe, N.; McMillan, J. SSF experimental protocols — lignocellulosic biomass hydrolysis and fermentation; NREL/TP-510-42630; 2001.

53. Shi, J.; Thompson, V.; Yancey, N.; Stavila, V.; Simmons, B. A.; Singh, S., Impact of mixed feedstocks and feedstock densification on ionic liquid pretreatment efficiency. Biofuels 2013, 4 (1), 63 72.

54. Searcy, E.; Flynn, P.; Ghafoori, E.; Kumar, A., The relative cost of biomass energy transport. Applied Biochemistry and Biotechnology 2007, 137, 639-652.

55. PNNL Process design and economics for the conversion of lignocellulosic biomass to hydrocarbon fuels; November 2013.

56. Service, U. S. F. Forest Resources of United States, 2012. http://www.fia.fs.fed.us/programfeatures/rpa/docs/2012\%20RPA_\%20Review\%20Draft\%20Resource\%20Tables\%2002-18-2014.pdf. 57. Idaho National Lab, I. Feedstock Supply System Design and Economics for Conversion of Lignocellulosic Biomass to Hydrocarbon Fuels - Conversion Pathway: Biological Conversion of Sugars to Hydrocarbons: The 2017 Design Case; Idaho Falls, Idaho USA: Idaho National Laboratory, 2013. 58. Taylor, S.; Bob, R.; Corley, F.; Gallagher, T.; Fasina, O.; McDonald, T.; Smidt, M., High tonnage forest biomass from southern pine. Auburn University: 2012.

59. Werther, J.; Saenger, M.; Hartge, E. U.; Ogada, T.; Siagi, Z., Combustion of agricultural residues. Progress in Energy and Combustion Science 2000, 26 (1), 1-27.

60. Lindstroem, E.; Oehman, M.; Backman, R.; Bostroem, D., Influence of sand contamination on slag formation during combustion of wood derived fuels. Energy \& Fuels 2008, 22 (4), 2216-2220.

61. Ahmad, M.; Roberts, J.; Hardiman, E.; Singh, R.; Eltis, L.; Bugg, T., Identification of DypB rhodococcus jostii RHA1 as a lignin perozidase. Biochemistry 2011, 50, 5096-5107.

62. Das, K. C.; Singh, K.; Bibens, B.; Hilten, R.; Baker, S. A.; Greene, W. D.; Peterson, J. D., Pyrolysis characteristics of forest residues obtained from different harvesting methods. Applied Engineering in Agriculture 2011, 27 (1), 107-113.

63. Bartels, D.; Sunkar, R., Drought and salt tolerance in plants. Critical Reviews in Plant Sciences 2005, 24 (1), 23-58.

64. Cutshall, J.; Greene, D.; Baker, S.; Mitchell, D., Transpirational drying effects on energy and ash content from whole-tree chipping operations in a southern pine plantation. 34th Council on Forest Engineering 2011.

65. Dukes, C. C.; Baker, S. A.; Greene, W. D., In-wood grinding and screening of forest residues for biomass feedstock applications. Biomass Bioenerg. 2013, 54, 18-26.

66. Baker, S. A.; Westbrook, M. D., Jr.; Greene, W. D., Evaluation of integrated harvesting systems in pine stands of the southern United States. Biomass Bioenerg. 2010, 34 (5), 720-727.

67. Smidt, M.; Tufts, R.; Gallagher, T., Logging efficiency and cost. In ANR-1347 - Alabama

Cooperative Extension System, 2009.

68. Hiesl, P.; Benjamin, J. G., Applicability of international harvesting equipment productivity studies in maine, USA: a literature review. Forests 2013, 4 (4), 898-921.

69. Jones, P. D.; Grado, S. C.; Demarais, S., Financial analysis of intensive pine plantation establishment. Journal of Forest Economics 2010, 16 (2), 101-112. 
70. Cunningham, K.; Barry, J.; Walkingstick, T. Managing loblolly pine stands... a to z; FSA5023; University of Arkansas Division of Agriculture, Director, Cooperative Extension Service, University of Agriculture, Cooperative Extension Service

Arkansas, December 2013, 2013.

71. McLaughlin, S. B.; Kszos, L. A., Development of switchgrass (Panicum virgatum) as a bioenergy feedstock in the United States. Biomass Bioenerg. 2005, 28 (6), 515-535.

72. Smith, W. A.; Bonner, I. J.; Kenney, K. L.; Wendt, L. M., Practical considerations of moisture in baled biomass feedstocks. Biofuels 2013, 4 (1), 95-110.

73. Cundiff, J. S.; Marsh, L. S., Harvest and storage costs for bales of switchgrass in the southeastern United States. Bioresource Technology 1996, 56 (1), 95-101.

74. Darr, M. J.; Shah, A., Biomass storage: and update on industrial solutions for baled biomass feedstocks. Biofuels 2012, 3 (3), 321-332.

75. Idaho National Laboratory, I. Biomass Analytical Library. https://inlportal.inl.gov/portal/server.pt?open=514\&objID=1350\&mode $=2$.

76. News, W. R. July 20, 2012.

77. Valkenburg, C.; Gerber, M. A.; Walton, C. W.; Jones, S. B.; Thompson, B. L.; Stevens, D. J., Municipal solid waste (MSW) to liquid fuels synthesis. Availability of feedstock and technology, 2008, 1 (PNNL-18144).

78. Shi, J.; Ebrik, M.; Yang, B.; Wyman, C., The potential of cellulosic ethanol production from municipal solid waste: a technical and economic evaluation. University of California Energy Institute: Berkeley, California, 2009.

79. Gustafson, R.; Bura, R.; Cooper, J.; McMahon, R.; Schmitt, E.; Vajzovic, A., Converting Washington lignocellulosic rich urban waste to ethanol. Ecology Publication: Washington State University 2009, 09$07-060$.

80. Yan, S.; Yao, J.; Yao, L.; Zhi, Z.; Chen, X.; Wu, J., Fed batch enzymatic saccharification of food waste improves the sugar concentration in the hydrolysates and eventually the ethanol fermentation by Saccharomyces cerevisiae H058. Brazilian Arch. Biol. Technology 2012, 55 (2), 183-192.

81. Idaho National Lab, I. Unpublished data generated at INL.

82. Cho, D. H.; Shin, S. J.; Bae, Y.; Park, C.; Kim, Y. H., Ethanol production from acid hydrolysates based on the construction and demolition wood waste using Pichia stipitis,. Bioresource Technology 2011, 102, 4439-4443.

83. Gresham, G. L.; Emerson, R.; Hoover, A.; Miller, A.; Kenney, K. L. Evolution and Development of Effective Feedstock Specifications; Milestone Completion Report, \#ID 2.1.1.1.A.ML.3; Idaho National Laboratory, 2013.

84. Jenkins, B. M., Biomass leachate treatment by reverse osmosis. Fuel Processing Technology 2003, 81, 223-246.

85. ASABE ASABE Standards: Agricultural Machinery Management Data. . http://asae.frymulti.com/standards.asp.

Appendix AAppendix for $\mathrm{C} \& \mathrm{D}$ ash data

Assumptions for the formulation design basis are as follows:

- Blended feedstocks will be selected and developed to achieve conversion yield specifications. It is currently unknown how blended feedstocks will perform in the conversion pathways. The simplest assumption is that the blended feedstocks would be the sum of performances of each individual component. There are on-going trials to test various blended feedstocks and to compare the conversion efficiencies against a single feedstocks. 
- Individual feedstocks will be pelleted at depots for shipment to biorefineries. At the biorefinery, these pelleted feedstocks will be unloaded and conveyed into individual bunkers for storage. Pellets of the different blendstocks will be metered out into the bunkers in the ratios required of the blends, crushed (using a pellet crusher), and mixed prior to insertion for the conversion process.

- Material will be metered from individual bunkers onto a conveyer and then thoroughly homogenized through this process with no segregation. Mixing of solids occurs in many industries and is often problematic when solids of varying density, shape, and size are blended. This often leads to segregation, either during the mixing or while being transported to its destination. Mixing of solids is considered a trial-and-error process due to these issues.

- The expected unit operations for formulation are shown in Table 38.

Table 38. Feedstock formulation design basis

\begin{tabular}{|c|c|c|}
\hline 2013 SOT (2011 \$/dry T) & \multicolumn{2}{|c|}{ Operating Parameters } \\
\hline & Capacity & Horsepower \\
\hline Pellet Pulverizer & 100 ton/hour & $200 \mathrm{HP}$ \\
\hline $\begin{array}{l}\text { Bulk Storage with } \\
\text { Hopper }\end{array}$ & 30 ton/hour & $30 \mathrm{HP}$ \\
\hline Conveying System & 30 ton/hour & $40 \mathrm{HP}$ \\
\hline
\end{tabular}

Research is currently ongoing at INL to examine the compatibility of various feedstocks blends, with an initial focus on the blends reactivity versus the individual feedstocks. Blends will be developed for several regions of the United States using the least-cost formulation model as a starting point and will incorporate feedstocks with varying levels of reactivity (e.g., herbaceous, woody, and MSW). Reactivity for the fermentation pathway will be investigated first, with expansion into the other DOE conversion pathways in later fiscal years including bio-oil conversion via fast pyrolysis.

While the costs for preprocessing of feedstocks (e.g., grinding, chemical preconversion, pelleting, and drying) are addressed in other parts of the 2017 Design Case, formulation itself will require a different set of preprocessing options in order to match up with the bio-oil conversion pathway. These processes include bulk storage, conveying systems and a pellet pulverizer to insure that the appropriate recipe of material enters the throat of the conversion reactor in the appropriate blends and sizing requirements.

\subsubsection{Cost Estimation for Formulation.}

Formulation cost estimation was as based on existing technology, vendor-supplied information, and equipment performance (Table 39). These costs are cursory and require more extensive research, especially in their specific application to the bioenergy industry. 
Table 39. Formulation cost estimation

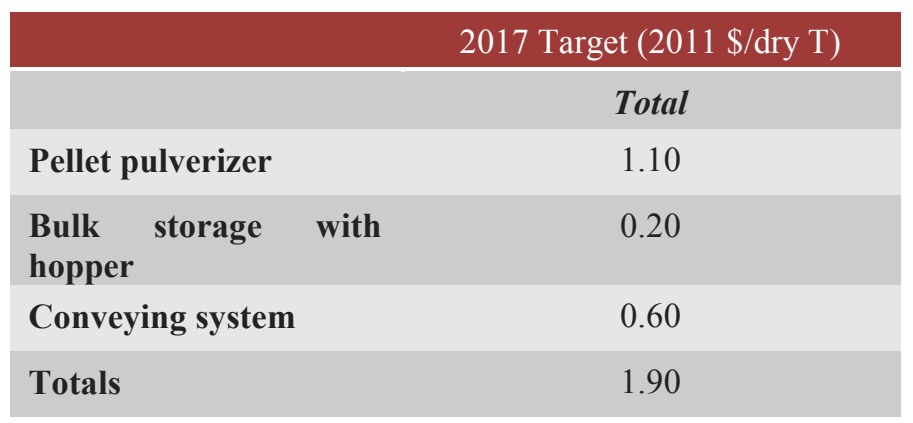

\subsubsection{Transportation and Handling Design Basis}

The 2017 Design Case includes formulation and densification that meets feedstock specifications and costs targets. Both processes of formulation and densification will improve feedstock handling operations through active controls. Given formulation and the specific quantities of individual feedstocks required, the average transportation distance will change based on feedstock type. In the 2017 Design Case, pulpwood will be trucked from a local draw radius of 50 miles while switchgrass will be trucked fewer than 15 miles. Error! Reference source not

found. in Appendix A show sufficient C\&D waste resources in the selected counties in western South Carolina; therefore, C\&D will be transported by truck from transfer stations after processing. This is not a new concept; transfer stations are already used for sorting and transporting valuable material such as cardboard and scrap metal in densified forms (e.g. baled cardboard, crushed and baled scrap metal). Switchgrass will be loaded and unloaded at each location using a loader (telehandler) capable of moving $12 \mathrm{lb} / \mathrm{ft}^{3}$ bales at $20 \%$ respective moisture content. A 53ft. trailer and 800,000-GVW limits were assumed in all trucking operations. Transportation for switchgrass will occur from a field side stack to a densification facility completely separate from the conversion facility, but within a minimal conveyor distance (typically $<50$ miles). C\&D waste transportation will occur from the waste transfer station as pellets to the preprocessing facility for storage and transfer to the biorefinery. Further transportation and handling assumptions are given as follows:

- At $20 \%$ moisture, transportation of switchgrass continues to be volume limited at densities of $12 \mathrm{lb} / \mathrm{ft}^{3}$.

- At 30\% moisture, transportation of chips continues to be volume limited.

- At $9 \%$ moisture, transportation of pellets is weight limited at $40 \mathrm{lb} / \mathrm{ft}^{3}$.

- There will be insignificant material losses throughout transportation and handling.

- Densification will increase material uniformity and flowability 


\subsubsection{Cost Estimation for Transportation and Handling}

The cost estimation for transportation and handling was based on vendor-supplied information and equipment performance from typical machines (Table 40). Rail transportation costs were based on work from Searcy using a jumbo hopper car ${ }^{54}$ adjusted for U.S. conditions.

Table 40. Transportation cost estimates.

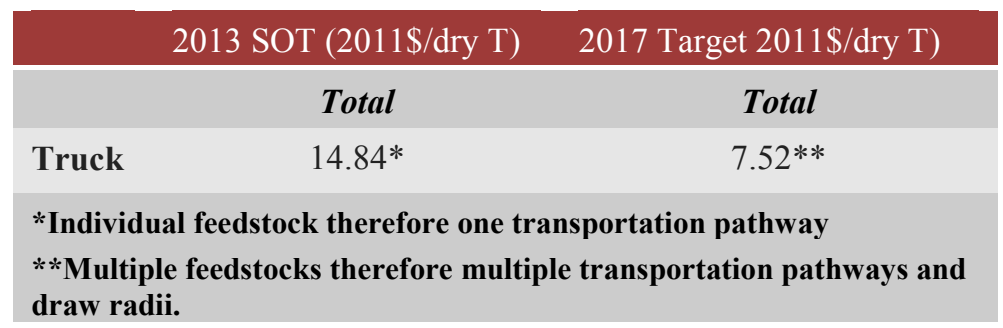

\subsection{Life Cycle Analysis}

This section uses a life-cycle assessment (LCA) to evaluate the energy input and GHG emissions for conversion of lignocellulosic Biomass to Hydrocarbon Fuels via Fast Pyrolysis and Hydrotreating Bio-Oil Pathway. LCA considered the unit processes involved in the biomass logistics. Table 41 shows the energy consumption and Table 42 shows GHG contribution for Fast Pyrolysis and Hydrotreating Bio-Oil Pathway conversion supply chain design.

Table 41. Energy Consumption for Thermochemical conversion supply chain design.

\begin{tabular}{|c|c|c|c|c|c|}
\hline Process Element & Pulpwood & $\begin{array}{l}\text { Wood } \\
\text { Residues }\end{array}$ & Switchgrass & $\begin{array}{l}\text { Construction and } \\
\text { Demolition Waste } \\
\text { (C\&D) }\end{array}$ & Blend \\
\hline Formulation Contribution & $45 \%$ & $32 \%$ & $3 \%$ & $20 \%$ & - \\
\hline $\begin{array}{l}\text { Harvest and collection } \\
(\text { MBTU/dry T) }\end{array}$ & 182.78 & - & 122.85 & - & 85.94 \\
\hline $\begin{array}{l}\text { Landing } \\
\text { Preprocessing/Sorting } \\
\text { (MBTU/dry T) }\end{array}$ & 231.52 & 110.25 & - & 410.25 & 221.51 \\
\hline $\begin{array}{l}\text { Transportation (MBTU/dry } \\
\text { T) }\end{array}$ & 136.87 & 101.38 & 99.35 & 273.73 & 151.76 \\
\hline $\begin{array}{l}\text { Preprocessing (MBTU/dry } \\
\text { T) }\end{array}$ & 408.01 & 408.01 & 285.83 & 408.01 & 404.34 \\
\hline Storage (MBTU/dry T) & 8.46 & 8.46 & 21.83 & 8.46 & 8.86 \\
\hline Handling (MBTU/dry T) & 42.69 & 42.69 & 41.90 & 42.69 & 42.67 \\
\hline $\begin{array}{l}\text { Total Energy Consumption } \\
\text { (MBTU/dry T) }\end{array}$ & 1001.33 & 670.79 & 571.76 & 1143.14 & 915.08 \\
\hline
\end{tabular}


Table 42. GHG contribution for thermochemical conversion supply chain design.

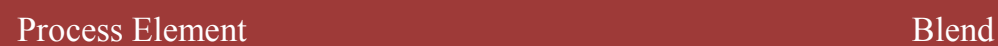

Formulation Contribution

Harvest and Collection GHGs $\left(\mathrm{Kg} \mathrm{CO}_{2} \mathrm{e} /\right.$ dry T)

8.65

Landing Preprocessing GHGs $\left(\mathrm{Kg} \mathrm{CO}_{2} \mathrm{e} /\right.$ dry T)

18.95

Transportation GHGs $\left(\mathrm{Kg} \mathrm{CO}_{2} \mathrm{e} /\right.$ dry T)

8.62

Preprocessing GHGs $\left(\mathrm{Kg} \mathrm{CO}_{2} \mathrm{e} /\right.$ dry T)

34.60

Storage GHGs $\left(\mathrm{Kg} \mathrm{CO}_{2} \mathrm{e} /\right.$ dry T)

0.89

Handling GHGs $\left(\mathrm{Kg} \mathrm{CO}_{2} \mathrm{e} /\right.$ dry T)

4.2

Total GHGs ( $\left.\mathrm{Kg} \mathrm{CO}_{2} \mathrm{e} / \operatorname{dry} \mathrm{T}\right)$

75.91 


\subsection{Dilute-Acid and Enzymatic Deconstruction of Biomass to Sugars and Catalytic Conversion of Sugars to Hydrocarbons}

This conversion path is coupled with the National Renewable Energy Laboratory's (NREL's) hydrocarbon design report, "Dilute-Acid and enzymatic deconstruction of biomass to sugars and catalytic conversion of sugars to hydrocarbons," (NREL/TP-5100-60223) that describes a single viable route from biomass to hydrocarbon fuels. Because of this coupling, the assumptions of scale and feedstock quality requirements are consistent with the design case assumptions used by NREL in their report and techno-economic assessments. In addition, this design does not consider the different requirements and nuances of other biological conversion processes or other hydrocarbon pathways. Feedstock design reports associated with alternate hydrocarbon pathways of the DOE Bioenergy Technologies Office program will follow this report.

\subsection{Summary}

Two requirements for the 2017 Design Case that were established early in this report are achieving the $\$ 80$ /dry T cost target when located outside the Midwest Corn Belt and achieving biorefinery quality specifications within the $\$ 80$ cost target. Feedstock curves were developed for the 2017 Design Case scenario located in western Kansas (Figure 34). These curves included access costs (i.e., grower payment), logistics costs, and dockage costs (e.g., ash and carbohydrate dockage). Using these curves, it was determined that a feedstock blend of $60 \%$ corn stover, $35 \%$ switchgrass, and 5\% MSW would meet the $\$ 80 /$ dry T delivered feedstock cost target, thus satisfying the cost criterion of the 2017 Design Case (Table 43). 
Table 43. Biochemical conversion feedstock design cost analysis.

\begin{tabular}{|c|c|c|c|c|c|}
\hline Cost Element & $\begin{array}{l}\text { Single-pass } \\
\text { Corn Stover }\end{array}$ & $\begin{array}{l}\text { Multi-pass } \\
\text { Corn Stover }\end{array}$ & Switchgrass & MSW & Blend \\
\hline $\begin{array}{l}\text { Formulation } \\
\text { Contribution }\end{array}$ & $35 \%$ & $25 \%$ & $35 \%$ & $5 \%$ & - \\
\hline $\begin{array}{l}\text { Grower payment/ } \\
\text { Access Cost }\end{array}$ & 27.20 & 27.20 & 29.80 & 18.00 & 27.70 \\
\hline $\begin{array}{l}\text { Harvest and } \\
\text { Collection (\$/dry T) }\end{array}$ & 10.50 & 19.20 & 15.40 & - & 13.90 \\
\hline $\begin{array}{l}\text { Transportation } \\
\text { (\$/dry T) }\end{array}$ & 8.70 & 8.30 & 7.20 & 18.00 & 8.60 \\
\hline Preprocessing (\$/dry T) & 23.40 & 23.40 & 19.70 & 19.70 & 21.90 \\
\hline Storage (\$/dry T) & 6.50 & 6.50 & 5.50 & 4.50 & 6.10 \\
\hline Handling (\$/dry T) & 1.80 & 1.80 & 1.80 & 1.80 & 1.80 \\
\hline $\begin{array}{l}\text { Total Delivered } \\
\text { Feedstock Cost }(\$ / \text { dry } \mathrm{T})\end{array}$ & 78.10 & 86.40 & 79.40 & 62.00 & $\sim 80.00$ \\
\hline \multicolumn{6}{|c|}{$\begin{array}{l}\text { Delivered Feedstock } \\
\text { Specifications* }\end{array}$} \\
\hline Ash content (wt. \%) & 3.5 & 7 & 4 & 10 & 4.9 \\
\hline $\begin{array}{l}\text { Moisture content (\%, } \\
\text { wet basis) }\end{array}$ & 9 & 9 & 9 & 9 & 9 \\
\hline $\begin{array}{l}\text { Carbohydrate content } \\
\text { (wt. \%) }\end{array}$ & 64 & 57 & 57 & 57 & 59 \\
\hline
\end{tabular}




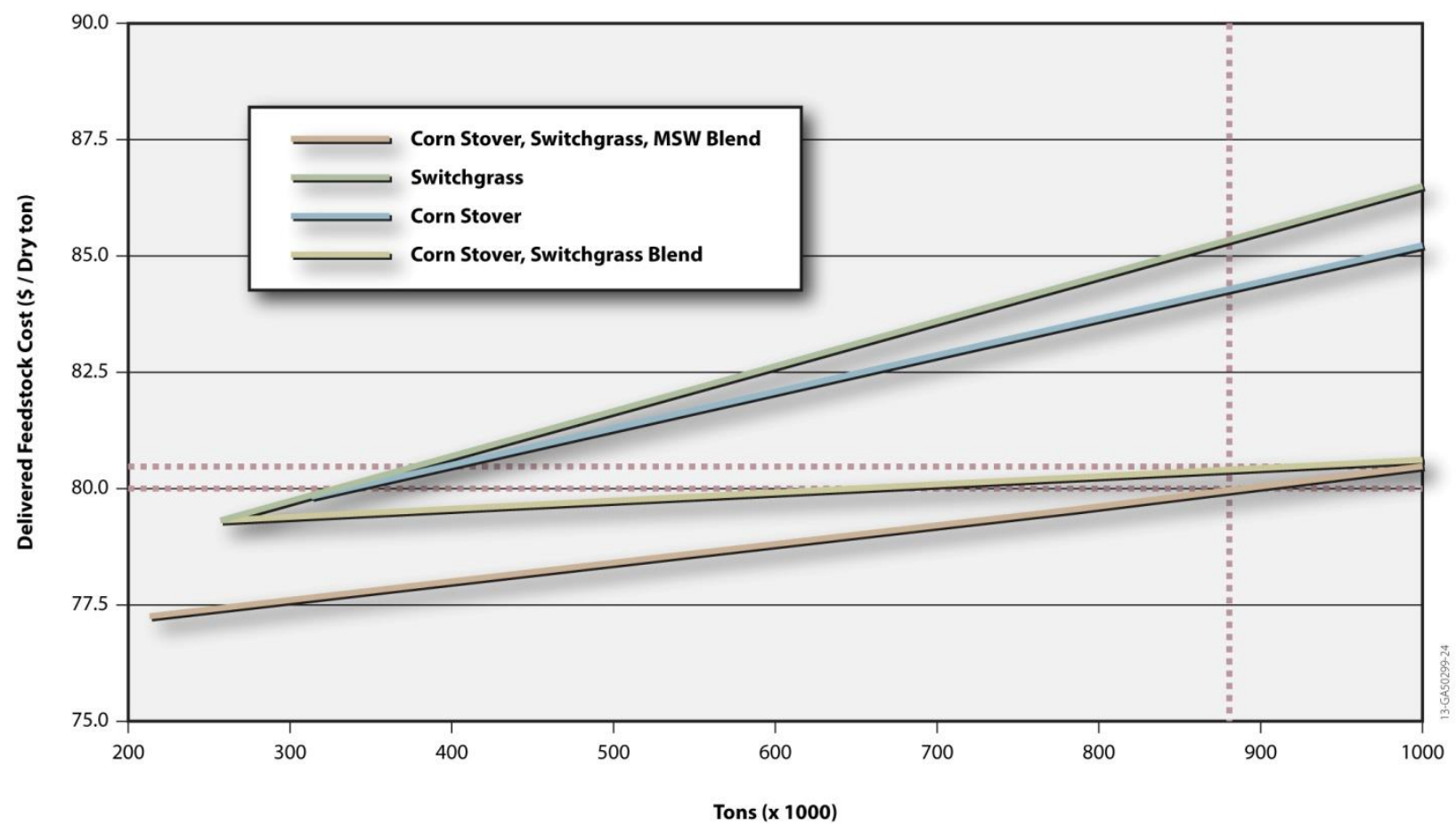

Figure 34. Comparison of individual and blended feedstock costs. A blend of $60 \%$ corn stover, $35 \%$ switchgrass, and $5 \%$ municipal solid waste is needed to hit the $\$ 80$ feedstock cost target.

Even though feedstock quality is represented in the cost curves with a dockage fee (in this case, ash dockage for multi-pass corn stover and MSW ash content in excess of the 5\% ash specification), the least-cost formulation approach does not guarantee that the lowest-cost feedstock meets spec. In fact, the $60 \%$ corn stover, $35 \%$ switchgrass, and 5\% MSW blend actually exceeded the ash specification with blended ash content of $6.1 \%$. As a result it was necessary to replace some of the higher-ash, multi-pass stover with lower-ash, single-pass corn stover in order to meet the ash specification (Table 43). The rationale for including both single and multi-pass stover is that because single pass technology is a new technology requiring additional investment by farmers, it is unlikely it will fully replace multi-pass harvest by 2017 . Sourcing 35\% single-pass and $25 \%$ multi pass corn stover assumes that about $60 \%$ of the stover will be single-pass and $40 \%$ will be multi-pass. This seems to be a reasonable assumption considering that the $60 \%$ may be harvested by a custom harvester and $40 \%$ by local farmers.

For the 2017 Design Case scenario located in western Kansas, both the cost and quality criteria could be achieved through blending. However, there may be other scenarios where reaching the $5 \%$ ash specification for biochemical conversion will require the removal of silica. Methods for accomplishing silica removal include both fine grinding followed by triboelectrostatic separation and alkali-based processes that dissolve silica ${ }^{45}$. A recent analysis for non woody feedstocks estimated a net cost of $\$ 39.93$ to $\$ 60.80$ /dry T for removal of alkali metals (up to $95 \%$ ) by leaching, followed by removal of silica (up to $75 \%$ ) by triboelectrostatic separation ${ }^{45}$. With an $\$ 80 /$ dry T feedstock cost target, these costs are too high to allow the use of chemical preconversion as an added unit operation in the current design; the existing feedstock supply 
chain operations and the grower payment leave little room for added cost. A detailed discussion of a chemical preconversion for ash removal is included in Appendix D. Therefore, for this report, we have selected feedstocks that can meet the ash specification in a blend with MSW.

The moisture and carbohydrate content of the blended feedstock also meet the specification for moisture content (i.e., less than 20\%) and carbohydrate content (i.e., at least 59\%). Because each feedstock is pelletized prior to blending, the pellets are dried to about 9 to $10 \%$ during pellet production, thereby fixing the moisture content of the blend. Similar to ash content, the carbohydrate specification is met by blending. The carbohydrate content of MSW varies depending on the particular fraction, ranging from $46 \%$ for yard waste to $64 \%$ for food waste. The MSW carbohydrate content shown in Table 43 is the average of yard waste $(46 \%)$, food waste $(64 \%)$, non-recyclable paper (55\%), and C\&D waste (61\%). Because MSW is such a small fraction of the overall blend, even food waste blends out to a carbohydrate content of $59 \%$.

\subsection{Feedstock Composition (In-feed quality specifications)}

The conversion target feedstock composition remains consistent with what was assumed in the 2011 and 2013 design cases; however, the biomass supply composition is updated relative to the original 2011 case. Previous requirements for feedstocks assumed in the 2011-era ethanol models included cost targets only for a single biorefinery. There were no quality requirements; further analysis indicated a mismatch on biomass characteristics and conversion in-feed specifications. Furthermore, this design case focuses more on supplying industry level biomass supply versus a single biorefinery. The update applied in the 2013 design case and maintained here improves on the 2011 design basis and the associated original feedstock price of $\$ 58.50 /$ dry ton $(2007 \$)$, which included a more uncertain grower payment, to a more reasonable target at an increased price of $\$ 80 /$ dry ton including grower payment (2011\$). This new price is more appropriate for a large commodity scale going beyond a "niche market" price.

As has been described in prior conversion design reports, the feedstock composition (Table 44) plays a critical role on overall process design and economics, primarily with respect to carbohydrate components (cellulose and hemicellulose), lignin, and increasingly acetate and ash, given modifications being made to the pretreatment strategy such as the use of deacetylation, as well as high sensitivity to impurity components such as ash and metals in the catalytic reactor section of this design. The blended uniform-format feedstock composition assumed here for purposes of future design case targets is shown below, with supporting details (in the context of corn stover compositional variability) described in the 2011 ethanol report ${ }^{46}$. Also consistent with prior design cases, the moisture content for the delivered feedstock is $20 \%$ or less. 
Table 44. Delivered Feedstock Composition Assumed in the Present Design ${ }^{44}$.

\begin{tabular}{|lc|}
\hline Component & $\begin{array}{c}\text { Composition } \\
\text { (dry wt \%) }\end{array}$ \\
\hline Glucan & 35.1 \\
\hline Xylan & 19.5 \\
\hline Lignin & 15.8 \\
\hline Ash & 4.9 \\
\hline Acetate & 1.8 \\
\hline Protein & 3.1 \\
Extractives & 14.7 \\
\hline Arabinan & 2.4 \\
Galactan & 1.4 \\
\hline Mannan & 0.6 \\
Sucrose & 0.8 \\
Total structural carbohydrate & 59.0 \\
Total structural carbohydrate + sucrose & 59.8 \\
\hline Moisture (bulk wt \%) & 20.0 \\
\hline
\end{tabular}

${ }^{\mathrm{a}}$ Represents acetyl groups present in the hemicellulose polymer; converted to acetic acid in pretreatment.

The current design assumes an ash target of $4.9 \%$, structural carbohydrate of $59 \%$ and a moisture of $<20 \%$.

\subsection{Feedstock Selection Cost Estimation}

Access costs are calculated from the farm gate cost based on county level supply costs as shown in Figure 35, which are derived from The Billion Ton Update ${ }^{5}$ data and are available from the Bioenergy Knowledge Discovery Framework ${ }^{25}$. Farm gate costs include nutrient replacement costs, harvest and collection costs, and an additional "profit" equal to $15 \%$ of nutrient replacement costs ${ }^{47}$. Access cost (grower payment) is calculated by subtracting the biomass harvest and collection costs from the farm gate costs. While the results are produced from ORNL's analysis using POLYSYS, the harvest and collection costs are supplied by the INL's Biomass Logistics Model. 


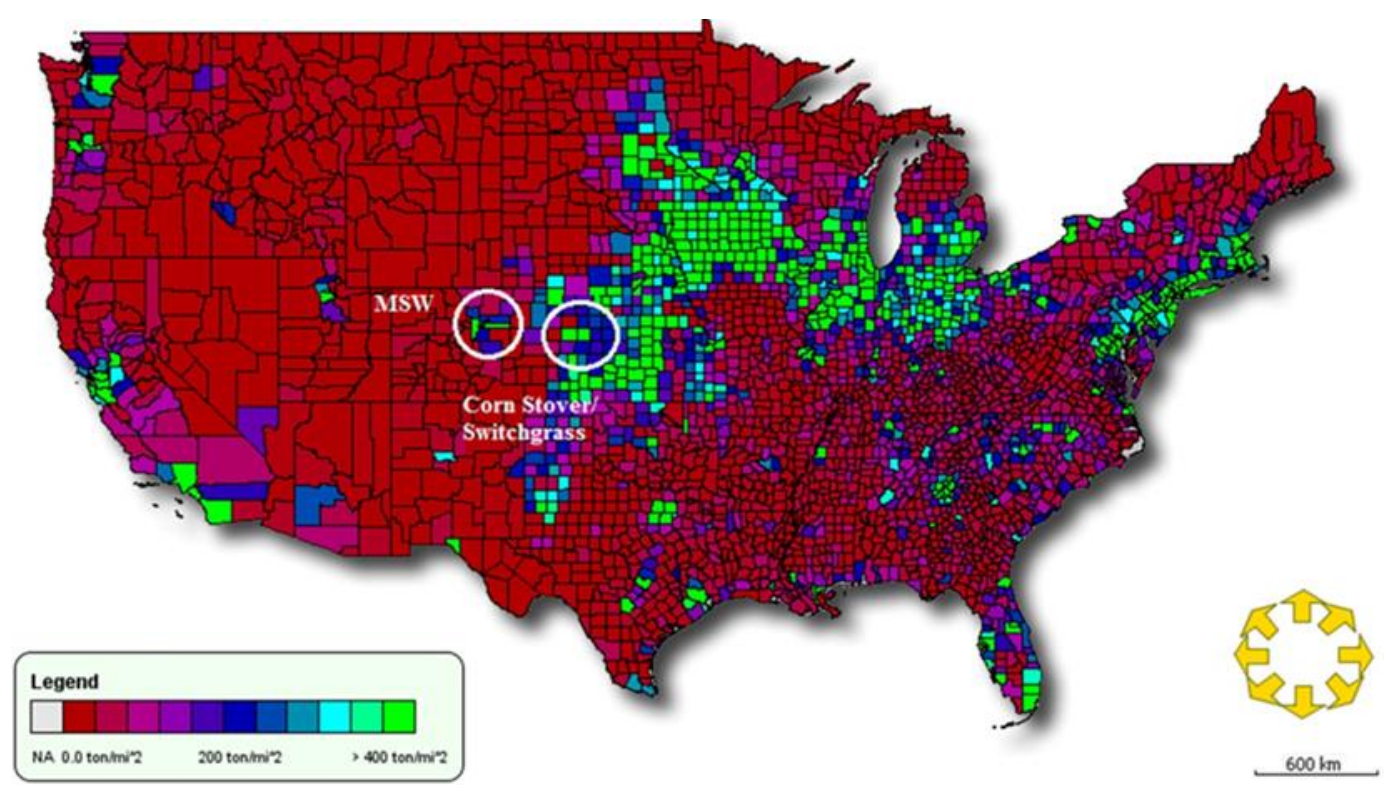

Figure 35. Resource selections for the 2017 Design Case to support biochemical conversion. Figure shows tonnages available at $\$ 40 /$ dry ton. Green represents higher amounts of tonnage available, red represents no available tonnage available at $\$ 40 /$ dry ton ${ }^{47}$.

The 2017 Design Case basis discussion presented above provided the least-cost formulation approach for reducing access costs by accessing multiple feedstocks. With this approach, reduced quantities of each feedstock that make up the total blendstock allows us to stay lower on the supply curve than if we had to supply the entire supply demand with any single feedstock. The impact of this approach is shown in Table 45. The 2013 SOT assumes a 100\% supply of corn stover and an access cost to supply 870,000 dry $\mathrm{T}$ estimated at $\$ 40 /$ ton. In comparison, the 2017 Design Case blend of 60\% corn stover, 35\% switchgrass, and 5\% MSW results in a weighted average feedstock cost of $\$ 27 /$ dry ton that is nearly $30 \%$ lower than the access cost of stover alone.

Table 45. Resource access cost estimate.

\begin{tabular}{|lclll|}
\hline & 2013 SOT & \multicolumn{3}{c|}{ 2017 Target } \\
& $\begin{array}{l}\text { Access Cost } \\
\text { (2011 \$/dry T) }\end{array}$ & Tons & $\begin{array}{l}\text { Access Cost } \\
(\mathbf{2 0 1 1} \text { \$/dry T) }\end{array}$ & Tons \\
Corn stover & 40.00 & 870,000 & 27.20 & 522,000 \\
\hline Switchgrass & NA & 0 & 29.80 & 304,500 \\
MSW & NA & 0 & 18.00 & 43,500 \\
Total & 40.00 & 870,000 & $27.70($ wt avg) & 870,000 \\
\hline
\end{tabular}




\subsection{Quality Specification and Design Assumptions}

The major assumptions of the 2017 Design Case, compared to the 2012 Conventional Design and the 2013 SOT are shown in Table 46. The implications of these assumptions on feedstock supply systems designs are discussed in following sections of the report.

Table 46. Summary of assumptions underpinning progressive design implementations.

\begin{tabular}{|c|c|c|c|}
\hline & 2012 Conventional Design & 2013 SOT & 2017 Design Case \\
\hline Feedstock(s) & Corn stover & Corn stover & $\begin{array}{l}\text { Blended feedstock: corn } \\
\text { stover, switchgrass, and } \\
\text { select municipal solid waste } \\
\text { (MSW) }\end{array}$ \\
\hline Grower payment & Minimal & $\begin{array}{l}\text { Increases based on marginal } \\
\text { cost differential }\end{array}$ & $\begin{array}{l}\text { Calculated and modeled } \\
\text { according to specific } \\
\text { location and resource } \\
\text { blend/formulation }\end{array}$ \\
\hline Moisture & Field dried to $12 \%$ & $\begin{array}{l}\text { Arrives at } 30 \% \\
\text { Dried to } 20 \%\end{array}$ & $\begin{array}{l}\text { Arrives: corn stover } 30 \% \text {, } \\
\text { switchgrass } 20 \% \text {, and } \\
\text { MSW } 20 \% \text {; } \\
\text { All dried to } 7 \%\end{array}$ \\
\hline Ash & No ash management assumed & $\begin{array}{l}11 \% \text {, dockage accessed for } \\
\text { ash content Greater than } 5 \% \\
\text { spec }\end{array}$ & $\begin{array}{l}\text { Blended ash content of } 4.9 \% \\
\text { Corn stover: multi-pass } 7 \% \text {; } \\
\text { single-pass } 3.5 \% \\
\text { Switchgrass: } 4 \% \\
\text { MSW: } 10 \%\end{array}$ \\
\hline Logistics & Uses existing systems & Uses existing systems & $\begin{array}{l}\text { Fractional milling } \\
\text { High-moisture densification } \\
\text { Rail transportation for MSW }\end{array}$ \\
\hline $\begin{array}{l}\text { Quality controls } \\
\text { (passive) }\end{array}$ & $\begin{array}{l}\text { Field drying to meet moisture } \\
\text { spec } \\
\text { Ample available resource; } \\
\text { quality spec manually } \\
\text { selected }\end{array}$ & $\begin{array}{l}\text { Dockage fee assessed to } \\
\text { supplier for below-quality } \\
\text { material }\end{array}$ & $\begin{array}{l}\text { Multi versus single-pass } \\
\text { harvest/ collection } \\
\text { Harvest/collection and } \\
\text { storage best management } \\
\text { practices }\end{array}$ \\
\hline $\begin{array}{l}\text { Quality controls } \\
\text { (active) }\end{array}$ & None assumed & Rotary drying & $\begin{array}{l}\text { Multiple resource } \\
\text { blending/formulation } \\
\text { High-moisture densification } \\
\text { High-efficiency pellet } \\
\text { drying }\end{array}$ \\
\hline Meets quality target & No & Yes & Yes \\
\hline Meets cost target & Yes & No & Yes \\
\hline $\begin{array}{l}\text { Accesses dispersed } \\
\text { resources }\end{array}$ & No & No & Yes \\
\hline
\end{tabular}




\subsection{Feedstock Logistics}

\subsubsection{Harvest and Collection}

\subsubsection{Overview}

The 2012 Conventional Design focused on conventional multi-pass harvest methods (i.e., the mowing and/or windrowing operations are separate from the baling operation). Single-pass harvesting systems (such as those developed through the DOE-funded, high-tonnage, logistics projects) offer efficiency and quality improvements over conventional, multi-pass systems. The 2017 Design Case assumes that the immaturity of the biomass market will limit the farmer investment in advanced equipment options. Therefore, with the exception of a few proactive, early adopters, conventional, multi-pass systems will dominate the marketplace in the regions defined by the 2017 Design Case.

In the 2017 Design Case, corn stover is harvested using a flail shredder, which is commonly referred to as a stalk chopper. The ability of a stalk chopper to minimize soil pickup and contamination compared to alternate methods drives this decision ${ }^{48}$. Corn stover harvest occurs within a 6-week window that coincides with grain harvest. In this operation, stalk chopping and baling (i.e., $3 \times 4 \times 8$-ft large, square bales) immediately follow grain harvest. The 2017 Design Case assumes a stalk chopper collection efficiency (i.e., removal rate) of about $40 \%$, with a corn stover moisture content up to $30 \%$ (wet basis). It also assumes that field drying to a preferred moisture content (i.e., less than $20 \%$ ) for long-term storage may not always be possible, resulting in corn stover bales with up to $30 \%$ moisture content that must be appropriately managed in storage. While drying in storage may occur, high-moisture biomass undergoes dry matter loss early in storage, resulting in both feedstock loss and compositional changes ${ }^{22}$.

Switchgrass harvest in the 2017 Design Case also follows conventional practices. Following plant senescence in the fall, when plant nutrients retreat into the root system and the plant naturally dries down, switchgrass is cut and windrowed using a self-propelled mowerconditioner; then it is subsequently baled using a large-square (i.e., $3 \times 4 \times 8$-ft) baler. A collection efficiency of $90 \%$ and bale moisture content of less than $20 \%$ is assumed in cost estimation for switchgrass harvest and collection.

\subsubsection{Harvest and Collection Design Basis}

The 2017 Design Case uses traditional harvest and collection equipment (i.e., both multi-pass and single-pass methods); however, it depends heavily on advancing operational strategy. Maintaining the design specifications shown in Table 47 for ash and moisture content requires a balance between harvested biomass quality, storage behavior, and final delivered feedstock specification. The blending strategy used by the 2017 Design Case merges the benefits of multipass and single-pass systems to enforce ash avoidance during harvest and reduce ash enrichment throughout storage. To meet the delivered feedstock specifications, the harvest and collection task relies on the adjustment of pre-storage goals for ash content, moisture content, and biomass yield. 
Table 47. Technical targets for harvest and collection of herbaceous resources in the 2017 Design Case.

\begin{tabular}{lcccccc|}
\hline & \multicolumn{2}{c}{ Ash Content } & \multicolumn{2}{c}{ Baled Moisture } & \multicolumn{2}{c}{ Bulk Density } \\
\hline Multi-pass & 2013 SOT & 2017 Target & 2013 SOT & 2017 Target & 2013 SOT & 2017 Target \\
Single-pass & $10 \%$ & $7 \%$ & $30 \%$ & $30 \%$ & $12 \mathrm{lb} / \mathrm{ft}^{3}$ & $12 \mathrm{lb} / \mathrm{ft}^{3}$ \\
Switchgrass & $6 \%$ & $3.5 \%$ & $30 \%$ & $30 \%$ & $12 \mathrm{lb} / \mathrm{ft}^{3}$ & $12 \mathrm{lb} / \mathrm{ft}^{3}$ \\
\hline
\end{tabular}

The 2017 Design Case focuses on improvements to and optimization of conventional equipment. Single-pass and advanced, multi-pass harvesting systems (i.e., specialized combine operation or windrowing equipment) that provide the lowest ash content feedstock will emerge first in the highly productive regions, where the economics of a single-feedstock market allow farmers to spread their investment across more acres and tons of biomass. In less productive areas, conventional multi-use systems will be operated with greater focus on reducing feedstock moisture content and improving storage stability to avoid ash enrichment throughout storage. Idaho National Laboratory (INL) research shows that stover ash content from conventional multi-pass collection equipment can approach the 2017 goal of $7.5 \%$ ash. However, additional improvements are required to minimize the uncertainty of soil entrainment while maximizing biomass yield and sustainability.

\subsubsection{Harvest and Collection Cost Estimation}

Harvest and collection costs assume a sustainable removal rate of 1.2 dry T/acre for corn stover (both single and multi-pass) and 5-dry T/acre for switchgrass. These assumptions are consistent with those used in the Billion Ton Update ${ }^{5}$. Cost reductions from the 2013 SOT to the 2017 Design Case are largely attributed to the transition from multi-pass corn stover harvest in the 2013 SOT to single-pass harvest in the 2017 Design Case (Table 48). These cost reductions are attributed to both a reduction in ash, and an improvement in the overall efficiency of the harvest operations that result from single-pass harvesting. The cost of ash is estimated from the ash dockage $\$ 2.25 /$ dry T per percent ash presented in Section 2.2.2. Ash dockage contributed $\$ 14 /$ dry T to the 2013 SOT costs. Cost of each machine consists of ownership cost and operations cost. Details are listed in Appendix E. However, the 2017 Design Case assumes that, with improvements to multi-pass harvest systems and through increased adoption of single pass harvesting equipment, the blended feedstock ash content is within specifications, thereby eliminating an ash dockage. Additional cost savings are realized through improved bale densities that result from anticipated improvements in the high-density baling technology. 
Table 48. Biomass harvest and collection cost estimates.

\begin{tabular}{|c|c|c|}
\hline \multirow[t]{2}{*}{ Machine } & $\begin{array}{c}2013 \text { SOT } \\
(2011 \text { \$/dry T) }\end{array}$ & $\begin{array}{c}2017 \text { Target (2011 } \\
\text { \$/dry T) }\end{array}$ \\
\hline & Total & Total \\
\hline \multicolumn{3}{|l|}{ Multi-pass corn stover } \\
\hline Combine* & 0.00 & 0.00 \\
\hline Shredder & 5.30 & 5.30 \\
\hline Baler & 10.60 & 10.60 \\
\hline Bale collection/stacking & 3.30 & 3.30 \\
\hline Ash dockage & 14.00 & 0.00 \\
\hline Totals & 33.20 & 19.20 \\
\hline \multicolumn{3}{|l|}{ Single-pass corn stover } \\
\hline Combine* & 0.00 & \\
\hline Baler & 7.20 & 7.20 \\
\hline Bale collection/stacking & 3.30 & 3.30 \\
\hline Totals & 10.50 & 10.50 \\
\hline \multicolumn{3}{|l|}{ Switchgrass } \\
\hline Mower-conditioner & 4.80 & 4.80 \\
\hline Baler & 7.30 & 7.30 \\
\hline Bale collection/stacking & 3.30 & 3.30 \\
\hline Totals & 15.40 & 15.40 \\
\hline
\end{tabular}

\subsubsection{Storage}

The 2017 Design Case assumes that storage of corn stover and switchgrass will occur field side or at a similar unimproved storage site. Biomass storage systems in the 2017 Design Case seek to provide a low-cost, low-maintenance, moisture-tolerant solution that focuses on the predictability of dry matter losses and compositional changes to inform an active inventory management approach to large-scale, long-term storage.

\subsubsection{Biomass Storage Design Base}

The 2017 Design Case is based on material entering storage with $30 \%$ moisture. While it is recognized that this condition is not the norm for many areas and that storage performance will vary accordingly, use of this approach ensures the supply system will be capable of dealing with unstable, non-ideal feedstock. According to INL data shown in Figure 36, we assume that 30\% moisture corn stover accumulates, at-worst, 12\% dry matter loss after about 150 days in storage (adjusted for time scale) if additional moisture is not inserted. This upper limit for dry matter loss was assumed for the entire year's lot of feedstock. 

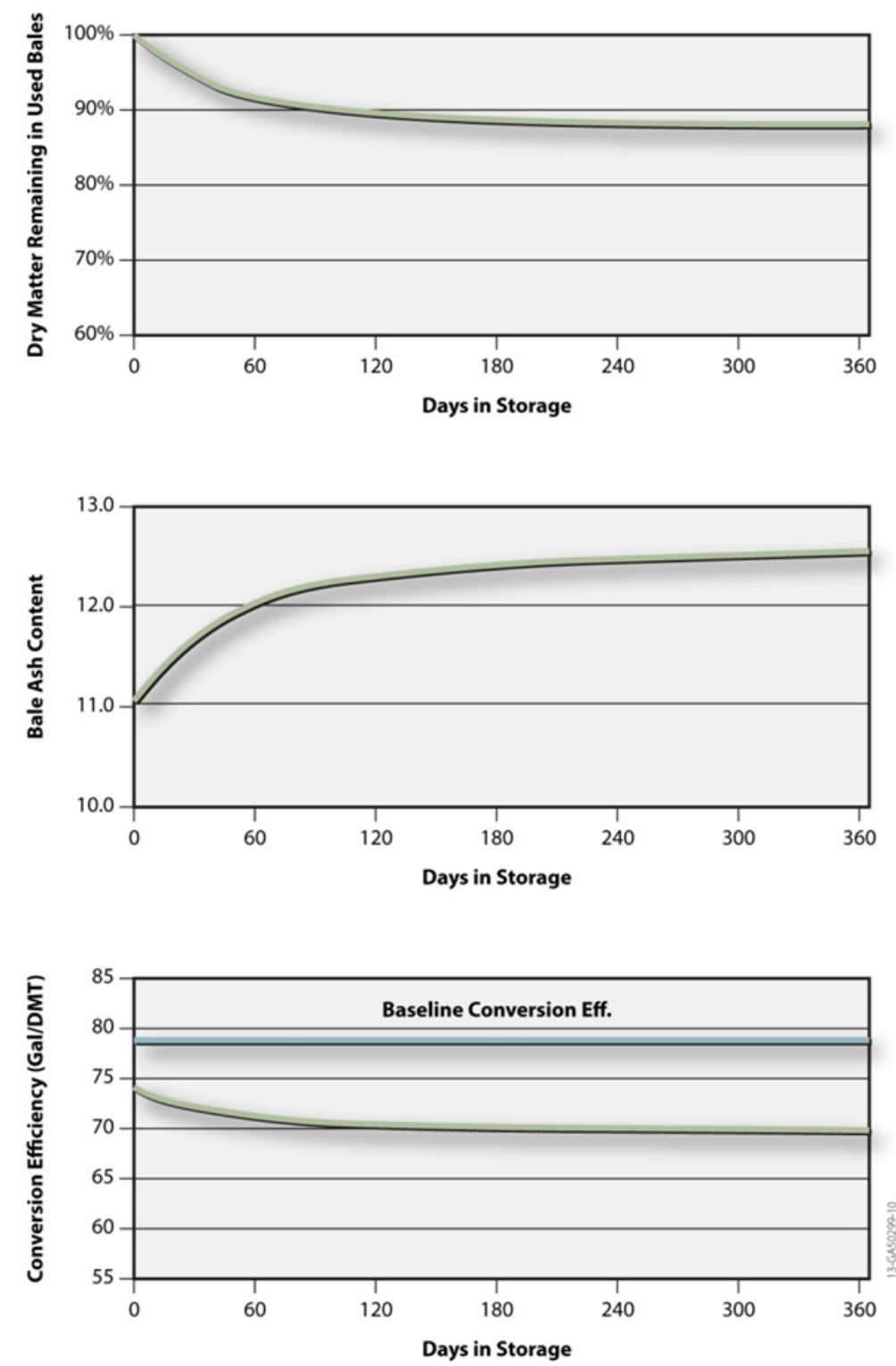

Figure 36. The impact of dry matter loss on bale ash content and final conversion efficiency (based on a $30 \%$ initial moisture and $12 \%$ ash).

As discussed in terms of the 2013 SOT, the passive loss of moisture during storage using conventional practice cannot be depended on as means to safely store wet feedstock. Therefore, storage practices developed by 2017 must be capable of limiting dry matter loss and its associated impact on convertibility, even when moisture contents entering storage are not favorable. To this end, the reduction of dry matter loss will be achieved through actively controlled improvements to storage in a way that moisture loss can be reliably achieved and/or oxygen availability can be limited in baled storage; both of which effectively limit microbial growth. Laboratory testing at INL has demonstrated that the availability of oxygen (while 
maintaining an aerobic storage environment) can effectively reduce the rates of dry matter loss in storage (Figure 37). These high-moisture corn stover samples (i.e., 50\% wet basis) demonstrate how oxygen limitation can extend the shelf life in aerobic storage. Ongoing research will determine how practical measures, such as increasing bale density, high-density stacking configurations, and tarping, can be used to limit oxygen availability and improve storage stability in high-moisture, baled, and bulk stored feedstocks.

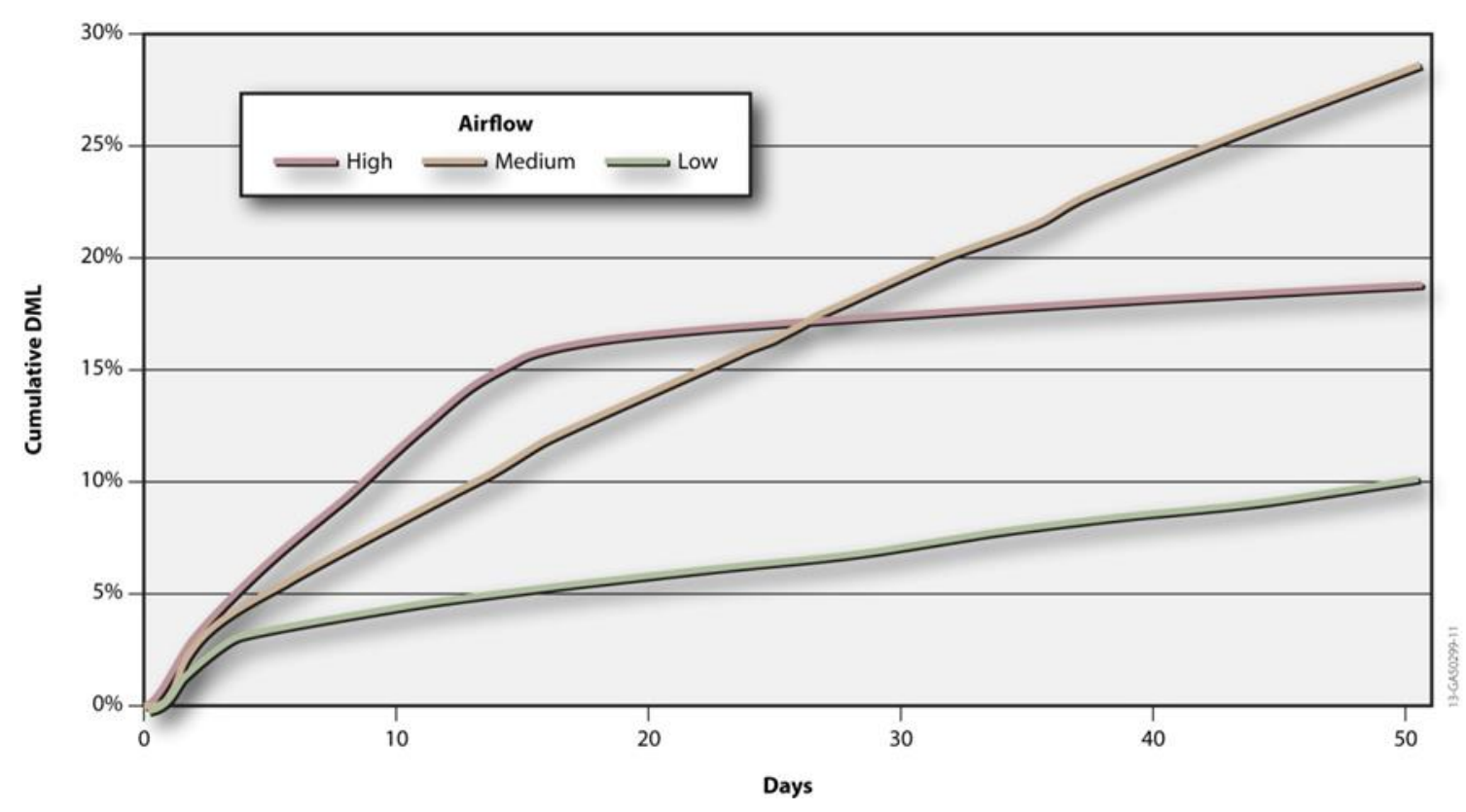

Figure 37. Dry matter loss of corn stover in the simulated storage conditions, with three air flows simulating three different oxygen availabilities.

The 2017 Design Case shifts the traditional focus of storage management away from a singular goal of minimizing dry matter loss to a more informed focus on the final material's convertibility. This approach allows the conversion yield, reasonably derived from stored biomass, to be assessed in addition to the mass loss incurred. The 2017 Design Case assumes that structural carbohydrates consumed during storage leave the remaining dry matter less convertible than the starting material. As an example of this effect, a hypothetical analysis of a storage scenario using the 2013 Base Case feedstock (30\% moisture and $12 \%$ ash) was cast in terms of the existing biochemical ethanol conversion pathway ${ }^{46}$. Regardless of final product class (e.g., ethanol versus bio-based hydrocarbon fuels), it is assumed the decreased conversion performance due to degradation in storage will have comparable impacts on the feedstock supply system, with actual impacts dependent on product-specific conversion specifications. For the purpose of this analysis, calculation in terms of ethanol presents the opportunity for a direct comparison of feedstock performance and should not be inferred as yield goals for 2017 . The analysis shows a conversion efficiency drop to $70 \mathrm{gal} / \mathrm{dry} \mathrm{T}$, which is an $11 \%$ reduction compared to the baseline of $79 \mathrm{gal} /$ dry $\mathrm{T}$ (Figure 36). The analysis assumes that dry matter losses are confined to the nonash biomass fraction, dry matter loss occurs proportionally across all non-ash components, and for each $1 \%$ dry matter lost, there is a $0.25 \%$ decrease in conversion efficiency, which is defined 
as a reduction in final product yield. As a result, when dry matter loss is accumulated over time in storage (Figure 36, top), several important behaviors and interactions are occurring, primarily the relative ash content of the material is becoming enriched (Figure 36, middle), causing the carbohydrate fraction of the biomass to respectively diminish (deviance from carbohydrate quantity spec), and the conversion performance of the remaining biomass is being reduced (deviance from the carbohydrate quality spec; Figure 36, bottom). These actions impact replacement costs, operational costs, and disposal costs for the refinery because more biomass must to be procured (replacement costs), more biomass must be handled and treated throughout the conversion process (operational costs), and more waste is being generated (disposal costs). In the 2013 Base Case, where feedstock price is $\$ 121.60 /$ dry $T$, these costs result in a total feedstock dockage of $\$ 18.93 /$ dry $T$, comprised of $\$ 12.48$ /dry T from feedstock replacement, $\$ 4.16 /$ dry $T$ from operational costs, and $\$ 2.28 /$ dry $T$ from disposal costs. Of these costs, dry matter loss is responsible for $\$ 6.10 /$ dry $\mathrm{T}$.

The technical targets for 2017 reduce this cost through decreases in dry matter loss (i.e., structural sugar quantity and quality preservation) and the ash entering storage. When the above simulation is applied to the 2017 Design Case specifications (i.e., 30\% moisture, $4.9 \%$ ash, annual dry matter loss of $7 \%$, and a $\$ 81.60 /$ dry $\mathrm{T}$ feedstock price), the dry matter loss results in a total convertibility dockage of $\$ 3 /$ dry $\mathrm{T}$ (Table 49 ). These reductions in storage-related losses will be achieved by 2017 through the minimization of microbial activity in storage; principally, through controlled limitation of moisture content and/or oxygen in stored herbaceous feedstock.

Table 49. Biomass storage design basis

\begin{tabular}{|c|c|c|c|c|}
\hline & SOT & Target & SOT & Targets \\
\hline & \multicolumn{2}{|c|}{ Dry Matter Loss } & \multicolumn{2}{|c|}{ Convertibility $^{a}$} \\
\hline Corn Stover & $12 \%$ & $8 \%$ & $59 \mathrm{gal} / \mathrm{dry} \mathrm{T}$ & 79 gal/dry T \\
\hline Switchgrass & $12 \%$ & $6 \%$ & $59 \mathrm{gal} / \mathrm{dry} \mathrm{T}$ & $79 \mathrm{gal} / \mathrm{dry} \mathrm{T}$ \\
\hline
\end{tabular}

\section{Biomass Storage Cost Estimation}

Cost estimations for biomass storage were calculated based on the storage cover vendor's information and laboratory and field level experiments (Table 50).

Table 50. Field-side storage cost estimation.

\begin{tabular}{cccccc|}
\hline \multicolumn{2}{|c}{ SOT $(2011$ \$ dry T) } & \multicolumn{4}{c|}{ Design Target $(2011$ \$/dry T) } \\
\hline Storage & Dockage & Total & Storage & Dockage & Total \\
$\mathbf{4 . 3 0}$ & 6.10 & 10.40 & 3.50 & 3.00 & 6.50 \\
\hline
\end{tabular}




\subsubsection{Preprocessing}

Biomass preprocessing operations of the 2017 Design Case differ substantially from the current state of technology, including improvements to size reduction (milling) and drying processes and the inclusion of new preprocessing operations (e.g., chemical preconversion and formulation) for ash reduction and feedstock blending. Biomass preprocessing begins with a coarse (i.e., Stage 1) size reduction to break the bale and facilitate the subsequent separations process. The next step is to separate the fractional material into two streams, one stream needing further grinding and the other stream that is at final size. The objective of biomass separations is to reduce the quantity of material that requires further preprocessing, differentiating among anatomical or size fractions based on size, material properties (e.g., moisture and density), and/or composition. In the 2017 Design Case, substantial cost savings in size reduction are realized by separating the fraction of the biomass that meets the particle size specification as it exits the Stage 1 size-reduction process, passing only the remaining over-sized materials on to the Stage 2 size-reduction process.

Separation/sorting of MSW is required to remove recyclables (e.g., metal, paper, and cardboard), contaminants (e.g., plastics and concrete), and other unusable fractions to isolate only those fractions that meet the cost and quality requirements for biofuel feedstocks. In the 2017 Design Case, MSW is sorted to supply only yard and construction/demolition waste, which consists mainly of wood waste (e.g., tree trimmings and lumber), as a feedstock to be blended with corn stover and switchgrass. The ash content of these select MSW fractions is estimated to be about $10 \%$. Chemical preconversion will be necessary for additional ash reduction (see Appendix A). Following final milling of over-sized materials to the particle-size specification (i.e., 1/4-in. minus), feedstocks are pelletized.

The 2017 Design Case incorporates many improvements in preprocessing, including fractional milling, chemical preconversion, high-moisture densification, and formulation/blending. Figure 38 demonstrates the material flow given for these improvements.

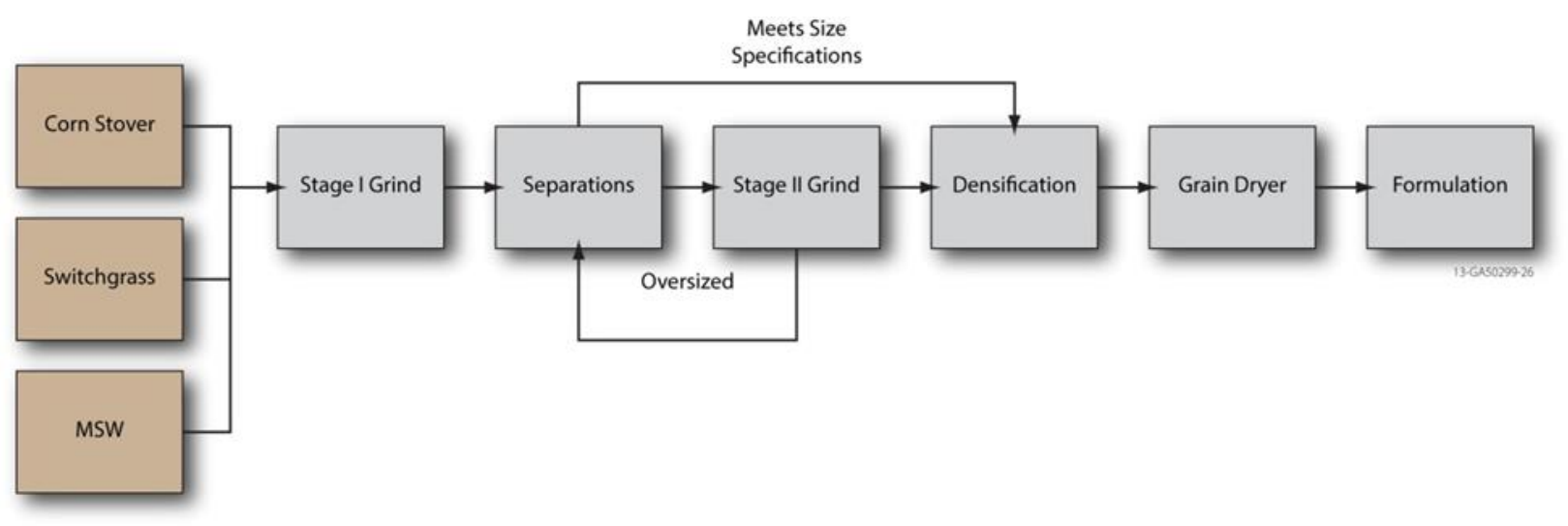

Figure 38. Material flow in the 2017 Design Case that incorporates many improvements in preprocessing, including fractional milling, chemical preconversion, high-moisture densification, and formulation/blending. 
The logistics of a blended feedstock scenario are certainly more complex than a single-feedstock scenario. The 2017 Design Case assumes that preprocessing of MSW will occur at a preprocessing depot located at the source landfill or refuse transfer station, and MSW pellets will be shipped from the depot to the blending depot located within proximity of the biorefinery. Corn stover and switchgrass that is formatted in large square bales will be delivered to the blending depot, where they will be processed into pellets. Corn stover, switchgrass, and MSW pellets will be queued up in blending bunkers or silos. The pellets of the three blendstocks (i.e., corn stover, switchgrass, and MSW) are then metered from the blending bunkers in the ratios required of the blended feedstock and are conveyed from the preprocessing facility/depot to the conversion facility.

\subsubsection{Size Reduction}

For the 2017 Design Case, a geometric mean particle size of 1/4- in. is the target size specification optimal for densification. Particle size after grinding is dictated by a number of factors, including biomass physical and material properties, process variable of the comminution system, shear and impact forces imparted by the comminution system, and the size opening of the screen used to retain material in the system until the material is sufficiently processed to pass through the screen.

Hammer mills generally are considered the current state of technology for biomass comminution due to their high throughputs and versatility in processing a wide range of materials. As a general rule of thumb, the geometric mean particle size achieved by hammer milling typically is an order of magnitude smaller than the screen size opening.

The fractional milling design basis is summarized in Table 51. Preprocessing starts with an initial (Stage 1) coarse size reduction using a 400-hp horizontal grinder configured with a 6-in. screen. Upon exiting the first-stage grinder, the coarse-ground material passes through a separator that is configured with a $1 / 4$-in. screen. The fraction that meets the size specification will pass through the screen and move onto densification, while the fraction that is retained on the screen will be conveyed into the second-stage size-reduction process for final milling to the particle size specification. The fractional milling process will reduce the total effective energy consumption for biomass size reduction by about 60 and $70 \%$ for dry $(15 \%)$ and wet $(30 \%)$ biomass, respectively. Note that this calculation is based on the effective energy consumption for second-stage comminution (see footnote to Table 51). 
Table 51. Size-reduction design basis

\begin{tabular}{|c|c|c|c|c|}
\hline & 2013 SC & & $2017 \mathrm{Ta}$ & \\
\hline & Stage 1 & Stage 2 & Stage 1 & Stage 2 \\
\hline Screen size & $2 \mathrm{in.}$ & $1 \mathrm{in.}$ & 6 in. & $1 \mathrm{in.}$ \\
\hline $\begin{array}{l}\text { Comminution energy at } 15 \% \text { moisture } \\
(\mathrm{kWh} / \text { dry } \mathrm{T})\end{array}$ & 39 & 21 & 10 & $21 *$ \\
\hline $\begin{array}{l}\text { Comminution energy at } \quad 30 \% \\
\text { moisture(kWhr/dry T) }\end{array}$ & 40 & 60 & 15 & $21 *$ \\
\hline $\begin{array}{l}\text { Separations at } 15 \% \text { moisture (percent } \\
\text { passing } 1 / 4 \text {-in. screen) }\end{array}$ & 100 & 100 & 100 & 55 \\
\hline $\begin{array}{l}\text { Separations at } 15 \% \text { moisture (percent } \\
\text { passing } 1 / 4 \text {-in. screen) }\end{array}$ & 100 & 100 & 100 & 55 \\
\hline
\end{tabular}

\section{Fractional Milling Cost Estimation}

Fractional milling cost estimation is based on vendor-supplied information and equipment performance from typical machine performance and process demonstration unit data (Table 52).

Table 52. Fractional milling cost estimates.

\begin{tabular}{|lcc|}
\hline & $\begin{array}{c}\text { 2013 SOT } \\
(2011 \text { \$/dry T })\end{array}$ & $\begin{array}{c}2017 \text { Target } \\
(2011 \text { \$/dry T })\end{array}$ \\
\hline Grinder 1 & Total & Total \\
\hline Separations & 16.80 & 5.10 \\
Grinder 2 & NA & 5.00 \\
Total & 11.60 & 2.40 \\
\hline
\end{tabular}

\subsubsection{Drying and Densification}

\section{Cost Estimation for High-Moisture Densification}

The reduction in drying energy is the key advantage of this approach. First, the process uses the heat generated in the pellet die to partially dry the material. Second, drying the pellets offer cost and energy advantages over drying loose, bulk biomass. Loose biomass typically is dried in a concurrent flow rotary dryer. Rotary biomass dryers typically operate at temperatures of about 150 to $160^{\circ} \mathrm{C}$, have greater particulate emissions, greater volatile organic compound emissions, greater fire hazard, a large footprint, and often have difficulty in controlling the material moisture. With the increased density, the reduced tendency for material to become entrained in the air flow, and the increased heat transfer coefficients compared to loose biomass, more efficient drying technologies options are available for drying pellets. A cross-flow dryer (common in grain drying) operates at temperatures less than $100^{\circ} \mathrm{C}$, reduces the particulate and volatile organic compound emissions, and will have better temperature distribution. A 
comparison of pellet properties and energy balances for conventional and high-moisture pelletization processes is given in Table 53. The table shows 2017 Design Case targets to achieve a 40 to $50 \%$ reduction in the total pelletization and drying energy.

Table 53. Drying and densification design basis

\begin{tabular}{lll}
\hline Moisture Properties & $2013 \mathrm{SOT}$ & 2017 Target \\
\hline Infeed Moisture & $30 \%$ & $30 \%$ \\
Dryer Moisture Reduction & $18 \%$ & $11 \%$ \\
\hline Densification Moisture Reduction & $3 \%$ & $10 \%$ \\
\hline Final Pellet Moisture & $9 \%$ & $9 \%$ \\
\hline Densification Energy & $75 \mathrm{kWhr} / \mathrm{dry} \mathrm{T}$ & $50 \mathrm{kWhr} / \mathrm{dry} \mathrm{T}$ \\
\hline Drying Energy & $350 \mathrm{kWhr} / \mathrm{ton}$ & $100 \mathrm{kWhr} / \mathrm{ton}$ \\
\hline Pellet Properties & & \\
\hline Unit Density & & \\
\hline Bulk Density & $70 \mathrm{lb} / \mathrm{ft}^{3}$ & $65 \mathrm{lb} / \mathrm{ft}^{3}$ \\
\hline Durability & $40 \mathrm{lb} / \mathrm{ft}^{3}$ & $35 \mathrm{lb} / \mathrm{ft}^{3}$ \\
\hline
\end{tabular}

The cost of densification was estimated using vendor-supplied information and the capacity and energy assumptions shown in Table 54. Rotary drying costs associated with the 2013 SOT were based on data supplied by Anco-Eaglin, Inc. As described above, because of the similarity of pellets and grain, grain drying technology is the basis of the 2017 Design Case. Accordingly, grain drying costs also the source of the pellet drying cost estimate. Using a grain drying calculator found at Iowa State ${ }^{49}$, we estimate the cost of drying grain of a similar moisture content to be $\$ 10$ to $\$ 14 /$ ton. Estimated pellet drying costs were reduced from these values because we assume that the porous nature of pellets and less structural heterogeneities in pellets will promote more rapid and uniform drying compared to grain that has the outer pericarp layer that limits moisture transfer.

Table 54. Drying and densification cost estimates.

\begin{tabular}{|c|c|c|}
\hline & $\begin{array}{l}2013 \text { SOT } \\
(2011 \$ / \text { dry T) }\end{array}$ & $\begin{array}{l}2017 \text { Target (2011 } \\
\$ / \text { dry T) }\end{array}$ \\
\hline & Total & Total \\
\hline Drying & 15.20 & 5.60 \\
\hline Densification & 7.70 & 4.40 \\
\hline Totals & 22.90 & 10.00 \\
\hline
\end{tabular}




\subsubsection{Formulation/Blending}

\section{Overview}

Feedstock formulation is not a new concept in many market sectors. For example, different grades of coal are blended to reduce sulfur and nitrogen contents for power generation ${ }^{14}$, grain is blended at elevators to adjust moisture content ${ }^{13}$, animal feeds are blended to balance nutrient content ${ }^{16}$, and high-ash biomass sources are mixed with low-ash coal to allow their use in biopower ${ }^{17}$. However, blending/formulation is not part of the baseline design.

\section{Formulation Design Basis}

To meet feedstock specifications required for various conversion pathways, formulation of specific mixtures of feedstocks will likely be required. Examples include mixing high and lowcost feedstocks to meet cost targets, mixing high and low-ash feedstocks to meet an ash target, mixing of high and low-carbohydrate feedstocks to meet a yield target, and mixing easily and poorly reactive feedstocks to meet a convertibility target. An example of blending to meet an ash and moisture specification is shown in Table 55.

Table 55. Feedstock formulation/blending of ash and moisture contents*.

\begin{tabular}{|lccccc|}
$\begin{array}{l}\text { Content Delivered to } \\
\text { Biorefinery Infeed }\end{array}$ & $\begin{array}{c}\text { Single pass } \\
\text { cornstover(35\%) }\end{array}$ & $\begin{array}{c}\text { Multi-pass } \\
\text { cornstover( } \\
\mathbf{2 5 \%}\end{array}$ & $\begin{array}{c}\text { Switchgrass( } \\
\mathbf{3 5 \%}\end{array}$ & $\begin{array}{c}\text { MSW( } \\
\mathbf{5 \%}\end{array}$ & $\begin{array}{c}\text { Final } \\
\text { Blend }\end{array}$ \\
\hline $\begin{array}{l}\text { Ash content (wt. \%) } \\
\begin{array}{l}\text { Moisture content } \\
\text { (\%, wet basis) }\end{array}\end{array}$ & 3.5 & 7 & 4 & 10 & $\mathbf{4 . 9}$ \\
$\begin{array}{l}\text { Carbohydrate content (wt. } \\
\text { \%) }\end{array}$ & 64 & 9 & 9 & 9 & $\mathbf{9}$ \\
\hline
\end{tabular}

Assumptions for the formulation design basis are as follows:

- Blended feedstocks will be selected and developed to achieve conversion yield specifications. It is currently unknown how blended feedstocks will perform in the conversion pathways. The simplest assumption is that the performance of the blended feedstocks would be the sum of performances of each individual component. However, two, small-scale studies demonstrated that the performance of blended feedstocks ranged from under to over performance, depending on the conditions assessed. In the first study, ${ }^{50}$ examined a blend of wheat straw, barley straw, hardwood, and softwood subjected to three different types of pretreatments: dilute acid, lime, and soaking in aqueous ammonia. After pretreatment, the feedstocks were hydrolyzed using commercial cellulose enzymes ${ }^{51}$ and sugar yields were measured. Ethanol yields also were determined using simultaneous saccharification and fermentation ${ }^{52}$.

- For the dilute acid and soaking in aqueous ammonia treatments, the yields of C6 sugars were lower than would be predicted by simple summation, while the C6 sugar yield was slightly higher than predicted for the lime treatment. However, the opposite trends were observed for ethanol production, with higher ethanol production for dilute acid and soaking in aqueous ammonia and lower production for lime treatment. It is not clear from the report whether or not these differences were statistically significant. It also was shown that yields of both C6 
sugars and ethanol were lower than predicted for non-optimized pretreatments. This may indicate that the pretreatment has to be optimized for the most recalcitrant component, which may lead to formation of sugar degradation products and fermentation inhibitors. In the second study, ${ }^{53}$ examined a mixture of corn stover, switchgrass, eucalyptus, and lodgepole pine. This mixture was pretreated with an ionic liquid (i.e., 1-ethyl-3-methylimidazolium acetate) and the resulting sugars measured. The mixed feedstock released more glucose than would be expected from the sum of the individual feedstocks.

- Individual feedstocks will be pelleted at depots for shipment to biorefineries. At the biorefinery, the pelleted feedstocks will be unloaded and conveyed into individual bunkers for storage. Pellets of the different blendstocks will be metered out into the bunkers in the ratios required of the blends, crushed (using a pellet crusher), and then mixed prior to insertion into the conversion process.

- Material will be metered from individual bunkers onto a conveyer and will be thoroughly homogenized during this process with no segregation. Mixing of solids occurs in many industries and is often problematic when solids of varying density, shape, and size are blended. This often leads to segregation, either during the mixing or while being transported to its destination. Mixing of solids is considered a trial-and-error process due to these issues.

- The expected unit operations for formulation are shown in Table 56.

Table 56. Feedstock formulation design basis.

\begin{tabular}{|lcc|}
\hline 2013 SOT $(2011 \$)$ & \multicolumn{2}{c}{ Operating Parameters } \\
\hline Pellet Pulverizer & 100 dry T/hour & Horsepower \\
\hline Bulk Storage with Hopper & 30 dry T/hour & $200 \mathrm{HP}$ \\
\hline Conveying/Mixing System & 30 dry T/hour & $40 \mathrm{HP}$
\end{tabular}

Research currently is ongoing at INL to examine the compatibility of various feedstocks in formulated blends, with an initial focus on the reactivity of blends versus the individual feedstocks. Blends will be developed for several regions of the United States using the least-cost formulation model as a starting point and will incorporate feedstocks with varying levels of reactivity (e.g., herbaceous, woody, and MSW). Reactivity for the fermentation pathway will be investigated first, with expansion into the other DOE conversion pathways in later fiscal years. Reactivity for the fermentation conversion pathway will be measured as production of sugars using dilute acid pretreatment followed by enzymatic hydrolysis. Production of sugar decomposition products and other inhibitors also will be monitored. Hydrolysis conditions will be optimized for each feedstock and then each of the optimum conditions used on the formulated feedstock. Research is planned to examine mixing issues associated with blended feedstocks. A survey of current state-of-the-art mixing technologies will be conducted, and those technologies relevant to feedstocks will be further examined to determine the best technology to ensure thorough homogenization without segregation. 
While the costs for preprocessing of herbaceous feedstocks (e.g., grinding, chemical preconversion, pelleting, and drying) are addressed in other parts of the 2017 Design Case, MSW will require a different set of preprocessing options to produce a stable, high-quality feedstock.

\section{Cost Estimation for Formulation}

Formulation cost estimation was based on existing technology, vendor-supplied information and equipment performance (Table 57).

Table 57. Formulation cost estimation

\begin{tabular}{|lc|}
\hline & 2017 Target $(2011$ \$/dry T) \\
\hline Pellet pulverizer & 1.10 \\
\hline Bulk storage with hopper & 0.20 \\
\hline Conveying/Mixing system & 0.50 \\
\hline Totals & $\mathbf{1 . 8 0}$ \\
\hline
\end{tabular}

\subsubsection{Transportation and Handling}

The 2017 Design Case includes formulation and densification to meet feedstock specifications and costs targets. Both of these active processes will improve feedstock handling operations through active controls. Given formulation and the specific quantities of individual feedstocks required, the average transportation distance (and even mechanism) will change based on feedstock type. In the 2017 Design Case, corn stover will be trucked from a local draw radius of about 25 miles (compared to 35 miles) while switchgrass will be trucked 15 miles. MSW will need to be transported from a larger metropolitan area to obtain the required quantities; therefore, it will be transported by rail (either by unit train or single car) from as far as 200 miles away. Corn stover and switchgrass will be loaded and unloaded at each location using a telehandler capable of moving $12-\mathrm{lb} / \mathrm{ft}^{3}$ bales at 30 and $20 \%$ respective moisture contents. A $53-\mathrm{ft}$ trailer and 800,000-GVW limits were assumed in all trucking operations. Transportation for corn stover and switchgrass will occur from a field side stack to a densification facility completely separate from the conversion location, but is within a minimal conveyor distance. MSW transportation will occur from the waste transfer station to a densification facility. Further transportation and handling assumptions are given as follows:

- At $30 \%$ moisture, transportation of cornstover continues to be volume limited due to low densities $\left(12 \mathrm{lb} / \mathrm{ft}^{3}\right)$

- At $20 \%$ moisture, transportation of switchgrass continues to be volume limited due to low densities $\left(12 \mathrm{lb} / \mathrm{ft}^{3}\right)$

- There will be insignificant material losses throughout transportation and handling.

- Densification will increase material uniformity and flowability. 


\subsubsection{Cost Estimation for Transportation}

The cost estimation for transportation and handling was based on vendor-supplied information and equipment performance from typical machines (Table 58). Rail transportation costs were estimated using a jumbo hopper car ${ }^{54}$ adjusted for U.S. conditions.

Table 58. Transportation cost estimates

\begin{tabular}{|c|c|c|}
\hline & $\begin{array}{c}2013 \text { SOT } \\
(2011 \$ / \text { dry T) }\end{array}$ & $\begin{array}{c}2017 \text { Target (2011 } \\
\text { \$/dry T) }\end{array}$ \\
\hline & Total & Total \\
\hline Truck & 11.50 & 8.30 \\
\hline Rail* & 0.00 & 18.00 \\
\hline $\begin{array}{l}{ }^{*} \text { For s } \\
\text { quantity }\end{array}$ & feedstocks on & obtain required \\
\hline
\end{tabular}

\subsection{Life Cycle Assessment:}

This section uses a life-cycle assessment (LCA) to evaluate the energy input and GHG emissions for Biological Conversion of Sugars to Hydrocarbons. LCA considered the unit processes involved in the biomass logistics. Table 59 shows the energy consumption and Table 60 shows GHG contribution for Biochemical conversion supply chain design.

Table 59. Energy consumption for Biochemical conversion supply chain design.

\begin{tabular}{|lcccc|c|}
\multicolumn{1}{c}{ Process Element } & $\begin{array}{c}\text { Single-pass } \\
\text { Corn Stover }\end{array}$ & $\begin{array}{c}\text { Multi-pass } \\
\text { Corn Stover }\end{array}$ & Switchgrass & MSW & Blend \\
\hline $\begin{array}{l}\text { Formulation Contribution } \\
\text { Harvest and Collection }\end{array}$ & $\mathbf{3 5 \%}$ & $\mathbf{2 5 \%}$ & $\mathbf{3 5 \%}$ & $\mathbf{5 \%}$ & - \\
$\begin{array}{l}\text { (MBTU/dry T) } \\
\text { Transportation (MBTU/ dry }\end{array}$ & 142.08 & 142.08 & 99.35 & 549.43 & $\mathbf{1 4 7 . 4 9}$ \\
$\begin{array}{l}\text { T) } \\
\text { Preprocessing (MBTU/dry }\end{array}$ & 315.82 & 315.82 & 285.83 & 421.25 & $\mathbf{3 1 0 . 6 0}$ \\
$\begin{array}{l}\text { T) } \\
\text { Storage (MBTU/dry T) }\end{array}$ & 21.83 & 21.83 & 21.83 & 21.83 & $\mathbf{2 1 . 8 3}$ \\
\hline $\begin{array}{l}\text { Handling (MBTU/dry T) } \\
\text { Total Energy Consumption }\end{array}$ & $\mathbf{4 1 . 9 0}$ & 41.90 & 41.90 & 41.90 & $\mathbf{4 1 . 9 0}$ \\
Cost (MBTU/dry T) & $\mathbf{6 3 0 . 3 2}$ & $\mathbf{7 2 5 . 8 1}$ & $\mathbf{5 7 1 . 7 6}$ & $\mathbf{1 0 3 4 . 4 1}$ & $\mathbf{6 5 3 . 9 0}$
\end{tabular}


Table 60. GHG contribution for biochemical conversion supply chain design.

\begin{tabular}{|c|c|}
\hline Process Element & Blend \\
\hline Formulation Contribution & - \\
\hline $\begin{array}{l}\text { Harvest and collection } \\
\text { GHGs }\left(\mathrm{Kg} \mathrm{CO}_{2} \mathrm{e} / \text { dry T) }\right.\end{array}$ & 10.9 \\
\hline $\begin{array}{l}\text { Transportation GHGs }\left(\mathrm{Kg} \mathrm{CO}_{2} \mathrm{e} / \mathrm{dry}\right. \\
\mathrm{T})\end{array}$ & 8.62 \\
\hline $\begin{array}{l}\text { Preprocessing GHGs ( } \mathrm{Kg} \mathrm{CO}_{2} \mathrm{e} / \mathrm{dry} \\
\mathrm{T})\end{array}$ & 27.02 \\
\hline Storage GHGs $\left(\mathrm{Kg} \mathrm{CO}_{2} \mathrm{e} / \mathrm{dry} \mathrm{T}\right)$ & 6.42 \\
\hline Handling GHGs ( $\mathrm{Kg} \mathrm{CO}_{2} \mathrm{e} /$ dry T) & 6.41 \\
\hline Total GHGs (Kg CO $2 \mathrm{e} /$ dry $\mathbf{T})$ & 59.4 \\
\hline
\end{tabular}




\section{Conversion of Lignocellulosic Biomass to Hydrocarbon Fuels: Thermochemical Pathways with In Situ and Ex Situ Upgrading of Fast Pyrolysis Vapors}

This sections is intended to couple with the National Renewable Energy Laboratory's (NREL's) hydrocarbon design report, "Process Design and Economics for the Conversion of

Lignocellulosic Biomass to Hydrocarbon Fuels: Thermochemical Pathways with In Situ and Ex Situ Upgrading of Fast Pyrolysis Vapors ${ }^{6}$ that describes a viable route from biomass to hydrocarbon fuels. The assumptions of scale and feedstock quality requirements are consistent with the design case assumptions used by NREL in their report and techno- economic assessments. This design does not consider the different requirements and nuances of other thermochemical conversion processes or other hydrocarbon pathways.

\subsection{Summary}

This report establishes a plausible case for achieving the 2017 Design Case for Fast Pyrolysis conversion to bio-oils cost goals of delivering a biomass feedstock to the conversion facility at a cost of $\$ 80 /$ dry $T$ (Table 61). The least-cost formulation approach (Appendix B) illustrates the importance of cost estimates for determining the total cost of feedstock to a biorefinery, including grower payment (access costs), logistics costs, and quality/dockage cost. It also illustrates the importance of refining and updating these costs as analyses and data improve to better inform the estimates. The following conclusions are presented to document the specific areas that require additional attention to further strengthen and support the feedstock design detailed in this report. 
Table 61. Thermochemical feedstock design cost analysis for 2017 .

\begin{tabular}{|c|c|c|c|c|c|}
\hline Cost Element & Pulpwood & Wood Residues & Switchgrass & $\begin{array}{l}\text { Construction and } \\
\text { Demolition Waste } \\
\text { (C\&D) }\end{array}$ & Blend \\
\hline Formulation Contribution & $45 \%$ & $32 \%$ & $3 \%$ & $20 \%$ & - \\
\hline $\begin{array}{l}\text { Grower payment/ } \\
\text { access cost }\end{array}$ & 25.00 & 26.35 & 19.67 & 8.15 & 21.90 \\
\hline $\begin{array}{l}\text { Harvest and Collection } \\
\text { (\$/dry T) }\end{array}$ & 22.24 & 0 & 15.41 & - & 10.47 \\
\hline $\begin{array}{l}\text { Landing Preprocessing/ } \\
\text { Sorting (\$/dry T) }\end{array}$ & 12.17 & 8.73 & 0 & 9.85 & 10.24 \\
\hline Transportation (\$/dry T) & 10.89 & 3.33 & 4.5 & 6.87 & 7.48 \\
\hline Preprocessing (\$/dry T) & 23.97 & 23.97 & 19.7 & 28.12 & 24.67 \\
\hline Storage (\$/dry T) & 3.23 & 3.23 & 5.5 & 3.23 & 3.30 \\
\hline Handling (\$/dry T) & 1.90 & 1.90 & 1.90 & 1.90 & 1.90 \\
\hline $\begin{array}{l}\text { Total Delivered Feedstock } \\
\text { Cost }(\$ / \text { dry } T)\end{array}$ & 99.49 & 67.51 & 66.68 & 58.12 & 80.00 \\
\hline \multicolumn{6}{|c|}{ Delivered Feedstock Specifications } \\
\hline Ash content (wt. \%) & 0.5 & 1.0 & 4.0 & 1.0 & $<1 \%$ \\
\hline $\begin{array}{l}\text { Moisture content } \\
\text { (\%, wet basis) }\end{array}$ & 9 & 9 & 9 & 9 & 9 \\
\hline HHV (BTU/lb) & 8824 & 9444 & 7557 & 8824 & 8984 \\
\hline LHV (BTU/lb) & 7255 & 7616 & 6155 & 7255 & 7337 \\
\hline
\end{tabular}

Continued refinements of the biomass supply curves to represent the latest estimates for biomass grower payment are needed to support the least-cost formulation approach. Ultimately, translating The Billion Ton Update 5 data from farm gate price to grower payment is necessary to establish better grower payment estimates. The grower payment estimates included in this report were calculated by subtracting our harvest and collection costs from the farm gate price.

Logistics costs are based on actual field trial data but do not include the cost of various business elements, such as profit margins for transportation, depots and field agents that would be involved throughout a biomass feedstock supply chain. This would increase the overall cost of the supply system than is demonstrated in this report. This was of little consequence to the 2012 Conventional Design Case target that intentionally focused only on logistics costs. The 2017 Design Case, on the other hand, is meant to encompass total delivered feedstocks costs. Further, the complexity of a blended feedstock approach may introduce multiple business elements into the supply chain; therefore, it is important that logistics costs be updated to include the true cost of these business elements, including a return on investment.

As the biomass logistic systems become more complex, especially with the introduction of new technologies (e.g., chemical preconversion), it may be prudent to differentiate between the current state-of-technology costs and the projected costs of mature technology ( $\mathrm{n}^{\text {th }}$ plant costs) to 
be consistent with conversion platform terminology. This was not an issue with conventional feedstock designs that were intrinsically tied to current SOT; however, for technology maturation, cost reductions may be worth considering for advanced feedstock designs.

Admittedly, it also is necessary to tighten the design and cost estimates around formulation and the engineering systems for crushing the pellets and blending prior to insertion into the conversion process. A better understanding of C\&D availability, cost, and conversion performance is needed to solidify its position in the 2017 Design Case. Likewise, the viability of blended feedstocks as a whole depends on their conversion performance. DOE Bioenergy Technology Office funded research is investigating the conversion performance of blends (including C\&D blends) and evaluating the compatibilities and incompatibilities of blendstocks. The results of this research are critical to further development of blended feedstocks.

\subsection{Feedstock Composition (In-feed quality specifications)}

The dry basis elemental composition of the feedstock, shown in Table 62, is similar to previous NREL and PNNL design reports ${ }^{6}$. The composition was originally assumed to come from pulpwood. Recent feedstock logistics work suggests the use of blended material may be required to meet a cost target of $\$ 80 /$ dry US ton while still meeting these specifications. For the purpose of this report, it is assumed that any blended material provided to meet this feedstock elemental composition will not adversely affect fast pyrolysis conversion efficiencies. Ongoing studies being conducted jointly by INL, NREL and PNNL will provide experimental evidence of the impact of blended feedstocks on fast pyrolysis and gasification processes. Future TEA will be modified to reflect conversion impacts inferred from such studies. As has been described in prior conversion design reports, the feedstock composition plays a critical role on overall process design and economics, primarily with respect to high and low heating values, carbon as well as high sensitivity to impurity components such as ash and metals in this design. The blended uniform-format feedstock composition assumed here for purposes of future design case targets is shown below, with supporting details described in the 2011 ethanol report ${ }^{46}$. Also consistent with prior design cases, the moisture content for the delivered feedstock is $10 \%$ or less. 
Table 62. Delivered woody feedstock composition and processing assumptions for the In Situ and Ex Situ Upgrading of Fast Pyrolysis Vapors ${ }^{6}$.

\begin{tabular}{|lc|}
\hline Component & $\begin{array}{c}\text { Composition } \\
\text { (dry wt. \%) }\end{array}$ \\
\hline Carbon & 50.94 \\
\hline Hydrogen & 6.04 \\
\hline Nitrogen & 0.17 \\
\hline Sulfur & 0.03 \\
\hline Oxygen & 41.90 \\
\hline Ash & $0.90-1.0$ \\
\hline Heating Value (Btu/lb) & $8,601 \mathrm{HHV}$ \\
\hline & $7,996 \mathrm{LHV}$ \\
\hline Moisture (Bulk Wt. \%) & 10.0 \\
\hline Particle Size (inch) & $1 / 4$ \\
\hline
\end{tabular}

\subsection{Feedstock Selection Cost Estimation}

Expansion beyond highly productive resource areas has significant implications to the feedstock supply chain. Sparse areas, whether due to reduced yields and/or higher dispersion, typically increase feedstock logistics costs. Higher harvest and collection costs are incurred due to the need to spread machinery ownership costs over fewer tons of biomass or the need to cover more acres for the same quantity of biomass. Additionally, lower resource yields increase the supply radius and biomass transportation distances. Under the 2012 Conventional Design, higher yield areas allow refinery to be selective on the resource that they access.

Consider, for example, the scenarios depicted in Figure 39. This resource map illustrates a county-level resource assessment of pulpwood farm gate at $\$ 60 /$ dry T prices (this includes grower payment, harvest, collection, and chipping costs). Farm gate price data were extracted from The Billion Ton Update (BT2) ${ }^{5}$ data supplied from Oak Ridge National Laboratory. It should be noted that while the data is reported at a county-level, the data should be applied at the wood shed (typically much larger area than a county) level because it was derived from the U.S. Forest Service Forest Inventory and Assessment Data (FIA) ${ }^{56}$ and does not equate to county levels accurately. The FIA is a woodshed level assessment and therefore to use the data correctly it is necessary to combine multiple counties.

The cost competitiveness of the 2012 Conventional Design was demonstrated in the scenario located in southern Alabama, a high biomass yielding area. We further suggest, based on the consistency of farm gate (i.e., landing) prices shown in this map, that the 2012 Conventional Design can be deployed cost effectively in South Carolina. Commercial readiness of conventional supply systems ultimately will be demonstrated by commercial-scale cellulosic ethanol plants opening in these areas in the near future. 


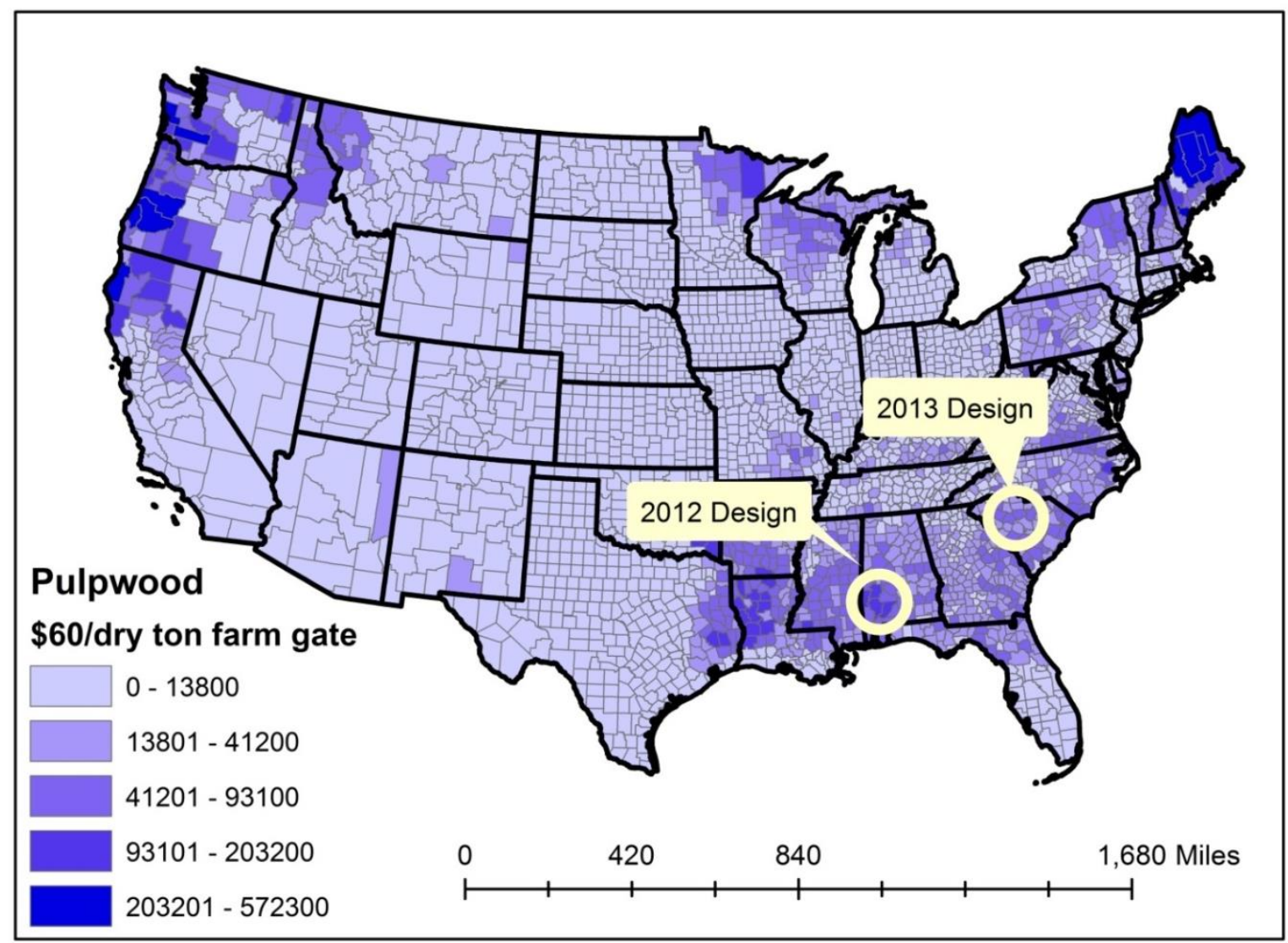

Figure 39. Total tons per county of available pulpwood at $\$ 60 /$ dry $\mathrm{T}$ farm gate price. Yellow circles show areas represented in the 2012 Conventional Design and the Relocated (2013) Design Case ${ }^{5}$.

Access costs are calculated from the grower payment cost curves shown in Figure 6, which are derived from historical prices. The 2017 Design Case basis discussion presented above provided the least-cost formulation approach for reducing access costs by accessing multiple feedstocks. With this approach, reduced quantities of each feedstock allows us to stay lower on the supply curve than if we had to supply the entire refinery with any single feedstock. The impact of this approach is shown in Table 63. The 2013 State of Technology assumes a 100\% supply of pulpwood of 909,100 dry $\mathrm{T}$ at an estimated $\$ 60 /$ dry $\mathrm{T}$ farm gate or a $\$ 25 /$ dry $\mathrm{T}$ access cost. In comparison, the 2017 Design Case blend of 45\% pulpwood, 32\% wood residues, 20\% C \& D waste, and 3\% switchgrass results in a weighted average feedstock cost that is nearly $15 \%$ lower than the access cost of pulpwood alone. 
Table 63. Resource access cost estimate (U.S. DOE $2011^{5}$, and INL MSW Data).

\begin{tabular}{lcccc} 
& \multicolumn{2}{c}{ 2013 SOT } & \multicolumn{2}{c}{ 2017 Target } \\
& $\begin{array}{c}\text { Access Cost } \\
(2011 \text { \$/dry T) }\end{array}$ & Tons & $\begin{array}{c}\text { Access Cost } \\
(2011 \$ / \text { dry T })\end{array}$ & Tons \\
Pulpwood & 25.00 & $909,100^{*}$ & 25.00 & $425,700^{*}$ \\
Wood Residues & NA & NA & 26.35 & $412,800^{* *}$ \\
Switchgrass & NA & NA & 19.67 & 25,800 \\
C\&D & NA & NA & 8.15 & 172,000 \\
Totals & 25.00 & NA & 21.90 & $1,036,300$
\end{tabular}

*assumes $10 \%$ loss of material to debark/delimb ${ }^{18}$.

** assumes $40 \%$ loss of material to clean up residues ${ }^{19}$.

\subsection{Quality Specification and Design Assumptions}

The 2012 Conventional Design focused on conventional woody harvest operations (i.e., felling and/ or skidding operations are separate from landing preprocessing operations). In addition to including switchgrass into the feedstock blend, the 2017 Design Case assumes that the immaturity of the biomass market will limit the forest and farm owner's investment in advanced equipment options for both woody and herbaceous feedstocks. Therefore, with the exception of a few proactive, early adopters, conventional forestry and farming operations will dominate the market in the regions defined by the 2017 Design Case. Table 64 below summarizes the assumptions and differences between the 2012 and 2017 logistic designs. 
Table 64. Summary of assumptions underpinning progressive design implementations ${ }^{57}$.

\begin{tabular}{|c|c|c|c|}
\hline & $\begin{array}{l}2012 \\
\text { Conventional } \\
\text { Design }\end{array}$ & Baseline & 2017 Design Case \\
\hline Feedstock(s) & Pulpwood & Pulpwood & $\begin{array}{l}\text { Blended feedstock: pulpwood }(45 \%) \text {, wood } \\
\text { residues }(32 \%) \text {, switchgrass }(3 \%) \text {, and select } \\
\text { construction and demolition wastes (C } \\
\& D)(20 \%)\end{array}$ \\
\hline $\begin{array}{l}\text { Grower } \\
\text { payment }\end{array}$ & $\begin{array}{l}\text { Breakeven cost of } \\
\text { production }\end{array}$ & $\begin{array}{l}\text { Increases based } \\
\text { on marginal } \\
\text { cost differential }\end{array}$ & $\begin{array}{l}\text { Calculated and modeled according to specific } \\
\text { location and resource blend/formulation }\end{array}$ \\
\hline Moisture & Field dried to $40 \%$ & $\begin{array}{l}\text { Field dried to } \\
40 \%\end{array}$ & $\begin{array}{l}\text { Arrives: Pulpwood chips } 30 \% \text { wood residue } \\
\text { chips } 30 \% \text {, switchgrass } 20 \% \text {, and C\& D } \\
\text { ground } 20 \% \text {; } \\
\text { All dried to } 9 \% \text { pellets }\end{array}$ \\
\hline Ash & Debark/delimb & Debark/delimb & $\begin{array}{l}\text { Debark/delimb pulpwood } \\
\text { Trommel screen residues } \\
\text { Wash and sort C\& D waste } \\
\text { Blended ash content of }<1 \% \\
\text { Debarked pulpwood }<1 \% \text {, screened wood } \\
\text { residues } 1.4 \% \text {; washed and sorted C\&D } 1.0 \%\end{array}$ \\
\hline Logistics & $\begin{array}{l}\text { Uses existing } \\
\text { systems }\end{array}$ & $\begin{array}{l}\text { Uses existing } \\
\text { systems }\end{array}$ & $\begin{array}{l}\text { Pneumatics attached to hammer mill } \\
\text { High-moisture densification }\end{array}$ \\
\hline $\begin{array}{l}\text { Quality } \\
\text { controls } \\
\text { (passive) }\end{array}$ & $\begin{array}{l}\text { Field drying to } \\
\text { reduce moisture } \\
\text { Ample available } \\
\text { resource; quality } \\
\text { spec manually } \\
\text { selected }\end{array}$ & $\begin{array}{l}\text { Field drying to } \\
\text { meet moisture } \\
\text { spec }\end{array}$ & $\begin{array}{l}\text { Harvest/collection and storage best management } \\
\text { practices for pulpwood and switchgrass } \\
\text { More rigorous field drying of pulpwood and } \\
\text { residues }\end{array}$ \\
\hline $\begin{array}{l}\text { Quality } \\
\text { controls } \\
\text { (active) }\end{array}$ & Waste heat dryer & Rotary drying & $\begin{array}{l}\text { Multiple resource blending/formulation } \\
\text { High-moisture densification } \\
\text { High-efficiency pellet drying }\end{array}$ \\
\hline $\begin{array}{l}\text { Meets quality } \\
\text { target }\end{array}$ & Yes & Yes & Yes \\
\hline $\begin{array}{l}\text { Meets cost } \\
\text { target }\end{array}$ & Yes & No & Yes \\
\hline $\begin{array}{l}\text { Accesses } \\
\text { dispersed } \\
\text { resources }\end{array}$ & No & No & Yes \\
\hline
\end{tabular}


Relative to the woody feedstocks used in the Thermochemical Design Case, the 2013 baseline and 2017 Design Case are similar in many ways for harvest and collection, but the latter has two key changes to improve quality and production of woody materials. While each system is discussed later, the key differences of the 2017 Design Case are first inclusion of woody residues sourced from pulpwood operations, and second in-forest drying of whole tree piles at the landing to achieve a more aggressive moisture content of 30\%.In this design debarking and delimbing are conducted to improve biomass quality. Construction and demolition wastes are considered to enter the feedstock logistics system at the preprocessing stage and are therefore not discussed here.

\subsection{Feedstock Logistics}

\subsubsection{Harvest and Collection}

Conventional wood harvest and collection relies on existing forestry technologies designed for timber and pulp and paper production. Collection systems for woody material involve cutting the feedstock with a tracked feller buncher and transporting the material to the landing with a grapple skidder immediately after felling. Felling and skidding operations increase the overall ash content of harvested whole-trees by introducing soil as it is moved in contact with the ground from one location to the next ${ }^{58}$. In Situ and Ex Situ Upgrading of Fast Pyrolysis Vapors are highly sensitive to silica, alkali, and transition metals found in most soils, impacting process performance $^{596061}$. In both the 2013 and 2017 Design Cases active management strategies are employed during the landing preprocessing to improve biomass ash content .Current forestry production of pine pulpwood is reported to yield roundwood with a moisture content of 45-55\% and a whole-tree ash content ranging from 1\% to 3\% ${ }^{62} 6364$. The 2013 baseline assumes the moisture content of pulpwood to be $50 \%$ for whole-trees entering storage.

Woody residues are generated through typical commercial forestry operations on southern pine plantations where trees are harvested for pulpwood, chip-and-saw, and saw timber. Similar to the above described collection of pulpwood, these operations bring whole trees to the landing where they are delimbed and topped using a pull-through delimber. The roundwood is then loaded onto trucks for delivery to the mill while the residues are piled at the landing. While not collected in the 2013 baseline, the 2017 Design Case utilizes these materials as a fraction of the feedstock blend. The baseline for residue moisture content is reported at $40 \%$, while ash content has been reported to range from $2 \%$ to $4 \% 47,62,6566$. Switchgrass, which is part of the blend, harvest and collection systems use a conventional windrowing harvester and rectangular baler $(3 \times 4 \times 8-\mathrm{ft})$.

\subsubsection{Harvest and Collection Design Basis}

The 2017 Design Case incorporates a chain flail debarker during preprocessing at the landing to increase the quality of the final chipped pulpwood product. Therefore, moisture and ash contents of material entering storage are not different between the 2013 and 2017 Designs. However, there is still impetus to increase the operational efficiency of roundwood collection for reducing costs ${ }^{6768}$.This can be achieved through forest management shifts to short-rotation pine plantations aimed at supplying bioenergy production, increased efficiency of harvesting machinery, and increased efficiency of grapple skidder transportation. Research conducted by Auburn University for the DOE High Tonnage Forest Biomass Project has demonstrated high capacity grapplers to increase productivity by 80 tons per productive machine hour compared to 
traditional systems ${ }^{58}$. Figure 40 depicts a conventional skidder and a high capacity skidder. Further development of such operational improvements will play a key role in reducing costs of clean pulp chips for thermochemical conversion. In addition, transition of forest management to short rotation pine plantations focused on energy use is a promising option for increasing yields; should the economics of establishment be overcome ${ }^{69}$.

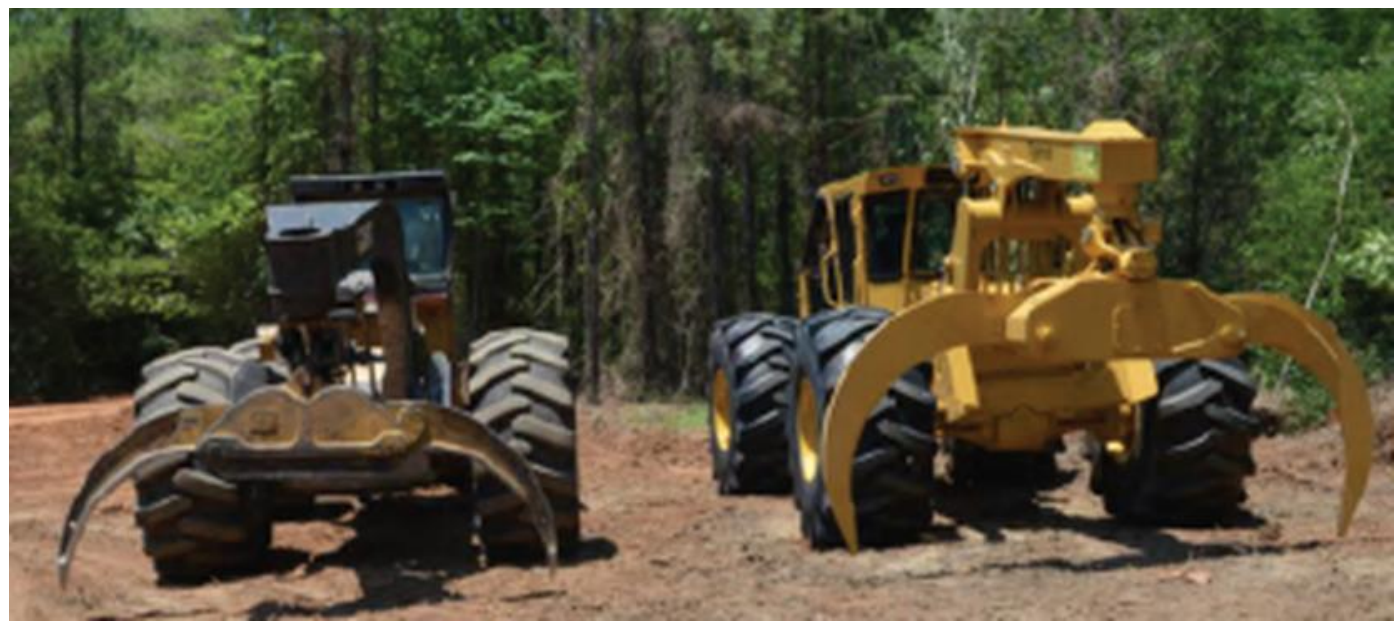

Figure 40. Conventional (left) and high-capacity grapple skidder (right) for transporting small diameter pulpwood from the forest to the landing. Photo credit: Auburn University High Tonnage Forest Biomass Project ${ }^{58}$.

Wood residues (tree tops and limbs) originate from other commercial logging operations and are located in piles at the landing, eliminating the costs for harvest and collection (e.g., felling and skidding). Similar to pulpwood, the 2017 Design Case incorporates active quality controls to reduce the ash content during preprocessing at the landing. These active controls applied after storage may contain ash contents in excess of the desired specification of $0.9 \%$ for wood residues and less for pulpwood.

Switchgrass harvest in the 2017 Design Case follows conventional practices for feed and forage in terms of the equipment used, but incorporates more rigorous passive quality controls to reduce ash content. Delayed harvest of switchgrass provides the benefits of reducing moisture and ash content, but even with the practice of delayed-harvest, it is clear that the raw feedstock will not meet the final quality specification for ash. Blending of switchgrass with a low-ash feedstock is necessary to achieve ash specification of $<1 \%$. Nevertheless, it is important that best management practices for switchgrass harvest are used to reduce soil contamination during the processes of cutting and baling while respecting the relationship between delayed harvest date and collection efficiency. Research conducted by Oklahoma State University in collaboration with INL shows that switchgrass can achieve moisture contents at or below the 2017 Design Case specification ( $10 \%$ to $5 \%$ ), though climatic variance can still introduce moisture variability in delayed harvests (Figure 41). In this same research the ash content of switchgrass was found to be low even at an early harvest (5\% in August), though a decreasing trend was observed as harvest was delayed ( $4 \%$ by December). This work stands as an example of the effectiveness of proper harvesting techniques, and stresses the importance of establishing best management 
practices to cope with variability in weather conditions. Goals for the 2017 Design Case include reducing ash content to $4 \%$ through harvest timing and advanced harvesting techniques.

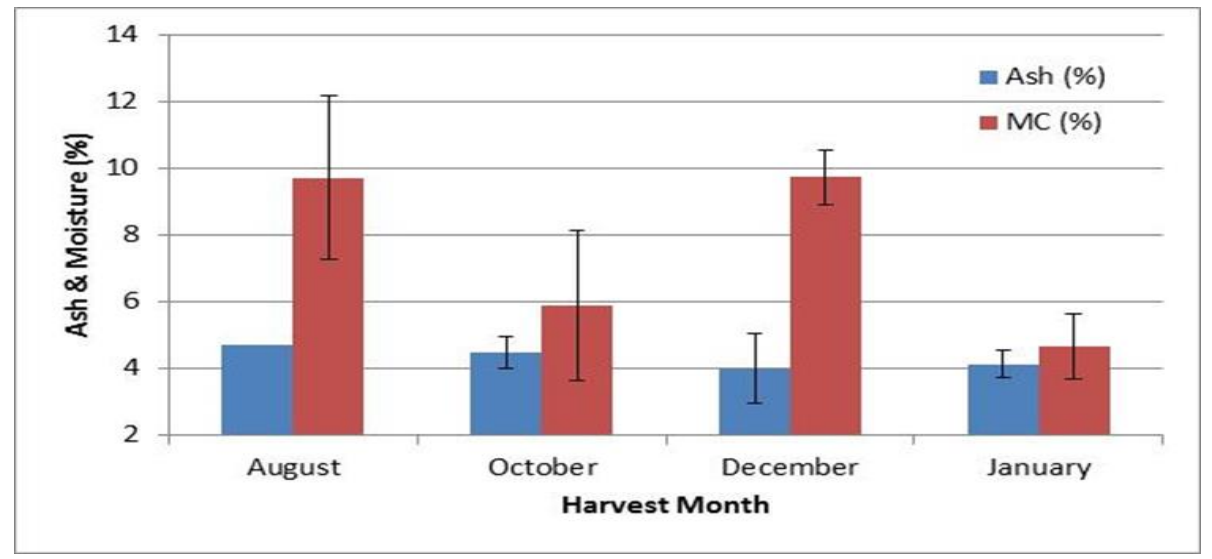

Figure 41. Ash and moisture content of switchgrass harvested in Oklahoma, 2010 by Oklahoma State University. Error bars represent one standard deviation. Ash samples for October, December, and January are three samples comprised of six individual core samples composited.

\subsubsection{Harvest and Collection Cost Estimation}

Harvest and collection costs assume a removal rate of 15 dry T/acre for pulpwood ${ }^{70}, 4$ dry T/acre for residues ${ }^{66}$, and 5-dry T/acre for switchgrass ${ }^{71}$. These assumptions are consistent with those used in the Billion Ton Update ${ }^{5}$. Cost of harvest and collection is shown in Table 65 . The cost of ash at this point within the feedstock logistics system described in section 4 is not yet applicable to pulpwood or wood residue, as the material will undergo active quality controls during landing preprocessing. Switchgrass may be subjected to an ash dockage at this point in the process if ash contents are greater than those needed by the feedstock blending process. 
Table 65. Biomass harvest and collection cost estimates derived from INL analysis.

\begin{tabular}{|lll|}
\hline Machine & $\begin{array}{l}2013 \text { SOT } \\
(2011 \$ / \text { dry T) }\end{array}$ & $\begin{array}{l}2017 \text { Target }(2011 \\
\text { \$/dry T) }\end{array}$ \\
\hline $\begin{array}{l}\text { Total } \\
\text { Pulpwood }\end{array}$ & Total \\
\hline Felling & 15.00 & 15.00 \\
\hline Yarding & 7.24 & 7.24 \\
Totals & 22.24 & 22.24 \\
\hline Wood Residues* & & \\
\hline Felling & 0.00 & 0.00 \\
Yarding & 0.00 & 0.00 \\
Totals & 0.00 & 0.00 \\
Switchgrass & & \\
Mower-conditioner & 4.80 & 4.80 \\
Baler & 7.30 & 7.30 \\
Bale collection/stacking & 3.30 & 3.30 \\
Totals & 15.40 & 15.40 \\
* Costed to harvest and collection of other process.
\end{tabular}

\subsubsection{Storage}

\subsubsection{2013 State of Technology}

Because the 2017 Design Case utilizes a blended feedstock, switchgrass storage must be addressed. The storage of switchgrass occurs field side or at a similar on-farm unimproved storage site. As for any baled feedstock, appropriate storage sites provide adequate drainage away from the stack to prevent the accumulation of moisture around the stack, provide yearround access, and preferably allow stack to be positioned in a North-South orientation to reduce moisture accumulation on the north side of the stack ${ }^{72}$. Tarped stacks are chosen as a balance between bale protection against moisture infiltration, which leads to dry matter loss, and storage configuration costs ${ }^{73}$ 22. Stacks are constructed with a self-propelled stacking bale wagon and are six bales high and covered with a high-quality hay tarp. In order to prolong tarp life, it is also important that adequate year-round maintenance be provided to periodically tighten the tarps ${ }^{74}$. Biomass storage systems in the current Design Case seek to provide a low-cost, lowmaintenance, moisture-tolerant solution that focus on maintaining moisture content $<20 \%$, minimizing dry matter loss and preserving feedstock composition. Table 66 shows the assumed changes in moisture content between the 2013 SOT and the 2017 Design Case. 
Table 66. Technical targets for biomass field storage of resources in the 2017 Design Case.

\begin{tabular}{lcc} 
Process & \multicolumn{2}{c|}{$\begin{array}{c}\text { After Field Drying } \\
\text { Moisture Content }\end{array}$} \\
\hline Pulpwood & 2013 SOT & 2017 Target \\
\hline $\begin{array}{l}\text { Wood } \\
\text { Residues }\end{array}$ & $40 \%$ & $30 \%$ \\
Switchgrass & $40 \%$ & $30 \%$ \\
\hline
\end{tabular}

\subsubsection{Storage Design Basis}

The 2017 Design Case is based on field drying for pulpwood and forest residues, both to $30 \%$ moisture at the time the material enters landing preprocessing. Field studies on field drying of short rotation southern pine pulpwood and residues have shown final moisture contents of $30 \%$ to be achievable given adequate time ${ }^{655864}$.

Since chips are expected to enter storage at 30\% moisture in the 2017 Design Case, it is reasonable to assume that dry matter losses will be much less (nearly negligible) within the three day holding window. The concerns of unplanned storage extensions, moisture addition, or mechanical losses could increase this number, and therefore the 2017 Design Case assumes a target chip-storage dry matter loss of 5\%. Protection of chip piles with tarps could help to prevent these losses, if the additional material and labor costs are merited, and their presence does not interfere with regular loading and unloading of the piles. Storage of switchgrass is not expected to deviate from the 2013 Design Case baseline. Due to the low moisture content entering storage, the use of a tarp to protect from moisture addition through precipitation has been shown to be sufficient and cost effective when properly applied.

\subsubsection{Biomass Storage Cost Estimation}

Cost estimations for biomass storage were calculated based on literature values from recent reviews ${ }^{74}$, the storage cover vendor's information, and laboratory and field level experiments ${ }^{72}$ (Table 67).

Table 67. Field-side storage cost estimation

\begin{tabular}{|c|c|c|c|c|c|c|}
\hline & \multicolumn{3}{|c|}{ SOT (2011 \$/dry T) } & \multicolumn{3}{|c|}{ Design Target (2011 \$/dry T) } \\
\hline & Storage & Dockage & Total & Storage & Dockage & Total \\
\hline Switchgrass & N/A & N/A & N/A & 5.50 & 0.00 & 5.50 \\
\hline
\end{tabular}

\subsubsection{Preprocessing}

2017 Design Case that incorporates many improvements in preprocessing, including pneumatics, high-moisture densification, and formulation/blending. Figure 42 outlines the material flow given for these improvements. In the 2017 Design Case, substantial cost savings in size reduction are realized by tailoring the preprocessing stages to the individual feedstock and not applying a one size fits all approach. For example, pulpwood is debarked and delimbed and then processed through a chipper to optimize retention of usable material; wood residues are processed through 
a first stage grinder then separated by passing through a trommel screen; switchgrass is processed through a grinder while Construction and Demolition (C\&D) waste undergoes sorting and a wash step.

Separation/sorting of C\&D waste is required to remove recyclables (e.g., metal, paper, and cardboard), contaminants (e.g., plastics and concrete), and other unusable fractions to isolate only those fractions that meet the cost and quality requirements for biofuel feedstocks. In the 2017 Design Case, C\&D is sorted to supply usable material for thermochemical conversion. Prior to any preprocessing the ash content of untreated C\&D waste fractions is estimated to be about $6 \%$. The application of a wash stage further reduces the ash content down to pure wood levels of about $1 \%$.

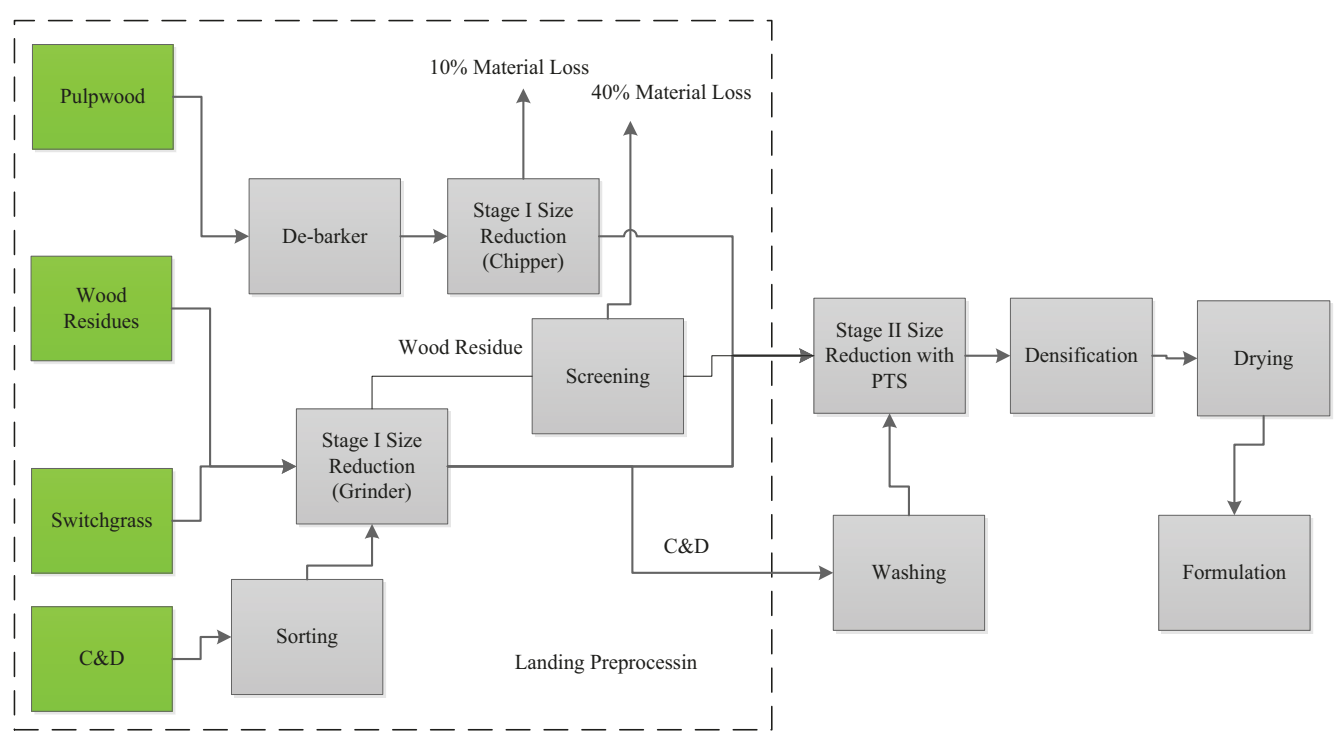

Figure 42. Material flow in the 2017 Design Case that incorporates many improvements in preprocessing, including pneumatics, high-moisture densification, and formulation/blending.

The logistics of a blended feedstock scenario are certainly more complex than a single-feedstock scenario. The 2017 Design Case assumes that preprocessing of C\&D will occur at a preprocessing depot located at the source landfill or refuse transfer station, and C\&D pellets will be shipped from the depot to the blending depot located within proximity of the biorefinery.

Switchgrass that is formatted in large square bales will be delivered to the blending depot, where they will be processed into pellets. Pulpwood and wood residues will be initially processed at the landing for initial size reduction and ash mitigation then transported to a processing facility for pelletization. The pulpwood, wood residues, switchgrass, and C\&D pellets will be queued up in blending bunkers or silos and blended to specification prior to being fed into the conversion process. The pellets of the four blendstocks (i.e., pulpwood, wood residues, switchgrass, and C\&D) are then metered from the blending bunkers in the ratios required of the blended feedstock and are conveyed from the preprocessing facility/depot to the conversion facility. 


\subsubsection{State of Technology:}

For the 2017 Design Case, a geometric mean particle size of 1/4- in. is the target size specification for the thermochemical conversion process design under development by PNNL (Table 68). As the target size specification is the same as biochemical conversion, size reduction system used to meet the final particle size specification required by the end user will be the same for fast pyrolysis conversion. The 2013 state of technology follows sequential two stage size reduction described in section 6

Table 68. Size-reduction design basis

\begin{tabular}{|c|c|c|c|c|}
\hline & & & $2013 \mathrm{SOT}$ & 2017 Target \\
\hline & $\begin{array}{l}\text { Equipment } \\
\text { Used }\end{array}$ & $\begin{array}{l}\text { Screen } \\
\text { Size }\end{array}$ & $\begin{array}{c}\text { Capacity } \\
\text { Ton } / \mathrm{hr}\end{array}$ & $\begin{array}{c}\text { Capacity } \\
\text { Ton } / \mathrm{hr}\end{array}$ \\
\hline \multicolumn{5}{|c|}{ First Stage Size Reduction } \\
\hline Pulpwood & Chipper & $2 \mathrm{in.}$ & 17 & 17 \\
\hline Wood Residue & Grinder & $2 \mathrm{in}$. & & \\
\hline \multicolumn{5}{|l|}{$\begin{array}{l}\text { Second Stage Size } \\
\text { Reduction }\end{array}$} \\
\hline Pulpwood & Hammer mill & $1 / 4$ in. & 5 & 6.5 \\
\hline Wood Residue & Hammer mill & $1 / 4$ in. & 5 & 6.5 \\
\hline
\end{tabular}

\subsubsection{Size Reduction Cost Estimation}

Milling cost estimation is based on vendor-supplied information and equipment performance from typical machine performance and process demonstration unit data and is shown in Table 69. 
Table 69. Size reduction cost estimates.

\begin{tabular}{|lll|}
\hline & $\begin{array}{c}\text { 2013 SOT } \\
(2011 \text { \$dry T })\end{array}$ & $\begin{array}{l}\text { 2017 Target } \\
(2011 \text { \$dry T })\end{array}$ \\
\hline $\begin{array}{l}\text { Total } \\
\text { Pulpwood }\end{array}$ & Total \\
\hline Chipper & 6.10 & 6.10 \\
\hline Debark/delimb & 6.10 & 6.10 \\
\hline Hammer Mill & 17.09 & 13.97 \\
\hline Total & 29.29 & 26.17 \\
\hline Wood Residue & & \\
\hline Grinder & 5.39 & 5.39 \\
\hline Trommel Screen & 3.32 & 3.32 \\
\hline Hammer Mill & 17.09 & 13.97 \\
\hline Total & $\mathbf{2 5 . 8 0}$ & $\mathbf{2 2 . 6 8}$ \\
\hline
\end{tabular}

\subsubsection{Drying and Densification}

A comparison of pellet properties and energy balances for conventional and high-moisture pelletization processes is given in Table 70. The table shows 2017 Design Case targets to achieve a 40 to $50 \%$ reduction in the total pelletization and drying energy.

Table 70. Drying and densification design basis.

\begin{tabular}{|lcc|}
\hline & $2013 \mathrm{SOT}$ & 2017 Target \\
\hline Infeed Moisture & $40 \%$ & $30 \%$ \\
\hline Dryer Moisture Reduction & $28 \%$ & $11 \%$ \\
\hline Densification Moisture Reduction & $3 \%$ & $10 \%$ \\
\hline Final Pellet Moisture & $9 \%$ & $9 \%$ \\
\hline Densification Energy & $75 \mathrm{kWhr} / \mathrm{dry} \mathrm{T}$ & $100 \mathrm{kWhr} / \mathrm{ton}$ \\
\hline Drying Energy & $350 \mathrm{kWhr} / \mathrm{ton}$ & $\mathrm{T}$ \\
\hline Pellet Properties & & $70 \mathrm{lb} / \mathrm{ft}^{3}$ \\
\hline Unit Density & $65 \mathrm{lb} / \mathrm{ft}^{3}$ & $40 \mathrm{lb} / \mathrm{ft}^{3}$ \\
\hline Bulk Density & $35 \mathrm{lb} / \mathrm{ft}^{3}$ & $\mathrm{Greater} \mathrm{than} 97.5 \%$ \\
\hline Durability & $\mathrm{Greater} \mathrm{than} 97.5 \%$ & \\
\hline
\end{tabular}


The high-moisture densification design basis assumptions are as follows:

Our preliminary studies indicated that it is possible to produce high-quality pellets woody material; however, for our 2017 Design Case, we are assuming that the process works for other woody and herbaceous feedstocks to produce durable, high-density pellets.

Technical and cost targets are estimated with the assumption that a grain dryer will be used to dry high-moisture pellets.

Drying of pellets using energy-efficient driers like grain and belt driers is more economical compared to conventional rotary driers.

Slow drying at low temperatures of less than $60^{\circ} \mathrm{C}$ can result in more uniform moisture distribution in pellets.

\subsubsection{Cost Estimation for High-Moisture Densification}

The cost of densification was estimated using vendor-supplied information and the capacity and energy assumptions shown in Table 71.

Table 71. Drying and densification cost estimates.

\begin{tabular}{lcc} 
& $\begin{array}{c}2013 \text { SOT } \\
(2011 \text { \$/dry T) }\end{array}$ & $\begin{array}{c}2017 \text { Target }(2011 \\
\text { \$/dry T) }\end{array}$ \\
\hline Drying & Total & Total \\
Densification & 17.20 & 5.60 \\
Totals & 7.70 & 4.40 \\
\hline
\end{tabular}

\subsubsection{Formulation/Blending}

To meet feedstock specifications required for various conversion pathways, formulation of specific mixtures of feedstocks will likely be required. Examples include mixing high and lowcost feedstocks to meet cost targets, mixing high and low-ash feedstocks to meet an ash target, mixing of high and low-carbohydrate feedstocks to meet a yield target, and mixing easily and poorly reactive feedstocks to meet a convertibility target. An example of blending to meet an ash and moisture specification is shown in Table 72 . 
Table 72. Feedstock formulation/blending of ash and moisture contents*

\begin{tabular}{lccccc|}
$\begin{array}{l}\text { Content Delivered to } \\
\text { Biorefinery Infeed }\end{array}$ & Pulpwood(45\%) & $\begin{array}{c}\text { Wood } \\
\text { Residues(32\%) }\end{array}$ & Switchgrass(3\%) & $\begin{array}{c}\text { C\&D } \\
\text { Waste(20\%) }\end{array}$ & Final Blend \\
\hline $\begin{array}{l}\text { Ash content (wt. \%) } \\
\begin{array}{l}\text { Moisture content (\%, } \\
\text { wet basis) }\end{array}\end{array}$ & 0.5 & 1.0 & 4.0 & 1.0 & $<\mathbf{1 \%}$ \\
HHV (lb/BTU) & 8824 & 9444 & 9 & 9 & $\mathbf{9}$ \\
\hline LHV (lb/BTU) & 7255 & 7616 & 6155 & 8824 & $\mathbf{8 9 8 4}$ \\
\hline
\end{tabular}

*Pulpwood, wood resides, and switchgrass composition data were obtained from the INL Biomass Library ${ }^{75}$.

Assumptions for the formulation design basis are as follows:

- Blended feedstocks are selected and developed to achieve conversion yield specifications. It currently unknown how blended feedstocks will perform in the conversion pathways. The simplest assumption is that the blended feedstocks would be the sum of performances of each individual component. There are on-going trials to test various blended feedstocks and to compare the conversion efficiencies against a single feedstocks.

- Individual feedstocks will be pelleted at depots for shipment to biorefineries. At the biorefinery, these pelleted feedstocks will be unloaded and conveyed into individual bunkers for storage. Pellets of the different blendstocks will be metered out into the bunkers in the ratios required of the blends, crushed (using a pellet crusher), and mixed prior to insertion for the conversion process.

- Material will be metered from individual bunkers onto a conveyer and then thoroughly homogenized through this process with no segregation. Mixing of solids occurs in many industries and is often problematic when solids of varying density, shape, and size are blended. This often leads to segregation, either during the mixing or while being transported to its destination. Mixing of solids is considered a trial-and-error process due to these issues.

- The expected unit operations for formulation are shown in Table 73.

Table 73. Feedstock formulation design basis

\begin{tabular}{lccc|}
\hline 2013 SOT $(2011 \$ /$ dry T) & \multicolumn{2}{c|}{ Operating Parameters } \\
\hline & \multicolumn{1}{c}{ Capacity } & Horsepower \\
\hline $\begin{array}{l}\text { Pellet Pulverizer } \\
\text { Bulk Storage }\end{array}$ & with & 30 ton/hour & $200 \mathrm{HP}$ \\
$\begin{array}{l}\text { Hopper } \\
\text { Conveying System }\end{array}$ & 30 ton/hour & $30 \mathrm{HP}$ \\
\end{tabular}

Research is currently ongoing at INL to examine the compatibility of various feedstocks blends, with an initial focus on the blends reactivity versus the individual feedstocks. Blends will be developed for several regions of the United States using the least-cost formulation model as a 
starting point and will incorporate feedstocks with varying levels of reactivity (e.g., herbaceous, woody, and MSW). Reactivity for the fermentation pathway will be investigated first, with expansion into the other DOE conversion pathways in later fiscal years including bio-oil conversion via fast pyrolysis.

While the costs for preprocessing of feedstocks (e.g., grinding, chemical preconversion, pelleting, and drying) are addressed in other parts of the 2017 Design Case, formulation itself will require a different set of preprocessing options in order to match up with the bio-oil conversion pathway. These processes include bulk storage, conveying systems and a pellet pulverizer to insure that the appropriate recipe of material enters the throat of the conversion reactor in the appropriate blends and sizing requirements.

\subsubsection{Cost Estimation for Formulation.}

Cost estimation for formulation was based on existing technology, vendor-supplied information, and equipment performance (Table 74). These costs are cursory and require more extensive research, especially in their specific application to the bioenergy industry.

Table 74. Formulation cost estimation

\begin{tabular}{|l|c|}
\hline & 2017 Target $(2011 \$ /$ dry T) \\
\hline Pellet pulverizer & 1.10 \\
\hline Bulk storage with hopper & 0.20 \\
\hline Conveying system & 0.60 \\
\hline Totals & 1.90 \\
\hline
\end{tabular}

\subsubsection{Transportation and Handling Design Basis}

The 2017 Design Case includes formulation and densification that meets feedstock specifications and costs targets. Both processes of formulation and densification will improve feedstock handling operations through active controls. Given formulation and the specific quantities of individual feedstocks required, the average transportation distance will change based on feedstock type. In the 2017 Design Case, pulpwood will be trucked from a local draw radius of 50 miles while switchgrass will be trucked fewer than 15 miles. Error! Reference source not

found. in Appendix A show sufficient C\&D waste resources in the selected counties in western South Carolina; therefore, C\&D will be transported by truck from transfer stations after processing. This is not a new concept, transfer stations are already used for sorting and transporting valuable material such as cardboard and scrap metal in densified forms (e.g. baled cardboard, crushed and baled scrap metal). Switchgrass will be loaded and unloaded at each location using a loader (telehandler) capable of moving $12 \mathrm{lb} / \mathrm{ft}^{3}$ bales at $20 \%$ respective moisture content. A 53-ft. trailer and 800,000-GVW limits were assumed in all trucking operations. Transportation for switchgrass will occur from a field side stack to a densification facility completely separate from the conversion facility, but within a minimal conveyor distance (typically $<50$ miles). C\&D waste transportation will occur from the waste transfer station as 
pellets to the preprocessing facility for storage and transfer to the biorefinery. Further transportation and handling assumptions are given as follows:

- At $20 \%$ moisture, transportation continues to be volume limited at densities of $12 \mathrm{lb} / \mathrm{ft}^{3}$.

- At 30\% moisture, transportation of chips continues to be volume limited.

- At $9 \%$ moisture, transportation of pellets is weight limited at $40 \mathrm{lb} / \mathrm{ft}^{3}$.

- There will be insignificant material losses throughout transportation and handling.

- Densification will increase material uniformity and flowability

\subsubsection{Cost Estimation for Transportation and Handling}

The cost estimation for transportation and handling was based on vendor-supplied information and equipment performance from typical machines (Table 75). Rail transportation costs were estimated using a jumbo hopper car ${ }^{54}$ adjusted for U.S. conditions.

Table 75. Transportation cost estimates.

\begin{tabular}{|c|c|c|}
\hline & $\begin{array}{c}2013 \text { SOT } \\
(2011 \$ / \text { dry T) }\end{array}$ & $\begin{array}{l}2017 \text { Target } \\
(2011 \$ / \text { dry T) }\end{array}$ \\
\hline & Total & Total \\
\hline Truck & $14.84^{*}$ & $7.52 * *$ \\
\hline \multicolumn{3}{|c|}{$\begin{array}{l}\text { *Individual feedstock therefore one transportation } \\
\text { pathway }\end{array}$} \\
\hline
\end{tabular}

\subsection{Life Cycle Analysis}

This section uses a life-cycle assessment (LCA) to evaluate the energy input and GHG emissions for conversion In Situ and Ex Situ Upgrading of Fast Pyrolysis Vapors. LCA considered the unit processes involved in the biomass logistics. Table 76 shows the energy consumption and Table 77 show GHG contribution for In Situ and Ex Situ Upgrading of Fast Pyrolysis Vapors Pathway conversion supply chain design. 
Table 76. Energy Consumption for Thermochemical conversion supply chain design.

\begin{tabular}{|c|c|c|c|c|c|}
\hline Process Element & Pulpwood & $\begin{array}{l}\text { Wood } \\
\text { Residues }\end{array}$ & Switchgrass & $\begin{array}{l}\text { Construction and } \\
\text { Demolition Waste } \\
\text { (C\&D) }\end{array}$ & Blend \\
\hline Formulation Contribution & $45 \%$ & $32 \%$ & $3 \%$ & $20 \%$ & - \\
\hline $\begin{array}{l}\text { Harvest and Collection } \\
(\text { MBTU/dry T) }\end{array}$ & 182.78 & - & 122.85 & - & 85.94 \\
\hline $\begin{array}{l}\text { Landing Preprocessing/ } \\
\text { Sorting (MBTU/dry T) }\end{array}$ & 231.52 & 110.25 & - & 410.25 & 221.51 \\
\hline $\begin{array}{l}\text { Transportation } \\
\text { (MBTU/dry T) }\end{array}$ & 136.87 & 101.38 & 99.35 & 273.73 & 151.76 \\
\hline $\begin{array}{l}\text { Preprocessing (MBTU/dry } \\
\text { T) }\end{array}$ & 408.01 & 408.01 & 285.83 & 408.01 & 404.34 \\
\hline Storage (MBTU/dry T) & 8.46 & 8.46 & 21.83 & 8.46 & 8.86 \\
\hline Handling (MBTU/dry T) & 42.69 & 42.69 & 41.90 & 42.69 & 42.67 \\
\hline $\begin{array}{l}\text { Total Energy Consumption } \\
\text { (MBTU/dry T) }\end{array}$ & 1001.33 & 670.79 & 571.76 & 1143.14 & 915.08 \\
\hline
\end{tabular}

Table 77. GHG contribution for thermochemical conversion supply chain design.

\begin{tabular}{|c|c|}
\hline Process Element & Blend \\
\hline Formulation Contribution & - \\
\hline $\begin{array}{l}\text { Harvest and collection GHGs ( } \mathrm{Kg} \mathrm{CO}_{2} \mathrm{e} / \text { dry } \\
\mathrm{T})\end{array}$ & 8.65 \\
\hline $\begin{array}{l}\text { Landing Preprocessing GHGs }\left(\mathrm{Kg} \mathrm{CO}_{2} \mathrm{e} / \text { dry }\right. \\
\mathrm{T})\end{array}$ & 18.95 \\
\hline Transportation GHGs $\left(\mathrm{Kg} \mathrm{CO}_{2} \mathrm{e} /\right.$ dry $\left.\mathrm{T}\right)$ & 8.62 \\
\hline Preprocessing GHGs $\left(\mathrm{Kg} \mathrm{CO}_{2} \mathrm{e} / \mathrm{dry} \mathrm{T}\right)$ & 34.60 \\
\hline Storage GHGs $\left(\mathrm{Kg} \mathrm{CO}_{2} \mathrm{e} / \mathrm{dry} \mathrm{T}\right)$ & 0.89 \\
\hline Handling GHGs ( $\left.\mathrm{Kg} \mathrm{CO}_{2} \mathrm{e} / \mathrm{dry} \mathrm{T}\right)$ & 4.2 \\
\hline Total GHGs (Kg CO $2 \mathrm{e} / \operatorname{dry} \mathrm{T})$ & 75.91 \\
\hline
\end{tabular}




\section{Dilute-Acid Conversion of Lignocellulosic Biomass to High Octane Gasoline via Indirect Gasification and Methanol Intermediate}

This sections is intended to couple with the National Renewable Energy Laboratory's (NREL's) hydrocarbon design report, "Process Design and Economics for the Conversion of Lignocellulosic Biomass to High Octane Gasoline via Indirect Gasification and Methanol Intermediate" that describes a viable route from biomass to hydrocarbon fuels. The assumptions of scale and feedstock quality requirements are consistent with the design case assumptions used by NREL in their report and techno economic assessments. This design does not consider the different requirements and nuances of other thermochemical conversion processes or other hydrocarbon pathways.

\subsection{Summary}

This report establishes a plausible case for achieving the 2017 Design Case for Lignocellulosic Biomass to High Octane Gasoline via Indirect Gasification and Methanol Intermediate conversion cost goals of delivering a biomass feedstock to the conversion facility at a cost of $\$ 80 /$ dry T (Table 78). The least-cost formulation approach (Appendix B) illustrates the importance of cost estimates for determining the total cost of feedstock to a biorefinery, including grower payment (access costs), logistics costs, and quality/dockage cost. It also illustrates the importance of refining and updating these costs as analyses and data improve to better inform the estimates. The following conclusions are presented to document the specific areas that require additional attention to further strengthen and support the feedstock design detailed in this report. 
Table 78. Thermochemical feedstock design cost analysis for 2017.

\begin{tabular}{|c|c|c|c|c|c|}
\hline Cost Element & Pulpwood & Wood Residues & Switchgrass & $\begin{array}{l}\text { Construction and } \\
\text { Demolition Waste } \\
\text { (C\&D) }\end{array}$ & Blend \\
\hline $\begin{array}{l}\text { Formulation } \\
\text { Contribution }\end{array}$ & $45 \%$ & $32 \%$ & $3 \%$ & $20 \%$ & - \\
\hline $\begin{array}{l}\text { Grower payment/ } \\
\text { access cost }\end{array}$ & 25.00 & 26.35 & 19.67 & 8.15 & 21.90 \\
\hline $\begin{array}{l}\text { Harvest and } \\
\text { collection }(\$ / \text { dry } T)\end{array}$ & 22.24 & 0 & 15.41 & - & 10.47 \\
\hline $\begin{array}{l}\text { Landing } \\
\text { Preprocessing/ } \\
\text { Sorting (\$dry T) }\end{array}$ & 12.17 & 8.73 & 0 & 9.85 & 10.24 \\
\hline $\begin{array}{l}\text { Transportation } \\
\text { (\$/dry T) }\end{array}$ & 10.89 & 3.33 & 4.5 & 6.87 & 7.47 \\
\hline $\begin{array}{l}\text { Preprocessing } \\
(\$ / \text { dry T) }\end{array}$ & 23.97 & 23.97 & 19.7 & 28.12 & 24.67 \\
\hline Storage (\$/dry T) & 3.23 & 3.23 & 5.5 & 3.23 & 3.30 \\
\hline Handling (\$/dry T) & 1.90 & 1.90 & 1.90 & 1.90 & 1.90 \\
\hline $\begin{array}{l}\text { Total Delivered } \\
\text { Feedstock Cost } \\
\text { (\$/dry T) }\end{array}$ & 99.49 & 67.51 & 66.68 & 58.12 & 80.00 \\
\hline \multicolumn{6}{|c|}{ Delivered Feedstock Specifications } \\
\hline Ash content (wt. \%) & 0.5 & 1.0 & 4.0 & 1.0 & $<1 \%$ \\
\hline $\begin{array}{l}\text { Moisture content } \\
\text { (\%, wet basis) }\end{array}$ & 9 & 9 & 9 & 9 & 9 \\
\hline HHV (BTU/lb) & 8824 & 9444 & 7557 & 8824 & 8984 \\
\hline LHV (BTU/lb) & 7255 & 7616 & 6155 & 7255 & 7337 \\
\hline
\end{tabular}

Continued refinements of the biomass supply curves to represent the latest estimates for biomass grower payment are needed to support the least-cost formulation approach. Ultimately, translating The Billion Ton Update ${ }^{5}$ data from farm gate price to grower payment is necessary to establish better grower payment estimates. The grower payment estimates included in this report were calculated by subtracting our harvest and collection costs from the farm gate price.

Logistics costs are based on actual field trial data but do not include the cost of various business elements, such as profit margins for transportation, depots and field agents that would be involved throughout a biomass feedstock supply chain. This would increase the overall cost of the supply system than is demonstrated in this report. This was of little consequence to the 2012 Conventional Design Case target that intentionally focused only on logistics costs. The 2017 Design Case, on the other hand, is meant to encompass total delivered feedstocks costs. Further, the complexity of a blended feedstock approach may introduce multiple business elements into 
the supply chain; therefore, it is important that logistics costs be updated to include the true cost of these business elements, including a return on investment.

As the biomass logistic systems become more complex, especially with the introduction of new technologies (e.g., chemical preconversion), it may be prudent to differentiate between the current state-of-technology costs and the projected costs of mature technology ( ${ }^{\text {th }}$ plant costs) to be consistent with conversion platform terminology. This was not an issue with conventional feedstock designs that were intrinsically tied to current SOT; however, for technology maturation, cost reductions may be worth considering for advanced feedstock designs.

Admittedly, it also is necessary to tighten the design and cost estimates around formulation and the engineering systems for crushing the pellets and blending prior to insertion into the conversion process. A better understanding of C\&D availability, cost, and conversion performance is needed to solidify its position in the 2017 Design Case. Likewise, the viability of blended feedstocks as a whole depends on their conversion performance. DOE Bioenergy Technology Office funded research is investigating the conversion performance of blends (including C\&D blends) and evaluating the compatibilities and incompatibilities of blendstocks. The results of this research are critical to further development of blended feedstocks.

\subsection{Feedstock Composition (In-feed quality specifications)}

The dry basis elemental composition of the feedstock, shown in Table 79 is similar to previous NREL and PNNL design reports ${ }^{6}$. The composition was originally assumed to come from pulpwood. Recent feedstock logistics work suggests the use of blended material may be required to meet a cost target of $\$ 80 /$ dry US ton while still meeting these specifications. For the purpose of this report, it is assumed that any blended material provided to meet this feedstock elemental composition will not adversely affect fast pyrolysis conversion efficiencies. Ongoing studies being conducted jointly by INL, NREL and PNNL will provide experimental evidence of the impact of blended feedstocks on fast pyrolysis and gasification processes. Future TEA will be modified to reflect conversion impacts inferred from such studies. As has been described in prior conversion design reports, the feedstock composition plays a critical role on overall process design and economics, primarily with respect to high and low heating values, carbon as well as high sensitivity to impurity components such as ash and metals in this design. The blended uniform-format feedstock composition assumed here for purposes of future design case targets is shown below, with supporting details described in the 2011 ethanol report ${ }^{46}$. Also consistent with prior design cases, the moisture content for the delivered feedstock is $10 \%$ or less. 
Table 79. Delivered woody feedstock composition and processing assumptions for the fast pyrolysis and hydrotreating design report ${ }^{6}$.

\begin{tabular}{|lc|}
\hline Component & $\begin{array}{c}\text { Composition } \\
\text { (dry wt. \%) }\end{array}$ \\
\hline Carbon & 50.94 \\
\hline Hydrogen & 6.04 \\
\hline Nitrogen & 0.17 \\
Sulfur & 0.03 \\
\hline Oxygen & 41.90 \\
\hline Ash & $0.90-1.0$ \\
\hline Heating Value (Btu/lb) & $8,601 \mathrm{HHV}$ \\
\hline Moisture (Bulk Wt. \%) & $7,996 \mathrm{LHV}$ \\
\hline Particle Size (inch) & 10.0 \\
\hline
\end{tabular}

\subsection{Feedstock Selection Cost Estimation}

Expansion beyond highly productive resource areas has significant implications to the feedstock supply chain. Sparse areas, whether due to reduced yields and/or higher dispersion, typically increase feedstock logistics costs. Higher harvest and collection costs are incurred due to the need to spread machinery ownership costs over fewer tons of biomass or the need to cover more acres for the same quantity of biomass. Additionally, lower resource yields increase the supply radius and biomass transportation distances. Under the 2012 Conventional Design, higher yield areas allow refinery to be selective on the resource that they access.

Consider, for example, the scenarios depicted in Figure 43. This resource map illustrates a county-level resource assessment of pulpwood farm gate at $\$ 60 /$ dry T prices (this includes grower payment, harvest, collection, and chipping costs). Farm gate price data were extracted from The Billion Ton Update (BT2) ${ }^{5}$ data supplied from Oak Ridge National Laboratory. It should be noted that while the data is reported at a county-level, the data should be applied at the wood shed (typically much larger area than a county) level because it was derived from the U.S. Forest Service Forest Inventory and Assessment Data (FIA) ${ }^{56}$ and does not equate to county levels accurately. The FIA is a woodshed level assessment and therefore to use the data correctly it is necessary to combine multiple counties.

The cost competitiveness of the 2012 Conventional Design was demonstrated by in the scenario located in southern Alabama, a high biomass yielding area ${ }^{3}$. We further suggest, based on the consistency of farm gate (i.e., landing) prices shown in this map, that the 2012 Conventional Design can be deployed cost effectively in South Carolina. Commercial readiness of conventional supply systems ultimately will be demonstrated by commercial-scale cellulosic ethanol plants opening in these areas in the near future. 


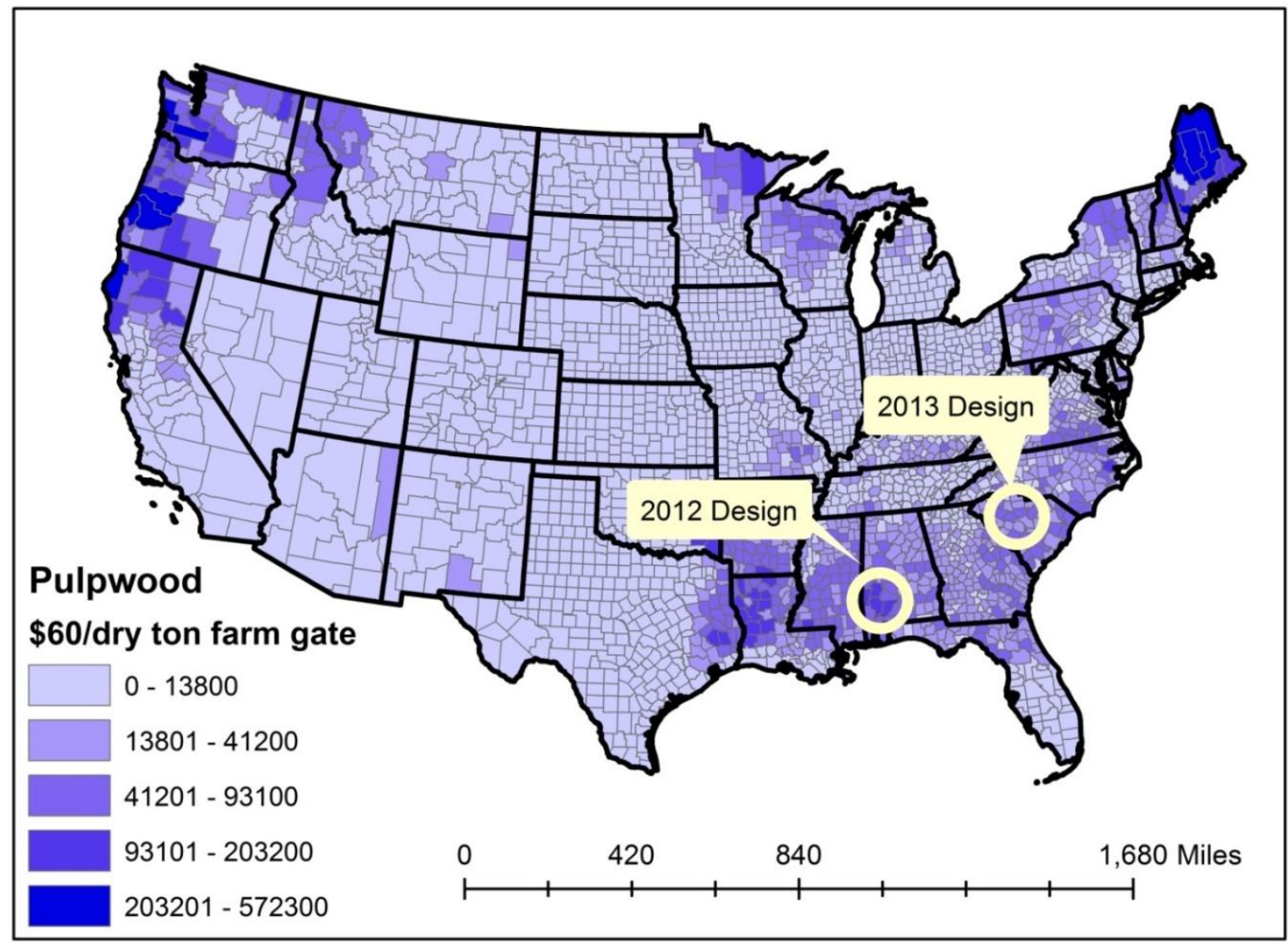

Figure 43. Total tons per county of available pulpwood at $\$ 60 /$ dry $\mathrm{T}$ farm gate price. Yellow circles show areas represented in the 2012 Conventional Design and the Relocated (2013) Design Case ${ }^{5}$.

Access costs are calculated from the grower payment cost curves shown in Figure 6, which are derived from historical prices. The 2017 Design Case basis discussion presented above provided the least-cost formulation approach for reducing access costs by accessing multiple feedstocks. With this approach, reduced quantities of each feedstock allows us to stay lower on the supply curve than if we had to supply the entire refinery with any single feedstock. The impact of this approach is shown in Table 80. The 2013 State of Technology assumes a 100\% supply of pulpwood of 909,100 dry $\mathrm{T}$ at an estimated $\$ 60 /$ dry $\mathrm{T}$ farm gate or a $\$ 25 /$ dry $\mathrm{T}$ access cost. In comparison, the 2017 Design Case blend of 45\% pulpwood, 32\% wood residues, $20 \% \mathrm{C} \& \mathrm{D}$ waste, and 3\% switchgrass results in a weighted average feedstock cost that is nearly $15 \%$ lower than the access cost of pulpwood alone. 
Table 80. Resource access cost estimate (U.S. DOE $2011^{5}$, and INL MSW Data).

\begin{tabular}{lcccc} 
& \multicolumn{2}{c}{ 2013 SOT } & \multicolumn{2}{c}{ 2017 Target } \\
& $\begin{array}{c}\text { Access Cost } \\
(2011 \text { \$dry T) }\end{array}$ & Tons & $\begin{array}{c}\text { Access Cost } \\
(2011 \text { \$dry T) }\end{array}$ & Tons \\
Pulpwood & 25.00 & $909,100^{*}$ & 25.00 & $425,700^{*}$ \\
Wood Residues & NA & NA & 26.35 & $412,800^{* *}$ \\
Switchgrass & NA & NA & 19.67 & 25,800 \\
C\&D & NA & NA & 8.15 & 172,000 \\
Totals & 25.00 & NA & 21.90 & $1,036,300$ \\
& *assumes & $10 \%$ loss of material to debark/delimb (Walker, 2006) &
\end{tabular}

\subsection{Quality Specification and Design Assumptions}

The 2012 Conventional Design focused on conventional woody harvest operations (i.e., felling and/ or skidding operations are separate from landing preprocessing operations). In addition to including switchgrass into the feedstock blend, the 2017 Design Case assumes that the immaturity of the biomass market will limit the forest and farm owner's investment in advanced equipment options for both woody and herbaceous feedstocks. Therefore, with the exception of a few proactive, early adopters, conventional forestry and farming operations will dominate the market in the regions defined by the 2017 Design Case. Table 81 below summarizes the assumptions and differences between the 2012 and 2017 logistic designs. 
Table 81. Summary of assumptions underpinning progressive design implementations ${ }^{57}$.

\begin{tabular}{|c|c|c|c|}
\hline & $\begin{array}{c}2012 \\
\text { Conventional } \\
\text { Design }\end{array}$ & Baseline & 2017 Design Case \\
\hline Feedstock(s) & Pulpwood & Pulpwood & $\begin{array}{l}\text { Blended feedstock: pulpwood }(45 \%) \text {, wood } \\
\text { residues }(32 \%) \text {, switchgrass }(3 \%) \text {, and select } \\
\text { construction and demolition wastes (C } \\
\& D)(20 \%)\end{array}$ \\
\hline $\begin{array}{l}\text { Grower } \\
\text { payment }\end{array}$ & $\begin{array}{l}\text { Breakeven cost of } \\
\text { production }\end{array}$ & $\begin{array}{l}\text { Increases based } \\
\text { on marginal } \\
\text { cost differential }\end{array}$ & $\begin{array}{l}\text { Calculated and modeled according to specific } \\
\text { location and resource blend/formulation }\end{array}$ \\
\hline Moisture & Field dried to $40 \%$ & $\begin{array}{l}\text { Field dried to } \\
40 \%\end{array}$ & $\begin{array}{l}\text { Arrives: Pulpwood chips } 30 \% \text { wood residue } \\
\text { chips } 30 \% \text {, switchgrass } 20 \% \text {, and C\& D } \\
\text { ground } 20 \% \text {; } \\
\text { All dried to } 9 \% \text { pellets }\end{array}$ \\
\hline Ash & Debark/delimb & Debark/delimb & $\begin{array}{l}\text { Debark/delimb pulpwood } \\
\text { Trommel screen residues } \\
\text { Wash and sort C\& D waste } \\
\text { Blended ash content of }<1 \% \\
\text { Debarked pulpwood }<1 \% \text {, screened wood } \\
\text { residues } 1.4 \% \text {; washed and sorted C\&D } 1.0 \%\end{array}$ \\
\hline Logistics & $\begin{array}{l}\text { Uses existing } \\
\text { systems }\end{array}$ & $\begin{array}{l}\text { Uses existing } \\
\text { systems }\end{array}$ & $\begin{array}{l}\text { Pneumatics attached to hammermill } \\
\text { High-moisture densification }\end{array}$ \\
\hline $\begin{array}{l}\text { Quality } \\
\text { controls } \\
\text { (passive) }\end{array}$ & $\begin{array}{l}\text { Field drying to } \\
\text { reduce moisture } \\
\text { Ample available } \\
\text { resource; quality } \\
\text { spec manually } \\
\text { selected }\end{array}$ & $\begin{array}{l}\text { Field drying to } \\
\text { meet moisture } \\
\text { spec }\end{array}$ & $\begin{array}{l}\text { Harvest/collection and storage best management } \\
\text { practices for pulpwood and switchgrass } \\
\text { More rigorous field drying of pulpwood and } \\
\text { residues }\end{array}$ \\
\hline $\begin{array}{l}\text { Quality } \\
\text { controls } \\
\text { (active) }\end{array}$ & Waste heat dryer & Rotary drying & $\begin{array}{l}\text { Multiple resource blending/formulation } \\
\text { High-moisture densification } \\
\text { High-efficiency pellet drying }\end{array}$ \\
\hline $\begin{array}{l}\text { Meets quality } \\
\text { target }\end{array}$ & Yes & Yes & Yes \\
\hline $\begin{array}{l}\text { Meets cost } \\
\text { target }\end{array}$ & Yes & No & Yes \\
\hline $\begin{array}{l}\text { Accesses } \\
\text { dispersed } \\
\text { resources }\end{array}$ & No & No & Yes \\
\hline
\end{tabular}


Relative to the woody feedstocks used in the Thermochemical Design Case, the 2013 baseline and 2017 Design Case are similar in many ways for harvest and collection, but the latter has two key changes to improve quality and production of woody materials. While each system is discussed later, the key differences of the 2017 Design Case are first inclusion of woody residues sourced from pulpwood operations, and second in-forest drying of whole tree piles at the landing to achieve a more aggressive moisture content of 30\%.In this design debarking and delimbing are conducted to improve biomass quality. Construction and demolition wastes are considered to enter the feedstock logistics system at the preprocessing stage and are therefore not discussed here.

\subsection{Feedstock Logistics}

\subsubsection{Harvest and Collection}

Conventional wood harvest and collection relies on existing forestry technologies designed for timber and pulp and paper production. Collection systems for woody material involve cutting the feedstock with a tracked feller buncher and transporting the material to the landing with a grapple skidder immediately after felling. Felling and skidding operations increase the overall ash content of harvested whole-trees by introducing soil as it is moved in contact with the ground from one location to the next ${ }^{58}$. Thermochemical conversion processes via fast pyrolysis are highly sensitive to silica, alkali, and transition metals found in most soils, impacting process performance $^{596061}$. In both the 2013 and 2017 Design Cases active management strategies are employed during the landing preprocessing to improve biomass ash content .Current forestry production of pine pulpwood is reported to yield roundwood with a moisture content of 45-55\% and a whole-tree ash content ranging from 1\% to 3\% ${ }^{62} 6364$. The 2013 baseline assumes the moisture content of pulpwood to be $50 \%$ for whole-trees entering storage.

Woody residues are generated through typical commercial forestry operations on southern pine plantations where trees are harvested for pulpwood, chip-and-saw, and saw timber. Similar to the above described collection of pulpwood, these operations bring whole trees to the landing where they are delimbed and topped using a pull-through delimber. The roundwood is then loaded onto trucks for delivery to the mill while the residues are piled at the landing. While not collected in the 2013 baseline, the 2017 Design Case utilizes these materials as a fraction of the feedstock blend. The baseline for residue moisture content is reported at $40 \%$, while ash content has been reported to range from $2 \%$ to $4 \% 47,62,6566$. Switchgrass, which is part of the blend, harvest and collection systems use conventional windrowing harvester and rectangular baler $(3 \mathrm{x} 4 \mathrm{x} 8 \mathrm{-ft})$.

\subsubsection{Harvest and Collection Design Basis}

The 2017 Design Case incorporates a chain flail debarker during preprocessing at the landing to increase the quality of the final chipped pulpwood product. Therefore, moisture and ash contents of material entering storage are not different between the 2013 and 2017 Designs. However, there is still impetus to increase the operational efficiency of roundwood collection for reducing costs ${ }^{6768}$. This can be achieved through forest management shifts to short-rotation pine plantations aimed at supplying bioenergy production, increased efficiency of harvesting machinery, and increased efficiency of grapple skidder transportation. Research conducted by Auburn University for the DOE High Tonnage Forest Biomass Project has demonstrated high capacity grapplers to increase productivity by 80 tons per productive machine hour compared to 
traditional systems ${ }^{58}$. Figure 44 depicts a conventional skidder and a high capacity skidder. Further development of such operational improvements will play a key role in reducing costs of clean pulp chips for thermochemical conversion. In addition, transition of forest management to short rotation pine plantations focused on energy use is a promising option for increasing yields; should the economics of establishment be overcome ${ }^{69}$.

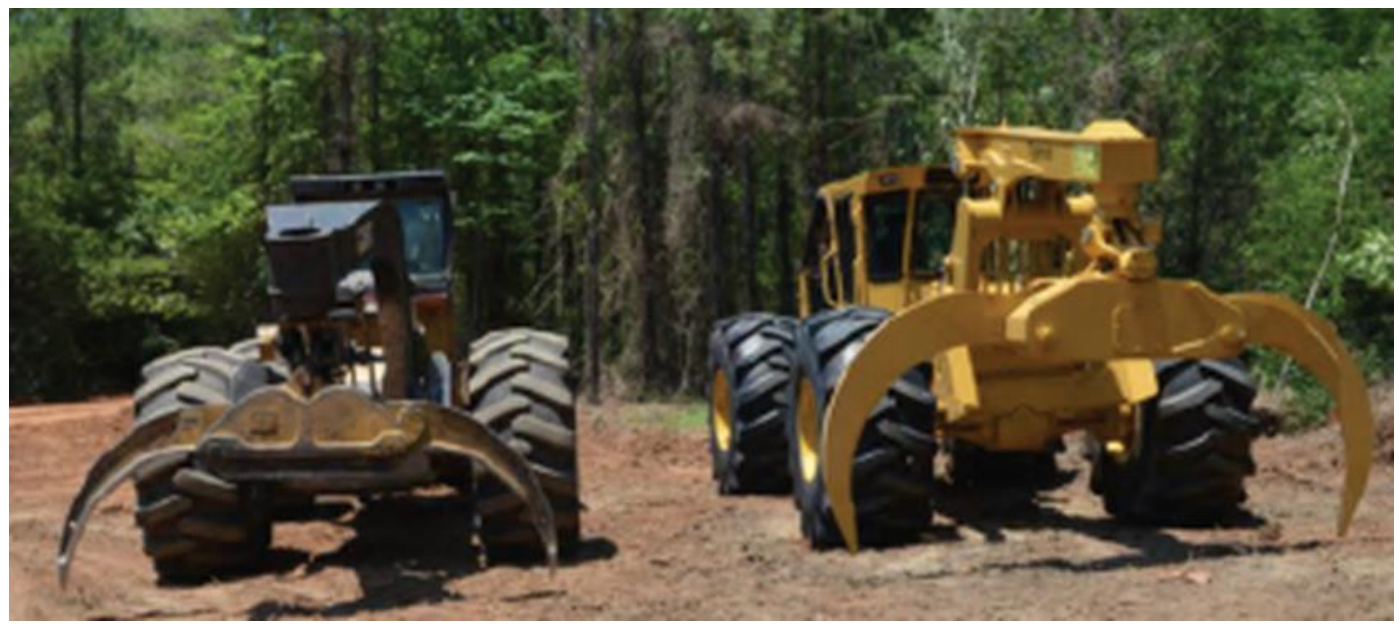

Figure 44. Conventional (left) and high-capacity grapple skidder (right) for transporting small diameter pulpwood from the forest to the landing. Photo credit: Auburn University High Tonnage Forest Biomass Project ${ }^{58}$.

Wood residues (tree tops and limbs) originate from other commercial logging operations and are located in piles at the landing, eliminating the costs for harvest and collection (e.g., felling and skidding). Similar to pulpwood, the 2017 Design Case incorporates active quality controls to reduce the ash content during preprocessing at the landing. These active controls applied after storage may contain ash contents in excess of the desired specification of $0.9 \%$ for wood residues and less for pulpwood.

Switchgrass harvest in the 2017 Design Case follows conventional practices for feed and forage in terms of the equipment used, but incorporates more rigorous passive quality controls to reduce ash content. Delayed harvest of switchgrass provides the benefits of reducing moisture and ash content, but even with the practice of delayed-harvest, it is clear the raw feedstock will not meet the final quality specification for ash. Blending of switchgrass with a low-ash feedstock is necessary to achieve ash specification of $<1 \%$. Nevertheless, it is important that best management practices for switchgrass harvest are used to reduce soil contamination during the processes of cutting and baling while respecting the relationship between delayed harvest date and collection efficiency. Research conducted by Oklahoma State University in collaboration with INL shows that switchgrass can achieve moisture contents at or below the 2017 Design Case specification ( $10 \%$ to $5 \%$ ), though climatic variance can still introduce moisture variability in delayed harvests (Figure 45). In this same research the ash content of switchgrass was found to be low even at an early harvest (5\% in August), though a decreasing trend was observed as harvest was delayed ( $4 \%$ by December). This work stands as an example of the effectiveness of proper harvesting techniques, and stresses the importance of establishing best management 
practices to cope with variability in weather conditions. Goals for the 2017 Design Case include reducing ash content to $4 \%$ through harvest timing and advanced harvesting techniques.

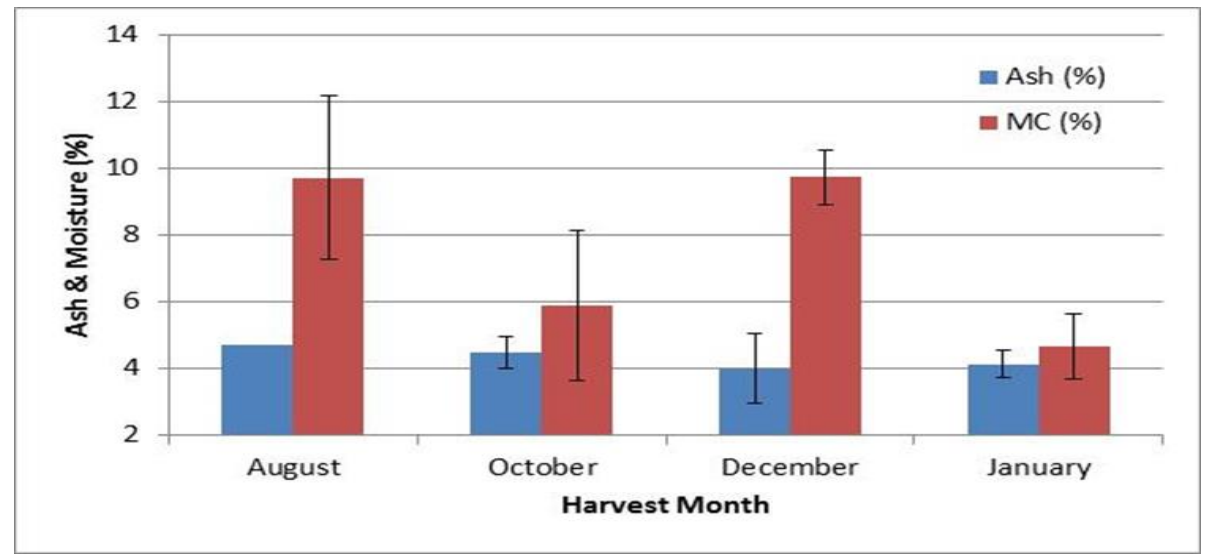

Figure 45. Ash and moisture content of switchgrass harvested in Oklahoma, 2010 by Oklahoma State University. Error bars represent one standard deviation. Ash samples for October, December, and January are three samples comprised of six individual core samples composited.

\subsubsection{Harvest and Collection Cost Estimation}

Harvest and collection costs assume a removal rate of 15 dry T/acre for pulpwood ${ }^{70}, 4$ dry T/acre for residues ${ }^{66}$, and 5-dry T/acre for switchgrass ${ }^{71}$. These assumptions are consistent with those used in the Billion Ton Update ${ }^{5}$. Cost of harvest and collection is shown in Table 82 . The cost of ash at this point within the feedstock logistics system described in section 4 is not yet applicable to pulpwood or wood residue, as the material will undergo active quality controls during landing preprocessing. Switchgrass may be subjected to an ash dockage at this point in the process if ash contents are greater than those needed by the feedstock blending process. 
Table 82. Biomass harvest and collection cost estimates derived from INL analysis.

\begin{tabular}{|c|c|c|}
\hline \multirow[t]{2}{*}{ Machine } & $\begin{array}{c}2013 \text { SOT } \\
(2011 \text { \$/dry T) }\end{array}$ & $\begin{array}{c}2017 \text { Target (2011 } \\
\text { \$/dry T) }\end{array}$ \\
\hline & Total & Total \\
\hline \multicolumn{3}{|l|}{ Pulpwood } \\
\hline Felling & 15.00 & 15.00 \\
\hline Yarding & 7.24 & 7.24 \\
\hline Totals & 22.24 & 22.24 \\
\hline \multicolumn{3}{|l|}{ Wood Residues* } \\
\hline Felling & 0.00 & 0.00 \\
\hline Yarding & 0.00 & 0.00 \\
\hline Totals & 0.00 & 0.00 \\
\hline \multicolumn{3}{|l|}{ Switchgrass } \\
\hline Mower-conditioner & 4.80 & 4.80 \\
\hline Baler & 7.30 & 7.30 \\
\hline $\begin{array}{l}\text { Bale } \\
\text { collection/stacking }\end{array}$ & 3.30 & 3.30 \\
\hline Totals & 15.40 & 15.40 \\
\hline
\end{tabular}

\subsubsection{Storage}

\subsubsection{2013 State of Technology}

Because the 2017 Design Case utilizes a blended feedstock, switchgrass storage must be addressed. The storage of switchgrass occurs field side or at a similar on-farm unimproved storage site. As for any baled feedstock, appropriate storage sites provide adequate drainage away from the stack to prevent the accumulation of moisture around the stack, provide yearround access, and preferably allow stack to be positioned in a North-South orientation to reduce moisture accumulation on the north side of the stack ${ }^{72}$. Tarped stacks are chosen as a balance between bale protection against moisture infiltration, which leads to dry matter loss, and storage configuration costs ${ }^{73} 22$. Stacks are constructed with a self-propelled stacking bale wagon and are six bales high and covered with a high-quality hay tarp. To prolong tarp life, it is also important that adequate year-round maintenance be provided to periodically tighten the tarps ${ }^{74}$. Biomass storage systems in the current Design Case seek to provide a low-cost, lowmaintenance, moisture-tolerant solution that focus on maintaining moisture content $<20 \%$, minimizing dry matter loss and preserving feedstock composition. Table 83 shows the assumed changes in moisture content between the 2013 SOT and the 2017 Design Case. 
Table 83. Technical targets for biomass field storage of resources in the 2017 Design Case.

\begin{tabular}{|lcr|}
\hline Process & \multicolumn{2}{c|}{$\begin{array}{c}\text { After Field Drying } \\
\text { Moisture Content }\end{array}$} \\
\hline Pulpwood & 2013 SOT & 2017 Target \\
Wood & $40 \%$ & $30 \%$ \\
Residues & $40 \%$ & $30 \%$ \\
Switchgrass & $20 \%$ & $20 \%$
\end{tabular}

\subsubsection{Storage Design Basis}

The 2017 Design Case is based on field drying for pulpwood and forest residues, both to $30 \%$ moisture at the time the material enters landing preprocessing. Field studies on field drying of short rotation southern pine pulpwood and residues have shown final moisture contents of $30 \%$ to be achievable given adequate time ${ }^{655864}$.

Since chips are expected to enter storage at 30\% moisture in the 2017 Design Case, it is reasonable to assume that dry matter losses will be much less (nearly negligible) within the three day holding window. The concerns of unplanned storage extensions, moisture addition, or mechanical losses could increase this number, and therefore the 2017 Design Case assumes a target chip-storage dry matter loss of 5\%. Protection of chip piles with tarps could help to prevent these losses, if the additional material and labor costs are merited, and their presence does not interfere with regular loading and unloading of the piles. Storage of switchgrass is not expected to deviate from the 2013 Design Case baseline. Due to the low moisture content entering storage, the use of a tarp to protect from moisture addition through precipitation has been shown to be sufficient and cost effective when properly applied.

\subsubsection{Biomass Storage Cost Estimation}

Cost estimations for biomass storage were calculated based on literature values from recent reviews ${ }^{74}$, the storage cover vendor's information, and laboratory and field level experiments ${ }^{72}$ (Table 84).

Table 84. Field-side storage cost estimation.

\begin{tabular}{lllllll} 
& & SOT $(2011 \$ /$ dry T $)$ & \multicolumn{3}{c}{ Design } & Target $(2011$ \$/dry T) \\
\hline \multirow{2}{*}{ Switchgrass } & Storage & Dockage & Total & Storage & Dockage & Total \\
& N/A & N/A & N/A & 5.50 & 0.00 & 5.50
\end{tabular}

\subsubsection{Preprocessing}

The 2017 Design Case that incorporates many improvements in preprocessing, including pneumatics, high-moisture densification, and formulation/blending. Figure 46 outlines the material flow given for these improvements. In the 2017 Design Case, substantial cost savings in size reduction are realized by tailoring the preprocessing stages to the individual feedstock and not applying a one size fits all approach. For example, pulpwood is debarked and delimbed and then processed through a chipper to optimize retention of usable material; wood residues are 
processed through a first stage grinder then separated by passing through a trommel screen; switchgrass is processed through a grinder while Construction and Demolition (C\&D) waste undergoes sorting and a wash step.

Separation/sorting of C\&D waste is required to remove recyclables (e.g., metal, paper, and cardboard), contaminants (e.g., plastics and concrete), and other unusable fractions to isolate only those fractions that meet the cost and quality requirements for biofuel feedstocks. In the 2017 Design Case, C\&D is sorted to supply usable material for thermochemical conversion. Prior to any preprocessing the ash content of untreated C\&D waste fractions is estimated to be about $6 \%$. The application of a wash stage further reduces the ash content down to pure wood levels of about $1 \%$.

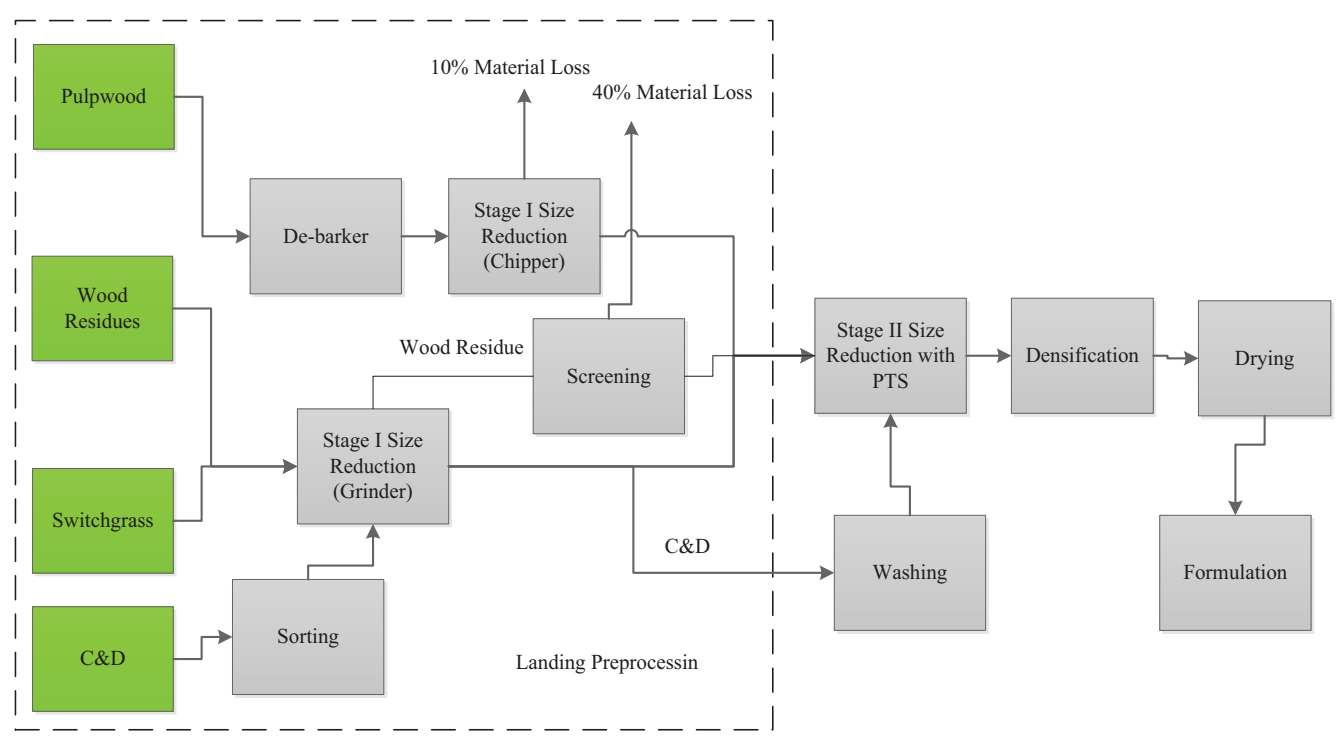

Figure 46. Material flow in the 2017 Design Case that incorporates many improvements in preprocessing, including pneumatics, high-moisture densification, and formulation/blending.

The logistics of a blended feedstock scenario are certainly more complex than a single-feedstock scenario. The 2017 Design Case assumes that preprocessing of C\&D will occur at a preprocessing depot located at the source landfill or refuse transfer station, and C\&D pellets will be shipped from the depot to the blending depot located within proximity of the biorefinery.

Switchgrass that is formatted in large square bales will be delivered to the blending depot, where they will be processed into pellets. Pulpwood and wood residues will be initially processed at the landing for initial size reduction and ash mitigation then transported to a processing facility for pelletization. The pulpwood, wood residues, switchgrass, and C\&D pellets will be queued up in blending bunkers or silos and blended to specification prior to being fed into the conversion process. The pellets of the four blendstocks (i.e., pulpwood, wood residues, switchgrass, and C\&D) are then metered from the blending bunkers in the ratios required of the blended feedstock and are conveyed from the preprocessing facility/depot to the conversion facility. 


\subsubsection{State of Technology:}

For the 2017 Design Case, a geometric mean particle size of 1/4- in. is the target size specification for the thermochemical conversion process design under development by PNNL (Table 85). As the target size specification is the same as biochemical conversion, size reduction system to meet the final particle size specification required by the end user will be the same for fast pyrolysis conversion. The 2013 state of technology follows sequential two stage size reduction described in section 9.

Table 85. Size-reduction design basis.

\begin{tabular}{|c|c|c|c|c|}
\hline & & & $\begin{array}{l}2013 \\
\text { SOT }\end{array}$ & 2017 Target \\
\hline & $\begin{array}{l}\text { Equipment } \\
\text { Used }\end{array}$ & $\begin{array}{l}\text { Screen } \\
\text { Size }\end{array}$ & $\begin{array}{c}\text { Capacity } \\
\text { Ton } / \mathrm{hr}\end{array}$ & $\begin{array}{c}\text { Capacity } \\
\text { Ton } / \mathbf{h r}\end{array}$ \\
\hline \multicolumn{5}{|c|}{ First stage Size Reduction } \\
\hline Pulpwood & Chipper & $2 \mathrm{in.}$ & 17 & 17 \\
\hline Wood Residue & Grinder & 2in. & & \\
\hline \multicolumn{5}{|c|}{ Second Stage Size Reduction } \\
\hline Pulpwood & Hammer mill & $1 / 4$ in. & 5 & 6.5 \\
\hline Wood Residue & Hammer mill & $1 / 4$ in. & 5 & 6.5 \\
\hline
\end{tabular}

\subsubsection{Size Reduction Cost Estimation}

Milling cost estimation is based on vendor-supplied information and equipment performance from typical machine performance and process demonstration unit data and is shown in Table 86.

Table 86. Size reduction cost estimates.

\begin{tabular}{|c|c|c|}
\hline & $\begin{array}{c}2013 \text { SOT } \\
(2011 \$ / \text { dry T) }\end{array}$ & $\begin{array}{c}2017 \text { Target } \\
\text { (2011 \$/dry T) }\end{array}$ \\
\hline & Total & Total \\
\hline \multicolumn{3}{|l|}{ Pulpwood } \\
\hline Chipper & 6.10 & 6.10 \\
\hline Debark/delimb & 6.10 & 6.10 \\
\hline Hammer Mill & 17.09 & 13.97 \\
\hline Total & 29.29 & 26.17 \\
\hline \multicolumn{3}{|l|}{ Wood Residue } \\
\hline Grinder & 5.39 & 5.39 \\
\hline $\begin{array}{l}\text { Trommel } \\
\text { Screen }\end{array}$ & 3.32 & 3.32 \\
\hline Hammer Mill & 17.09 & 13.97 \\
\hline Total & 25.08 & 22.70 \\
\hline
\end{tabular}




\subsubsection{Drying and Densification}

Conversion of lignocellulosic biomass to hydrocarbon fuels: Lignocellulosic Biomass to High Octane Gasoline via Indirect Gasification and Methanol Intermediate use the same technology as fast pyrolysis case described in section 9. Therefore, state of technology and design basis for high moisture technology are the same as fast pyrolysis case described in section 9

A comparison of pellet properties and energy balances for conventional and high-moisture pelletization processes is given in Table 87. The table shows 2017 Design Case targets to achieve a 40 to $50 \%$ reduction in the total pelletization and drying energy.

Table 87. Drying and densification design basis.

\begin{tabular}{|c|c|c|}
\hline & $2013 \mathrm{SOT}$ & 2017 Target \\
\hline Infeed Moisture & $40 \%$ & $30 \%$ \\
\hline Dryer Moisture Reduction & $28 \%$ & $11 \%$ \\
\hline $\begin{array}{l}\text { Densification Moisture } \\
\text { Reduction }\end{array}$ & $3 \%$ & $10 \%$ \\
\hline Final Pellet Moisture & $9 \%$ & $9 \%$ \\
\hline Densification Energy & $75 \mathrm{kWhr} /$ dry T & $50 \mathrm{kWhr} /$ dry $\mathrm{T}$ \\
\hline Drying Energy & $350 \mathrm{kWhr} /$ ton & $100 \mathrm{kWhr} / \mathrm{ton}$ \\
\hline \multicolumn{3}{|l|}{ Pellet Properties } \\
\hline Unit Density & $65 \mathrm{lb} / \mathrm{ft}^{3}$ & $70 \mathrm{lb} / \mathrm{ft}^{3}$ \\
\hline Bulk Density & $35 \mathrm{lb} / \mathrm{ft}^{3}$ & $40 \mathrm{lb} / \mathrm{ft}^{3}$ \\
\hline Durability & Greater than $97.5 \%$ & Greater than $97.5 \%$ \\
\hline
\end{tabular}

The high-moisture densification design basis assumptions are as follows:

- Our preliminary studies indicated that it is possible to produce high-quality pellets woody material; however, for our 2017 Design Case, we are assuming that the process works for other woody and herbaceous feedstocks to produce durable, high-density pellets.

- Technical and cost targets are estimated with the assumption that a grain dryer will be used to dry high-moisture pellets.

- Drying of pellets using energy-efficient driers like grain and belt driers is more economical compared to conventional rotary driers.

- Slow drying at low temperatures of less than $60^{\circ} \mathrm{C}$ can result in more uniform moisture distribution in pellets. 


\subsubsection{Cost Estimation for High-Moisture Densification}

The cost of densification was estimated using vendor-supplied information and the capacity and energy assumptions shown in Table 88.

Table 88. Drying and densification cost estimates

\begin{tabular}{|ccc|} 
& $\begin{array}{c}2013 \text { SOT } \\
(2011 \text { \$/dry T) }\end{array}$ & $\begin{array}{c}2017 \text { Target }(2011 \\
\text { \$/dry T) }\end{array}$ \\
\hline Drying & Total & Total \\
\hline Densification & 17.20 & 5.60 \\
\hline Totals & 7.70 & 4.40 \\
\hline
\end{tabular}

\subsubsection{Formulation/Blending}

To meet feedstock specifications required for various conversion pathways, formulation of specific mixtures of feedstocks will likely be required. Examples include mixing high and lowcost feedstocks to meet cost targets, mixing high and low-ash feedstocks to meet an ash target, mixing of high and low-carbohydrate feedstocks to meet a yield target, and mixing easily and poorly reactive feedstocks to meet a convertibility target. An example of blending to meet an ash and moisture specification is shown in Table 89.

Table 89. Feedstock formulation/blending of ash and moisture contents*.

\begin{tabular}{lccccc|}
$\begin{array}{l}\text { Content Delivered to } \\
\text { Biorefinery Infeed }\end{array}$ & Pulpwood(45\%) & $\begin{array}{l}\text { Wood } \\
\text { Residues(32\%) }\end{array}$ & $\begin{array}{l}\text { Switchgrass } \\
(3 \%)\end{array}$ & $\begin{array}{l}\text { C\&D } \\
\text { waste(20\%) }\end{array}$ & Final Blend \\
\hline $\begin{array}{l}\text { Ash content (wt. \%) } \\
\begin{array}{l}\text { Moisture content (\%, wet } \\
\text { basis) }\end{array}\end{array}$ & 0.5 & 1.0 & 4.0 & 1.0 & $<\mathbf{1 \%}$ \\
HHV (lb/BTU) & 8824 & 9 & 9 & 9 & $\mathbf{9}$ \\
\hline LHV (lb/BTU) & 7255 & 7616 & 6155 & 7255 & $\mathbf{7 3 3 7}$ \\
\hline
\end{tabular}

*Pulpwood, wood resides, and switchgrass composition data were obtained from the INL Biomass Library ${ }^{75}$.

Assumptions for the formulation design basis are as follows:

- Blended feedstocks will be selected and developed to achieve conversion yield specifications. It is currently unknown how blended feedstocks will perform in the conversion pathways. The simplest assumption is the blended feedstocks would be the sum of performances of each individual component. There are on-going trials to test various blended feedstocks and to compare the conversion efficiencies against a single feedstocks.

- Individual feedstocks will be pelleted at depots for shipment to biorefineries. At the biorefinery, these pelleted feedstocks will be unloaded and conveyed into individual bunkers for storage. Pellets of the different blendstocks will be metered out into the bunkers in the 
ratios required of the blends, crushed (using a pellet crusher), and mixed prior to insertion for the conversion process.

- Material will be metered from individual bunkers onto a conveyer and then thoroughly homogenized through this process with no segregation. Mixing of solids occurs in many industries and is often problematic when solids of varying density, shape, and size are blended. This often leads to segregation, either during the mixing or while being transported to its destination. Mixing of solids is considered a trial-and-error process due to these issues.

- The expected unit operations for formulation are shown in Table 90.

Table 90. Feedstock formulation design basis

\begin{tabular}{|lcc|}
\hline 2013 SOT $(2011$ \$/dry T) & Operating Parameters \\
\hline $\begin{array}{l}\text { Pellet Pulverizer } \\
\text { Bulk Storage with }\end{array}$ & 100 ton/hour & Horsepower \\
\hline $\begin{array}{l}\text { Hopper } \\
\text { Conveying System }\end{array}$ & 30 ton/hour & $200 \mathrm{HP}$ \\
\hline
\end{tabular}

Research is currently ongoing at INL to examine the compatibility of various feedstocks blends, with an initial focus on the blends reactivity versus the individual feedstocks. Blends will be developed for several regions of the United States using the least-cost formulation model as a starting point and will incorporate feedstocks with varying levels of reactivity (e.g., herbaceous, woody, and MSW). Reactivity for the fermentation pathway will be investigated first, with expansion into the other DOE conversion pathways in later fiscal years including bio-oil conversion via fast pyrolysis.

While the costs for preprocessing of feedstocks (e.g., grinding, chemical preconversion, pelleting, and drying) are addressed in other parts of the 2017 Design Case, formulation itself will require a different set of preprocessing options in order to match up with the bio-oil conversion pathway. These processes include bulk storage, conveying systems and a pellet pulverizer to insure that the appropriate recipe of material enters the throat of the conversion reactor in the appropriate blends and sizing requirements.

\subsubsection{Cost Estimation for Formulation.}

Cost estimation for formulation was estimated based on existing technology, vendor-supplied information, and equipment performance (Table 91). These costs are cursory and require more extensive research, especially in their specific application to the bioenergy industry. 
Table 91. Formulation cost estimation

\begin{tabular}{|lc|}
\hline & 2017 Target $(2011$ \$ $/$ dry T) \\
\hline Petal \\
\hline Bulk storage with hopper & 1.10 \\
\hline Conveying system & 0.20 \\
\hline Totals & 0.60 \\
\hline
\end{tabular}

\subsubsection{Transportation and Handling Design Basis}

The 2017 Design Case includes formulation and densification meeting feedstock specifications and costs targets. Both processes of formulation and densification will improve feedstock handling operations through active controls. Given formulation and the specific quantities of individual feedstocks required, the average transportation distance will change based on feedstock type. In the 2017 Design Case, pulpwood will be trucked from a local draw radius of 50 miles while switchgrass will be trucked fewer than 15 miles. Error! Reference source not found. in Appendix A shows sufficient C\&D waste resources in the selected counties in western South Carolina therefore C\&D will be transported by truck from transfer stations after processing. This is not a new concept, transfer stations are already used for sorting and transporting valuable material such as cardboard and scrap metal in densified forms (e.g. baled cardboard, crushed and baled scrap metal). Switchgrass will be loaded and unloaded at each location using a loader (telehandler) capable of moving $12 \mathrm{lb} / \mathrm{ft}^{3}$ bales at $20 \%$ respective moisture content. A 53ft. trailer and 800,000-GVW limits were assumed in all trucking operations. Transportation for switchgrass will occur from a field side stack to a densification facility completely separate from the conversion facility, but within a minimal conveyor distance (typically $<50$ miles). C\&D waste transportation will occur from the waste transfer station as pellets to the preprocessing facility for storage and transfer to the biorefinery. Further transportation and handling assumptions are given as follows:

- At 20\% moisture, transportation continues to be volume limited at densities of $12 \mathrm{lb} / \mathrm{ft}^{3}$.

- At 30\% moisture, transportation of chips continues to be volume limited.

- At 9\% moisture, transportation of pellets is weight limited at $40 \mathrm{lb} / \mathrm{ft}^{3}$.

- There will be insignificant material losses throughout transportation and handling.

- Densification will increase material uniformity and flowability

\subsubsection{Cost Estimation for Transportation and Handling}

The cost estimation for transportation and handling was based on vendor-supplied information and equipment performance from typical machines (Table 92). Rail transportation costs were based on work from Searcy using a jumbo hopper car ${ }^{54}$ adjusted for U.S. conditions. 
Table 92. Transportation cost estimates

\begin{tabular}{|c|c|c|}
\hline & $\begin{array}{c}2013 \text { SOT } \\
(2011 \$ / \text { dry T) }\end{array}$ & $\begin{array}{c}2017 \text { Target (2011 } \\
\text { \$/dry T) }\end{array}$ \\
\hline & Total & Total \\
\hline Truck & $14.84^{*}$ & $7.52 * *$ \\
\hline \multicolumn{3}{|c|}{$\begin{array}{l}\text { *Individual feedstock therefore one transportation pathw } \\
* * \text { Multiple feedstocks therefore multiple transportation } \\
\text { pathways and draw radiuses. }\end{array}$} \\
\hline
\end{tabular}

\subsection{Life Cycle Analysis}

This section uses a life-cycle assessment (LCA) to evaluate the energy input and GHG emissions for conversion of Lignocellulosic Biomass to High Octane Gasoline via Indirect Gasification and Methanol Intermediate Pathway. LCA considered the unit processes involved in the biomass logistics. Table 93 shows the energy consumption and Table 94 shows GHG contribution for Lignocellulosic Biomass to High Octane Gasoline via Indirect Gasification and Methanol Intermediate conversion supply chain design.

Table 93. Energy Consumption for Thermochemical conversion supply chain design.

\begin{tabular}{|c|c|c|c|c|c|}
\hline Process Element & Pulpwood & $\begin{array}{l}\text { Wood } \\
\text { Residues }\end{array}$ & Switchgrass & $\begin{array}{l}\text { Construction and } \\
\text { Demolition Waste } \\
\text { (C\&D) }\end{array}$ & Blend \\
\hline Formulation Contribution & $45 \%$ & $32 \%$ & $3 \%$ & $20 \%$ & - \\
\hline $\begin{array}{l}\text { Harvest and collection } \\
(\mathrm{MBTU} / \mathrm{dry} \mathrm{T})\end{array}$ & 182.78 & - & 122.85 & - & 85.94 \\
\hline $\begin{array}{l}\text { Landing Preprocessing/ } \\
\text { Sorting (MBTU/dry T) }\end{array}$ & 231.52 & 110.25 & - & 410.25 & 221.51 \\
\hline $\begin{array}{l}\text { Transportation (MBTU/dry } \\
\text { T) }\end{array}$ & 136.87 & 101.38 & 99.35 & 273.73 & 151.76 \\
\hline Preprocessing (MBTU/dry T) & 408.01 & 408.01 & 285.83 & 408.01 & 404.34 \\
\hline Storage (MBTU/dry T) & 8.46 & 8.46 & 21.83 & 8.46 & 8.86 \\
\hline Handling (MBTU/dry T) & 42.69 & 42.69 & 41.90 & 42.69 & 42.67 \\
\hline $\begin{array}{l}\text { Total Energy Consumption } \\
\text { (MBTU/dry T) }\end{array}$ & 1001.33 & 670.79 & 571.76 & 1143.14 & 915.08 \\
\hline
\end{tabular}


Table 94. GHG contribution for thermochemical conversion supply chain design.

\begin{tabular}{|lc|} 
Process Element & Blend \\
\hline Formulation Contribution & - \\
\hline Harvest and collection GHGs $\left(\mathrm{Kg} \mathrm{CO}_{2} \mathrm{e} /\right.$ dry T) & 8.65 \\
\hline Landing Preprocessing GHGs $\left(\mathrm{Kg} \mathrm{CO}_{2} \mathrm{e} /\right.$ dry T) & 18.95 \\
\hline Transportation GHGs $\left(\mathrm{Kg} \mathrm{CO}_{2} \mathrm{e} /\right.$ dry T) & 8.62 \\
\hline Preprocessing GHGs $\left(\mathrm{Kg} \mathrm{CO}_{2} \mathrm{e} /\right.$ dry T) & 34.60 \\
\hline Storage GHGs $\left(\mathrm{Kg} \mathrm{CO}_{2} \mathrm{e} /\right.$ dry $\left.\mathrm{T}\right)$ & 0.89 \\
Handling GHGs $\left(\mathrm{Kg} \mathrm{CO}_{2} \mathrm{e} /\right.$ dry T) & 4.2 \\
Total GHGs $\left(\mathrm{Kg} \mathrm{CO}_{2} \mathrm{e} /\right.$ dry T) & 75.91
\end{tabular}




\section{References}

1. Lanholtz, M., In Interview, Idaho National Labratory, I., Ed. Oak Ridge National Laboratory (ORNL), 2013.

2. Hess, J. R.; Kenney, K.; Ovard, L.; Searcy, E.; Wright, C. Uniform-Format Solid Feedstock Supply System: A Commodity-Scale Design to Produce an Infrastructure-Compatible Bulk Solid from Lignocellulosic Biomass; Idaho National Laboratory: 2009.

3. Searcy, E.; Hess, J. R. Uniform-Format Feedstock Supply System Design for Lignocellulosic Biomass: A Commodity-Scale Design to Produce an Infrastructure-Compatible Biocrude from Lignocellulosic Biomass; INL/EXT-09-17527; 2009.

4. Argo, A. M.; Tan, E. C. D.; Inman, D.; Langholtz, M. H.; Eaton, L. M.; Jacobson, J. J.; Wright, C. T.; Muth, D. J.; Wu, M. M.; Chiu, Y. W.; Graham, R. L., Investigation of biochemical biorefinery sizing and environmental sustainability impacts for conventional bale system and advanced uniform biomass logistics designs. Biofuels Bioproducts \& Biorefining-Biofpr 2013, 7 (3), 282-302.

5. DOE, U. S.; Perlack, R. D.; Stokes, B. J.; U.S. Billion-Ton Update: Biomass Supply for a Bioenergy and Bioproducts Industry; ORNL/TM-2011/224; 2011.

6. Jones, S.; Meyer, P.; Snowden-Swan, L.; Padmaperuma, A.; Tan, E.; Dutta, A.; Jacobson, J.; Cafferty, K. Process design and economics for the conversion of lignocellulosic biomass to hydrocarbon fuels fast pyrolysis and hydrotreating bio-oil pathway; PNNL-23053; Pacific Northwest National Laboratory (PNNL): 2013.

7. Carpenter, D.; Westover, T. L.; Czernik, S.; Jablonski, W., Biomass feedstocks for renewable fuel production: a review of the impacts of feedstock and pretreatment on the yield and product distribution of fast pyrolysis bio-oils and vapors. Green Chemistry 2014, 16 (2), 384-406.

8. Kenney, K. L.; Smith, W. A.; Gresham, G. L.; Westover, T. L., Understanding biomass feedstock variability; special focus issue. Advanced Feedstocks for Advanced Biofuels 2012, 4 (1), 111-127.

9. Phillips, S. D.; Aden, A.; Jechura, J.; Dayton, D.; Eggeman, T. Thermochemical ethanol via indirect gasification and mixed alcohol synthesis of lignocellulosic biomass; 2007.

10. Raveendran, K.; Ganesh, A.; Khilar, K. C., Influence of mineral matter on biomass pyrolysis characteristics. Fuel 1995, 74 (12), 1812-1822.

11. Drennen, C., Interview. Idaho National Lab, I., Ed. 2013.

12. Searcy, E. M.; Blackwelder, D. B.; Delwiche, M. E.; Ray, A. E.; Kenney, K. L. Validate the cost of feedstock at \$61.57/dry US ton for the production of ethanol via thermochemical conversion; INL/LTD11-24278; Idaho National Laboratory (INL), 2011.

13. Rothbard, M. N., Grain grades and standards - historical issue shaping the future - Hill, L.D. Journal of Economic History 1991, 51 (2), 513-514.

14. Boavida, D.; P. Abelha; I. Gulyurtlu; Valentim, B.; Sousa, M. J. L. D., A study on coal blending for reducing NOx and N2O levels during fluidized bed combustion. Clean Air 2004, 5, 175-191.

15. Jhih-Shyang, S.; Frey, H. C., Coal blending optimization under uncertainty. European Journal of Operational Research 1995, 83 (3), 452-65.

16. Reddy, D. V.; Krishna, N., Precision animal nutrition: A tool for economic and eco-friendly animal production in ruminants. Livestock Research for Rural Development 2009, 21 (3).

17. Sami, M.; Annamalai, K.; Wooldridge, M., Co-firing of coal and biomass fuel blends. Progress in Energy and Combustion Science 2001, 27 (2), 171-214.

18. Walker, J. C. F., Primary wood processing: principles and practice 2nd Edition. Springer: 2006.

19. Phanphanich, M.; Mani, S., Drying characteristics of pine forest residues. Bioresources 2010, 5 (1), 108-120.

20. Hess, J. R.; Foust, T. D.; Wright, L. L.; Sokhansanj, S.; Cushman, J. H.; Easterly, J. L.; Erbach, D. C.; Hettenhaus, J. R.; Hoskinson, R. L.; Sheehan, J. J.; Tagore, S.; Thompson, D. N.; Turhollow, A. Roadmap for agriculture biomass feedstock supply in the United States; U.S. Department of Energy, Energy Efficiency and Renewable Energy: 2003. 
21. Templeton, D.; Sluiter, A.; Hayward, T.; Hames, B.; Thomas, S., Assessing corn stover composition and sources of variability via NIRS. Cellulose 2009, 16 (4), 621-639.

22. Shinners, K. J.; Boettcher, G. C.; Muck, R. E.; Weimer, P. J.; Casler, M. D., Harvest and storage of two perennial grasses as biomass feedstocks. Transactions of the ASABE 2010, 53 (2), 359-370.

23. Turn, S. Q.; Kinoshita, C. M.; Ishimura, D. M., Removal of inorganic constituents of biomass feedstocks by mechanical dewatering and leaching. Biomass Bioenerg. 1997, 12 (4), 241-252.

24. Karlen, D. L.; Muth, D. J., Landscape management for sustainable supplies of bioenergy feedstock and enhanced soil quality. Agrociencia Uruguay, Special Issue 2012.

25. Adler, P. R.; Sanderson, M. A.; Boateng, A. A.; Weimer, P. I.; Jung, H. J. G., Biomass yield and biofuel quality of switchgrass harvested in fall or spring. Agronomy Journal 2006, 98 (6), 1518-1525.

26. Hoskinson, R. L.; Karlen, D. L.; Birrell, S. J.; Radtke, C. W.; Wilhelm, W. W., Engineering, nutrient removal, and feedstock conversion evaluations of four corn stover harvest scenarios. ScienceDirect 2007, (31), 126-136.

27. Wilhelm, W. W.; Johnson, J. M. F.; Lightle, D. T.; Karlen, D. L.; Novak, J. M.; Barbour, N. W.; Laird, D. A.; Baker, J.; Ochsner, T. E.; Halvorson, A. D.; Archer, D. W.; Arriaga, F., Vertical distribution of corn stover dry mass grown at several US locations. BioEnergy Research 2011, 4 (1), 11-21.

28. Hess, J. R.; Wright, C. T.; Kenney, K. L., Cellulosic biomass feedstocks and logistics for ethanol production. Biofuels Bioproducts \& Biorefining-Biofpr 2007, I (3), 181-190.

29. NREL Process Design and Economics for Biochemical Conversion of Lignocellulosic Biomass to Ethanol; NREL/TP-5100-47764; September, 2011.

30. Roser, D.; Mola-Yudego, B.; Sikanen, L.; Prinz, R.; Gritten, D.; Emer, B.; Vaatainen, K.; Erkkila, A., Natural drying treatments during seasonal storage of wood for bioenergy in different European locations.

Biomass Bioenerg. 2011, 35 (10), 4238-4247.

31. Kim, D.-W.; Murphy, G., Forecasting air-drying rates of small douglas-fir and hybrid poplar stacks in oregon, USA. International Journal of Forest Engineering 2013, 24 (2), 137-147.

32. He, X.; Lau, A. K.; Sokhansanj, S.; Lim, C. J.; Bi, X. T.; Melin, S., Dry matter losses in combination with gaseous emissions during the storage of forest residues. Fuel 2012, 95 (1), 662-664.

33. Jirjis, R., Effects of particle size and pile height on storage and fuel quality of comminuted Salix viminalis. Biomass Bioenerg. 2005, 28 (2), 193-201.

34. Watson, W. F.; Twaddle, A.; Hudson, J. B., Review of chain flail delimbing-debarking. International Journal of Forest Engineering 1993, 4 (2), 37-51.

35. Jirjis, R., Storage and drying of wood fuel. Biomass Bioenerg. 1995, 9 (1-5), 181-190.

36. Ferrero, F.; Malow, M.; Noll, M., Temperature and gas evolution during large scale outside storage of wood chips. European Journal of Wood and Wood Products 2011, 69 (4), 587-595.

37. Noll, M.; Jirjis, R., Microbial communities in large-scale wood piles and their effects on wood quality and the environment. Applied Microbiology and Biotechnology 2012, 95 (3), 551-563.

38. Searcy, E. M.; Blackwelder, D. B.; Delwiche, M. E.; Ray, A. E.; Kenney, K. L., Impact of Screening on Behavior during Storage and Cost of Ground Small-Diameter Pine Trees: A Case Study. Forest Products Journal 2011, 61 (7), 570-578.

39. Fuller, W. S., Chip pile storage - a review of practices to avoid deterioration and economic - losses.

Tappi Journal 1985, $68(8), 48-52$.

40. Schutte-Buffalo, H. M. http://www.hammermills.com/size-reduction-product-categories-schuttebuffalo-hammermill.

41. Yancey, N. A.; Tumuluru, J. S.; Wright, C. T., Drying, Grinding and Pelletization Studies on Raw and Formulated Biomass Feedstock's for Bioenergy Applications. Journal of Biobased Materials and Bioenergy 2013, 7 (5), 549-558.

42. Tumuluru, J. S.; Sokhansanj, S.; Hess, J. R.; Wright, C. T.; Boardman, R. D., A review on biomass torrefaction process and product properties for energy applications. Industrial Biotechnology 2011, 7 (5), 384-401.

43. DOE, U. S. Bioenergy Technologies Office Peer Review 2013. 
44. Davis, D.; Tao, L.; Tan, E.; Biddy, M.; Beckham, G.; Scarlata, C.; Jacobson, J.; Cafferty, K.; Ross, J.; Lukas, J.; Knorr, D.; Schoen, P. Process design and economics for the conversion of lignocellulosic biomass to hydrocarbons: dilute-acid and enzymatic deconstruction of biomass to sugars and biological conversion of sugars to hydrocarbons; Natioanl Renewable Energy Laboratory: Golden, CO, 2013. 45. CENNATEK Feasibility of improving biomass combustion through extraction of nutrients; $2011 ; \mathrm{p}$ 107.

46. Humbird, D.; Davis, R.; Tao, L.; Kinchin, C.; Hsu, D.; Aden, A.; Schoen, P.; Lukas, J.; Olthof, B.; Worley, M.; Sexton, D.; Dudgeon, D. Process design and economics for biochemical conversion of lignocellulosic biomass to ethanol; 2011.

47. Langholtz, M.; Graham, R.; Eaton, L.; Perlack, R.; Hellwinkel, C.; Ugarte, D. G. D. 1. T., Price projections of feedstocks for biofuels and biopower in the U.S. Energy Policy 2012, 41, 484-493.

48. Bonner, I. J.; Kenney, K. L., Moisture sorption characteristics and modeling of energy sorghum (Sorghum bicolor (L.) Moench). Journal of Stored Products Research 2013, 52, 128-136.

49. Iowa-State-University, Agriculture and Natural Resources. 2013.

50. Yu, M.; Zeng, G.; Chen, Y.; Yu, H.; Huang, D.; Tang, L., Influence of Phanerochaete chrysosporium on microbial communities and lignocellulose degradation during solid-state fermentation of rice straw.

Process Biochemistry 2009, 44 (1), 17-22.

51. Selig, M.; Weiss, N.; Ji, Y. Enzymatic saccharification of lignocellulosic biomass: LAP NREL/TP510-42629 2008.

52. Dowe, N.; McMillan, J. SSF experimental protocols — lignocellulosic biomass hydrolysis and fermentation; NREL/TP-510-42630; 2001.

53. Shi, J.; Thompson, V.; Yancey, N.; Stavila, V.; Simmons, B. A.; Singh, S., Impact of mixed feedstocks and feedstock densification on ionic liquid pretreatment efficiency. Biofuels 2013, 4 (1), 63 72.

54. Searcy, E.; Flynn, P.; Ghafoori, E.; Kumar, A., The relative cost of biomass energy transport. Applied Biochemistry and Biotechnology 2007, 137, 639-652.

55. PNNL Process design and economics for the conversion of lignocellulosic biomass to hydrocarbon fuels; November 2013.

56. Service, U. S. F. Forest Resources of United States, 2012. http://www.fia.fs.fed.us/programfeatures/rpa/docs/2012\%20RPA_\%20Review\%20Draft\%20Resource\%20Tables\%2002-18-2014.pdf. 57. Idaho National Lab, I. Feedstock Supply System Design and Economics for Conversion of Lignocellulosic Biomass to Hydrocarbon Fuels - Conversion Pathway: Biological Conversion of Sugars to Hydrocarbons: The 2017 Design Case; Idaho Falls, Idaho USA: Idaho National Laboratory, 2013. 58. Taylor, S.; Bob, R.; Corley, F.; Gallagher, T.; Fasina, O.; McDonald, T.; Smidt, M., High tonnage forest biomass from southern pine. Auburn University: 2012.

59. Werther, J.; Saenger, M.; Hartge, E. U.; Ogada, T.; Siagi, Z., Combustion of agricultural residues. Progress in Energy and Combustion Science 2000, 26 (1), 1-27.

60. Lindstroem, E.; Oehman, M.; Backman, R.; Bostroem, D., Influence of sand contamination on slag formation during combustion of wood derived fuels. Energy \& Fuels 2008, 22 (4), 2216-2220. 61. Ahmad, M.; Roberts, J.; Hardiman, E.; Singh, R.; Eltis, L.; Bugg, T., Identification of DypB rhodococcus jostii RHA1 as a lignin perozidase. Biochemistry 2011, 50, 5096-5107.

62. Das, K. C.; Singh, K.; Bibens, B.; Hilten, R.; Baker, S. A.; Greene, W. D.; Peterson, J. D., Pyrolysis characteristics of forest residues obtained from different harvesting methods. Applied Engineering in Agriculture 2011, 27 (1), 107-113.

63. Bartels, D.; Sunkar, R., Drought and salt tolerance in plants. Critical Reviews in Plant Sciences 2005, 24 (1), 23-58.

64. Cutshall, J.; Greene, D.; Baker, S.; Mitchell, D., Transpirational drying effects on energy and ash content from whole-tree chipping operations in a southern pine plantation. 34th Council on Forest Engineering 2011. 
65. Dukes, C. C.; Baker, S. A.; Greene, W. D., In-wood grinding and screening of forest residues for biomass feedstock applications. Biomass Bioenerg. 2013, 54, 18-26.

66. Baker, S. A.; Westbrook, M. D., Jr.; Greene, W. D., Evaluation of integrated harvesting systems in pine stands of the southern United States. Biomass Bioenerg. 2010, 34 (5), 720-727.

67. Smidt, M.; Tufts, R.; Gallagher, T., Logging efficiency and cost. In ANR-1347 - Alabama

Cooperative Extension System, 2009.

68. Hiesl, P.; Benjamin, J. G., Applicability of international harvesting equipment productivity studies in maine, USA: a literature review. Forests 2013, 4 (4), 898-921.

69. Jones, P. D.; Grado, S. C.; Demarais, S., Financial analysis of intensive pine plantation establishment. Journal of Forest Economics 2010, 16 (2), 101-112.

70. Cunningham, K.; Barry, J.; Walkingstick, T. Managing loblolly pine stands... a to z; FSA5023; University of Arkansas Division of Agriculture, Director, Cooperative Extension Service, University of Agriculture, Cooperative Extension Service

Arkansas, December 2013, 2013.

71. McLaughlin, S. B.; Kszos, L. A., Development of switchgrass (Panicum virgatum) as a bioenergy feedstock in the United States. Biomass Bioenerg. 2005, 28 (6), 515-535.

72. Smith, W. A.; Bonner, I. J.; Kenney, K. L.; Wendt, L. M., Practical considerations of moisture in baled biomass feedstocks. Biofuels 2013, 4 (1), 95-110.

73. Cundiff, J. S.; Marsh, L. S., Harvest and storage costs for bales of switchgrass in the southeastern United States. Bioresource Technology 1996, 56 (1), 95-101.

74. Darr, M. J.; Shah, A., Biomass storage: and update on industrial solutions for baled biomass feedstocks. Biofuels 2012, 3 (3), 321-332.

75. Idaho National Laboratory, I. Biomass Analytical Library.

https://inlportal.inl.gov/portal/server.pt?open=514\&objID=1350\&mode=2.

76. News, W. R. July 20, 2012.

77. Valkenburg, C.; Gerber, M. A.; Walton, C. W.; Jones, S. B.; Thompson, B. L.; Stevens, D. J., Municipal solid waste (MSW) to liquid fuels synthesis. Availability of feedstock and technology, 2008, 1 (PNNL-18144).

78. Shi, J.; Ebrik, M.; Yang, B.; Wyman, C., The potential of cellulosic ethanol production from municipal solid waste: a technical and economic evaluation. University of California Energy Institute: Berkeley, California, 2009.

79. Gustafson, R.; Bura, R.; Cooper, J.; McMahon, R.; Schmitt, E.; Vajzovic, A., Converting Washington lignocellulosic rich urban waste to ethanol. Ecology Publication: Washington State University 2009, 09$07-060$.

80. Yan, S.; Yao, J.; Yao, L.; Zhi, Z.; Chen, X.; Wu, J., Fed batch enzymatic saccharification of food waste improves the sugar concentration in the hydrolysates and eventually the ethanol fermentation by Saccharomyces cerevisiae H058. Brazilian Arch. Biol. Technology 2012, 55 (2), 183-192.

81. Idaho National Lab, I. Unpublished data generated at INL.

82. Cho, D. H.; Shin, S. J.; Bae, Y.; Park, C.; Kim, Y. H., Ethanol production from acid hydrolysates based on the construction and demolition wood waste using Pichia stipitis,. Bioresource Technology 2011, 102, 4439-4443.

83. Gresham, G. L.; Emerson, R.; Hoover, A.; Miller, A.; Kenney, K. L. Evolution and Development of Effective Feedstock Specifications; Milestone Completion Report, \#ID 2.1.1.1.A.ML.3; Idaho National Laboratory, 2013.

84. Jenkins, B. M., Biomass leachate treatment by reverse osmosis. Fuel Processing Technology 2003, 81, 223-246.

85. ASABE ASABE Standards: Agricultural Machinery Management Data. . http://asae.frymulti.com/standards.asp. 


\section{Appendix A \\ Construction \& Demolition Waste and Municipal Solid Waste}

\section{Construction \& Demolition Waste}

Construction and Demolition (C\&D) waste is a potential feedstock for the thermochemical pathways. This stream consists of waste materials generated during construction, renovation, and demolition from both residential and non-residential sources. In a 2009 report (EPA530-R-09002), the Environmental Protection Agency (EPA) estimated that approximately 170 million tons of C\&D waste was generated in 2003 in the United States, going to an EPA-estimated 1,900 C\&D landfills, although more recently many localities are setting recycling targets for C\&D projects (http://www.nyc.gov/html/ddc/downloads/ pdf/waste.pdf). The composition of this waste stream is primarily wood, drywall, metal, plastics, roofing, masonry, glass, cardboard, concrete, and asphalt debris. The relative amounts of these materials vary greatly depending on the relative percentages of new construction versus renovation and demolition, as well as the type and size of structures being built, renovated, or demolished. The only fraction relevant to a biorefinery would be the woody material that consists of both untreated and treated (e.g., painted, stained, or varnished) materials. It is currently unknown whether the treated material would affect downstream processing of these materials in a thermochemical process.

C\&D waste generally is not part of the residential MSW stream and is handled by construction contractors. In some locations, onsite sorting occurs by the contractors and the untreated woody fraction would be readily available. An internet survey of landfills and transfer stations showed that those facilities will only receive untreated woody material and generally compost these materials. These facilities also would be a source for this material. In areas where onsite sorting does not occur, some type of sorting to remove non-woody materials would be required. In the given study area potential C\&D waste availability was determined by the South Carolina Solid Waste Management Annual Report 2012. (Error! Reference source not found.).

Table A-1 Potential C\&D available in select counties in western South Carolina

\begin{tabular}{|lc|}
\hline \multicolumn{1}{|c}{ County } & $\begin{array}{c}\text { Potential C\&D Waste } \\
\text { (tons) }\end{array}$ \\
\hline Aiken & 32553.2 \\
\hline Edegfield & 2406 \\
\hline Greenwood & 3688.4 \\
\hline Kershaw & 13766.8 \\
\hline Laurens & 7504.4 \\
\hline Lexington & 52146.4 \\
\hline Newberry & 3335.6 \\
\hline Richland & 79640.8 \\
\hline Saluda & 150.4 \\
\hline Total & 195192 \\
\hline
\end{tabular}




\section{Municipal Solid Waste}

MSW is attractive as a feedstock because it is available year-round, it already has an established infrastructure for collection and handling, and it has the potential to be low cost. MSW currently is a negative cost feedstock because municipalities paid an average of $\$ 49.27 /$ ton in 2012 , with a range from $\$ 18.43$ in Idaho to $\$ 105.40$ in Massachusetts for landfilling ${ }^{76}$. While it is unlikely that MSW will be available to the biorefinery at negative costs because MSW will require processing to separate out the fractions of interest and will require other types of preprocessing to upgrade the quality, it likely will still be available at lower cost than other herbaceous feedstocks. An average composition of MSW is provided in Table A-2.

Table A- 2 National average municipal solid waste composition.

\begin{tabular}{|lc|}
\hline \multicolumn{1}{c}{ Material } & \% Total MSW \\
\hline Paper and paperboard & $28.5 \%$ \\
\hline Glass & $4.6 \%$ \\
\hline Steel & $6.8 \%$ \\
\hline Aluminum & $1.4 \%$ \\
\hline Other nonferrous metals & $0.8 \%$ \\
\hline Plastics & $12.4 \%$ \\
\hline Rubber and leather & $3.1 \%$ \\
\hline Textiles & $5.3 \%$ \\
\hline Wood & $6.4 \%$ \\
\hline Other materials & $1.9 \%$ \\
\hline Food & $13.9 \%$ \\
\hline Yard trimmings & $13.4 \%$ \\
\hline Misc. inorganic waste & $1.5 \%$ \\
\hline
\end{tabular}

Candidate materials for the biochemical pathway include paper and paperboard, food, and yard waste. Of these, paper and paperboard are likely to have more value when recycled than as a feedstock for fuels; however, there is still a significant fraction of paper and paperboard that is non-recyclable, including coated paper and cardboard, polycoat material, glossy papers such as magazines, food-contaminated papers and cardboards, and any material with binders such as phone books.

Table A-2 shows generation rates for these fractions for 14 different state and/or regions. Of these fractions, food waste has the highest rate of generation and will be available year-round. Non-recyclable paper has the next highest generation rate and also would be available yearround. Yard waste has the lowest rate of generation and may not be available year-round depending on location. 
Table A- 3. Per capita generation rates for various fractions of municipal solid waste and construction and demolition waste (lb/person/day).

\begin{tabular}{lccrr}
\hline \multicolumn{1}{c}{ Location } & Yard waste & Food waste & $\begin{array}{c}\text { Non-recyclable } \\
\text { paper }\end{array}$ & $\begin{array}{c}\text { Untreated wood } \\
\text { C\&D waste }\end{array}$ \\
\hline AZ - Phoenix ${ }^{1}$ & 0.40 & 0.29 & 0.11 & 0.03 \\
CO - Boulder & 0.52 & 0.58 & 0.33 & 0.07 \\
Co. & 0.19 & 0.39 & 0.34 & 0.12 \\
CO - Larimer & & & & 0.10 \\
Co. & 0.27 & 0.50 & 0.44 & 0.38 \\
CT & 0.46 & 0.66 & 0.61 & 0.09 \\
DE & 0.16 & 0.72 & 0.16 & 0.22 \\
HI & 0.18 & 0.53 & 0.40 & 0.15 \\
IA & 0.14 & 0.95 & 0.47 & 0.15 \\
IL & 0.17 & 0.89 & 0.47 & 0.06 \\
MA -eastern & 0.10 & 0.40 & 0.19 & 0.15 \\
MA-central & 0.07 & 0.41 & 0.40 & 0.24 \\
MN & 0.06 & 0.50 & 0.52 & 0.14 \\
PA & 0.17 & 0.54 & 0.22 & 0.61 \\
WA & 0.06 & 0.49 & 0.41 & 0.18 \\
WI & 0.21 & 0.56 & 0.36 & \\
Average & & &
\end{tabular}

Other considerations for these MSW fractions include moisture content, ash content, carbohydrate content, compatibility with other biorefinery operations, and obtaining a clean feed stream of these fractions from mixed MSW (Table A-4). 
Table A- 4. Physical parameters of solid waste

\begin{tabular}{|c|c|c|c|c|c|}
\hline Fraction & Moisture (\%) & $\begin{array}{l}\text { Ash } \\
(\%)\end{array}$ & $\begin{array}{l}\text { Carbohydrate }(\%) \\
\text { (glucan+xylan) }\end{array}$ & Pretreatment Severity & $\begin{array}{l}\text { Sorting } \\
\text { Required? }\end{array}$ \\
\hline Yard waste & $43^{77}$ & $28^{78}$ & 46 & $\begin{array}{c}\text { More severe } \\
\text { pretreatment may be } \\
\text { needed }\end{array}$ & $\begin{array}{l}\text { No, if curbside } \\
\text { recycling is in } \\
\text { place }\end{array}$ \\
\hline Food waste & $37^{77}$ & NA & 64 & No pretreatment needed & Yes \\
\hline $\begin{array}{c}\text { Non-recyclable } \\
\text { paper }\end{array}$ & 5 & $19.5^{79}$ & 56 & $\begin{array}{c}\text { Lower severity } \\
\text { pretreatment needed }\end{array}$ & Yes \\
\hline $\begin{array}{l}\text { Untreated C\&D } \\
\text { wood }\end{array}$ & $13^{77}$ & $6.5^{78}$ & 62 & $\begin{array}{l}\text { Higher severity } \\
\text { pretreatment required }\end{array}$ & $\begin{array}{l}\text { Yes, unless } \\
\text { onsite sorting } \\
\text { occurs }\end{array}$ \\
\hline \multicolumn{6}{|c|}{${ }_{78}^{1}$ Valkenburg et al. $2008{ }^{77}$} \\
\hline \multicolumn{6}{|c|}{$\begin{array}{l}{ }^{3} \text { Gustafson et al. } 2009{ }^{79} \\
{ }^{4} \text { Yan et al. } 2012{ }^{80} \\
{ }^{5} \text { Unpublished data generat }\end{array}$} \\
\hline
\end{tabular}

The non-recyclable paper and untreated C\&D wood are both below the target moisture content and can be readily blended with other herbaceous materials. With a final ash specification of $<1 \%$ for the blended feedstock, only the C\&D waste that has been treated by a wash stage could be used if blended with lower ash materials. It is estimated that the wash stage would reduce ash content down to about $1 \%$ and cost about $\$ 4.15 /$ dry T making its application still cost effective. 


\section{Appendix B \\ Least Cost Formulation}

The biofuel conversion quality in-feed specifications have a large impact on whether or not a particular feedstock is cost effective. Raw biomass standing in the field is aerobically unstable, high in moisture and does not qualify as a feedstock; certain upgrades to the raw biomass are needed to make it compatible with conversion requirements. In addition, raw biomass is highly variable even within a single field.

In response to the variability in biomass quality, a variety of more robust biofuel conversion technologies are being developed, even though it is unlikely a single best conversion technology will be capable of handling all the variability experienced within raw biomass. Other approaches to addressing the variability include blending/formulation, leaching, densification, and other preprocessing options.

By combining analyses using farm gate price assumptions with quality specifications obtained from the Biomass R\&D Library, gains in the projected volumes available at cost and biorefinery specifications are being realized by transitioning to a blended feedstock approach. Feedstock blending allows a conversion facility to collect less of any one feedstock and thus pay a lower average price for each feedstock by moving down the cost vs. supply curve.

In addition, with blended feedstocks biomass quality is a key aspect to consider when analyzing cost and volume availability. Formulating a designed feedstock through blending and other preprocessing logistical methods allows low cost and typically low quality biomass to be blended with biomass of higher cost and typically higher quality to achieve the specifications at the infeed of a conversion facility. The use of low cost biomass allows the supply chain to implement additional preprocessing technologies that actively control feedstock quality, while also bringing more biomass into the system. This analysis and design approach is being called the "least-cost formulation" (LCF) strategy.

The LCF concept is best explained through an example of how it is calculated. This analysis focuses on the baseline scenario located in western Kansas to illustrate the least-cost formulation approach to resource selection for the 2017 Design Case. This approach challenges the singlefeedstock paradigm by allowing multiple available resources to compete based on cost, quantity, and quality considerations. It ultimately is demonstrated that such an approach can contribute significant cost reductions to biomass feedstock supply. Note that it is assumed that a blended feedstock will perform "like" the single feedstock. There are a number of collaboration projects between the INL and NREL and PNNL to test these blended feedstocks and evaluate their performance compared to single feedstocks.

At present most cellulosic biomass feedstock supply systems are designed around a single feedstock, typically corn stover. Figure B1 illustrates the available corn stover and switchgrass at varying farm gate prices for the western Kansas scenario. Note that these supply curves represent the projected cost (i.e., farm gate) and quantity available in 2017 based on data from The Billion Ton Update ${ }^{5}$. In order to account for losses throughout the supply chain, particularly dry matter 
losses in storage, a total of 870,000 dry $\mathrm{T}$. of biomass must be sourced in order to deliver 800,000 dry T. to the biorefinery. According to the farm gate supply curves in Figure B1, sufficient quantities of both corn stover and switchgrass are available in this area at a cost of $\$ 49$ and $\$ 57 /$ dry $\mathrm{T}$ for corn stover and switchgrass, respectively. These farm gate supply curves indicate that even though switchgrass is available in this area, it cannot compete with the lower cost of corn stover.

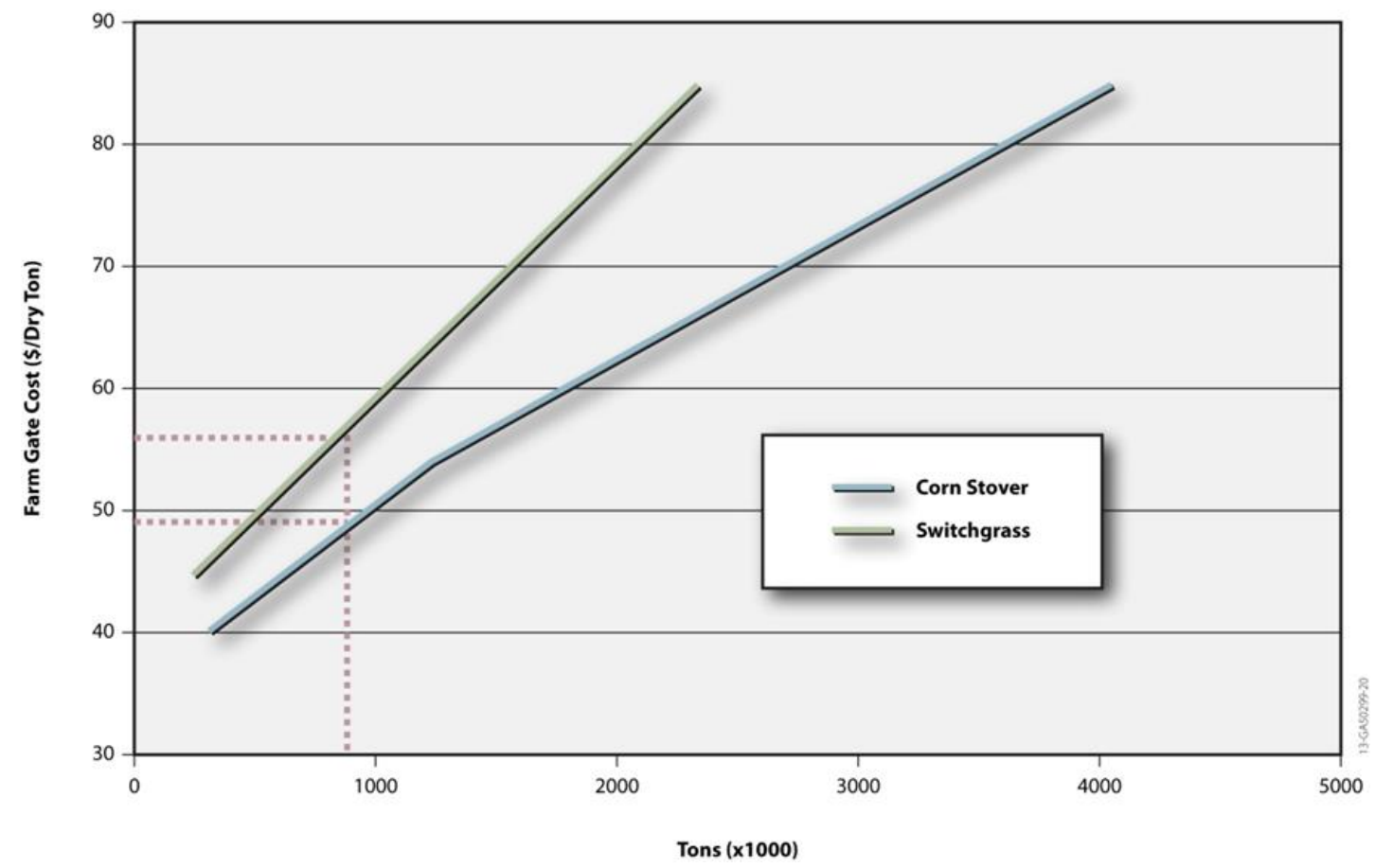

Figure B-1. The farm gate cost curves suggest that corn stover is the preferred feedstock because it is less expensive than switchgrass

However, when we consider the total delivered feedstock costs, which includes farm gate price plus logistics costs plus quality dockage costs then the dynamics of the biomass supply curve begin to change. These additional costs shift the aggregated supply curves to the left; the addition of a dockage cost differs for corn stover and switchgrass because corn stover on average has a higher ash content, so the curves do not shift by the same amount (Figure B2). Logistics costs for switchgrass are lower primarily because of the higher yields, lower moisture, and improved preprocessing characteristics. In addition, the higher moisture and ash content of corn stover results in quality dockage costs (both ash and convertibility) for corn stover, where no dockage is applied to switchgrass. The result is that corn stover costs increase relative to switchgrass (Figure B2). Considering the total delivered feedstock costs, corn stover and switchgrass could each be supplied at the 870,000 ton quantity for about $\$ 84$ and $\$ 85 /$ dry $T$, respectively. This is only a $\$ 1 /$ ton difference compared to the $\$ 8 /$ ton difference when only farm gate price was considered. 


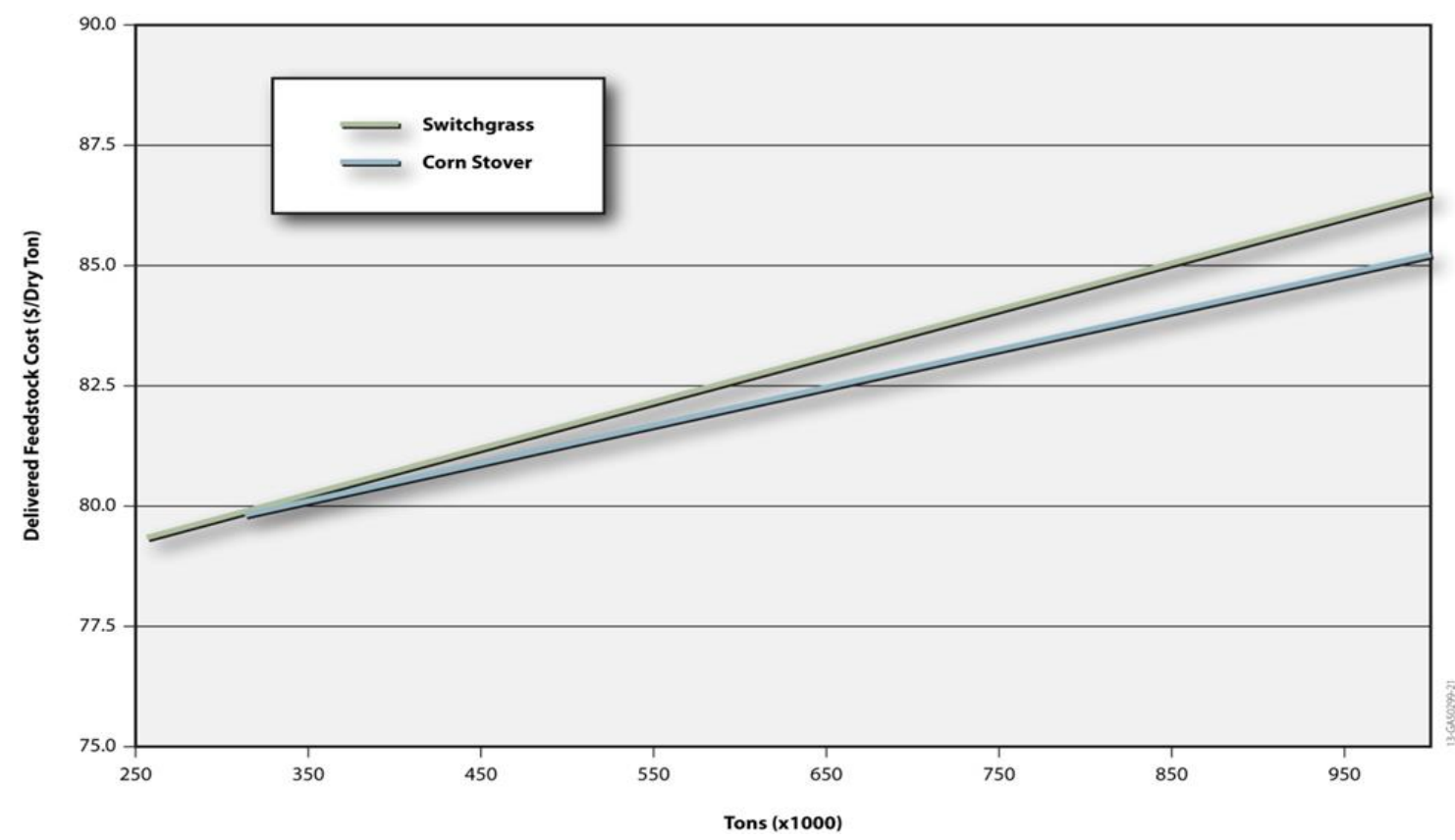

Figure B-2. Accounting for quality (dockage) — shows that about 300,000 tons of switchgrass can be supplied at a lower cost than corn stover

The feedstock supply curves in Figure B2 identify an opportunity for those not wed to a single feedstock. These supply/demand curves indicate that about 300,000 tons of switchgrass can be sourced at a lower cost than corn stover; however, beyond this amount, corn stove once again is more affordable. This gives rise to the least-cost formulation or blended feedstock strategy, which, in this case, replaces higher cost corn stover with lower cost switchgrass. By sourcing 300,000 tons of switchgrass and 550,000 tons of corn stover, a corn stover/switchgrass blend can be supplied and delivered at about $\$ 81 /$ dry T, compared to $\$ 84 /$ dry T for corn stover and $\$ 85 /$ dry T for switchgrass (Figure B3) 


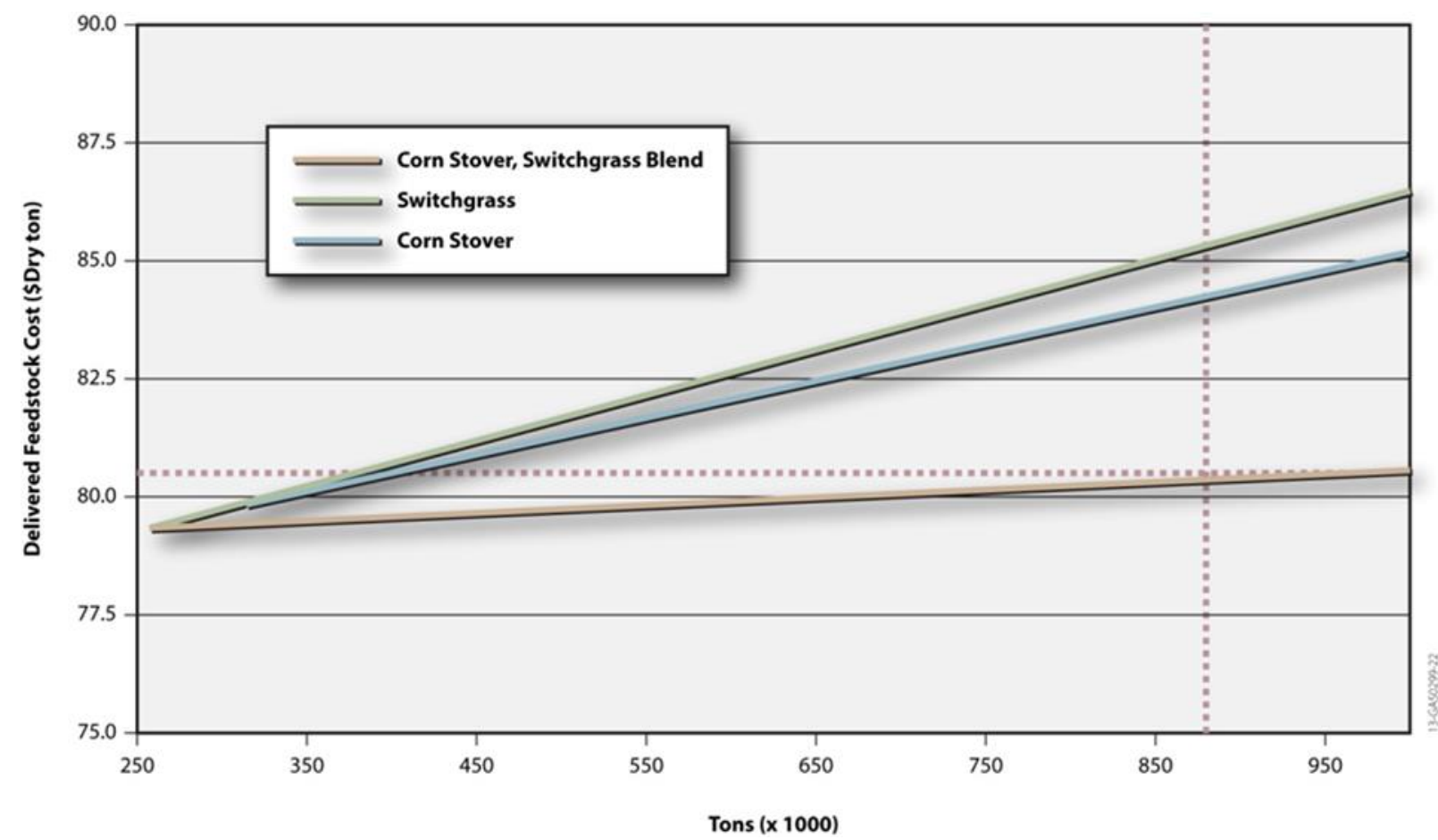

Figure B-3. A corn stover/switchgrass blend that will deliver at about $\$ 81 /$ dry T

The least-cost formulation strategy suggests that further reducing feedstock costs, beyond what can be attained with the corn stover/switchgrass blend requires additional blendstock alternatives that can be accessed at lower costs than either switchgrass or corn stover. It is at this point that we introduce the potential of Municipal Solid Waste (MSW) as a low-cost feedstock alternative. MSW is discussed in detail in

Appendix A, where it is suggested that several MSW fractions are likely available at sufficiently low cost to be attractive blendstocks. Assuming an average access cost of $\$ 18 / \mathrm{dry}^{78}$ and logistics costs of about $\$ 44 /$ ton, MSW can be delivered for about $\$ 62 /$ ton. At this cost, approximately $5 \%(44,000$ dry T) of MSW added to the corn stover/switchgrass blend is sufficient to reduce the delivered feedstock costs an additional $\$ 1$ to achieve the $\$ 80 /$ dry T target (Figure B4). Recognizing that much uncertainty currently exists about the cost, availability, and conversion performance of MSW, a 5\% MSW blend that contributes about $\$ 1 /$ dry T to the $\$ 80 /$ dry T target seems an acceptable level of risk until more research is completed to support higher blend levels 


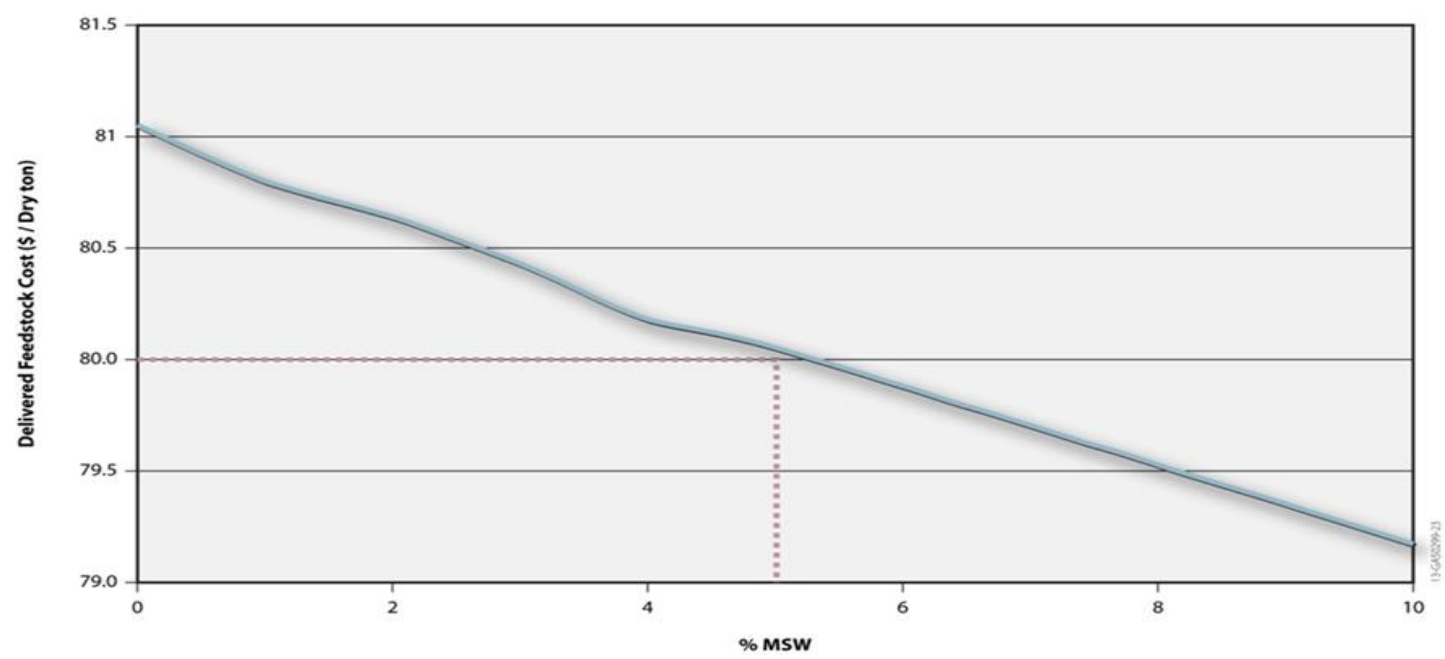

Figure B-4. A minimum of 5\% MSW (at $\$ 1 /$ dry T) is needed to achieve the $\$ 80 /$ dry $\mathrm{T}$ cost target with a corn stover, switchgrass, and municipal solid waste blend

The supply curves in Figure B5 present the options available to a biorefinery in an area where a single, highly abundant, low-cost feedstock is not available. In the 2017 Design Case, the $\$ 80$ feedstock cost target is only achieved by accessing multiple resources, including MSW. The least-cost formulation approach resulted in a feedstock blend consisting of $60 \%(522,000$ dry $\mathrm{T})$ corn stover, 35\% (304,500 dry T) switchgrass, and 5\% (43,500 dry T) sorted MSW.

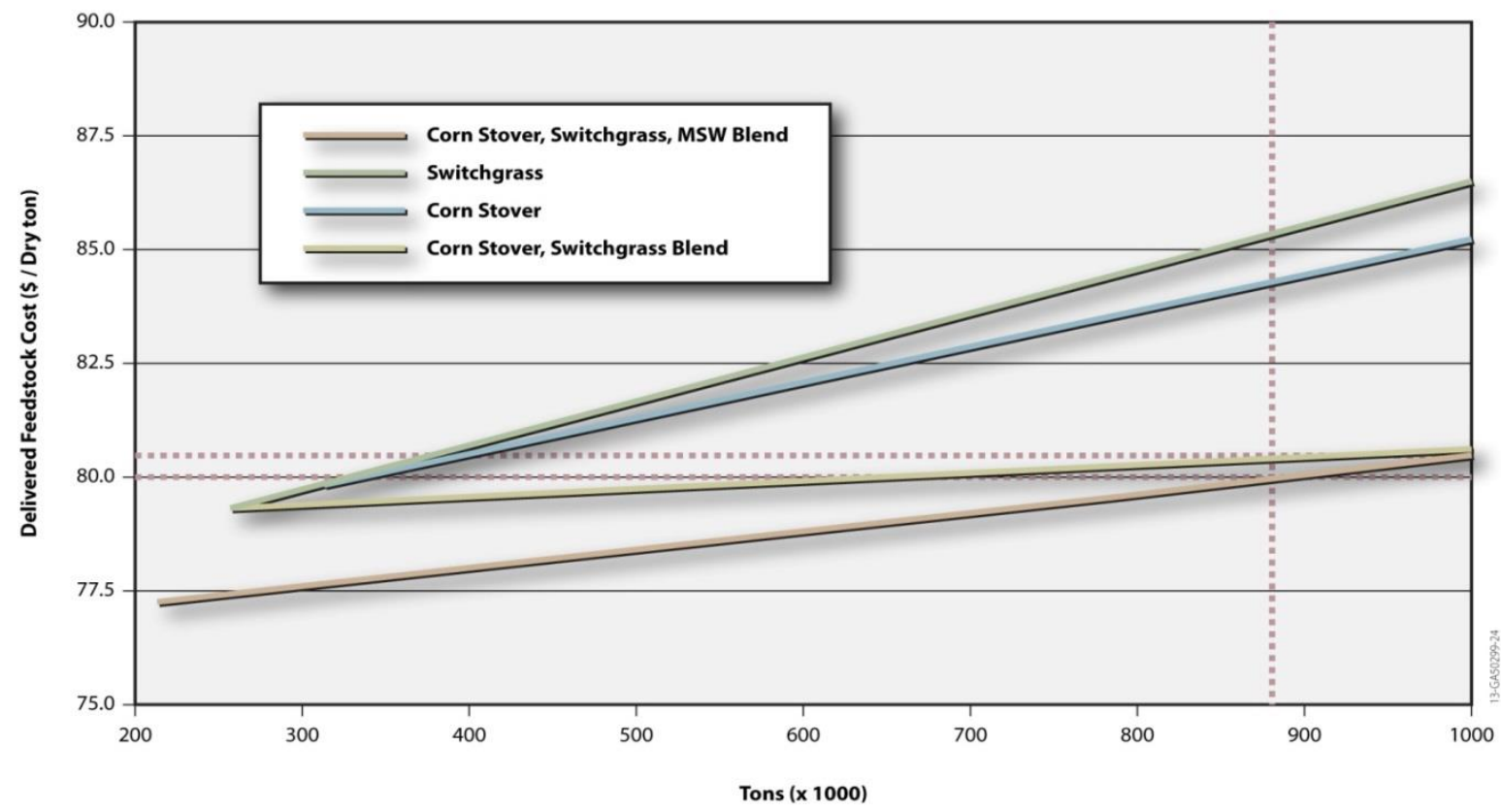

Figure B-5. Comparison of individual and blended feedstock costs. A blend of $60 \%$ corn stover, $35 \%$ switchgrass, and 5\% municipal solid waste is needed to hit the $\$ 80$ feedstock cost target 
The availability of these resources for the western Kansas scenario that was chosen to demonstrate the 2017 Design Case is illustrated in Figure 18. Corn stover and switchgrass are available within a 35-mile supply radius of the biorefinery. The source of MSW generally is tied to human generation; therefore, even 5\% MSW requires a rather sizeable human population. This means that the MSW supply associated with this scenario comes out of the Denver, Colorado metropolitan area. It is not unusual for large metropolitan areas to ship their MSW to distant landfills; therefore, this scenario is likely replicable to many areas around the country. 


\section{Appendix C \\ Off-Specifications Feedstock Dockage Approach}

Ash serves no purpose in a conversion process, and in fact will result in additional costs for disposal, machine wear, and potential negative implications on the process itself. In order to mitigate these costs, they must be subtracted from the purchase payment of biomass that contains unacceptable levels of ash contamination. This milestone report demonstrates the potential impact of off-specification ash content in a traditional, well vetted liquid fuel conversion system.

The analysis below was based on the costs ${ }^{83}$ where feedstock procurement is priced at 58.50

$\$ / D M T$. Disposal of ash was applied at $28.86 \$ /$ dry T of ash. The ethanol conversion yield of 79 $\mathrm{gal} / \mathrm{dry} \mathrm{T}$ was used to estimate non-enzymatic manufacturing costs and capital costs at 23.94 $\$ /$ dry T and $13.83 \$ /$ dry T, respectively. The analysis assumes that ash content is measured at the point of sale between a feedstock supplier and refinery (exchange-point), and payout is based on the delivered material's quality compared to a baseline feedstock containing $5 \%$ ash. It was assumed that production quality and quantity must be maintained, such that the decreased yield resulting from off-specification (high ash) feedstock will require additional biomass to be purchased and processed to match the yield anticipated from baseline feedstocks. Five individually calculated dockages encompass the reduced value of off-specification materials: (1) the decrease value of above-baseline feedstocks due to mass displacement by ash; 'offspecification doc', (2) the cost of additional ash disposal; 'disposal doc', (3) the cost associated with sourcing additional feedstock; 'replacement doc', (4) the added cost of processing more material on manufacturing expenses; 'manufacturing doc', and (5) the cost of capital expansion from handling addition quantities of material; 'capital dock'.

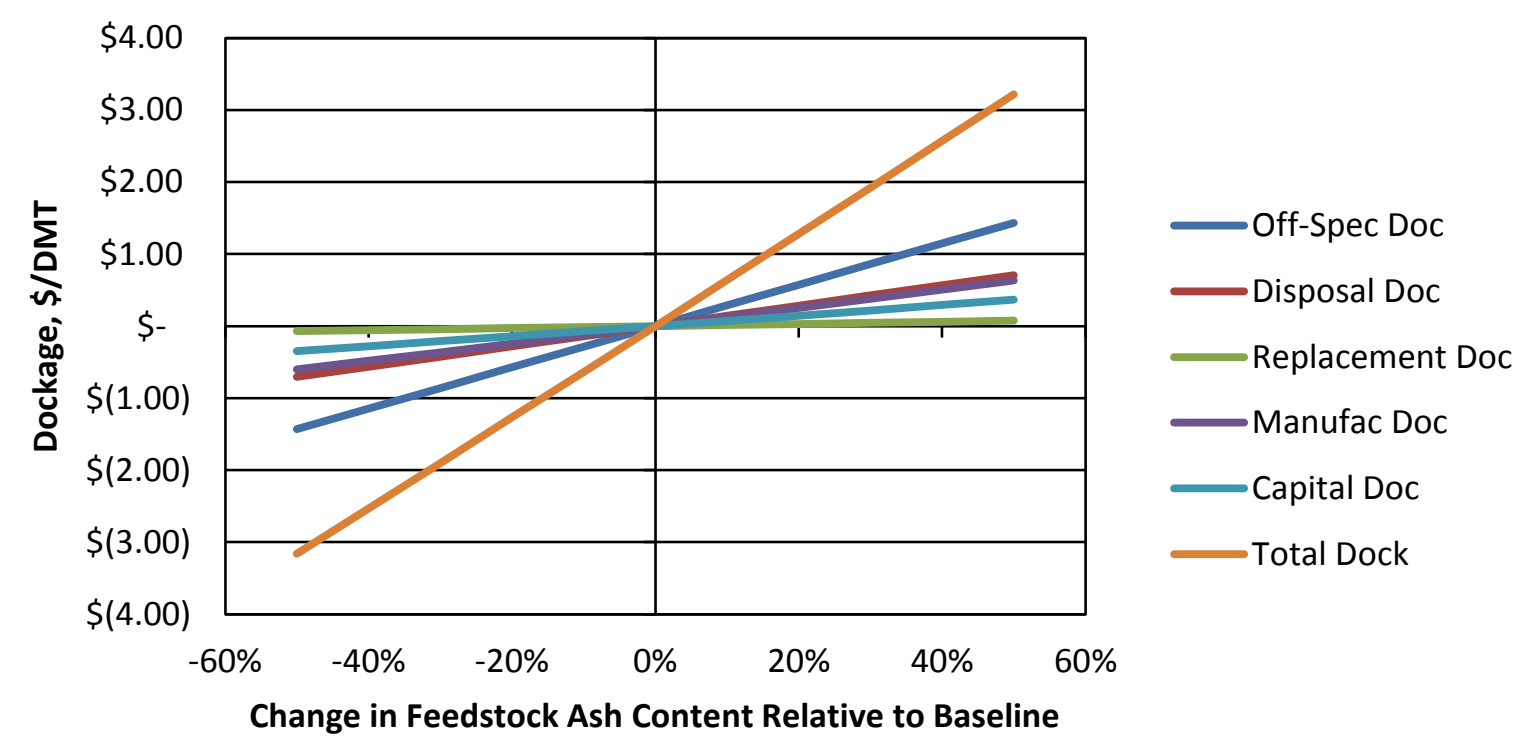

Figure C-1. Sensitivity of dockage by altering feedstock ash content relative to the baseline ash content. of $4.9 \%{ }^{83}$. 
The off-specification dockage was found to be the greatest contributor to the total dockage (45\% of the total dockage), and the most sensitive to increases in delivered feedstock ash content followed by additional disposal (22\% of the total cost; Figure C-1, where a $50 \%$ relative increase in ash equates to $7.4 \%$ ash). This is important as the dockage associated with delivering offspecification material, accounting for additional disposal, and the cost of replacement are largely process independent and likely to be accounted for in the biomass supply and logistics cost basis. On the other hand, the estimates of manufacturing and capital are highly process dependent and thus subject to criticism for their inclusion in a feedstock delivery dockage. Furthermore, the argument can be made from this data that if a dockage is to be applied to material arriving above the baseline specification due to its decreased value, materials of value greater (i.e., ash content lower than the baseline) would in theory warrant a positive purchase price incentive. In the case of this data, a feedstock delivered at $-50 \%$ relative ash content to the baseline $(2.5 \%$ ash $)$ would in essence qualify for a $3.16 \$ /$ dry $\mathrm{T}$ bonus on top of the baseline feedstock purchase price of $58.50 \$$ dry $\mathrm{T}$. While this type of system has not yet been proposed for a commercial system, this type of financial motivation may provide further incentive for farmers to invest in single-pass technology as the material generated is truly of higher value to a conversion facility.

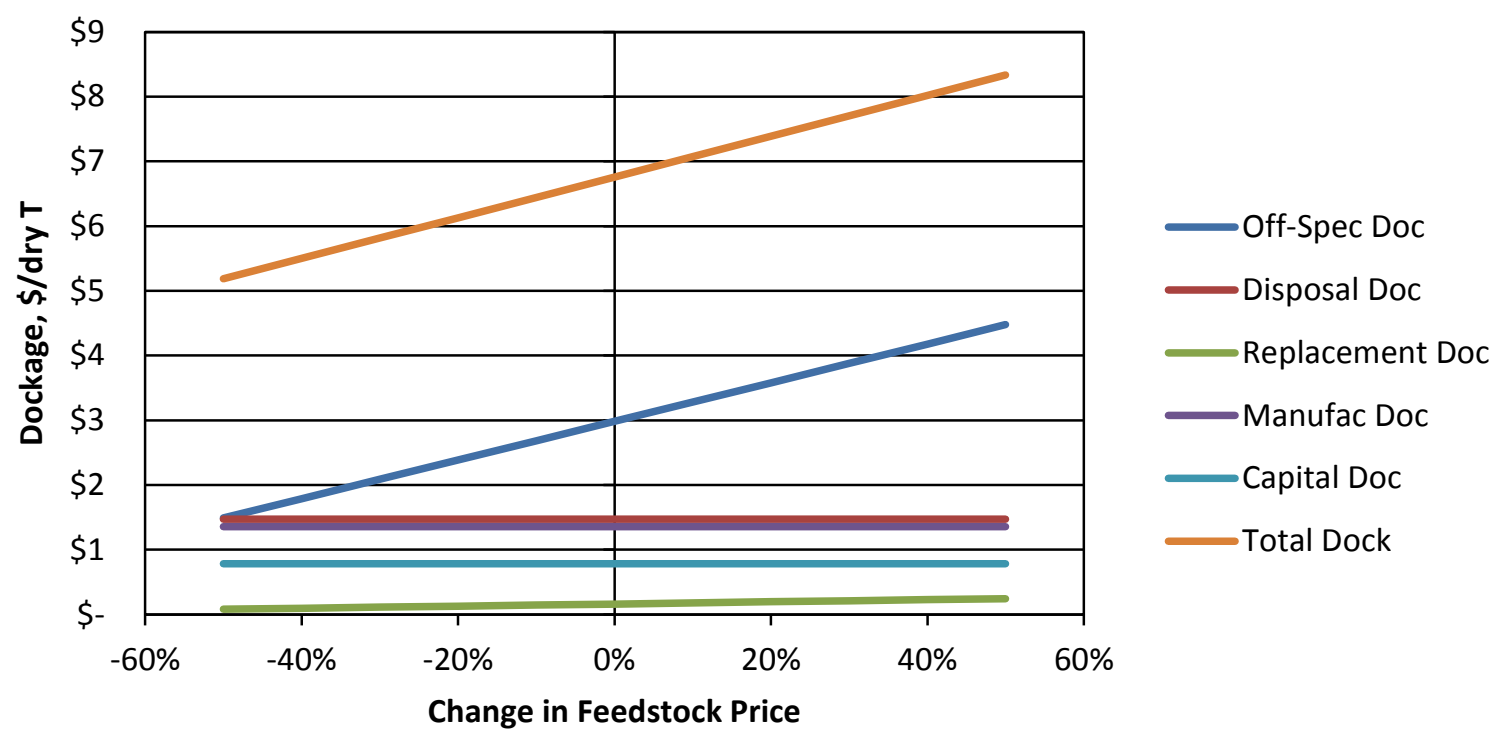

Figure C-2. Sensitivity of the costs impacts to altering feedstock price relative to the base case of 58.50 $\$ /$ dry $\mathrm{T}$ for a delivered feedstock with $10 \%$ ash ${ }^{83}$.

To demonstrate the importance of a proper feedstock procurement cost, the analysis was conducted over a range of feedstock prices, from $29.25 \$$ dry $\mathrm{T}$ to $87.75 \$ /$ dry $\mathrm{T}$ ( $-50 \%$ to $50 \%$ change, respectively) for a delivered feedstock with $10 \%$ ash (Figure C-2). The results clearly show the impact of feedstock price on off-specification cost impacts and ultimately total costs, increasing the total costs by $0.03 \$ / \%$ relative change. When considering the 2017 Design Case target feedstock price of $80 \$ /$ dry T (a $37 \%$ ), the total costs impact rises to $7.91 \$ /$ dry $\mathrm{T}$ for a $10 \%$ ash delivered material, where the off-specification costs now accounts for $52 \%$ of the total cost impacts. This case exemplifies the ability of feedstock price to inflate off-specification cost impacts and potentially lead to a poor estimation of total cost impacts if the other costs associated with high ash are not appropriately modified as well. 


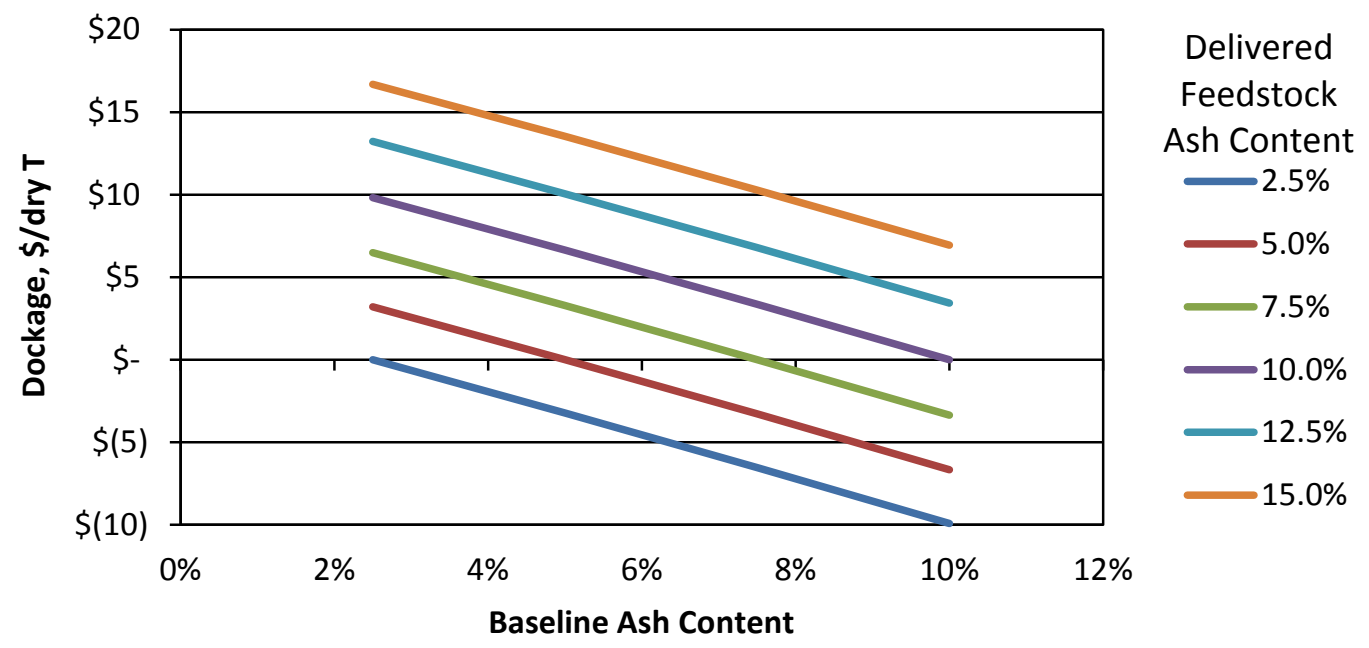

Figure C-3. Total dockage based on shifting baseline ash content for a range of received materials at a feedstock price of $58.50 \$ /$ dry $\mathrm{T}^{83}$.

Finally, the analysis can be used to demonstrate the necessity of defining an appropriate baseline specification for ash content (Figure C-3). Intuitively, as a baseline specification for ash content is raised, the dockage for above-spec material decreases. Figure $\mathrm{C}-3$ shows this rate of change at $1.30 \$ / \%$ ash change for the highest ash material (15\%) and increases to $1.32 \$ / \%$ ash change for the lowest ash material $(2.5 \%)$. This data stresses the importance of accurately determining the ash content at which the end user's process truly begins to incur additional operating costs. In the case of these examples, increasing the baseline ash content to $7.5 \%$ would reduce the dockage of a $10 \%$ ash delivered material by $50 \%$. While it remains to be seen if the costs on material conversion are as sensitive to ash content as the current baseline suggests, this analysis shows the large financial implications to feedstock suppliers when material is compared against a strict specification.

These analyses clearly show the negative implications of off-spec ash content in the baseline conversion system chosen. That said, the variability in dockage with respect to the magnitude of contamination, the price of feedstock, and the baseline ash content highlights the importance of properly defining and assessing a particular modeled feedstock logistics and conversion system. Alternatively, because of the large variability in dockage, it is reasonable to suggest that raw feedstocks purchased by a conversion facility need only be assessed in terms of ash content and biomass content (i.e., 'material other than ash') for preliminary determination of a feedstock's value. More rigorous analysis of what the 'material other than ash' consists of is much less variable than the ash content, as discussed previously in this report, decreasing its relative importance in determining dockage and feedstock value. 


\section{Appendix D \\ Chemical Preconversion Design Basis \\ Leaching Technologies for Soluble Ash}

Alkali metals can be removed easily from biomass after grinding with simple water leaching. Alkaline earth metals can be effectively leached with the addition of acid and heat. Simple leaching with water can be accomplished by spraying while in-field (after windrowing), with detraction of the potential for high losses of convertible sugars. Engineered systems for leaching are typically simple in design, allowing for solvent (water or dilute catalyst solutions) addition, collection, and recycle (if necessary); leachate neutralization and treatment (or disposal); and drying. A simple design for leaching in a depot is a drain and fill leaching system ${ }^{84}$. In this design, chopped or ground biomass is conveyed into a leach tank and leach solution (with or without catalyst) is added to achieve the desired percentage solids. Because leaching is solubility limited, lower percentage solids are preferred; however, if more than one leach cycle can be accommodated, then higher percentage solids could be used, thereby reducing water usage. After leaching, the leach solution is drained to a waste tank, and fresh water is introduced to wash the remaining soluble ash from the biomass (this may occur in several cycles as well). The wash liquid is drained to the waste tank as well. Once washing is completed, the solids are conveyed from the leach tank through a roller press, which mechanically dewaters the biomass. The solids are conveyed to a wet mill and then to a wet pelleting mill. The liquids in the waste tank are neutralized and processed by reverse osmosis, from which the permeate is recycled to the makeup water and the retentate is pumped to wastewater treatment. These operations and the accompanying assumptions are summarized in Table B-2. 
Table D-1. Expected unit operations and assumptions for the application of a drain and fill leaching system for the removal of soluble ash from biomass in a feedstock depot.

\section{Expected Unit Operation}

Convey chopped herbaceous biomass or shredded MSW to leach tank

Leaching (water or dilute acid, steam heat with agitation)
Assumptions

- Herbaceous: 2-in. screen

- MSW: 6-in. screen

- Volume tied to depot throughput

- Fill and drain leach tank with agitation

- $10 \mathrm{wt} \%$ solids

- 5 cycles of 1 hour

- $\quad 95 \%$ reduction of alkali metals and alkaline earth metals

- Sulfuric acid at $0.5 \mathrm{wt} \%$

- Heated to $40^{\circ} \mathrm{C}$

- Pump not needed

- Pump not needed

- 5 cycles of 30 minutes

- Exiting solids are $50 \%$ moisture

- Greater than $95 \%$ recovery of solids

- Exiting solids are $30 \%$ moisture

- Particle size $6 \mathrm{~mm}$ or less for herbaceous and MSW

Convey wet solids to wet mill

Convey wet solids to wet pelletization

Neutralize liquids in waste tank

Pump neutralized liquids to reverse osmosis unit and recycle permeate to makeup water

Pump retentate to wastewater treatment
NA

- $\quad$ Final pH 6-8

- Bicarbonate used for acid

- $90 \%$ removal of ions

- $90 \%$ recovery of permeate water

- $\quad$ Flux $=40 \mathrm{~L} \mathrm{~m}^{-2} \mathrm{~h}^{-1}$

\section{Ash Removal Technologies for Non-Leachable Ash Components}

Silica typically is the largest ash component in biomass and is insoluble in acid and water (it cannot be leached). Hence, physical and/or chemical methods are required to remove silica from biomass with minimal loss of organic material. Technologies that can potentially accomplish this goal include grinding to micron-size particles followed by triboelectrostatic separation and alkali-based processes that dissolve silica ${ }^{45}$. Methods for lignin recovery and precipitation must be employed in the latter case to avoid significant losses of organic material. These methods would add unit operations to the feedstock supply chain that exists today, thereby increasing costs.

Increased costs would arise in the triboelectrostatic separation pathway through increased grinding cost and the requirement that ground biomass be completely dry, as well as through losses of some of the convertible matter with the silica. If considered as an addition to the existing feedstock supply unit operations, the silica dissolution method would increase costs through the requirement for alkali recovery and the requirement for lignin recovery via acid precipitation, ultrafiltration, or triboelectrostatic separation from the lignin after concentration and drying. Acid-soluble lignin also could be lost. However, it is notable that the severity of alkaline treatment required to solubilize the silica would likely disrupt the structure of 
herbaceous biomass sufficiently to greatly reduce grinding and pelletization energy requirements. In any event, if silica or heteroatoms must be removed for a given conversion process, it may be more cost effective to design the feedstock supply system around the removal processes rather than vice versa.

\section{Cost Estimation for Chemical Preconversion}

Few economic analyses are available in the literature for ash removal. The following provides overall costs for two systems that are found in the literature and include a technoeconomic analysis of leaching of alkali metals from rice straw for combustion and a technoeconomic analysis of removing both alkali metals and silica from non-woody residues (also for combustion). The cost of $95 \%$ alkali metal removal from rice straw by leaching was estimated in 2000 to be $\$ 13.61$ to $\$ 16.33 /$ dry T (Bakker 2000). A more recent analysis for non-woody feedstocks estimated a net cost of $\$ 39.93$ to $\$ 60.80 /$ dry T for removal of alkali metals (up to $95 \%$ ) by leaching, followed by removal of silica (up to $75 \%$ ) with triboelectrostatic separation ${ }^{45}$.

Utilizing mechanical and chemical ash removal technologies in tandem to reduce the amount of non-specification feedstock blend components requiring further preprocessing to meet ash specifications is a strategy for reducing ash while still meeting cost targets. This can be accomplished by utilizing fractional grinding to take advantage of the skewed distribution of ash toward smaller particle sizes for corn stover. Therefore, the performance target for chemical preconversion is to produce on-spec feedstocks below the biorefinery's added costs for offspecification feedstock by either reducing the amount of feedstock requiring chemical ash removal, reducing the cost of chemical ash removal, or both. 


\section{Appendix E \\ Cost Calculation}

The economic analysis was designed for an 800,000 ton/year pellet demand to produce hydrocarbon fuels.

About cost year indices: The cost-year of 2011 was chosen for this analysis to keep the consistency across all DOE-BETO platforms for which similar "design case target" reports are being established during 2013-2014 efforts. Capital costs provided in a year other than 2011\$ were adjusted using the Plant Cost Index from Chemical Engineering Magazine to a common basis year of 2011 .

The general formula for year-dollar back-casting is:

$$
2011 \text { Cost }=(\text { Base Cost })\left(\frac{2011 \text { Cost Index }}{\text { Base Year Index }}\right)
$$

Ownership cost: Ownership cost consists of interests and depreciation cost, and insurance, housing and taxes cost and owning the facility.

\section{Interest and depreciation}

The ASABE lists two different methods for costing depreciation and interest: (1) calculate depreciation and interest separately, or (2) calculate depreciation and interest on the value to be depreciated and then calculate interest on the salvage value. The AAEA uses the second method, which can be expressed as Equation (1):

$$
I \& D=(P-S)\left[\frac{(i)(1+i)^{n}}{\left\{(1+i)^{n}\right\}-1}\right]+S \times i
$$

where

I \& D = Interest and Depreciation

$\mathrm{P} \quad=$ purchase price of equipment

$\mathrm{i}=$ annual interest rate

$\mathrm{n}=$ life of the equipment in years

$\mathrm{k}=$ sum of rates for taxes, housing (shelter), insurance

$\mathrm{S}=$ salvage value (salvage value $\% \times$ list price) (salvage value percent assumed in the present study is $30 \%$ )

Salvage value (remaining value) must be known or estimated to determine interest and depreciation. The America Society of Agricultural and Biological Engineers ${ }^{85}$ (ASABE) method was used for determination of salvage value (ASABE, D496.31, 2006, Section 6.2.2).

Insurance, Housing, and Taxes (IH\&T)

Insurance, housing (cost of shelter for equipment), and taxes (IH\&T) refer to the fixed costs related to the equipment, and these costs are estimated as percentages of the purchase price 
(Equation 2). If actual data are not available, the ASABE suggests using the following percentages: taxes $1.00 \%$, housing $0.75 \%$, and insurance $0.25 \%$, for a total of $2.00 \%$.

$$
I H \& T=\frac{\left(I_{\text {Percentage }}+H_{\text {Percentage }}+T_{\text {Percentage }}\right) \times \text { average }(\text { purchase price, salvage })}{\text { Work hours per year } \times \text { eff ciency factor }}=\frac{\$}{h r}
$$

Operating cost: Operating cost consists of repair \& maintenance, fuel and labor cost. Expenditures are necessary to keep a machine operable due to wear, part failure, accidents, and natural deterioration. The costs for repairing a machine are highly variable. Good management may keep costs low. In the present study, the following equation is used for calculating the repair and maintenance $(\mathrm{R} \& \mathrm{M})$ costs.

$$
R \& M=\frac{\text { list price } \times \text { repairs and maintenance percentage }}{\text { life time }(\mathrm{hr})}=\frac{\$}{\mathrm{hr}}
$$

Repairs and maintenance percentage is estimated based on percentage of machine price. Fuel consumption cost is calculated based on actual $\mathrm{kW}$ data obtained either from machinery specifications or from actual estimates obtained from laboratory-scale and pilot-scale experimental data. Labor rates were obtained from the Idaho Bureau of Labor Statistics, and labor hours were based on assumed shift schedules. The total working hours include three shifts, 40 hours/week, and 50 weeks per year. The assumed labor rate for horizontal bale grinder, hammer mill, dryer, pallet mill and chemical pretreatment are $\$ 15.88, \$ 19.88, \$ 15.51, \$ 15.51$ and $\$ 19.88$ respectively. Also, we have assumed that one person will be able to manage two machines. 


\section{Future Work}

Foremost, efforts must build upon collaborative interactions with the biochemical and thermochemical conversion platforms to gain a better understanding of feedstock quality and conversion performance. Efforts must also focus on robust screening techniques and methodologies to verify feedstock quality. Relative to ash determinations, a bulk biomass screening tool that is easy to use, rugged, has low maintenance requirements and field-applicable is needed to better understand feedstock variability (temporal, seasonal), logistic and preprocessing intermediates changes and variability and options for mitigating impacts; bringing to bear all the "architectural' requirement to support the biorefinery quality specification, and the initial and intermediate specifications that sustain those specifications. 